\title{
Seismic Anisotropy at the Rotokawa and Ngatamariki Geothermal Fields in the Taupo Volcanic Zone
}

\author{
by \\ Stefan Mroczek
}

A thesis submitted to Victoria University of Wellington in partial fulfilment of the requirements for the degree of

Master of Science in Geophysics

School of Geography, Environment and Earth Sciences

Victoria University of Wellington

Wellington, New Zealand

2017 



\section{Abstract}

In order to investigate the cracks/fractures in the geothermal fields of Rotokawa and Ngatamariki, we measure seismic anisotropy across both fields and interpret the results in the context of stress aligned microcracks. Cracks aligned perpendicular to the direction of maximum horizontal stress close and their fluid is forced into cracks aligned with maximum horizontal stress $\left(S_{H_{\max }}\right)$. Seismic anisotropy is the directional dependence of a seismic wave's velocity and provides a measure of crack orientation and density.

To measure seismic anisotropy we conduct shear wave splitting measurements on 52,000 station-earthquake pairs across both Rotokawa and Ngatamariki from earthquakes recorded during 2015. Both fields are the subject of other geophysical and geological studies. Thus they are excellent subjects for studying seismic anisotropy.

We cluster our measurements by their station-event path and fit the parameters from these clusters to those from theoretical crack planes. We also apply 2-D tomography to shear wave splitting time delays $(\delta t)$ and spatial averaging to shear wave splitting fast polarisations $(\phi)$. In addition, we compare time delays with $\mathrm{P}$-wave to S-wave velocity ratios $\left(v_{P} / v_{S}\right)$.

Local measurements of stress within Rotokawa and regional measures of stress within the Taupo Volcanic Zone provide a comparison for the shear wave splitting measurements. We measure $\phi$ which agrees with the NE-SW regional direction of $S_{H_{\max }}$ across Ngatamariki and parts of Rotokawa. Within Rotokawa, we observe a rotation of $\phi$ away from NE-SW toward N-S that agrees with borehole measurements of direction of $S_{H_{\max }}$ of $023^{\circ}$ and $030^{\circ}$. Spatial averaging of $\phi$ reveals mean orientations close to the strike of nearby active faults.

The theoretical crack planes, that fit best to the shear wave splitting measurements, correspond to aligned cracks striking $045^{\circ}$ outside of both fields, $035^{\circ}$ within Ngatamariki, and $035^{\circ}$ through to $0^{\circ}$ within Rotokawa. 
The average percent anisotropy for the full dataset, approximately $4 \%$, is close to the upper bound for an intact rock. Delay time tomography shows regions of higher delay time per kilometre of path length $(\mathrm{s} / \mathrm{km})$ within both fields and possibly associated with the production field fault in Rotokawa.

$v_{P} / v_{S}$ shows a wide range of normally distributed values, from 1.1 through to 2.4 with a mean of 1.6, indicating a mixture of gas filled and saturated cracks. A positive correlation between delay time per kilometre $(\delta t p k m)$ and $v_{P} / v_{S}$ indicates that the majority of the cracks are saturated. 


\section{Acknowledgements}

I would like to sincerely thank my supervisor Martha Savage for her all her support. Her advice, insight, and feedback have been invaluable in conducting this research.

I would also like to thank Mercury and the Tauhara No.2 Trust for generously funding this research through awarding me a scholarship and providing access to relevant data for this project. Also my thanks to those who provided a point of contact at Mercury; Simon Addison, Ian Richardson, and Paul Siratovich.

I would also like to acknowledge those whose time and help made this project possible: Chet Hopp for providing matched filter detections and producing all the waveforms I needed. Hubert Zal for his expertise in orientating borehole stations. Steven Sewell for his feedback and help from his knowledge of Rotokawa and Ngatamariki. David McNamara for kindly providing in-situ stress measurements. Aleksandr Beliaev for his help with Linux. Richard Arnold for his introduction into the world of circular statistics and feedback on my statistics writing. Also my father, Ed Mroczek, for his continued feedback and support.

And finally, for discussions and Friday drinks: My office mates; Hamish Hirschberg, Andy McNab, and Nicolas Oestreicher. And other fellow students.

The R statistical programming language (R Core Team 2013) is used throughout this thesis with a number of additional packages for plotting and analysis (Appendix E.1). Map tiles are by Stamen Design, under CC BY 3.0. Map data is by OpenStreetMap, under ODbL. 


\section{Contents}

$\begin{array}{ll}\text { Abstract } & \text { i }\end{array}$

Acknowledgements

Contents $\quad$ v

List of Figures $\quad$ xi

List of Tables $\quad$ xvii

1 Introduction 1

1.1 Motivation . . . . . . . . . . . . . . . . . . . 1

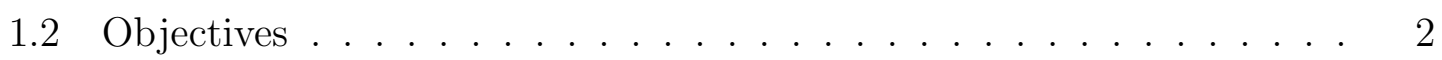

1.3 Geological and geophysical setting . . . . . . . . . . . . . 3

1.3.1 Taupo Volcanic Zone . . . . . . . . . . . . . . . . . . 3

1.4 Previous studies . . . . . . . . . . . . . . 6

1.4.1 Shear wave anisotropy studies . . . . . . . . . . . . . 6

1.4 .2 Stress studies ..................... 8

1.5 Earthquake catalogue . . . . . . . . . . . . . . . . 10

1.5.1 Seismometer network . . . . . . . . . . . . . . 10

1.5.2 Location . . . . . . . . . . . . . . . . 11

1.5.3 Matched filter detection . . . . . . . . . . . . 13

2 Seismic anisotropy \& shear wave splitting $\quad 15$

2.1 Overview . . . . . . . . . . . . . . . . . . . . 15 
2.2 Theoretical background . . . . . . . . . . . . . . . . 16

2.2.1 Hexagonal Symmetry . . . . . . . . . . . . . . . . . . 16

2.2 .2 Velocity in an anisotropic material . . . . . . . . . 16

2.3 Fluid-filled cracks \& shear wave splitting . . . . . . . . . . . . 21

2.3.1 Stress aligned microcracks . . . . . . . . . . . . . . 21

2.3.2 A note on terminology . . . . . . . . . . . 22

2.3.3 Faults versus cracks . . . . . . . . . . . 22

2.3 .4 Crack scale . . . . . . . . . . . . . . . . . 24

2.3.5 Evidence for fluid-filled cracks . . . . . . . . . . . . . 24

2.4 Further anisotropic considerations . . . . . . . . . . . . 25

2.4.1 Propagation angle . . . . . . . . . . . . . 25

$2.4 .290^{\circ}$ flips . . . . . . . . . . . . . . . . 25

3 Methodology $\quad 27$

3.1 Methods Summary . . . . . . . . . . . . . . . . 27

3.2 Statistical tests and methods . . . . . . . . . . . . . . . . . . 29

3.2 .1 Circular statistics . . . . . . . . . . . . . . . . 29

3.2.2 The von Mises distribution . . . . . . . . . . . . . . . 31

3.2 .3 Rayleigh test . . . . . . . . . . . . . . . . . . 31

3.2.4 Watson's non-parametric test ............. 33

3.2.5 Pearson's product-moment correlation . . . . . . . . . . . 34

3.2 .6 Bootstrapping . . . . . . . . . . . . . . . 34

3.2.7 Standard error . . . . . . . . . . . . . . 35

3.2 .8 Hierarchical clustering . . . . . . . . . . . . . . . . . 35

3.3 Station orientation . . . . . . . . . . . . . 36

3.3.1 Rayleigh-Wave method . . . . . . . . . . . . . . 37

3.3.2 Processing . . . . . . . . . . . . . . . . . . . . . . . 38

3.4 Automatic shear wave picking . . . . . . . . . . . . . . . 43 
3.4.1 S-picker overview . . . . . . . . . . . . . . . . . . 43

3.4.2 S-picker methodology . . . . . . . . . . . . . . . . . . 43

3.4.3 spickerC modifications . . . . . . . . . . . . . . 45

3.4 .4 Grading ..................... 46

3.4.5 Processing parameters . . . . . . . . . . . . . 46

3.5 Automatic shear wave splitting (MFAST) . . . . . . . . . . . 49

3.5.1 Filtering . . . . . . . . . . . . . . . 49

3.5.2 Measuring technique . . . . . . . . . . . . . . 50

3.6 Cycle skipping . . . . . . . . . . . . . . . . . 54

3.6 .1 Grading ....................... 55

3.6 .2 Incoming angle . . . . . . . . . . . . . . . . . . 57

3.7 Earthquake path clustering . . . . . . . . . . . . . 59

3.7 .1 movMF . . . . . . . . . . . . . . . . 59

3.7.2 Processing . . . . . . . . . . . . . . . . 60

3.8 Crack fitting with MSAT . . . . . . . . . . . . . . 62

3.8.1 Crack model . . . . . . . . . . . . . . . . . 62

3.8 .2 Fitting process . . . . . . . . . . . . 63

3.8.3 Angle of incidence versus angle of propagation . . . . . . . . 67

3.8.4 Applications of restricting measurements . . . . . . . . . . . 69

3.9 Tomography estimation and spatial averaging (TESSA) . . . . . . 70

3.9 .1 Dataset restriction . . . . . . . . . . . . 71

3.9 .2 Quadtree gridding . . . . . . . . . . . . . . 72

3.9.3 Tomography estimation . . . . . . . . . . . 73

3.9.4 Spatial averaging ................... 73

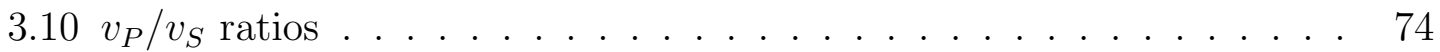

4 Results and discussion $\quad 75$

4.1 Station orientation . . . . . . . . . . . . . . . . 75 
4.1.1 Errors in orientation . . . . . . . . . . . . 76

4.2 Shear wave picking $($ spickerC $) \ldots \ldots \ldots$. . . . . . . . 80

4.2.1 Errors in S-wave picks . . . . . . . . . . . . . . . 80

4.3 Shear wave splitting (stations) . . . . . . . . . . . . . 82

4.3.1 Assumptions. . . . . . . . . . . . . . . . . 82

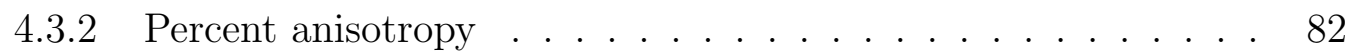

4.3.3 Matched filter detections versus templates . . . . . . . . 87

4.3.4 Rayleigh test failures . . . . . . . . . . . . . . . 88

4.3.5 Rotokawa in-situ cracks . . . . . . . . . . . . . 90

4.4 Crack fitting . . . . . . . . . . . . . . . . . . . . . . . 91

4.4 .1 Summary ...................... 92

4.4.2 Fitting with uniform velocity model . . . . . . . . . . 110

4.5 TESSA . . . . . . . . . . . . . . . . 117

4.5.1 Full dataset . . . . . . . . . . . . . . . . . . . . 118

4.5.2 Restricted dataset . . . . . . . . . . . . . . . 121

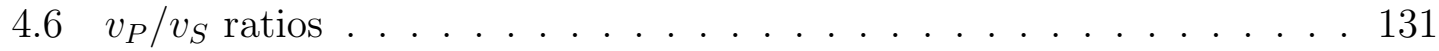

$4.6 .1 \quad v_{P} / v_{S}$ and delay time . . . . . . . . . . . . 131

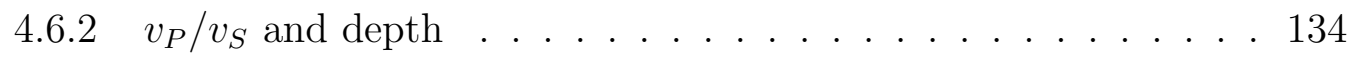

5 Further discussion $\quad 137$

5.1 The shear wave window . . . . . . . . . . . . . . 137

5.2 Borehole versus surface stations . . . . . . . . . . . . . . . . . . 140

5.3 Grades . . . . . . . . . . . . . . . . . . . . . . 141

5.4 Variations through time . . . . . . . . . . . . . . . . . . 144

5.5 Measurement rates . . . . . . . . . . . . . . . . . . . . . . 144

5.6 Theoretical distribution of polarisations . . . . . . . . . . . . . 145

5.6.1 Accounting for bimodality . . . . . . . . . . . . . 147

5.7 Applications . . . . . . . . . . . . . . . . 154 
5.8 Future work . . . . . . . . . . . . . . . . . . . . . . . . . 154

$\begin{array}{lll}6 & \text { Conclusions } & 157\end{array}$

6.1 Fitting of crack planes . . . . . . . . . . . . . . . . 158

6.2 Spatial averaging . . . . . . . . . . . . . . . . . . . 158

6.3 Delay time tomography . . . . . . . . . . . . . . . . . . . . 158

$6.4 v_{P} / v_{S}$ and cracks . . . . . . . . . . . . . . . 159

6.5 Restricting the dataset . . . . . . . . . . . . . . . . . 159

6.6 Synopsis . . . . . . . . . . . . . . . . . . . . 160

$\begin{array}{ll}\text { Bibliography } & 161\end{array}$

$\begin{array}{ll}\text { Appendix A Tables } & 173\end{array}$

$\begin{array}{ll}\text { Appendix B Fitted crack planes } & 179\end{array}$

$\begin{array}{ll}\text { Appendix C Parameters } & 189\end{array}$

C.1 spickerC . . . . . . . . . . . . . . . . . . . . . . . . . . . 189

C.2 MFAST . . . . . . . . . . . . . . . . . 191

$\begin{array}{ll}\text { Appendix D MSAT functions } & 195\end{array}$

D.1 MS_hudson_cracks . . . . . . . . . . . . . . . . . . . . . . 195

D.2 MS_rot3 . . . . . . . . . . . . . . . . . . . . . . . 197

D.3 MS_phasevels . . . . . . . . . . . . . . . . . . . . . . . . 199

$\begin{array}{lll}\text { Appendix E R tools for MFAST } & 201\end{array}$

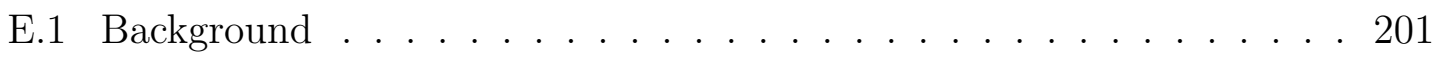

E.1.1 Required packages . . . . . . . . . . . . . . 202

E.2 Functions . . . . . . . . . . . . . . . . . . . . . . 202

E.2.1 sm.createTESSA _. . . . . . . . . . . . . . 202

E.2.2 sm.getevents. . . . . . . . . . . . . . . . 203

E.2.3 sm.getevents . . . . . . . . . . . . . . . . . 204

E.2.4 sm.pathclus . . . . . . . . . . . . . . . 205 


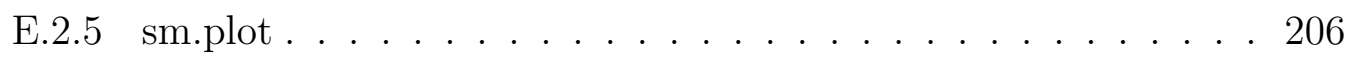

E.2.6 sm.proj . . . . . . . . . . . . . . . 207

E.2.7 sm.read . . . . . . . . . . . . . . . . 209

E.2.8 sm.readraw . . . . . . . . . . . . . . . . . 211

E.2.9 sm.stde . . . . . . . . . . . . . . . . . . 212

$\begin{array}{lll}\text { Appendix F NZGW conference paper } & \mathbf{2 1 5}\end{array}$ 


\section{List of Figures}

1.1 Geothermal field locations with respect to the North Island of New Zealand. . . . . . . . . . . . . . . . . . . 4

1.2 Rotokawa and Ngatamariki fields outlined with resistivity boundaries 5

1.3 Rotokawa in-situ stress orientations (McNamara et al. 2015) . . . . . 9

1.4 Histogram of earthquake depths . . . . . . . . . . . . . . . . . . . 12

2.1 Pole figure of anisotropy parameters for vertical cracks striking $045^{\circ}$. 21

2.2 Diagram of shear wave splitting polarisation and delay time arising from stress aligned cracks . . . . . . . . . . . . . . 23

3.1 Example of the density of the von Mises Distribution . . . . . . . . 32

3.2 Example of a "bad" station orientation estimate from the RayleighWave method . . . . . . . . . . . . . . . . . . . 40 40

3.3 Example of a "questionable" station orientation estimate from the Rayleigh-Wave method . . . . . . . . . . . . . . . . . . . . . 41

3.4 Example of a "good" station orientation estimate from the RayleighWave method . . . . . . . . . . . . . . . . . . 42

3.5 Histogram of the time difference between manual and automatic Swave picks for the 2013 training events . . . . . . . . . . . . . . . 48

3.6 Contours of the smallest eigenvalue of the covariance matrix for a high grade shear wave splitting measurement . . . . . . . . . . 52 
3.7 Example of linearisation of the particle motion by the best inverse splitting operator ...................... 53

3.8 Clustering of shear wave splitting measurements from multiple time windows . . . . . . . . . . . . . . . . . . . 54

3.9 Contours of the smallest eigenvalue of the covariance matrix for a cycle skipped shear wave splitting measurement . . . . . . . . . . . . 56

3.10 Pierce points of station-event paths clustered on a unit hemisphere (Station RT01) . . . . . . . . . . . . . . . 61

3.11 Pole figure showing the anisotropy parameters for the best fitting crack plane for shear wave splitting measurements from station RT05 . . . 65

3.12 Example of misfit grids of shear wave splitting measurements fit to theoretical crack planes . . . . . . . . . . . . . . . . . 68

3.13 Example of a lower hemisphere projection (of anisotropy parameters of the best fitting crack plane) overlaid with real and modelled shear wave splitting polarisations . . . . . . . . . . . . . . 70

4.1 Summary of orientation results for Station NS12 . . . . . . . . . . 77

4.2 Summary of orientation results for Station NS13 . . . . . . . . . . 78

4.3 Summary of orientation results for Station NS14 . . . . . . . . . . . 79

4.4 Histogram of S-wave pick time uncertainty . . . . . . . . . . . . . . 81

4.5 Rose diagrams of shear wave splitting fast polarisations for each station 85

$4.6 \%$ anisotropy versus station-event path distance . . . . . . . . . . 87

4.7 Shear wave splitting parameters of matched filter detected events versus template events . . . . . . . . . . . . . . . . . . . . . 89

4.8 Rotokawa in-situ stress orientations compared with fast polarisations from Group 30 . . . . . . . . . . . . . . . . . . . . . . 92

4.9 Lower hemisphere projections of anisotropy parameters plotted over spatial location . . . . . . . . . . . . . . . . . 94 
4.10 Summary of Group 11 shear wave splitting measurements (pole plot, misfit grid, and ray paths $\ldots \ldots \ldots$. . . . . . . . . . . . 96

4.11 Comparison between angle preserving, spherical, and equal area pro-

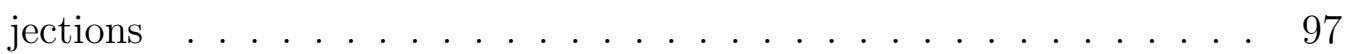

4.12 Summary of Group 25 shear wave splitting measurements (pole plot, misfit grid, and ray paths $\ldots \ldots \ldots$. . . . . . . . . . . . . . 98

4.13 Summary of Group 22 shear wave splitting measurements (pole plot, misfit grid, and ray paths $\ldots \ldots$. . . . . . . . . . . . . . 99

4.14 Summary of Group 30 shear wave splitting measurements (pole plot, misfit grid, and ray paths) . . . . . . . . . . . . 100

4.15 Summary of Group 7 shear wave splitting measurements (pole plot, misfit grid, and ray paths) . . . . . . . . . . . . . 101

4.16 Summary of Group 38 shear wave splitting measurements (pole plot, misfit grid, and ray paths) . . . . . . . . . . . . . . . 102

4.17 Spherical projection of Group 25's best fitting crack plane . . . . . . 103

4.18 Summary of Group 8 shear wave splitting measurements (pole plot, misfit grid, and ray paths) . . . . . . . . . . . . . 104

4.19 Summary of Group 18 shear wave splitting measurements (pole plot, misfit grid, and ray paths) . . . . . . . . . . . . . 105

4.20 Summary of Group 14 shear wave splitting measurements (pole plot, misfit grid, and ray paths $\ldots \ldots \ldots 6$

4.21 Summary of Group 34 shear wave splitting measurements (pole plot, misfit grid, and ray paths $\ldots \ldots$. . . . . . . . . . . 107

4.22 Summary of Group 28 shear wave splitting measurements (pole plot, misfit grid, and ray paths) . . . . . . . . . . . . . . 111

4.23 Summary of Group 43 shear wave splitting measurements (pole plot, misfit grid, and ray paths) . . . . . . . . . . . . . . . 112 
4.24 Summary of Group 40 shear wave splitting measurements (pole plot, misfit grid, and ray paths) . . . . . . . . . . . . . . 113

4.25 Group 39 (straight line) shear wave splitting measurements (pole plot, misfit grid, and ray paths) . . . . . . . . . . . . . 115

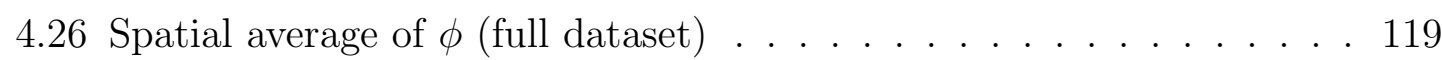

4.27 Spatial average of $\phi$ (Rotokawa) . . . . . . . . . . . . . . 120

4.28 Delay time tomography (full dataset) . . . . . . . . . . . . . . 122

4.29 Full dataset TESSA checkerboard tests . . . . . . . . . . . . . . . . 123

4.30 Full dataset TESSA raypaths and checkerboard test resolution . . . . 124

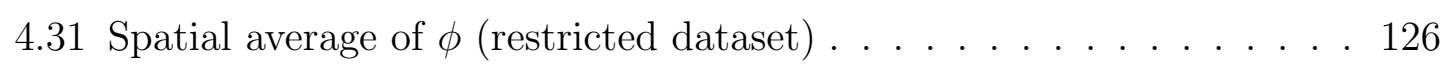

4.32 Delay time tomography (restricted dataset) . . . . . . . . . . 128

4.33 Restricted dataset TESSA raypaths and checkerboard test resolution 129

4.34 Restricted dataset TESSA checkerboard test . . . . . . . . . . . . . 130

4.35 Histogram of $v_{P} / v_{S} \ldots \ldots \ldots \ldots$. . . . . . . . . . . . 132

$4.36 v_{P} / v_{S}$ versus delay time . . . . . . . . . . . . . 133

$4.37 v_{P} / v_{S}$ versus delay time (restricted dataset) $\ldots \ldots \ldots$. . . . . . 135

$4.38 v_{P} / v_{S}$ versus event depth . . . . . . . . . . . . 136

5.1 Clustered station-event path pierce points for station RT01 . . . . . . 139

5.2 Theoretical fast polarisation distribution for randomly sampled propagation angles . . . . . . . . . . . . . . . . . . 148

5.3 Theoretical fast polarisation distribution for randomly sampled propagation angles from a dipping crack plane . . . . . . . . . . . . 149

5.4 Theoretical fast polarisation distribution for randomly sampled propagation angles (bimodal assumption) . . . . . . . . . . 151

5.5 QQ \& PP plots for Jones-Pewsey distribution fit to theoretical fast

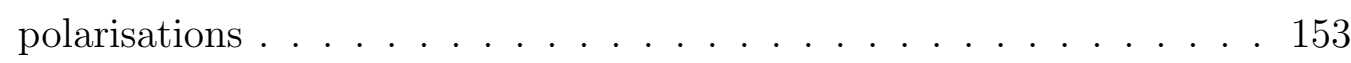


B.1 Summary of Group 26 shear wave splitting measurements (pole plot, misfit grid, and ray paths) . . . . . . . . . . . . . . . 181

B.2 Summary of Group 53 shear wave splitting measurements (pole plot, misfit grid, and ray paths) . . . . . . . . . . . . . . 182

B.3 Summary of Group 37 shear wave splitting measurements (pole plot, misfit grid, and ray paths $\ldots \ldots$. . . . . . . . . . . . 183

B.4 Summary of Group 3 shear wave splitting measurements (pole plot, misfit grid, and ray paths) . . . . . . . . . . . . . 184

B.5 Summary of Group 29 shear wave splitting measurements (pole plot, misfit grid, and ray paths $\ldots \ldots \ldots$. . . . . . . . . . 185

B.6 Summary of Group 31 shear wave splitting measurements (pole plot, misfit grid, and ray paths) . . . . . . . . . . . 186

B.7 Dendrogram of hierarchical clustering for misfit grids . . . . . . . . . 187 


\section{List of Tables}

3.1 Hierarchical clustering demonstration . . . . . . . . . . . . . . 36

3.2 "verylocal" waveform filters for shear wave splitting (MFAST) . . . . 51

3.3 1D velocity model used to determine incidence angles . . . . . . . . 58

3.4 Parameters of the Hudson crack model used for fitting shear wave splitting measurements . . . . . . . . . . . . . . 63

3.5 Processing parameters for delay time tomography and polarisation spatial averaging $(\mathrm{TESSA}) \ldots \ldots \ldots 74$

4.1 Orientations of borehole stations determined from Rayleigh wave polarisations ............................ 76

4.2 Mean shear wave splitting parameters for each station $(\mathrm{p}$-value $\leq 0.05) 83$

4.3 Mean shear wave splitting parameters for each station $(\mathrm{p}$-value $>0.05) 84$

4.4 Mean shear wave splitting parameters for measurements from each fitted crack plane . . . . . . . . . . . . . . . . . . . 93

4.5 Mean shear wave splitting parameters for measurements from each fitted crack plane (straight line) . . . . . . . . . . . . . . 116

5.1 Elevations of borehole stations . . . . . . . . . . . . . . . . . . 141

5.2 Number of measurements at each shear wave splitting grade (F1, F2, F3) at each station . . . . . . . . . . . . . . . . 143

5.3 Mean pick rate and splitting rate for detection and template events . 145 
5.4 Mean shear wave splitting parameters for each station (bimodal, pvalue $\leq 0.05) \ldots \ldots \ldots \ldots \ldots$. . . . . . . . . . . . . 152

5.5 Mean shear wave splitting parameters for each station (bimodal, pvalue $>0.05) \ldots \ldots \ldots \ldots \ldots \ldots \ldots$

A.1 Telesimic events used for station orientation (2014) . . . . . . . . 174

A.2 Telesimic events used for station orientation (2015) . . . . . . . . 175

A.3 Numbers of shear wave splitting measurements for each station . . . . 176

A.4 Mean pick rate and splitting rate for detection and template events for each station . . . . . . . . . . . . . . . . . 177

A.5 Percent anisotropy estimates for each station . . . . . . . . . . 178

B.1 Strike and dip of fitted crack planes . . . . . . . . . . . . . 180

C.1 Shear wave picking processing parameters (spickerC) . . . . . . . 189

C.2 Shear wave splitting processing parameters (MFAST) . . . . . . . 191 


\section{Chapter 1}

\section{Introduction}

\subsection{Motivation}

Geothermal energy is a renewable energy resource on human time scales (Rybach et al. 2000). The New Zealand government has committed to reducing reliance on nonrenewable (fossil fuel) energy sources and an increased employment of geothermal is important to this goal (Mason et al. 2010). This makes optimisation of geothermal energy an area of great interest both economically and environmentally.

Seismic anisotropy in a fractured (geothermal) reservoir gives information about the orientation and density of cracks within the system (Elkibbi et al. 2005). Knowledge about the orientation and density of fractures is, in turn, potentially useful for optimising field operations (e.g. through inclusion in numerical reservoir models) and for targeting production and injection wells. We determine seismic anisotropy using the shear wave splitting method (Chapter 2).

The geothermal fields of Rotokawa and Ngatamariki, within the Taupo Volcanic Zone (TVZ), both have ongoing seismic monitoring and a catalogue of already well located earthquakes. Being the subject of other geological and geophysical studies, they provide an ideal place for seismological investigations into subsurface structure. 
For example; modelling of magnetotelluric data at Rotokawa (Heise et al. 2008), resistivity at Ngatamariki and Rotokawa (Boseley et al. 2010; Risk 2000), stratigraphy and hydrothermal alteration at Ngatamariki (Boseley et al. 2012), reservoir tracer tests at Ngatamariki (Buscarlet et al. 2015), among others. Importantly, Siratovich et al. (2014), McNamara et al. (2015), and Massiot et al. (2015) study the stress and fractures within Rotokawa.

\subsection{Objectives}

The objective of this study is to asses the seismic anisotropy across Ngatamariki and Rotokawa geothermal fields using the shear wave splitting technique and to interpret the results in the context of stress aligned cracks. By comparing the results from the 2015 catalogue to other measurements of stress orientation, the techniques used here can be evaluated for future use with larger earthquakes catalogues.

In addition to the usual exercises of station averages of shear wave splitting parameters and determination of percent anisotropy, these objectives will be obtained by:

- Clustering measurements by their station-event paths,

- Fitting these clusters to theoretical crack planes that give rise to the anisotropy

- Finally, using these crack planes to inform both spatial averaging and delay time tomography of the shear wave splitting measurements 


\subsection{Geological and geophysical setting}

\subsubsection{Taupo Volcanic Zone}

The Taupo Volcanic Zone (TVZ) is located in the back-arc of the Hikurangi subduction zone in New Zealand's North Island (Figure 1.1) and is the southernmost extent of the Tonga-Kermadec arc system (Cole and Lewis 1981). It exhibits high heat flow which is almost entirely facilitated through the movement of hot water and steam (Stern 1987; Wilson et al. 1995).

The Old TVZ, active from 2 Ma up to $0.35 \mathrm{Ma}$, represents the beginning of activity in the zone (Wilson and Rowland 2016; Wilson et al. 1995). Following this is the Young TVZ which has been active from 0.35 Ma to $61 \mathrm{ka}$ (Wilson and Rowland 2016). The current state of the TVZ is known as the Modern TVZ and has been active for the last $61 \mathrm{kyr}$ (Wilson and Rowland 2016). The extents of the Young and Old TVZ are outlined in Figure 1.1. The TVZ is actively rifting (Wallace et al. 2004) at varying rates along its extent.

Both Ngatamariki and Rotokawa fields are located in the southern region of the central TVZ and are two of twenty-three geothermal systems in the TVZ (Chambefort et al. 2016). The fields are owned and operated by Mercury and the Rotokawa Joint Venture (Mercury and Tauhara North No.2 Trust). The two fields are, despite their proximity, treated separately in most studies (Figure 1.2). They are distinct from each other and draw from separate reservoirs. The nature of shear wave splitting, as a measurement along a path, allows us to treat both fields together.

\section{Rotokawa geothermal field}

Rotokawa geothermal field itself is thought to be approximately 20,000 years old (Krupp and Seward 1987; Vucetich and Howorth 1976) and is located in the central TVZ (McNamara et al. (2016)). The field itself (Figure 1.2) covers $28 \mathrm{~km}^{2}$ defined 


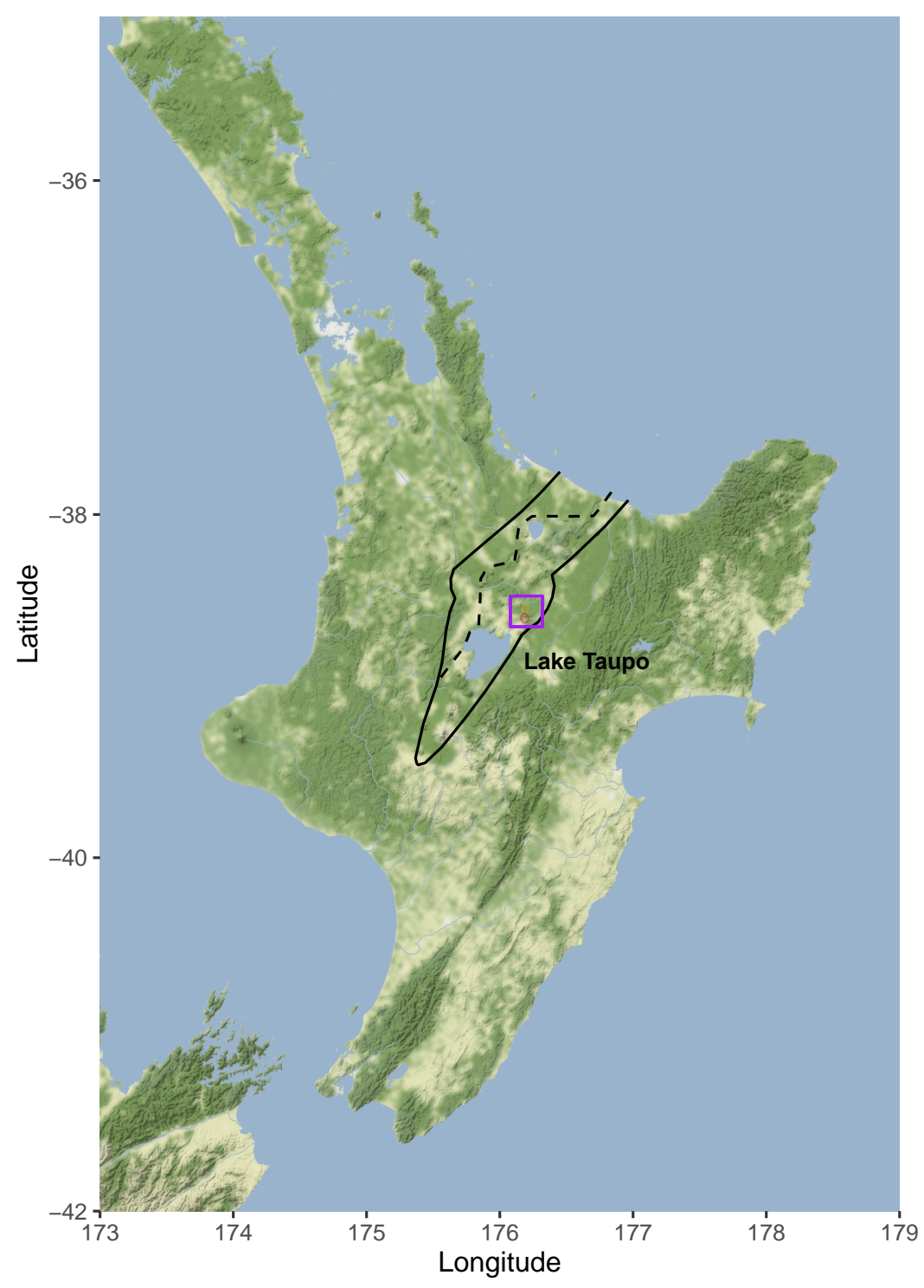

Figure 1.1: Geothermal field locations with respect to the North Island of New Zealand. The purple rectangle indicates the extent of Figure 1.2. The onshore Taupo Volcanic Zone is outlined in black with the dashed line representing the northern extent of the Young TVZ (Wilson et al. 1995). 


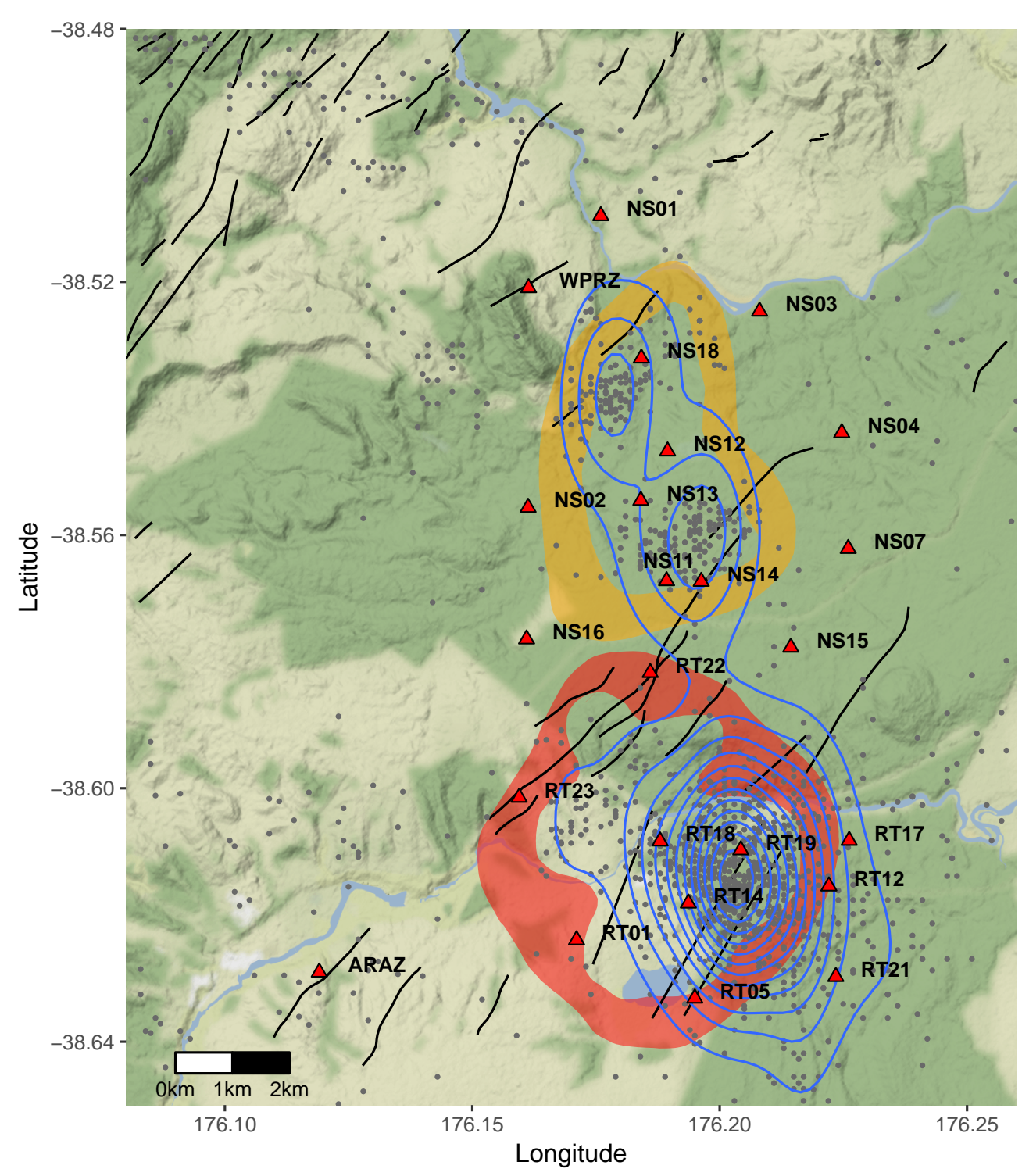

Figure 1.2: Rotokawa (south, red) and Ngatamariki (north, orange) fields outlined by their resistivity boundaries determined by Risk (2000) and Boseley et al. (2010) respectively. Red triangles represent seismometer sites and grey points show earthquakes which gave at least one high grade shear wave splitting measurement. The contours represent the $2 D$ kernel density estimate of earthquakes locations. Solid lines represent active faults traces (see GNS Science Active Faults Database and Wallis et al. (2013)). 
by resistivity boundary (Risk 2000). The field has 175 MWe (megawatt electrical) of installed capacity and makes up a significant portion of geothermal electricity generation (as well as total electricity generation) in New Zealand (Hernandez et al. 2015; Carey et al. 2015). Of the 174 MWe of installed capacity, 140 MWe is from the Nga Awa Purua Power Plant which was commissioned in 2010 and 34 MWe from the Rotokawa binary plant commissioned in 1997 and expanded in 2002 (Carey et al. 2015).

In Rotokawa, andesite lava and breccias, $\sim 2 \mathrm{~km}$ thick, overlie a greywacke basement (Bowyer and Holt 2010).

\section{Ngatamariki geothermal field}

Ngatamariki geothermal field is located north of Rotokawa and covers approximately 7-12 $\mathrm{km}^{2}$ as defined by its resistivity boundary (Boseley et al. 2010) and is shown in Figure 1.2. In 2013 an 82 MWe binary plant was opened and remains the only power plant (Carey et al. 2015).

Approximately $3 \mathrm{~km}$ of mostly rhyolitic ignimbrites overlies a greywacke basement (Chambefort et al. 2014).

\subsection{Previous studies}

\subsubsection{Shear wave anisotropy studies}

Shear wave anisotropy studies have been carried out numerous times within the volcanic areas of the TVZ. These studies use the shear wave splitting technique (described in Chapter 2) to measure the shear wave anisotropy as we do in this study. However, apart from several preliminary studies, geothermal areas within the TVZ have not been investigated using this method. 
We have also published, in proceedings, preliminary results and interpretation for the dataset we investigate in this thesis (Mroczek et al. 2016). We include this paper in Appendix F.

\section{Shear wave splitting in the TVZ}

There are two unpublished feasibility studies using shear wave splitting at Rotokawa by Unglert (2009) and Greenbank (2014). While robust conclusions about the anisotropy cannot be drawn from these studies they do show that shear wave splitting can be measured and interpreted in geothermal reservoirs within the TVZ.

In the Tongariro Volcanic area (southern TVZ) shear wave splitting studies have been carried out numerous times (e.g. Gerst and Savage 2004; Savage et al. 2010a; Johnson 2011; Johnson and Savage 2012; Castellazzi et al. 2015). The methods we apply and adapt in this thesis were first published for studies made in this region; Savage et al. (2010a) for automatic shear wave splitting measurements (Section 3.5), Johnson (2011) for delay time tomography (Section 3.9), and Castellazzi et al. (2015) for automatic S-wave picking of waveforms for later automatic shear wave splitting measurements (Section 3.4).

\section{Geothermal}

While published studies have not being carried out in geothermal areas within New Zealand, shear wave splitting studies have been carried out in geothermal fields elsewhere. For example: Evans et al. (1995); Lou et al. (1997); Elkibbi et al. (2005) at the Geysers geothermal field (California), Lou and Rial (1997) at the Coso geothermal reservoir (California), and Rial et al. 2005 at the Krafla geothermal field (Iceland). Shear wave splitting in geothermal is generally interpreted as arising from stress aligned microcracks (Section 2.3) however faults also seem to have some affect (Section 2.3.3). 


\subsubsection{Stress studies}

The prevalent cause of shear wave splitting in the upper crust is stress aligned microcracks. If the cracks present in the reservoir are stress aligned then the fast polarisations measured from shear wave splitting (Chapter 2) should show some agreement with in-situ and regional measurements of maximum horizontal stress.

Three wells in Rotokawa have had in-situ stress measurements made by McNamara et al. (2015). Directions of maximum and minimum horizontal stress were estimated from drilling induced tensile fractures, petal centreline fractures, and borehole breakouts. Figure 1.3 shows these stress estimates for each well (including minimum horizontal stress estimates $\left.+090^{\circ}\right)$. Well locations are shown in Figure 1.3d. Stress can change significantly with depth within the wells (McNamara et al. 2015). RK18L2 and RK32, despite being spatially close to each other, have means twenty degrees apart (Figure 1.3). These in-situ measurements indicate that the direction of maximum horizontal stress $\left(S_{H_{\max }}\right)$ has strong variations within the Rotokawa reservoir. The difference in the orientation of $S_{H_{\max }}$ between RK18L2 and RK32 is interpreted, by McNamara et al. (2015), as being due to activity on the production field fault (PFF, Figure 1.3d).

Townend et al. (2012), from focal mechanisms, find average orientations of maximum horizontal stress to be NE-SW or ENE-WSW north of Lake Taupo (within the TVZ). We treat this as a regional measure of stress and thus expect most orientations, determined from shear wave splitting, to be in this range even if they do not explicitly agree. 


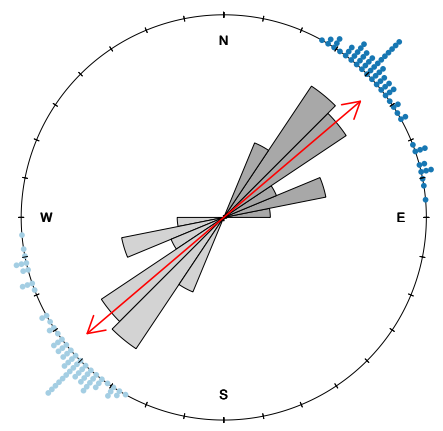

(a) RK18L2. $050^{\circ}$ mean.

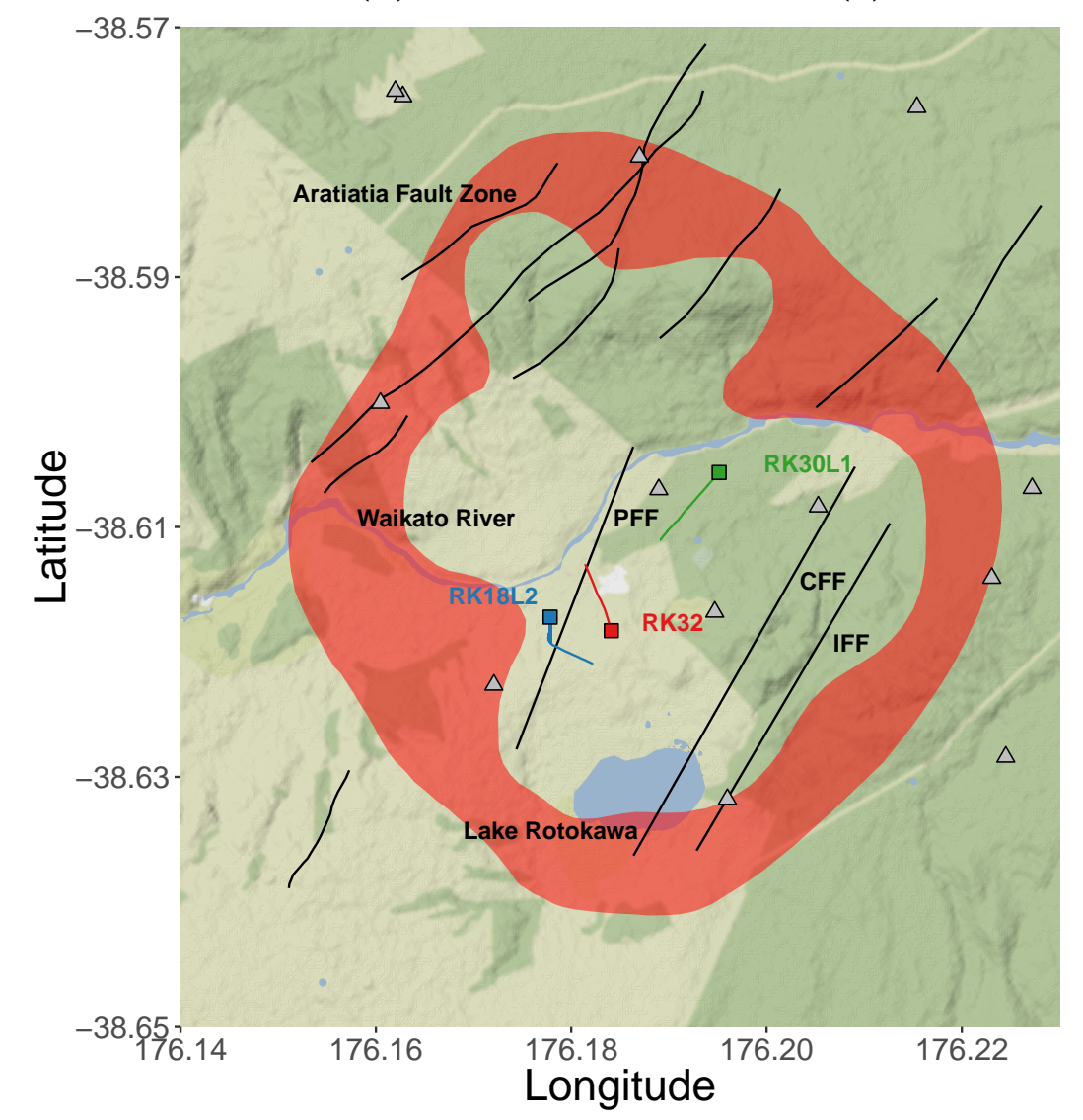

(d) Well head location and well tracks

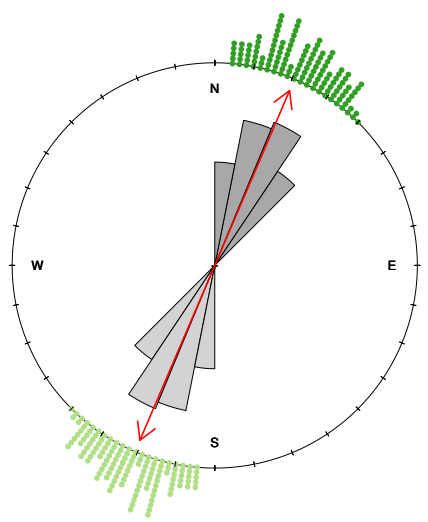

(c) RK30L1. 023 mean.

Figure 1.3: In-situ stress measurements converted to direction of maximum horizontal stress $\left(S_{H_{\max }}\right)$ with their corresponding well tracks for the wells studied by McNamara et al. (2015). The production field fault (PFF), central field fault (CFF), and injection field fault (IFF) are plotted (Wallis et al. 2013). 


\subsection{Earthquake catalogue}

Measurements of seismic anisotropy using the shear wave splitting technique use shear waves originating from earthquakes. Thus the location and amount of seismicity is important for shear wave splitting studies. As both fields are located so close together we use a combined catalogue approach. The GNS earthquake catalogue has been provided by Steven Sherburn.

\subsubsection{Seismometer network}

Earthquakes are recorded on a 22 station network spanning Rotokawa and Ngatamariki (Figure 1.2). Our network is made up of two smaller networks with two additional stations. NS indicates stations part of Mercury's Ngatamariki network and RT indicates stations part of Mercury's Rotokawa network. WPRZ is part of the larger GeoNet network and ARAZ is a Contact Energy station. Most stations are located on flat farmland.

Figure 1.2 shows some stations with multiple names. This is where a station has been moved slightly and assigned a new name. When looking at results per station we merge measurements (for stations less than $100 \mathrm{~m}$ apart) together, while for tomography and spatial averaging the stations are kept separate. NS09 is merged into NS16 and, for this study, is referred to as NS16. NS08 is merged into NS18 and is referred to as NS18.

The network also contains three borehole stations: NS12, NS13 and NS14. Shear wave splitting requires that the orientation of the station be known. Thus, we orient these borehole stations using the method described in Section 3.3.

All RT and NS surface stations are $4.5 \mathrm{~Hz}$ (sampling frequency) Geospace GS11D seismometers. NS12-14 use IESE S10g-4.5 (4.5 Hz) sensors located at $-164 \mathrm{~m}$, $130 \mathrm{~m}$, and $163 \mathrm{~m}$ above sea level respectively (Sherburn and Bourguignon 2014). 
WPRZ uses a $1.0 \mathrm{~Hz}$ Lennartz LE-3Dlite MKII instrument. ARAZ also uses a $1 \mathrm{~Hz}$ instrument (Sercel L4C-3D).

\subsubsection{Location}

Earthquakes in the GNS catalogue were automatically detected and located using the SeisComp3 software package. These locations are based on the P-wave phase pick. S-wave picks are not made. These earthquakes are located with a generic TVZ velocity model.

Earthquakes detected with the Matched Filter method (Section 1.5.3) are located with the program NonLinLoc (Lomax et al. 2014) using a 1D average velocity model from the GNS 3D model for Rotokawa (Rawlinson 2011 p. 77). The P-wave picks of each detection can move slightly (from the template's pick) to maximise the correlation with the template.

The seismicity in Rotokawa is largely confined to one cluster of earthquakes (Figure 1.2) occurring between the production and injection zones (Sherburn et al. 2015). Within Ngatamariki, there are two main clusters of seismicity associated with injection wells (Sherburn and Bourguignon 2014).

Figure 1.4 summarises the event depths of the dataset, with most earthquakes occurring in the 1-3 km depth range. We do not do any magnitude analysis, however almost all these earthquakes fall into the micro-earthquake classification (magnitude $<2.0$ ) with only several above magnitude 3.0 during 2015.

Our earthquake catalogue spans from 1st January to 20th November, 2015. It contains a total of 4,670 unique events. Of these 4,670 events, 2,204 are part of the GNS catalogue. The other 2,466 events are matched filter detections which are described in the following section (Section 1.5.3). Number of events per station are summarised in Table A.3 and A.4. In total, the catalogue contains 52,421 stationearthquake pairs which (if an S-wave pick is successfully made) can be analysed 


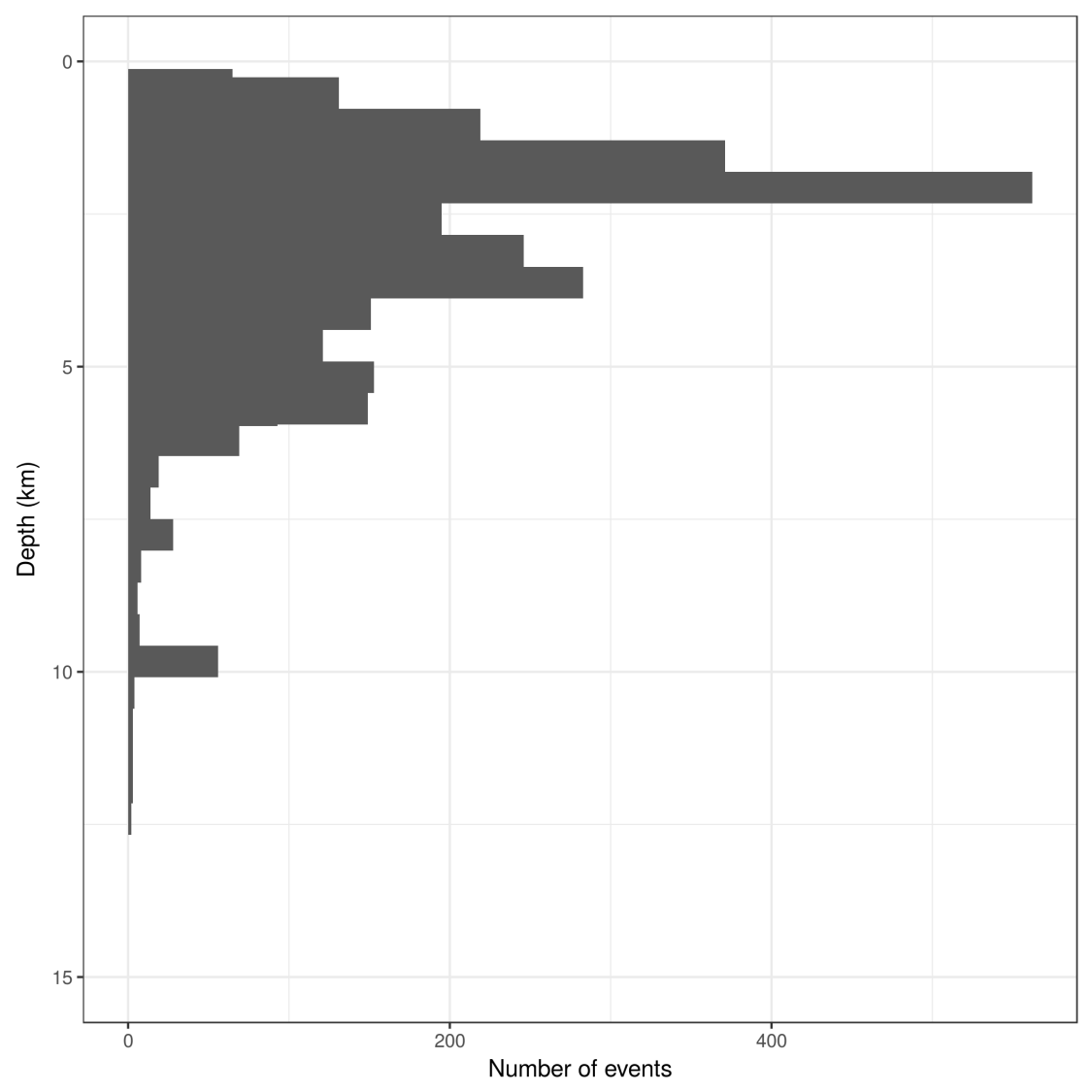

Figure 1.4: Histogram of earthquake depths for events with an S-wave pick on at least one station.

for shear wave splitting. The ratio of earthquakes located in Rotokawa to those in Ngatamariki is approximately 2:1 (for those with an S-wave pick made).

Sherburn et al. (2015) considers that most of the seismicity in Rotokawa is induced by the deep re-injection of condensate and brine as evidence by changes in activity corresponding to changes in injection. Given the proximity between the two fields, it is likely that seismicity in Ngatamariki is induced by similar mechanisms whereby the pre-fractured rock is contracted by cool re-injected fluids allowing fractures to reactivate (Sherburn et al. 2015). 


\subsubsection{Matched filter detection}

The matched filter detections in our catalogue are made by current $\mathrm{PhD}$ student Chet Hopp and are published in proceedings (Hopp et al. 2016). The Matched Filter method of detecting earthquakes relies on waveform cross correlation of a known (template) event with continuous seismic records (Chamberlain et al. 2014). When a detection statistic (the sum of cross correlation coefficients on each channel) reaches a threshold value (8 times the mean mean absolute deviation of the statistic) a detection is made. Hopp et al. (2016) uses the EQcorrscan Python package (Chamberlain et al. 2014) to apply the matched filter technique.

The matched filter method is particularly good at detecting signals on continuous data with high levels of noise (Gibbons and Ringdal 2006), such as geothermal areas with high levels of natural (e.g. Clacy 1968) and anthropogenic noise related to production. This method proved to be very effective at Rotokawa and Ngatamariki with Hopp et al. (2016) detecting 21,100 additional micro-seismic events using 637 templates from the GNS catalogue.

Most events detected using the matched filter method are of poor quality (hence their failure to be detected by more conventional methods). The requirement for a detection to be made, for the 21,100 events, is that the event must have a correlation coefficient of $\geq 0.3$ on at least six stations. We increase this threshold to $\geq 0.5$ on at least nine stations to reduce the catalogue to a more manageable size (removing lower quality events). This reduces the number of detections from 21,100 to the 2,466 we use in this study. These 2,466 detections have comparable, but slightly lower, successful picking and splitting rates to the GNS catalogue (Table A.4). Self detections are removed based on time proximity to their template events. 


\section{Chapter 2}

\section{Seismic anisotropy \& shear wave splitting}

\subsection{Overview}

Seismic anisotropy is a material property in which a seismic wave's velocity is dependent on its direction of propagation and the anisotropic symmetry of that system. Seismic anisotropy can arise from a number of causes, such as alignment of crystals, lithology (e.g. aligned grains), structures (e.g. fine layering), and stress aligned cracks (Crampin and Lovell 1991). The splitting of shear waves is diagnostic of seismic anisotropy and has been observed throughout the crust (e.g. Peacock et al. (1988), Silver (1996), Cassidy and Bostock (1996)) including geothermal areas (e.g. Evans et al. (1995), Lou et al. (1997)).

Shear wave splitting occurs when a shear wave enters an anisotropic medium and is split because the component polarised in one direction travels faster than the orthogonal component (Savage 1999). Shear wave splitting is characterised by two variables: Polarisation of the leading wave (fast polarisation), $\phi$, controlled by the anisotropic symmetry system and its orientation, and delay time, $\delta t$, between the 
arrival of the fast and orthogonal polarisations (Savage 1999). The fast polarisation is an axial quantity. It is an axis (e.g. N-S) rather than a direction (e.g. N) and requires special treatment in circular statistics (Section 3.2.1).

\subsection{Theoretical background}

Here we summarise the theoretical basis of a shear wave anisotropy as presented in Chapter 2 of Babuska and Cara (1991).

To calculate the seismic velocities in a medium, the elastic properties of that medium must be known or approximated. We use the formulations of Hudson (1980, 1981) to determine the elastic properties of a cracked solid (detailed in Section D.1).

\subsubsection{Hexagonal Symmetry}

Anisotropic materials vary in complexity depending on their degree of symmetry (represented in their elasticity tensor). For example triclinic minerals, such as plagioclase, with a low degree of symmetry have 21 independent elastic coefficients (Babuska and Cara 1991). For materials with a higher degree of symmetry the complexity of the system reduces. In the Earth, the most applicable is hexagonal symmetry which approximates many real situations and has five independent terms (Babuska and Cara 1991). Fluid-filled cracks exhibit this hexagonal symmetry. By varying the direction of propagation ( $\hat{\mathbf{n}})$ of a wave through an anisotropic material one can calculate the expected velocities and polarisations of that wave.

\subsubsection{Velocity in an anisotropic material}

To demonstrate how shear wave splitting occurs, we determine seismic velocities for a plane wave travelling through a hexagonal anisotropic medium following Babuska and Cara (1991). This example is equivalent (but not identical) to a wave travelling 
vertically through vertically aligned cracks. The axis of symmetry can be adjusted by rotating the elasticity matrix (e.g. Section D.2).

For the hexagonal case there are five independent elastic constants $(A, N, F, C, L)$ (Love 1927) and the elasticity matrix is given by:

$$
C_{\text {elasticity }}=\left[\begin{array}{cccccc}
A & A-2 N & F & 0 & 0 & 0 \\
A-2 N & A & F & 0 & 0 & 0 \\
F & F & C & 0 & 0 & 0 \\
0 & 0 & 0 & L & 0 & 0 \\
0 & 0 & 0 & 0 & L & 0 \\
0 & 0 & 0 & 0 & 0 & N
\end{array}\right]
$$

The elasticity matrix $\left(C_{\text {elasticity }}\right)$ is a representation of the full $4^{\text {th }}$ order stiffness tensor $c_{i j k l}$ (which depends on the local elastic properties of the material) such that:

$$
C_{\text {elasticity }}=\left[\begin{array}{llllll}
c_{1111} & c_{1122} & c_{1133} & c_{1123} & c_{1113} & c_{1112} \\
c_{2211} & c_{2222} & c_{2233} & c_{2223} & c_{2213} & c_{2212} \\
c_{3311} & c_{3322} & c_{3333} & c_{3323} & c_{3313} & c_{3312} \\
c_{2311} & c_{2322} & c_{2333} & c_{2323} & c_{2313} & c_{2312} \\
c_{1311} & c_{1322} & c_{1333} & c_{1323} & c_{1313} & c_{1312} \\
c_{1211} & c_{1222} & c_{1233} & c_{1223} & c_{1213} & c_{1212}
\end{array}\right]
$$

In the case of isotropy there are only two independent elastic constants. This elasticity matrix simplifies to the isotropic case when $A=C=\lambda+2 \mu, A-2 N=F=\lambda$ and $L=N=\mu$ where $\lambda$ and $\mu$ are the usual Lamé parameters .

The equation of elastodynamics in a homogeneous anisotropic medium is given by

$$
\partial_{j} \sigma_{i j}=\rho \partial_{t}^{2}\left(u_{i}\right)
$$

for $i, j=1,2,3$, where $\partial_{j}$ is the spatial derivative relative to $x_{j}\left(\frac{\partial}{\partial x_{j}}\right), \partial_{t}$ is a time derivative, and $\rho$ is the density. $\sigma_{i j}$ are components of the second-order stress tensor 
which, through Hooke's Law, correspond to the second order strain tensor $\left(\epsilon_{k l}\right)$ by

$$
\sigma_{i j}=c_{i j k l} \epsilon_{k l}
$$

for $i, j, k, l=1,2,3$. By substituting (2.4) into (2.3) we get

$$
c_{i j k l} \partial_{j}\left(\epsilon_{k l}\right)=\rho \partial_{t}^{2}\left(u_{i}\right)
$$

The solution to (2.5) for a plane wave (e.g. Stein and Wysession 2009) is given by:

$$
\mathbf{u}(\mathbf{r}, t)=\mathbf{a} f(t-\hat{\mathbf{n}} . \mathbf{r} / c)
$$

where $\mathbf{u}(\mathbf{r}, t)$ gives the motion of a particle located at position $\mathbf{r}$ and at time $t . \mathbf{u}(\mathbf{r}, t)$ is equal to $\mathbf{a}$, a vector of amplitude and polarity of the wave, multiplied by a function of time, location and phase velocity $(c)$ as well as a unit vector perpendicular to the phase surface $(\hat{\mathbf{n}})$ which corresponds to the direction of propagation of the wave.

In the case of small strain

$$
\epsilon_{k l}=\left(\frac{\partial u_{k}}{\partial x_{l}}+\frac{\partial u_{l}}{\partial x_{k}}\right) / 2
$$

by substituting (2.7) into (2.5) and accounting for the symmetry of the strain and elastic tensors $\left(\frac{\partial u_{k}}{\partial x_{l}}=\frac{\partial u_{l}}{\partial x_{k}}=\partial_{k}\left(u_{l}\right)\right)$ we get

$$
c_{i j k l} \partial_{j} \partial_{k}\left(u_{l}\right)=\rho \partial_{t}^{2}\left(u_{i}\right)
$$

We do not evaluate these derivatives here, but by taking the spatial and time derivatives of (2.6) we get

$$
\partial_{t}\left(u_{l}\right)=c^{2} a_{i}
$$

and

$$
\partial_{j} \partial_{k}\left(u_{l}\right)=a_{l} \hat{n}_{j} \hat{n}_{k}
$$

Substituting (2.9) and (2.10) into (2.8) and rearranging we get

$$
a_{l} \frac{c_{i j k l} \hat{n}_{j} \hat{n}_{k}}{\rho}=c^{2} a_{i}
$$


where the left hand side makes up the elements of the Christoffel matrix which is given by

$$
m_{i l}=\frac{c_{i j k l} \hat{n}_{j} \hat{n}_{k}}{\rho}
$$

or, in long form

$$
m_{i l}=\sum_{k=1}^{3} \sum_{j=1}^{3} \frac{c_{i j k l} \hat{n}_{j} \hat{n}_{k}}{\rho}
$$

It is trivial to write down the Christoffel matrix as elements of $c_{i j k l}$ are known. The eigenvalues of the Christoffel matrix give the phase velocities of the seismic waves and the corresponding eigen-vectors give the polarisation of that wave (a from (2.6)).

To illustrate this for hexagonal anisotropy with the axis of symmetry in the $x_{3}$ direction (in the usual Cartesian coordinate system) for a wave propagating in the $x_{1}$ direction. The unit vector in the $x_{1}$ direction is equal to

$$
\hat{\mathbf{n}}=\left[\begin{array}{l}
1 \\
0 \\
0
\end{array}\right]
$$

Then we can take (2.1) (whose entries (2.2) are components of $c_{i j k l}$ ) and produce the Christoffel matrix

$$
m_{i j}=\frac{1}{\rho}\left[\begin{array}{ccc}
A & 0 & 0 \\
0 & N & 0 \\
0 & 0 & L
\end{array}\right]
$$

The eigenvalues $(\lambda)$ and (unit) eigenvectors (a) of (2.15) are trivial to calculate. Velocity is given by $\alpha$ or $\beta$.

$$
\lambda_{1}=A / \rho \quad \mathbf{a}_{1}=\left[\begin{array}{l}
1 \\
0 \\
0
\end{array}\right] \quad \alpha=\sqrt{\frac{A}{\rho}}
$$




$$
\begin{array}{ll}
\lambda_{2}=N / \rho & \mathbf{a}_{2}=\left[\begin{array}{l}
0 \\
1 \\
0
\end{array}\right] \quad \beta_{1}=\sqrt{\frac{N}{\rho}} \\
\lambda_{3}=L / \rho & \mathbf{a}_{3}=\left[\begin{array}{l}
0 \\
0 \\
1
\end{array}\right] \quad \beta_{2}=\sqrt{\frac{L}{\rho}}
\end{array}
$$

These are three independent sets of eigenvalues/vectors. The first, (2.16), is polarised in the same direction as the wave is propagating and has a velocity of $\alpha$. This is the P-wave. (2.17) and (2.18) are two waves with their polarisations perpendicular to the propagation direction (i.e. S-waves). The wave described by (2.17) is polarised in the $x_{2}$ direction while $(2.18)$ is polarised in the $x_{3}$ direction. In the case that $N>L$ it follows that $\beta_{1}>\beta_{2}$. This is shear wave splitting, as a wave enters this anisotropic medium it is split into two shear waves polarised perpendicular to each other with differing velocity of $\beta_{1}$ and $\beta_{2}$. The gives rise to the shear wave splitting parameters we measure. Thus, if $\beta_{1}>\beta_{2}$, the polarisation, $(\phi)$, of the leading wave (fast polarisation) is given by the eigenvector $\mathbf{a}_{\mathbf{1}}$ and the time delay between the two waves arises from their differing velocities.

This simplifies to the isotropic velocities (where $A=\lambda+2 \mu, N=L=\mu$ ). $\alpha$ becomes the usual P-wave velocity of $\alpha=\sqrt{\frac{\mu+2 \lambda}{\rho}}$ and the $\beta_{1}$ and $\beta_{2}$ the usual S-wave velocity $\beta_{1}=\beta_{2}=\sqrt{\frac{\mu}{\rho}}$.

Being able to calculate the expected polarisation for a particular material allows us to fit actual shear wave splitting measurements to theoretical crack planes (Section $3.8)$.

The phase velocities and polarisations of the constituent waves can be determined at any direction of propagation by varying $\hat{\mathbf{n}}$. Thus, the solutions of the Christoffel matrix for different propagations can be plotted. Figure 2.1 shows a lower hemisphere (angle preserving) pole plot for propagation through a crack plane striking at $045^{\circ}$. 


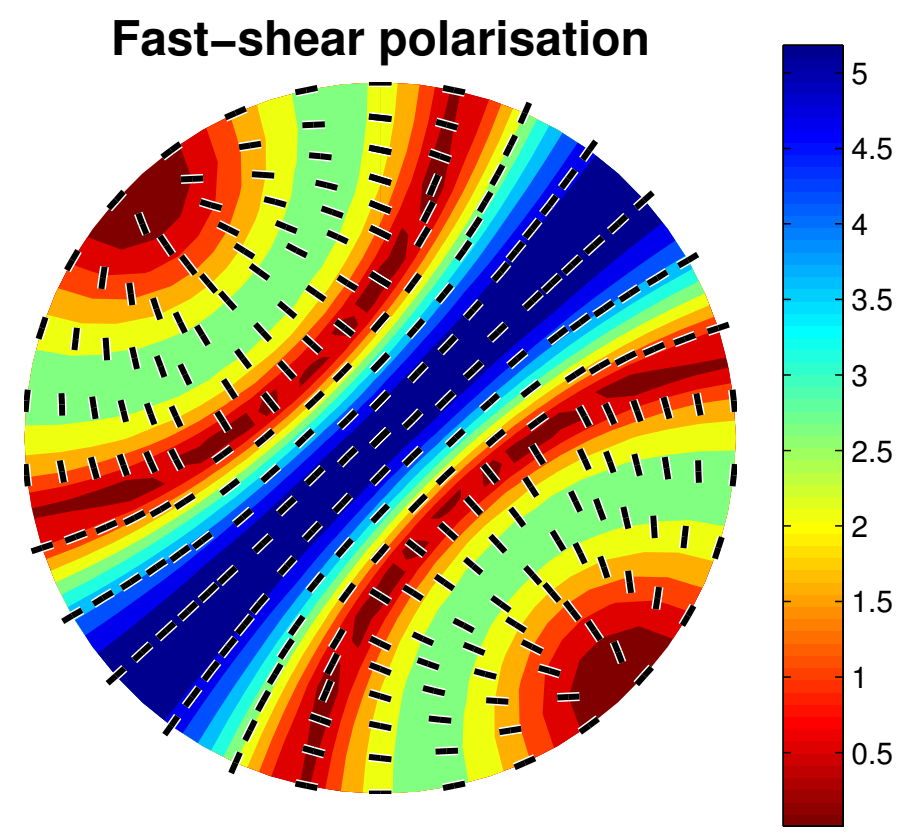

Figure 2.1: Angle preserving pole figure of shear wave splitting parameters for vertical cracks striking $045^{\circ}$. Black lines represent polarisations and contours represent percent anisotropy.

Each point corresponds to a propagation angle with a particular back azimuth and angle of propagation (a single $\hat{\mathbf{n}}$ ) and shows the polarisation (a) of the faster leading wave and the percent anisotropy calculated from the difference in velocity between $\beta_{1}$ and $\beta_{2}\left(k=\frac{\beta_{\max }-\beta_{\min }}{\bar{\beta}} * 100\right)$.

\subsection{Fluid-filled cracks \& shear wave splitting}

\subsubsection{Stress aligned microcracks}

The prevalent cause of shear wave splitting in the upper crust is likely to be fluidfilled microcracks that align with the local orientation of maximum horizontal stress 
$S_{H_{\max }}$ (Crampin and Peacock 2008). Cracks not aligned with $S_{H_{\max }}$ are closed and their fluid forced into vertical cracks aligned with $S_{H_{\max }}$. Thus the fast polarisation aligns with the local direction of $S_{H_{\max }}$ and the delay time gives an indication of the crack density along the path. A shear wave's particle motion is perpendicular to the direction it is travelling, so a vertically travelling shear wave will be sampling the horizontal anisotropy. This form of shear wave splitting is known as azimuthally-aligned shear-wave splitting (Crampin and Peacock 2008). This interaction is illustrated in Figure 2.2. These cracks exhibit hexagonal anisotropic symmetry (Section 2.2.1).

\subsubsection{A note on terminology}

Throughout this thesis we tend to refer to fluid-filled cracks as simply [micro]cracks. By this we mean stress-aligned fluid-filled cracks. This is also known as extensivedilatency anisotropy or EDA-cracks (e.g. Crampin et al. 1984). It is generally assumed that the cracks are saturated (stress-aligned saturated cracks) however dry/unsaturated gas filled cracks can also be referred to as fluid-filled cracks and, unless explicitly stated, we do not make this distinction (i.e. a fluid-filled crack could either be filled with water or gas). In the geothermal context cracks are mostly referred to as fractures.

Fast polarisation, $\phi$, (polarisation of the leading wave) is also known as fast direction or fast azimuth. We refer to $\phi$ as fast polarisation throughout this thesis to emphasise that it is a descriptor of the shear wave.

\subsubsection{Faults versus cracks}

Various studies have observed that, when close to faults, measured fast polarisations align with the fault strike (e.g. Evans et al. (1995), Zhang and Schwartz (1994), Zinke and Zoback (2000)). This alignment may be due to fractures or rock fabric resulting from fault parallel shear (Evans et al. 1995). The TVZ is predominantly 


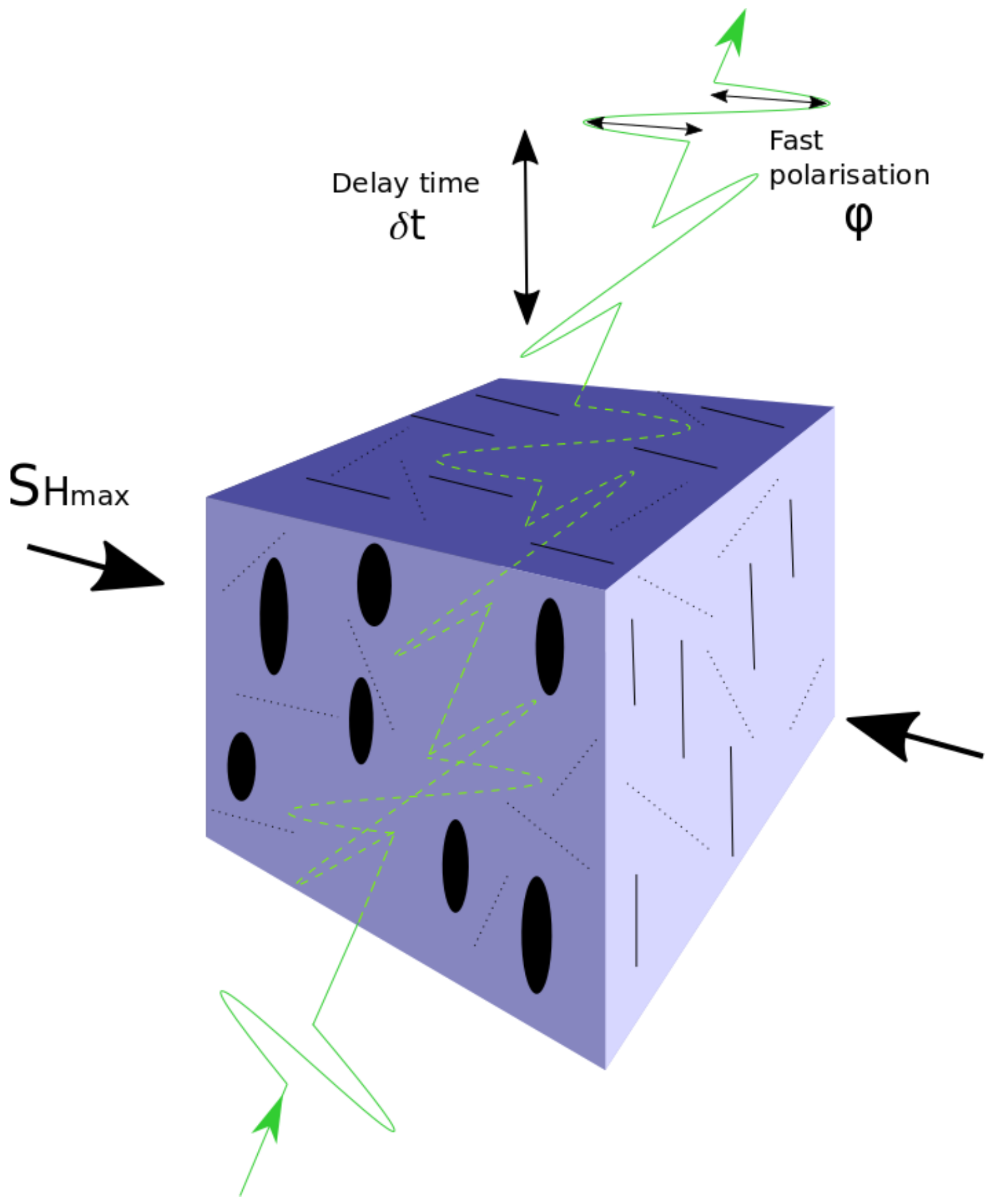

Figure 2.2: Shear wave splitting arising from open microcracks aligned with maximum horizontal stress $\left(S_{H_{\max }}\right)$. An incoming shear wave is split into its fast and orthogonal components. The leading wave is polarised in the same direction ( $\phi)$ as $S_{H_{\text {max }}}$ and crack strike. Delay time $(\delta t)$ separates the fast and slow waves. 
a normal faulting regime (Townend et al. 2012) so $S_{H_{\max }}$ is expected to be parallel to the strike of the faults. Shear wave splitting resulting from either cracks or faults may be indistinguishable from each other in special cases when the faults align with $S_{H_{\max }}$ (e.g. in a normal faulting regime).

\subsubsection{Crack scale}

There is some ambiguity surrounding the scale of cracks that shear wave splitting measures. For example, many small cracks or fewer larger fractures would give the same crack density (or delay time) as measured by shear wave splitting (Maultzsch et al. 2003). There also is a relationship between frequency of the earthquake record and delay time (e.g. Marson-Pidgeon and Savage 1997) with delay time decreasing with increasing frequency of the seismic waves. Maultzsch et al. (2003) use a poroelastic model based on squirt-flow mechanism in fractured porous rock, of Chapman (2003), to suggest that this frequency dependence is sensitive to the length scale of the fractures. Their model also gives invariant polarisations with frequency, however the input only contained fractures of one strike. We know that, at least in Rotokawa, there are subsets of cracks striking in different directions (McNamara et al. 2015). The scale of fractures measured by shear wave splitting in this study most likely falls into the microcrack classification with lengths on the order of centimetres and likely much smaller. Cracks/fractures of varying scale have been observed in Rotokawa (Siratovich et al. 2014; Massiot et al. 2015).

\subsubsection{Evidence for fluid-filled cracks}

In the upper crust the majority of seismic anisotropy measured by the shear wave splitting method (above small earthquakes) is from stress aligned microcracks (Crampin and Peacock 2008). 
It is usual to assume that anisotropy measured in the upper crust originates from aligned fluid-filled cracks (e.g. Crampin and Booth (1985), Volti and Crampin (2003), Evans et al. (1995), Unglert et al. (2011), Castellazzi et al. (2015) and many others). As such, we assume that the majority of the anisotropy we measure at both Rotokawa and Ngatamariki is the result of aligned cracks or fractures present within the reservoirs. If this is true we should observe a correlation between delay time per kilometre and $\mathrm{P}$-wave to $\mathrm{S}$-wave ratios.

\subsection{Further anisotropic considerations}

\subsubsection{Propagation angle}

Fast polarisations are controlled by both the anisotropic medium and the propagation direction, but for many systems the fast directions are similar for a wide range of propagation angles (e.g. Babuska and Cara (1991)). This is an approximation valid for shear waves travelling sub-perpendicular to the crack plane. Outside of this range, fast polarisation orientations can instantaneously switch $90^{\circ}$ and delay times can vary rapidly due to the hexagonal symmetry of the fluid-filled cracks (Section 2.2.1, Figure 2.1). Thus, for the likely case where cracks are non-vertical and propagation angles are not sub-vertical, simply treating fast polarisation as being parallel to crack orientation (and $S_{H_{m a x}}$ ) is not sufficient. To address this, we find the best fitting crack plane for clusters of fast polarisations (Section 3.8).

\subsection{2 $90^{\circ}$ flips}

Due to changing the shape of fluid-filled cracks it is possible that sufficiently high pore pressures could cause measured polarisations to undergo a " $90^{\circ}$-flip", from parallel, to orthogonal to maximum horizontal stress (Crampin and Zatsepin 1997; Crampin 
and Peacock 2005).

Measurements $90^{\circ}$ different from expected could be due to these " $90^{\circ}$-flips", cycle skipping (described in Section 3.6), or shallow propagation angles. We choose to interpret measurements that appear to be flipped $90^{\circ}$ as resulting from shallow propagation angles which carry information we can use in fitting theoretical crack planes (Section 3.8). Visually, this can be seen in Figure 2.1 where polarisations switch very rapidly from $045^{\circ}$, with the crack strike, to normal to the crack strike. Steeper propagation angles can also result in this switch if the symmetry axis of the cracks is not horizontal.

Measurements flipped by either cycle skipping or pore-pressure amount to statistical noise in our crack fitting process. 


\section{Chapter 3}

\section{Methodology}

\subsection{Methods Summary}

Here we summarise the work flow of the methodology section from the earthquake catalogue through to best fitting crack planes and delay tomography.

1) We begin with a catalogue of located earthquakes, from January 2015 through to November 2015, across the region encompassing Rotokawa and Ngatamariki geothermal fields (Section 1.5).

2) Shear wave splitting measurements require that stations be correctly orientated with North. For an incorrectly oriented station (in our case the borehole stations) the fast polarisations and initial S-wave polarisation will have a fixed offset equal to the mis-orientation. To account for this we use the Rayleigh wave method for orienting stations (described in Section 3.3).

3) Shear wave splitting measurements require the S-wave phase to be picked. Pwave picks are either made by GNS or with the matched filter detection. S-wave picks have not been made so, to pick the required S-wave phase, we use the spickerC automatic picker (Section 3.4). 
4) Once S-wave picks have been made, the automatic shear wave splitting measurements are run using MFAST (Section 3.5).

5) To help rule out the migration of seismicity as a cause for changing shear wave splitting parameters, we use a path clustering method described in Section 3.7. This produces clusters of earthquakes for single stations where earthquakes within a cluster have similar station-event paths.

6) Once shear wave splitting measurements have been made and the earthquakes have been clustered into groups based on their station-earthquake paths (5), we then fit the shear wave splitting fast polarisations and delay times to those of a theoretical crack plane (Section 3.8).

7) Depending on the angle of propagation and the orientation of the cracks being sampled, fast polarisation and time delay can vary dramatically. For delay time tomography and spatial averaging of fast polarisations to be effective this variation has to be accounted for. We achieve this by fitting the groups of path clustered shear wave splitting measurements to theoretical crack planes (6) which allows us remove measurements which would not be consistent in the tomography. In Section 3.9 we describe the process of the tomography and spatial averaging.

8) While not strictly related to shear wave splitting, P-wave to S-wave velocity ratios $\left(v_{P} / v_{S}\right)$ can indicate what fluid is present in the cracks (whether it be saturated or dry) and potentially show some relationship with delay time. Since both $\mathrm{P}$-wave and S-wave picks are required for shear wave splitting, calculating $v_{P} / v_{S}$ is trivial (Section 3.10). 


\section{$3.2 \quad$ Statistical tests and methods}

\subsubsection{Circular statistics}

Circular statistics is the branch of statistics which deals with circular quantities (i.e. directions). Although most concepts from traditional statistics transfer to circular statistics the treatment of circular data is different. For analysis, circular data can be treated as angles (as we do here) or complex numbers (Mardia and Jupp 2009). Methods for calculating various descriptive statistics (e.g. the mean of a sample) differ for circular data. We take care to use the correct circular methods (e.g. those in Mardia and Jupp (2009), and Pewsey et al. (2013)) for treatment of our fast polarisations.

The majority of circular quantities in this study (notably polarisation) are axial. An axial variable is an axis (e.g. North-South) rather than a simple direction (e.g. North). A unit vector $\mathbf{x}$ to a point on a circle can be described by its Cartesian coordinates as $\mathbf{x}=\left(\begin{array}{c}\cos \theta \\ \sin \theta\end{array}\right)$ where $\theta$ is the angle from $0^{\circ}$ in a polar coordinate system. For shear wave splitting measurements we already have values of $\theta$ (actually $\theta / 2$, see axial data below).

Here we provide an example of how the mean direction for a circular variable is calculated (following Mardia and Jupp (2009)) by treating directions as unit vectors from the centre of the circle to a point mass on the circle's circumference. The mean of a set of directions is equivalent to the centre of mass of these unit masses. The usual equation for the centre of mass of point masses is given by:

$$
\mathbf{R}=\frac{1}{M} \sum_{i=1}^{n} m_{i} \mathbf{x}_{i}
$$

where $M=\sum_{i=1}^{n} m_{i}$ and $m_{i}$ and $\mathbf{x}_{i}$ are the mass and coordinate of the $i_{t h}$ point respectively. We apply this equation to our unit vectors firstly by assuming $m_{i}=1$ 
(all our points have equal weight) and by extension $M=n$ (where $n$ is the number of point masses/directions) the equation becomes:

$$
\mathbf{R}=\frac{1}{n} \sum_{i=1}^{n} \mathbf{x}_{i}
$$

The coordinates of $\mathbf{x}=\left(\begin{array}{c}\cos \theta \\ \sin \theta\end{array}\right)$ thus

$$
\mathbf{R}=\frac{1}{n} \sum_{i=1}^{n}\left(\begin{array}{c}
\cos \theta_{i} \\
\sin \theta_{i}
\end{array}\right)
$$

In Cartesian coordinates $\mathbf{R}$ is given by $(\bar{C}, \bar{S})$, thus

$$
\begin{gathered}
\bar{C}=\frac{1}{n} \sum_{i=1}^{n} \cos \theta_{i} \\
\bar{S}=\frac{1}{n} \sum_{i=1}^{n} \sin \theta_{i}
\end{gathered}
$$

We can convert $\bar{C}$ and $\bar{S}$ back to an angle by

$$
\bar{\theta}=\operatorname{atan} 2(\bar{S}, \bar{C})
$$

where $\bar{\theta}$ is the mean direction of the circular data.

We can also calculate $\bar{R}$, the Pythagorean length of $\mathbf{R}$, with $\bar{R}=\sqrt{\bar{C}^{2}+\bar{S}^{2}}$ which is known as the mean resultant length and gives a measure of concentration of points around the mean. The mean resultant length is used by the Rayleigh test (Section 3.2.3) to test for significance of a mean.

\section{Axial data}

Shear wave splitting fast polarisations are an axial quantity and thus have the property that $\phi=x \bmod 180^{\circ} \in\left(-90^{\circ}, 90^{\circ}\right)$. This is effectively a $180^{\circ}$ ambiguity 
(e.g. $1^{\circ}$ is the same as $\left.181^{\circ}\right)$. Where as, for normal circular quantities $\theta=x$ $\bmod 360^{\circ} \in\left(-180^{\circ}, 180^{\circ}\right)$ is true.

$2 \phi$ fulfills the criteria of $\theta$ so, in many statistical operations, an axial quantity can be conveniently treated as a simple direction by doubling the angle value, performing the operation and then halving the result (for example, when calculating a mean).

Fisher (1995) suggests that measures of concentration or spread (e.g. circular concentration) should be left in the double angled form. However, we choose to convert measurements of standard deviation and standard error back to axial quantities as, being descriptive statistics, it is helpful in their visualisation.

\subsubsection{The von Mises distribution}

The von Mises distribution (Figure 3.1) is the circular equivalent of a normal distribution. It is one of the most useful distributions on the circle (Mardia and Jupp 2009) and has been used (in its axial form) for describing fast polarisations from shear wave splitting (e.g. Roman et al. 2011). The non-axial von Mises distribution could also be used to describe the doubled azimuths.

From Mardia and Jupp (2009), the probability density function of the von Mises distribution is defined as:

$$
g(\theta ; \mu ; \kappa)=\frac{1}{2 \pi I_{0}(\kappa)} e^{\kappa \cos (\theta-\mu)}
$$

where $\theta$ is the direction, $\mu$ is the mean, $\kappa$ is the circular concentration, and $I_{0}$ is the modified Bessel function of the first kind and order zero:

$$
I_{0}(\kappa)=\frac{1}{2 \pi} \int_{0}^{2 \pi} e^{\kappa \cos (\theta)} d \theta
$$

\subsubsection{Rayleigh test}

Calculating the mean orientation of a sample of fast polarisations (for example from a individual station) is a routine process when interpreting shear wave splitting results. 


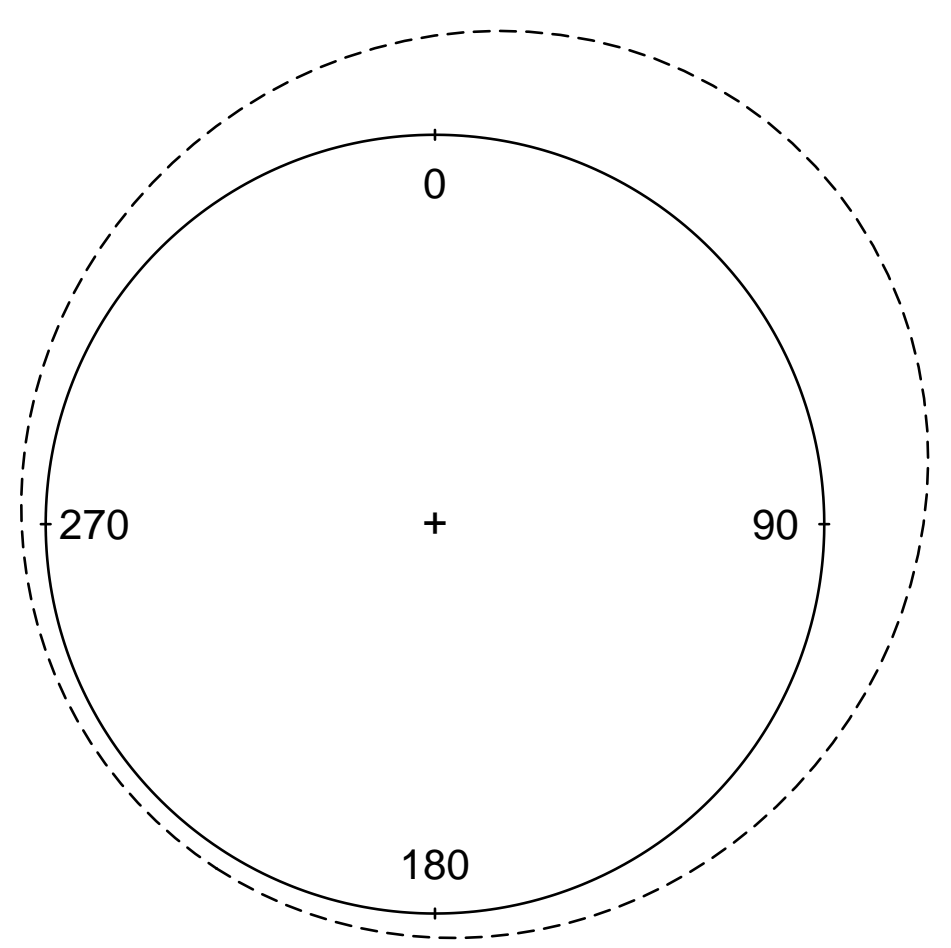

Figure 3.1: The density of a von Mises distribution with $\mu=24^{\circ}$ (mean direction) and $\kappa=1$ (concentration). 
While calculating a mean will always give a result, it is also important to know if the returned mean is statistically significant (i.e. there $i s$ a mean direction). To achieve this we use the Rayleigh test which is a statistical test for circular uniformity. If the concentration of data points around a circle is above a certain threshold then the hypothesis that there is circular uniformity (no dominant mean direction) is rejected (Pewsey et al. 2013). The Rayleigh test is sensitive to unimodal departures from uniformity (i.e. a multimodal distribution could fail the Rayleigh test).

The test relies on the test statistic $n \bar{R}^{2}$ where $\bar{R}$ is the mean resultant length (a measure of concentration around the mean) determined when calculating the mean (Section 3.2.1). When the test statistic is zero the distribution is exactly uniformly distributed. The returned p-value (determination of which is described by Wilkie (1983)) indicates the significance of the test statistic.

\subsubsection{Watson's non-parametric test}

Watson's non-parametric test (Watson et al. 1983) tests the null hypothesis of a common mean direction for two or more samples (Pewsey et al. 2013). So, for example, an extremely low p-value would indicate that the null hypothesis that the two samples have a common mean direction can be rejected.

The test is very useful as it allows us to compare the means of two samples. For example, it can be applied to the fast polarisations from two or more separate stations to test whether the null hypothesis (that their means are the same) can be rejected or not.

Specifically, we use the bootstrap version of the test (to allow smaller sample sizes to be used) following the method of Fisher (1995) (Pewsey et al. 2013). This test is non-parametric; it makes no assumption about the underlying distribution of directions. 


\subsubsection{Pearson's product-moment correlation}

To measure correlations between paired samples (e.g. $v_{P} / v_{S}$ and delay time) we use Pearson's product-moment correlation coefficient. The null hypothesis that the underlying coefficient is zero is tested, which is useful in that it allows us to decide if two variables have some finite correlation.

The correlation, $\rho$, is given by

$$
\rho_{x, y}=\frac{\operatorname{cov}(x, y)}{\sigma_{x} \sigma_{y}}
$$

where $\sigma$ is the standard deviation of two samples or populations $x$ and $y$.

Pearson's product-moment correlation measures linear correlation between two variables and returns a coefficient between -1 and 1 (-1 being perfect negative correlation and 1 being perfect correlation).

We utilise the $\mathrm{R}$ function cor.test() (R Core Team 2013) to measure correlations. We also use the circular version of Pearson's product-moment correlation, from Agostinelli and Lund (2013), for measuring correlation between circular variables.

\subsubsection{Bootstrapping}

In statistics, bootstrapping is where a sample is re-sampled randomly with replacement (the same measurement can be sampled more than once). This re-sampling is repeated a large number of times and the resulting re-sampled datasets can be used for a number of purposes.

We take advantage of this when performing operations on smaller samples sizes (Section 3.2.4), calculation of standard error (Section 3.2.7), and for estimating confidence intervals (Section 3.2.5). 


\subsubsection{Standard error}

Standard error (of the mean) is a statistic that measures how close the sample mean is to the population mean. We calculate the circular standard error using a bootstrap method (3.2.6) by re-sampling (with replacement) the (doubled for axial quantities) angles and calculating the mean of the re-sampled values. This is repeated 9999 times with the mean calculated each time. The standard error is the standard deviation of these means. The standard error is then halved if we are dealing with an axial quantity (such as polarisation). We apply this to axial quantities using the sm.stde function (Section E.2.9).

This method has the advantage of being non-parametric, it makes no assumption about the underlying distribution of directions.

\subsubsection{Hierarchical clustering}

Hierarchical clustering is a method of clustering that compares dissimilarities between objects and puts them into a hierarchy based on how similar or dissimilar they are with each other.

To carry out hierarchical clustering, the algorithm takes a table of distances or dissimilarities (in our case 1-correlation) and returns a dendrogram (e.g. Figure B.7). We use the complete linkage method for our hierarchical clustering, which finds similar clusters by iteratively joining similar clusters and then recalculating their distance at each step (R Core Team 2013). The complete linkage algorithm merges the two clusters with the lowest distance at each step. The distance between the newly merged cluster and an old cluster is equal to the maximum distance between the constituent clusters of the new cluster with the old cluster. We demonstrate this for one iteration in Table 3.1. The algorithm iterates until there is only one cluster.

The result of a hierarchical clustering is plotted in a dendrogram which shows 
Table 3.1: Demonstration of hierarchical clustering with the complete linkage algorithm for one iteration. a $\&$ b have the lowest distance so are merged. The distance this new cluster $(a+b)$ has with $c$ is equal to the maximum distance of $a$ or $b$ with $c$.

\begin{tabular}{l|lll} 
& $\mathrm{a}$ & $\mathrm{b}$ & $\mathrm{c}$ \\
\hline $\mathrm{a}$ & 0 & 0.2 & 0.7 \\
$\mathrm{~b}$ & 0.2 & 0 & 0.5 \\
$\mathrm{c}$ & 0.7 & 0.5 & 0
\end{tabular}

(a) Raw table of distances

\begin{tabular}{l|ll} 
& $\mathrm{a}+\mathrm{b}$ & $\mathrm{c}$ \\
\hline $\mathrm{a}+\mathrm{b}$ & 0 & 0.7 \\
$\mathrm{c}$ & 0.7 & 0
\end{tabular}

(b) After one iteration of clustering

visually the distance at which two objects are merged. The lower down the dendrogram two objects (e.g. our misfit grids) branch off the more similar they are. Applying a cut-off allows us to group together the most similar objects.

To perform hierarchical clustering we use the hclust() function in $\mathrm{R}$ (R Core Team 2013).

\subsection{Station orientation}

Three borehole stations located in Ngatamariki (NS12, NS13 and NS14) are not correctly oriented with north. As a result, all polarisations measured by shear wave splitting have an unknown constant offset equal to the mis-orientation. These stations cannot be accessed physically so other methods to orient them must be used.

Orienting of these stations using a P-wave method (from Stachnik et al. (2012)) was abandoned after accurate P-wave picks for teleseisms could not be made. Instead, here we use the surface wave method of Stachnik et al. (2012) which has been modified for use in orientating ocean bottom seismographs (OBS) in Cascadia (e.g. Lodewyk and Sumy 2015). The modified version runs in an interactive Matlab routine with input data in the SAC format. 


\subsubsection{Rayleigh-Wave method}

The approach of Stachnik et al. (2012) is based on that of Baker and Stevens (2004), which does not rely on accurate knowledge of source parameters. The focus of Baker and Stevens (2004) is to determine the back azimuth of a particular earthquake for a correctly oriented station. For a well located teleseismic event the back azimuth is well known. Thus, if the station is not correctly oriented, the difference between the known back azimuth and the back azimuth determined from the Rayleigh wave polarisation gives you the mis-orientation of the station.

Rayleigh wave particle motion is a retrograde ellipse and thus should theoretically appear only on the vertical and radial components (e.g. Stein and Wysession 2009, Chapter 2).

The first step is rotating the seismogram into radial and transverse coordinates for back azimuths from 0 to $360^{\circ}$. The vertical and horizontal components are $90^{\circ}$ out of phase and the easiest way to correlate the components is to correct for this phase shift. This is achieved using the Hilbert transform on the radial component. The Hilbert transform has a $90^{\circ}$ phase shift, which cancels the phase shift between the components. Thus, the vertical component and the Hilbert transformed radial component have a linear relationship (Stachnik et al. 2012). As the relationship between components is now linear a cross correlation can be applied. The best estimate for back azimuth from the Rayleigh wave polarisation is when the correlation between (Hilbert transformed) radial and vertical components is at a maximum. Almost all Rayleigh waves exhibit retrograde ellipticity, which resolves the $180^{\circ}$ ambiguity in orientation present in body wave methods (Stachnik et al. 2012). Maximum positive correlation corresponds to the "correct" back azimuth as opposed to the maximum negative correlation for the opposite direction. The stronger this correlation, the better the estimate (Baker and Stevens 2004). The mis-orientation (and thus the true orientation) of the station can then be estimated from the difference between 
the back azimuth measured from this method and the actual back azimuth (known from the well located teleseism).

This process is repeated for each of the teleseismic events in the catalogue and each event gives an estimate of the station's true orientation of the north component. These estimates are graded and then averaged to give a final estimate of the station orientation (see Section 3.3.2).

\subsubsection{Processing}

\section{Data Preparation}

Two years of seismic data is used in the orientation (from 2014 to the end of 2015). Earthquakes over magnitude six, occurring between $030^{\circ}$ and $180^{\circ}$ epicentral distance from Rotokawa and Ngatamariki and at less than $100 \mathrm{~km}$ depth, were selected from the USGS catalogue. This gave a total of 185 earthquakes which were cut from the day long waveform records from each borehole station.

Waveforms are bandpass filtered (0.04 to $0.06 \mathrm{~Hz})$ with a $10 \%$ cosine taper. The filter values were chosen by applying a range of filters to a test event and selecting the one which best visually showed the surface waves. Stachnik et al. (2012) apply a band pass filter with a lower frequency band (0.02 to $0.04 \mathrm{~Hz})$. Our seismometers are higher frequency instruments than the OBS studied by Stachnik et al. (2012) and so require different filtering.

\section{Processing Routine}

After the orientation from a particular teleseismic event has been calculated it is given a grade of either "bad", "questionable" or "good". The final orientation estimate is a mean of only individual orientations with a grade of "good". The Matlab routine automatically grades all measurements with correlation of less than 0.5 between the 
radial and vertical components as "bad". When the correlation coefficient is above 0.5 the events are passed to the operator for manual grading. We manually grade measurements with poor signal to noise ratio, where the surface wave energy hard to distinguish from the background, as "bad" (e.g. Figure 3.2). Events with correlations made over a smaller amount of the waveform and those with energy in unexpected regions (perhaps from simultaneous local events) are graded "questionable" (e.g. Figure 3.3). The remaining events with high signal to noise ratio and clear wave forms are graded "good" (e.g. Figure 3.4). For the purposes of the final estimate of orientation "bad" and "questionable" grades are the same (both being ignored). Earthquakes that gave a "good" estimate of orientation on at least one station are given in Table A.1 (for 2014 events) and Table A.2 (for 2015 events). 


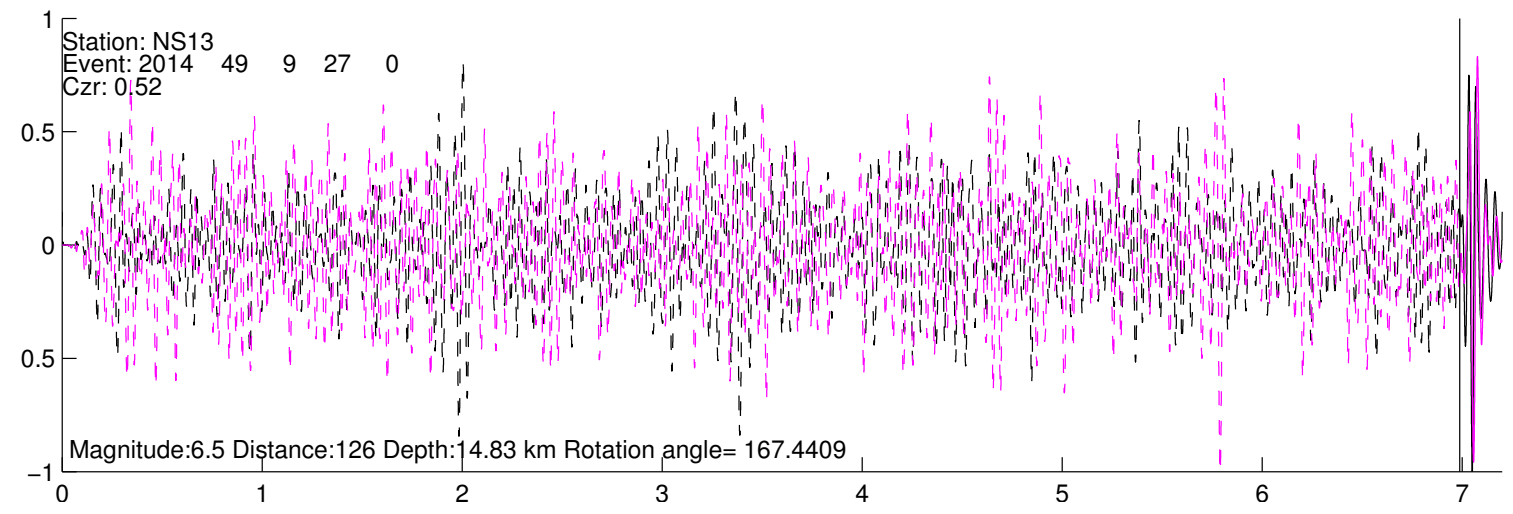

(a) Full window.

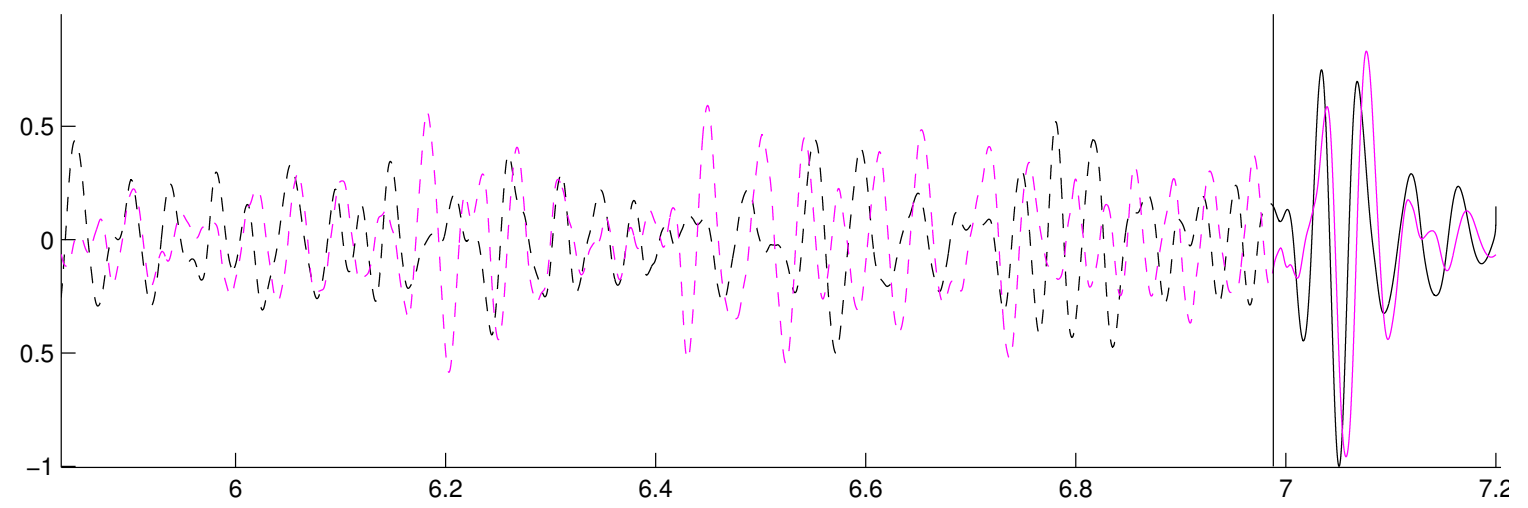

(b) Zoomed window.

Figure 3.2: Example of "bad" orientation measurement. Black and purple components represent vertical and rotated radial (phase shifted) components respectively. The station orientation estimate is calculated for the region after the vertical black line. Correlation of 0.52. 


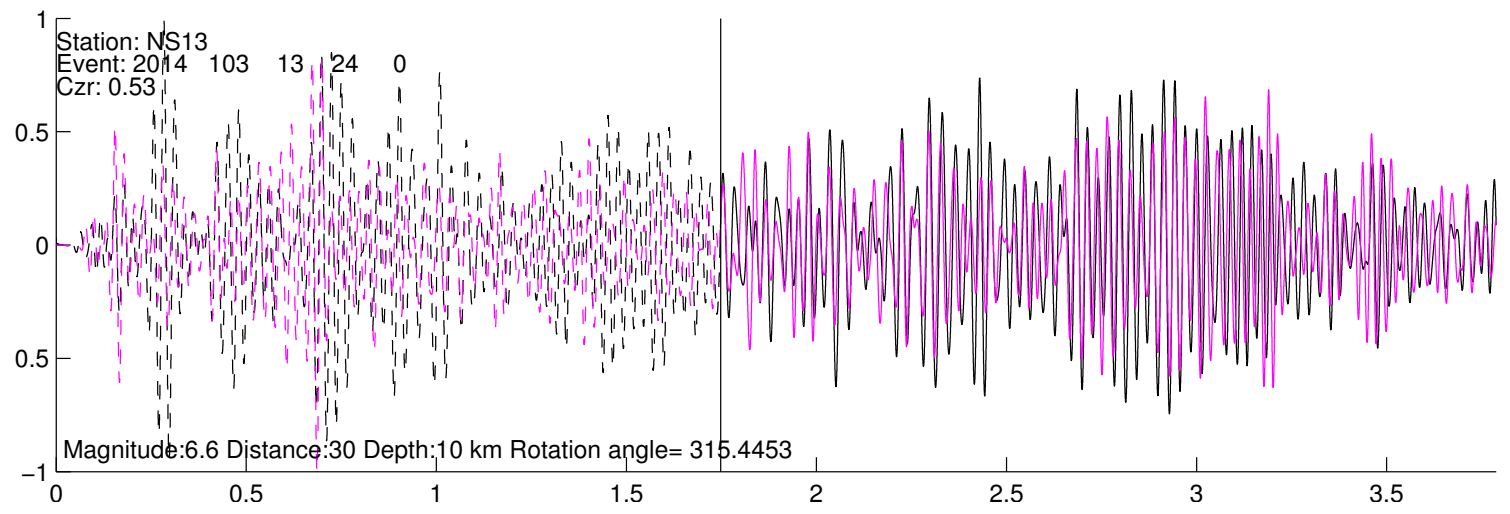

(a) Full window.

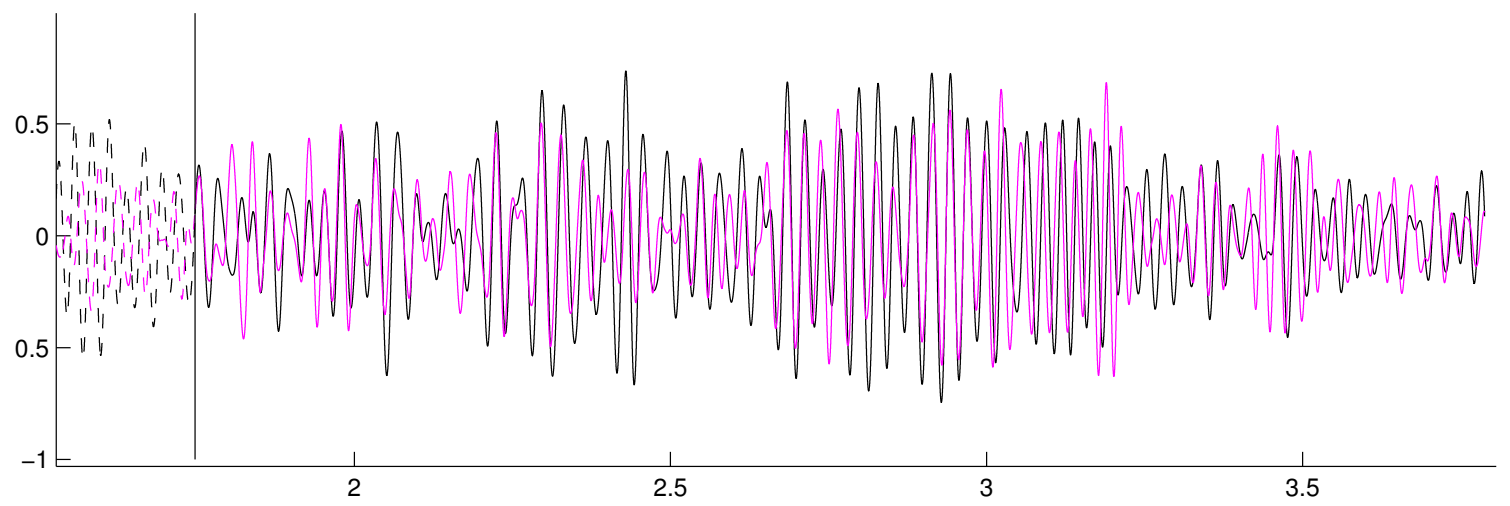

(b) Zoomed window.

Figure 3.3: Example of "questionable" orientation measurement. Black and purple components represent vertical and rotated radial (phase shifted) components respectively. The station orientation estimate is calculated for the region after the vertical black line. Correlation of 0.53. 


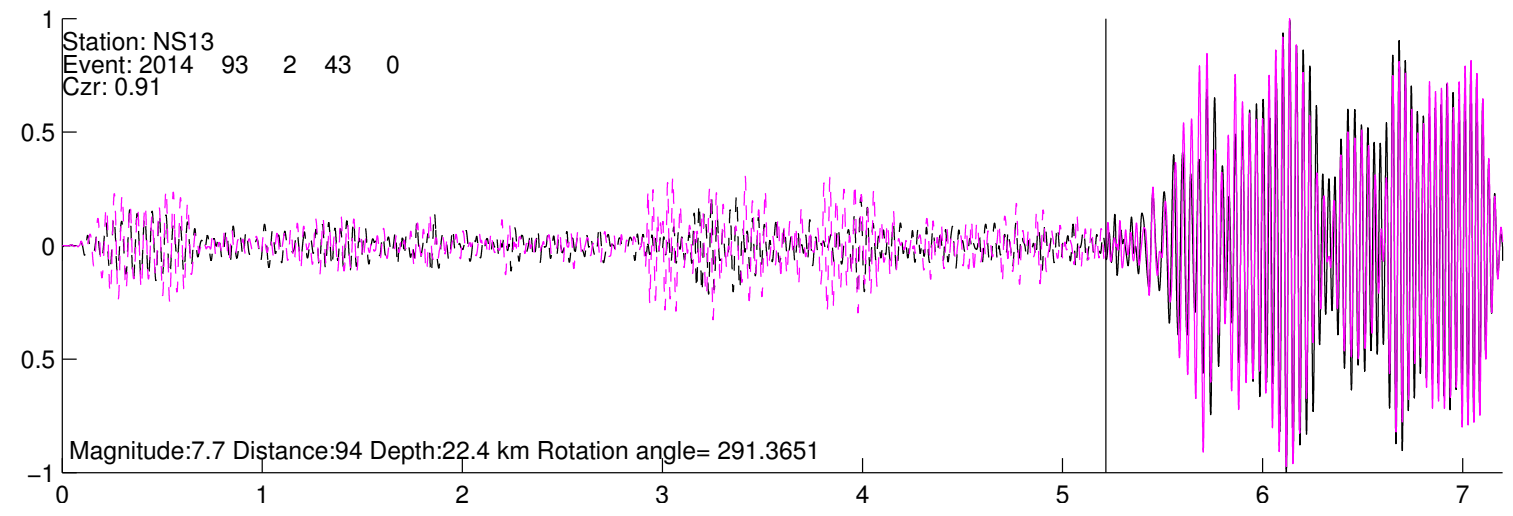

(a) Full window.

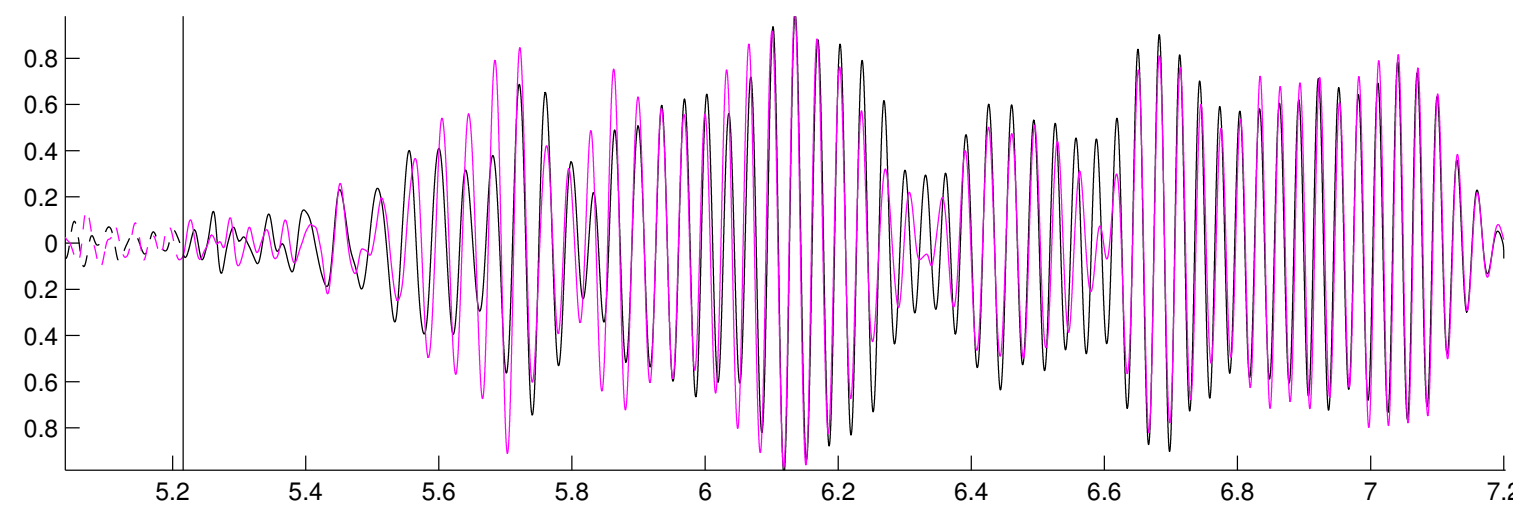

(b) Zoomed window. Note that components are almost identical.

Figure 3.4: Example of "good" orientation measurement. Black and purple components represent vertical and rotated radial (phase shifted) components respectively. The station orientation estimate is calculated for the region after the vertical black line. Correlation of 0.91. 


\subsection{Automatic shear wave picking}

\subsubsection{S-picker overview}

Shear wave splitting measurements require the shear wave phase to be determined. The volume of events in the catalogue make manual picking time prohibitive so instead an automatic phase picker is used, spickerC of Castellazzi et al. (2015).

spickerC uses a combination of three different detection and picking methods: short term average versus long term average ratio (STA/LTA) as described by Allen (1978), polarisation detection based on the approach of Cichowicz (1993) and autoregressive picker using the Akaike Information Criterion (AR-AIC) as described by Leonard and Kennett (1999). The final pick is a weighted average of the three methods with a final grade based on the error estimate and the signal to noise ratio around the pick (Castellazzi et al. 2015). To account for shear wave splitting we use the AR-AIC pick instead of the final pick (Section 3.4.4).

The automatic shear wave picking program (S-picker) was originally written by Diehl et al. (2009). The version used here was modified from version 1.4.0, for use with local earthquakes in New Zealand, by Castellazzi et al. (2015) where S-picker was used in conjunction with MFAST to make automatic picking and splitting measurements at Ruapehu Volcano in New Zealand. Corrections and modifications made to S-picker (updating to version 1.4.0.a) by Castellazzi et al. (2015) are described in Section 3.4.3. This most recent version of S-picker 1.4.0.a, used for processing here, is known as spickerC.

\subsubsection{S-picker methodology}

The following sections provide a summary of the S-picker methodology as given by Diehl et al. (2009).

Before picking, data were filtered with both a Wood-Anderson filter and a high 
pass filter (0.5 Hz second order butterworth). The Wood-Anderson filter integrates to displacement and is used to produce Wood-Anderson seismograms (e.g. Uhrhammer and Collins 1990). Our stations are not broadband and we do not adjust them for their instrument frequency response. The application of the filter in spickerC does not adjust for response magnification. So after Wood-Anderson filtering, while our seismograms are not in Wood-Anderson form, they have still been integrated which acts like an integrating filter. Since lower frequencies are being boosted, the S-wave picking is being made on longer period energy which may be less affected by the scattering of higher frequency incoming waves.

The characteristic function of the seismic signal, used in several of the following methods, is a combination of three (squared) elements; deflection angle, degree of polarisation, and the ratio between transverse and total energy (Cichowicz 1993) for a specified window on a seismogram.

\section{STA/LTA detector}

STA/LTA detector compares the short term average (STA) to the long term average (LTA) in amplitude of the horizontal components (Allen 1978). This is achieved with a comparison between windows for the LTA and STA. This method is sensitive to change in amplitude so it has to be ensured that there is no $\mathrm{P}$ phase within the search window (even if only horizontal components are being used). To avoid this, the coarse window is set using the already picked $\mathrm{P}$ phase and a predicted S-wave arrival. The arrival of the S-phase is expected to occur before the maximum amplitude of the window. A minimum picking approach (Cichowicz 1993) is used where the global minimum of the characteristic function (see the beginning of this section) before the maximum amplitude is selected. 


\section{Polarisation detector}

The polarisation detector also relies on the characteristic function (see the beginning of this section) following the approach of Cichowicz (1993) with an additional weighting parameter. The arrival of the S-phase corresponds with an increase in the S-wave operators of the characteristic function. Much like the STA/LTA detector, the picking algorithm uses characteristic function to select the final pick location. By design, the polarisation filter should be a perfect S-wave picker however it has a lower detection rate than the STA/LTA method due to $\mathrm{S}$ to $\mathrm{P}$ converted waves blurring the S-wave arrival (Castellazzi et al. 2015).

\section{Autoregressive picker}

The AR-AIC method works by approximating non-stationary seismic signals by dividing the time series into segments, each modelled as an autoregressive process where a time series is regressed against itself (Kitagawa and Akaike 1978). These models change after a seismic arrival so arrivals can be picked by finding where the Akaike Information Criterion (Akaike 1973) of the model reaches a minimum. The algorithm used is largely based on that of Takanami and Kitagawa (1988).

This picking method selects different arrivals on the north and south components of the seismogram. As we assume shear wave splitting is occurring, the components will have different arrival times on each of the components. So for a final pick, instead of using and average, we use the earliest AR-AIC pick which is the pick MFAST expects (Castellazzi et al. 2015).

\subsection{3 spicker $C$ modifications}

spickerC contains several changes from S-picker 1.4.0 from Diehl et al. (2009). This section lists the major changes as given by Castellazzi et al. (2015). Apart from 
bug fixes, these changes were based on examining manually analysed events from the Darfield region (Syracuse et al. 2013) and (importantly) the TVZ (Johnson 2011).

- An error was fixed in the STA/LTA function and picking subroutine window.

- Closer station-earthquake spacing and presence of S to P converted waves near S-wave arrivals at some stations lead to modification of the coarse window, searching window, and introducing a clipped amplitude check.

- A check to avoid converted P arrivals was added.

\subsubsection{Grading}

spickerC categorises its automatic picks into classes depending on their assigned error value. The class 0 category is the category with the lowest error. In this study class 0 picks have an error of $\leq 0.3$ seconds and a signal to noise ratio (SNR) of $\geq 3$. Picks in the class0 category are those which then have their shear wave splitting determined. Following the method of Castellazzi et al. (2015), we use the earliest AR-AIC pick from the class0 category. This better approximates manual picks as well as being what is expected for automatic shear wave splitting measurements (Castellazzi et al. 2015).

\subsubsection{Processing parameters}

spicker $C$ was trained using 200 manually picked events from station RT01 in Rotokawa detected from March to June 2013 (Greenbank 2014). RT01 is the station with the most events picked. Figure 3.5 shows the difference between these manual picks and the first AR-AIC pick for picked events for all stations in this time period.

The parameters used in S-picker were chosen in a trial and error process. Initial values were set similarly to those of Castellazzi et al. (2015) (Ruapehu) with some 
modifications based on the change in setting from volcanic to geothermal. The parameters were then varied individually. After a parameter was changed spickerC was rerun. An increase in the number of class0 picks was considered a positive change and the change was kept while no change or a decrease in class0 picks was reverted. Parameters can be found in Table C.1.

Most changes resulted in an increase or decrease of less than ten events. The largest change came from changing the allowable $v_{P} / v_{S}$ band, from 1.5-2.05 (of Castellazzi et al. (2015)) to 1.2-2.2, which almost doubled the number of class0 S-wave picks from 40 to 74 . 


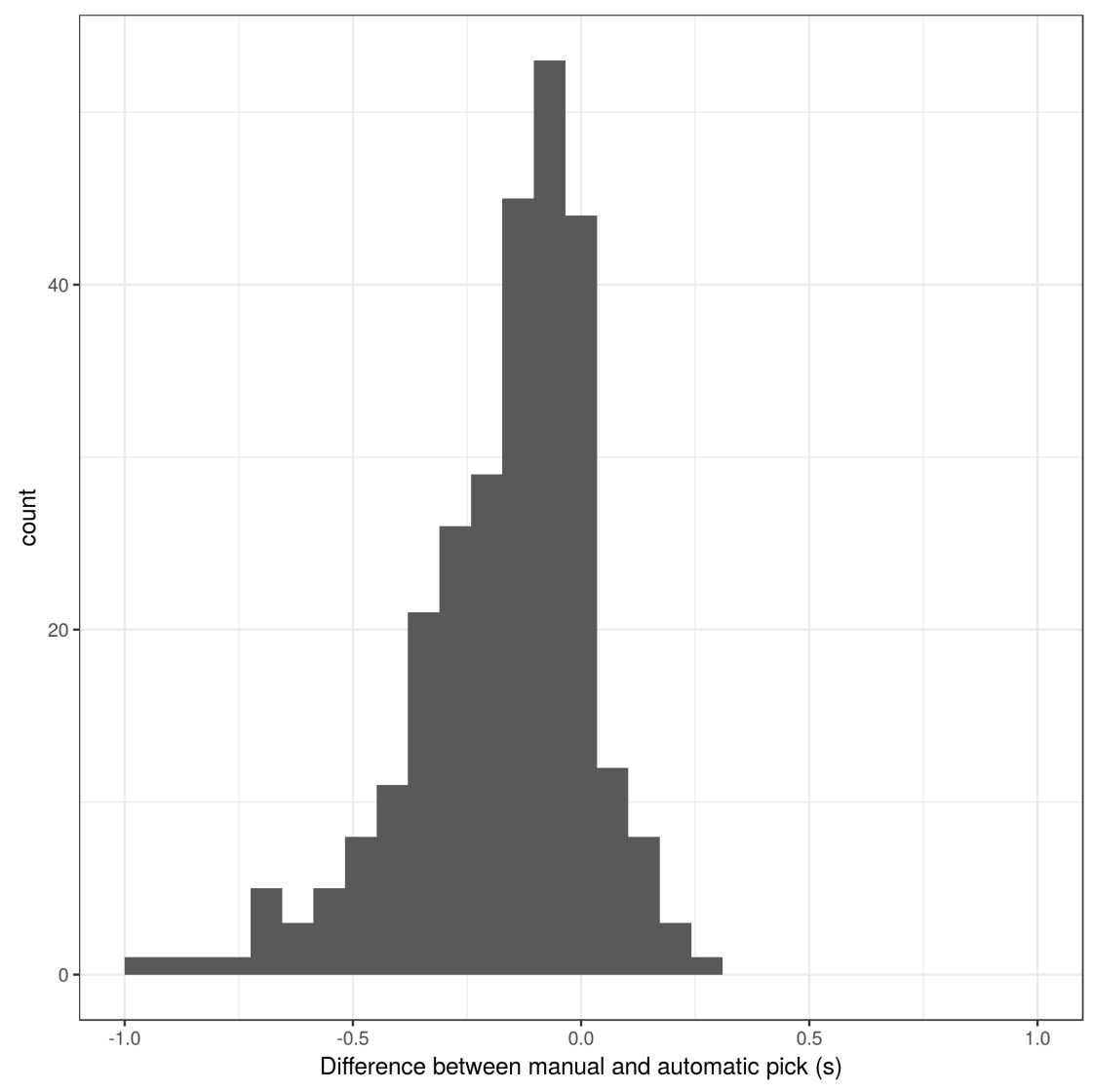

Figure 3.5: Histogram of time difference between manual and automatic picks for events from March to April 2013 from all Rotokawa stations (automatic-manual). The left skewness of the figure is likely a result of the manual operator not always selecting the earliest arrival of the split shear wave. 


\subsection{Automatic shear wave splitting (MFAST)}

To make our shear wave splitting measurements we use the MFAST software package. MFAST is the Multiple Filter Automatic Splitting Technique (Savage et al. 2010a; Teanby et al. 2004; Wessel 2010) available at http://mfast-package.geo.vuw.ac . $\mathrm{nz} /$.

The input into MFAST requires located earthquakes (Section 1.5.2) with their S-wave arrival picked (Section 3.4).

Here we detail MFAST version 2.2 as described in the above references and the MFAST manual (Wessel et al. 2016). The parameters we use are summarised in Table C.2 and largely correspond to the verylocal variation of the MFAST codes (for micro-earthquakes in a small study area with high frequency instruments).

\subsubsection{Filtering}

MFAST filters waveforms before calculating the shear wave splitting. Instead of applying a universal filter, MFAST chooses the best three filters out of a set of filters (summarised in Table 3.2). The best three filters are determined by applying all the filters to each seismogram triplet and determining the signal to noise ratio (SNR) multiplied by the filter bandwidth (in Table 3.2, determined by high_freq/2* low $f r e q)$. The filters with the three highest values for this product are chosen as the best three filters.

All of the best three filters have the shear wave splitting parameters determined. Which of the final three filters chosen as the final measurement for that particular seismogram triplet is detailed in Section 3.6.1. 


\section{Signal to noise ratio}

A SNR is calculated for the filtered data based on two parameters; t_win_snr and $t_{-}$err. In our study these are set to $0.75 \mathrm{~s}$ and $0.02 \mathrm{~s}$ respectively and are used to define two windows. The first noise window, from $t_{-} w i n_{-} s n r+t \_e r r=0.77$ seconds to before the S-wave pick to t_err $=0.02$ seconds before the pick, which includes the 'noise' from the P-wave coda which will affect the S-wave pick. The second signal window is from $t_{-} e r r=0.02$ seconds after the $S$-wave pick to $t_{-} w i n \_s n r+t \_e r r=0.77$ seconds after the pick. The ratio of the root mean square amplitude between the windows is averaged for the north and east components to give a single value of SNR.

\subsubsection{Measuring technique}

\section{Determining shear wave splitting}

The fast polarisation $(\phi)$ and delay time $(\delta t)$ are the parameters which describe shear wave splitting. To make the shear wave splitting measurements and determine these parameters, MFAST uses the method of Silver and Chan (1991) (with corrections from Walsh et al. (2013)). We do not repeat the details here, however they can be found in Walsh et al. (2013).

The motion of an S-wave at the surface can be written in terms of a splitting operator which depends on both $\phi$ and $\delta t(\boldsymbol{\Gamma}(\phi, \delta t))$. This method of determining the shear wave splitting parameters uses a grid search to find the inverse splitting operator $\left(\boldsymbol{\Gamma}^{-1}\right)$ which best undoes the observed splitting on a specified time window (Savage et al. 2010a). The best operator is the one that minimises the second eigenvalue of the covariance matrix of the particle motion. Figure 3.6 shows the contours of the second eigenvalue for a high grade measurement with the $\phi$ and $\delta t$ which minimises it marked. This is equivalent to maximising linearity of the particle motion (Figure 3.7). 
Table 3.2: Table of "verylocal" MFAST filters used in this study. All filters are twopole Butterworth filters. \% indicates the percentage of final high grade shear wave splitting measurements made using that particular filter.

\begin{tabular}{|c|c|c|c|c|}
\hline Filter number & Low freq $(\mathrm{Hz})$ & High Freq $(\mathrm{Hz})$ & Bandwidth (octave) & $\%$ \\
\hline \hline 1 & 1 & 5 & 2.5 & 1.7 \\
\hline 2 & 1 & 8 & 4 & 24.1 \\
\hline 3 & 1 & 15 & 15 & 32.4 \\
\hline 4 & 1 & 30 & 0.83 & 23.7 \\
\hline 5 & 3 & 5 & 2 & 0.6 \\
\hline 6 & 2 & 8 & 2.5 & 0.4 \\
\hline 7 & 3 & 15 & 5 & 0.2 \\
\hline 8 & 3 & 30 & 1 & 13.0 \\
\hline 9 & 5 & 10 & 1.5 & 0.4 \\
\hline 10 & 5 & 15 & 3 & 0.3 \\
\hline 11 & 5 & 30 & 4.5 & 1.1 \\
\hline 12 & 5 & 45 & 1 & 1.2 \\
\hline 13 & 10 & 20 & 2.25 & 0.6 \\
\hline 14 & 10 & 35 & & \\
\hline
\end{tabular}




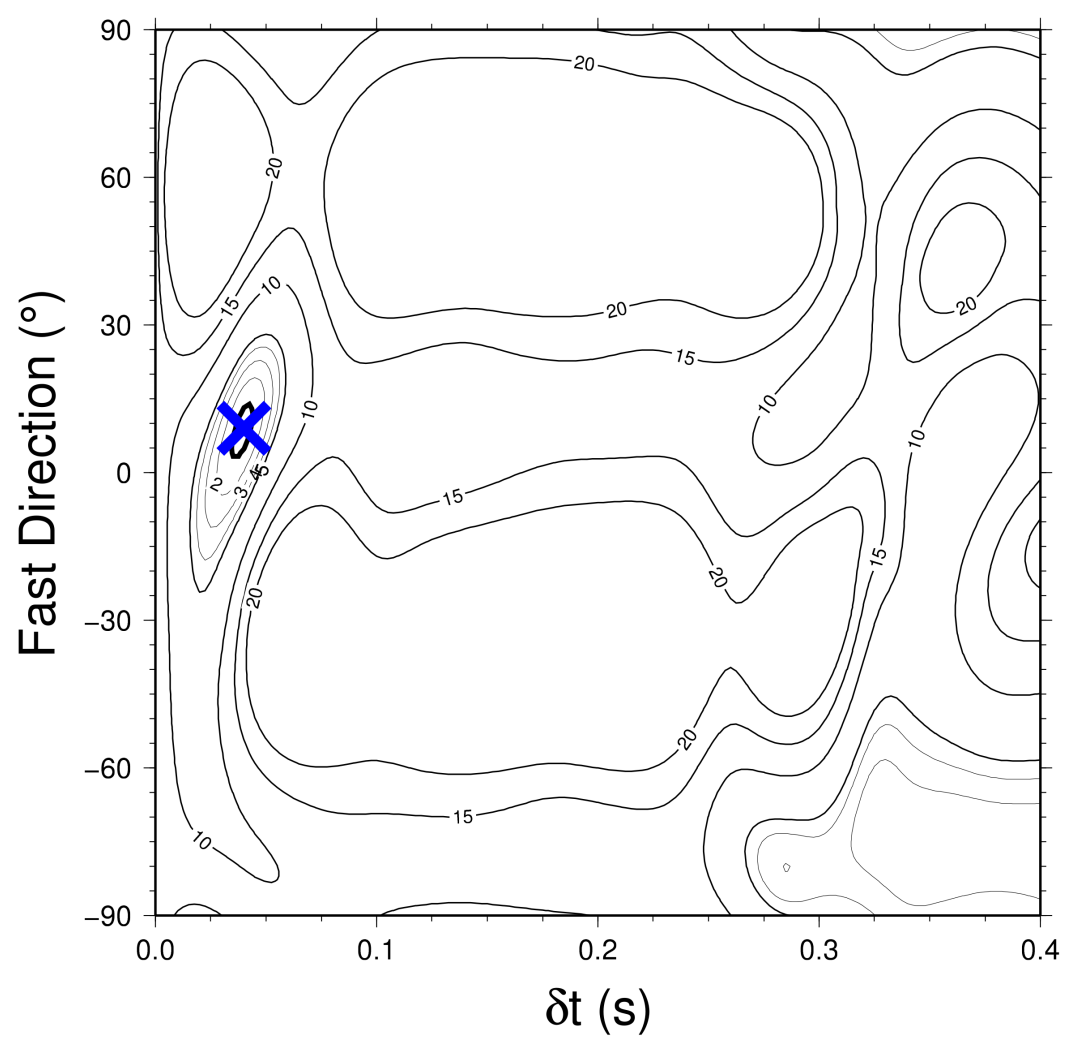

Figure 3.6: Contours of the smallest eigenvalue of the covariance matrix for a high grade measurement. Minimum marked by a blue "X". Fast direction is the same as fast polarisation $(\phi)$. 


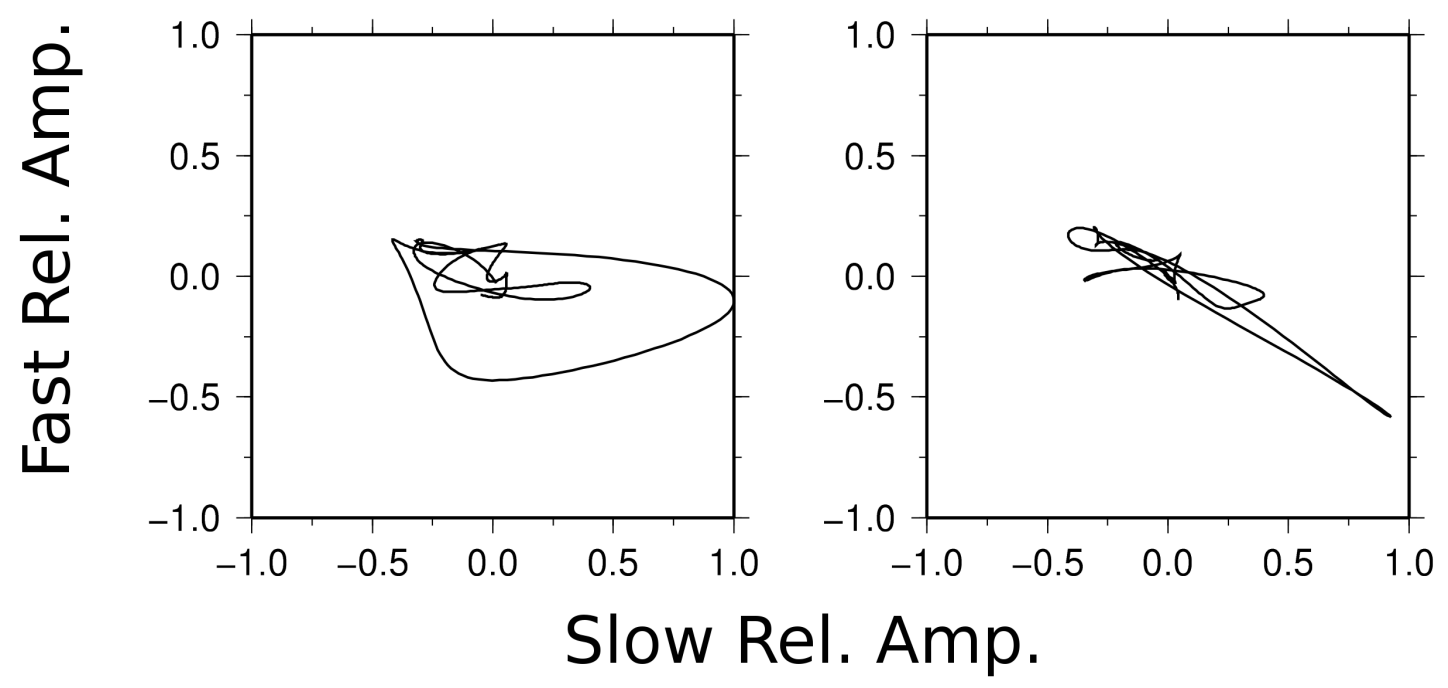

Figure 3.7: Example of particle motion being linearised by the best inverse splitting operator for a high grade shear wave splitting measurement.

\section{Measurement clustering}

The shear wave splitting parameters determined can vary based upon the window chosen to do the measurement. MFAST uses the approach of Teanby et al. (2004), making shear wave splitting measurements on a large number of windows and determining the most stable solution using cluster analyses (Savage et al. 2010a).

The main assumption of Teanby et al. (2004) is that parameters which remain stable over many measurement windows are more likely to be reliable. Firstly, parameters are scaled so that $\phi$ and $\delta t$ (due to their different range) have the same weight. Each window is represented as a point on the scaled $\delta t, \phi$ plane (Figure 3.8). A hierarchical clustering is then applied based on the euclidean distances between points. Each cluster is assigned a variance of the maximum of either its within-cluster variance or its mean data variance. The cluster with the lowest variance is chosen as the best cluster. The best measurement is the measurement from within that cluster 


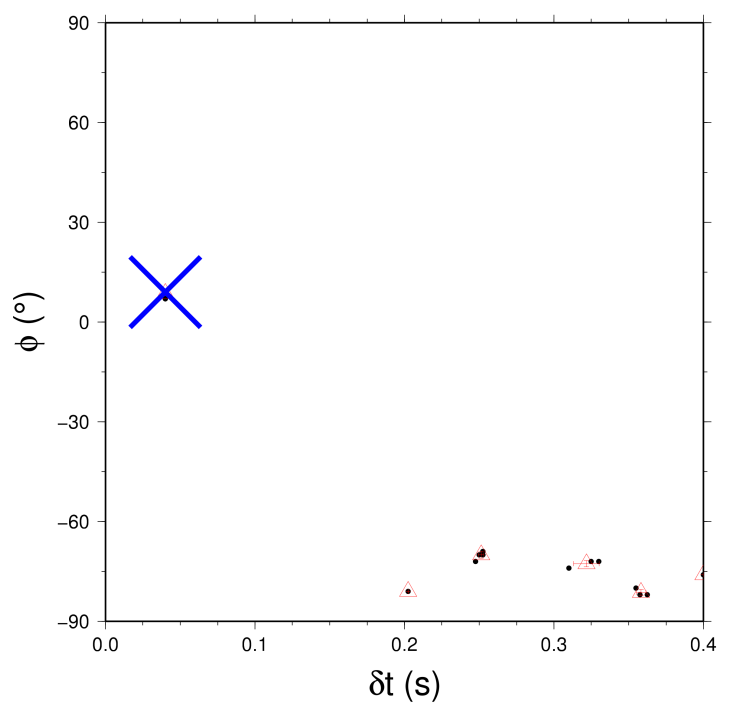

(a) Window clustering

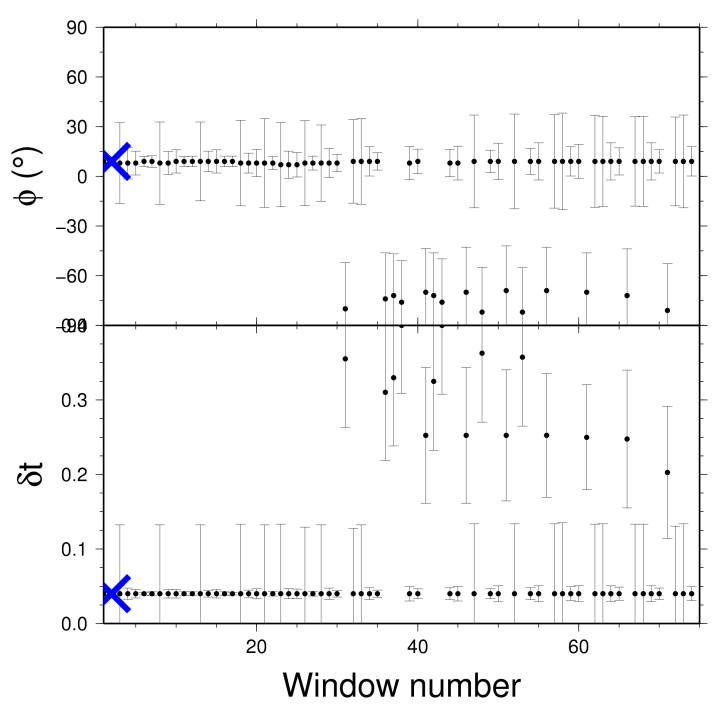

(b) Parameters by window number

Figure 3.8: Clusters of shear wave splitting measurements (a) with the best cluster (containing multiple windows) marked by a blue " $X$ ". (b) shows the parameters by window number with the best measurement marked.

with the smallest variance.

\subsection{Cycle skipping}

Cycle skipping is where waveforms are mismatched by an integer number of half cycles (Savage et al. 2010a). This occurs when the waveform is close to a sinusoid and, due to its repeating nature, mismatching can occur . By selecting the best filter based on the product of SNR and bandwidth (Section 3.5.1,) waveforms with narrow band filters, which are particularly susceptible to cycle skipping, are avoided. Figure 3.9 shows the contours of the smallest eigenvalue for a potentially cycle skipped measurement.

Figure 3.9 shows local minima shifted both by $90^{\circ}$ (where fast and slow polarisa- 
tions are reversed) and repeating minima at regular delay time intervals (where the slow arrival) both of which are evidence for cycle skipping occurring.

Measurements close to the maximum delay time also indicate cycle skipped or noisy data (Savage et al. 2010a) so are also removed (see Section 3.6.1).

\subsubsection{Grading}

\section{Null measurements}

Measurements with $\phi$ in the range of $20^{\circ} \leq|\phi-\Phi p o l| \leq 70^{\circ}$ are graded Null. Apol is the incoming S-wave polarisation and if it is too close to $\phi$ the $\mathrm{S}$-wave may not be split, thus giving an unreliable measurement.

\section{Cluster grading}

MFAST takes all the clusters of measurements, determined using the method of Teanby et al. (2004), and determines a final cluster grade by comparing those with similar variance to the best cluster. If the splitting parameters between the best and a secondary cluster (with comparable variance) are too different the measurement is given a lower cluster grade.

MFAST considers clusters with $\operatorname{var}(k)<5 * \operatorname{var}(k b e s t)$ where $k b e s t$ and $k$ are the best window and $k$ is any other cluster $(\operatorname{var}(x)$ is the variance of $x)$. Grades applied are $\mathrm{ACl}, \mathrm{BCl}, \mathrm{CCl}, \& \mathrm{DCl}$. What constitutes these grades is detailed in Savage et al. (2010a). Grades take into account the difference in variance and differences between $\phi$ and $\delta t$ between clusters. We take only $\mathrm{ACl}$ and $\mathrm{BCl}$ graded measurements.

\section{Parameter grading}

$\mathrm{All} \mathrm{ACl}$ and $\mathrm{BCl}$ measurements (which are not null) are assigned a grade of $\mathrm{AB}$ if their delay time is less than 0.8 times the maximum delay time set in processing. Given 


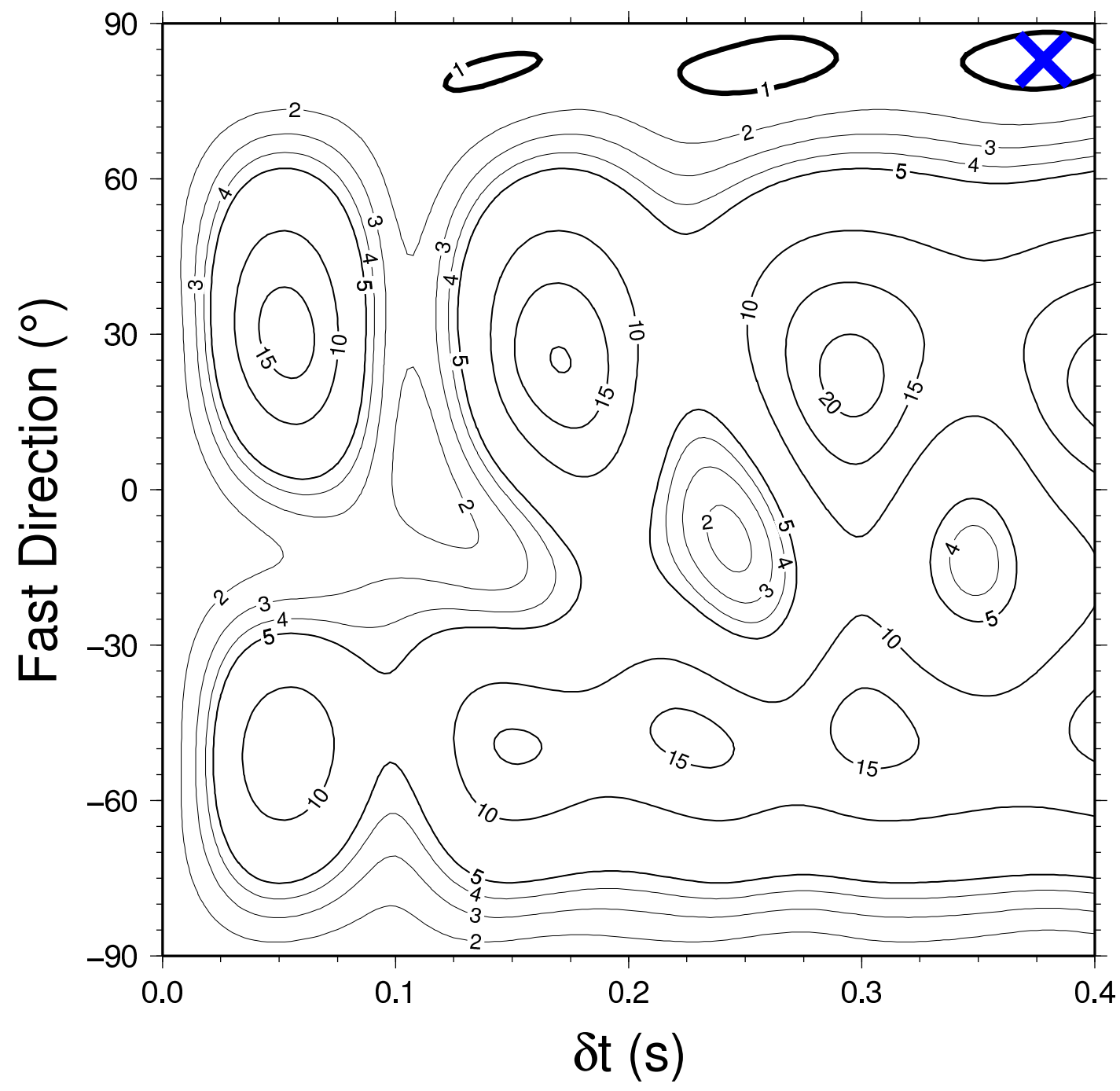

Figure 3.9: Contours of the smallest eigenvalue of the covariance matrix for a measurement which may exhibit cycle skipping. Minimum marked by a blue "X". Fast direction is the same as fast polarisation $(\phi)$. Note the regularly repeating minima which are features of cycles skipping. 
our small study area, we restrict tlagmax (Table C.2) further and only assign AB grades to measurements with $\delta t<0.16$ even though tlagmax, in MFAST processing, is set to 0.4 seconds. $\mathrm{AB}+$ measurements also require an $\mathrm{SNR}>3$ and a error (one standard deviation) of the polarisation to be less than $25^{\circ}$.

\section{Final selection}

We use the same approach as Castellazzi et al. (2015) to select which of the best three filters tested should be selected. We take all AB grade measurements and then assign a second grade based on the similarity of results between the three best filters. For F2 and F3, the filter with the lowest value of error in polarisation is selected.

F1 indicates that only one filter of the seismogram triplet has an $\mathrm{AB}$ measurement.

F2 indicates that the seismogram only has two AB grade measurements and both have polarisations $(\phi)$ within $10^{\circ}$ of each other. There is a subcategory to F2 where there are three $\mathrm{AB}$ grade measurements but only two $\phi$ are within $10^{\circ}$ of each other (F2b).

Finally, F3 indicates that all three filters from that seismogram gave AB grade measurements and all three are within $10^{\circ}$ of each other.

If an seismogram doesn't fall into any of these categories it is discarded (e.g. AB measurements for all three filters but none are within $10^{\circ}$ of each other).

Measurements of either F1, F2/F2b or F3 grades are referred to as "high grade measurements".

\subsubsection{Incoming angle}

The velocity model MFAST uses to calculate incoming angles is a $1 \mathrm{D}$ average of the GNS 3D model for Rotokawa and is quoted in Table 3.3 (Rawlinson 2011 p. 77). Calculations are made by the TauP software (Crotwell et al. 1999). 
Table 3.3: $1 D$ average seismic velocities from the GNS 3D model for Rotokawa (Rawlinson 2011 p. 77). Used for determining angles of incidence and propagation angles.

\begin{tabular}{|l|l|l|}
\hline Depth $(\mathrm{km})$ & $v_{P}(\mathrm{~km} / \mathrm{s})$ & $v_{S}(\mathrm{~km} / \mathrm{s})$ \\
\hline \hline 0 & 2.00 & 1.14 \\
\hline 0.10 & 2.63 & 1.5 \\
\hline 0.60 & 2.8 & 1.61 \\
\hline 1.00 & 2.9 & 1.66 \\
\hline 1.30 & 2.97 & 1.70 \\
\hline 1.60 & 3.05 & 1.74 \\
\hline 1.80 & 3.12 & 1.78 \\
\hline 2.00 & 3.17 & 1.81 \\
\hline 2.20 & 3.2 & 1.83 \\
\hline 2.60 & 3.41 & 1.95 \\
\hline 2.90 & 3.74 & 2.14 \\
\hline 3.40 & 4.32 & 2.47 \\
\hline 4.00 & 4.57 & 2.61 \\
\hline 6.00 & 5.51 & 3.15 \\
\hline 10.00 & 5.81 & 3.32 \\
\hline 20.00 & 6.90 & 3.94 \\
\hline 50.00 & 7.43 & 4.25 \\
\hline & & \\
\hline & & \\
\hline
\end{tabular}




\subsection{Earthquake path clustering}

Clustering earthquakes by their location is useful as it provides an indication of where measured anisotropy originates. When investigating temporal changes it also allows migration of the seismicity to be ruled out as a cause of changing anisotropy.

Seismicity is generally confined to a single area in Rotokawa and two distinct areas in Ngatamariki. Clustering these earthquakes purely by location proved inadequate for three reasons. Firstly, to be able to recover statistically significant means the clusters had to contain a sufficient number of measurements. Secondly, doing a simple clustering with k-means gave clusters which contained all events from a particular field, thus the assumption that events in a clusters have a similar location no longer holds. Finally, given that shear wave splitting is measured not at a point but rather along the station-event path, it is more intuitive to cluster the station-event paths so that events with similar paths to the same station group together.

In proceedings, we have published data where station-event path clustering is the final processing step applied to that data (Mroczek et al. (2016), Appendix F). In this thesis, we instead treat this as an intermediate step by fitting these station-event path clusters to theoretical crack planes (as described in Section 3.8).

\subsection{1 movMF}

The von Mises distribution (the circular equivalent to a normal distribution, Section 3.2.2) generalised to three (or more dimensions) is known as the von Mises-Fisher distribution and is thus common place in spherical statistics. In our approach for clustering our station-event paths we use the movMF package (Hornik and Grün 2014), in R (Section E.1), to fit mixtures of von Mises-Fisher distributions to a unit hemisphere.

Using finite mixtures of von Mises-Fisher distributions to cluster on a unit hemi- 
sphere was introduced by Banerjee et al. (2005). The methods behind the clustering process is complex and is outlined by Hornik and Grün (2014), which we will summarise here. We use the soft-movMF expectation maximisation (EM) algorithm (Banerjee et al. 2005).

The (soft) expectation maximisation algorithm has two major steps, a expectation step which calculates a-posteriori probabilities (i.e. estimating the full dataset using the current estimate of the parameters), and a maximisation step, where the complete-data likelihood estimate for the parameters is determined (Hornik and Grün 2014; Moon 1996). These steps are repeated in an iterative process until there is convergence or (in our case) the algorithm has repeated $n$ times. We choose to iterate the algorithm 20 times rather than test for convergence so that the algorithm doesn't get stuck in a local minimum (20 iterations is enough to reach a solution which converges). Each data point is then clustered by choosing the von Mises-Fisher distribution which gives maximum a-posteriori probabilities (i.e. which cluster they are most likely to fall into).

The EM algorithm has to be run with the number of components (number of final clusters) selected beforehand. Since the number of clusters that are present is unknown then, similarly to Hornik and Grün (2014), we use the Bayesian information criterion (BIC). The BIC is a measure of fit of a model to data that penalises for adding more components (to prevent over fitting) (e.g. McLachlan and Peel 2004). We repeat our clustering with 1-7 components and select the number of components with the lowest BIC as our final clustering. BIC functionality is included in $\mathrm{R}$ ( $\mathrm{R}$ Core Team 2013).

\subsubsection{Processing}

We do not directly cluster station-event paths. Instead we approximate them by calculating the pierce points of the straight line station-event paths on a unit hemi- 


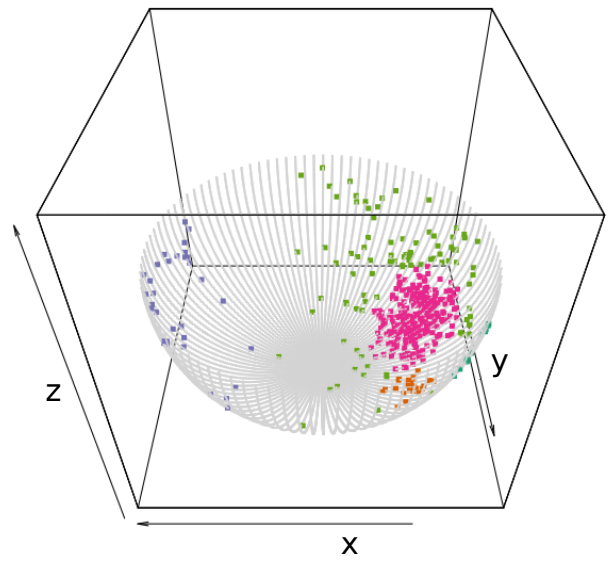

(a) Pierce points

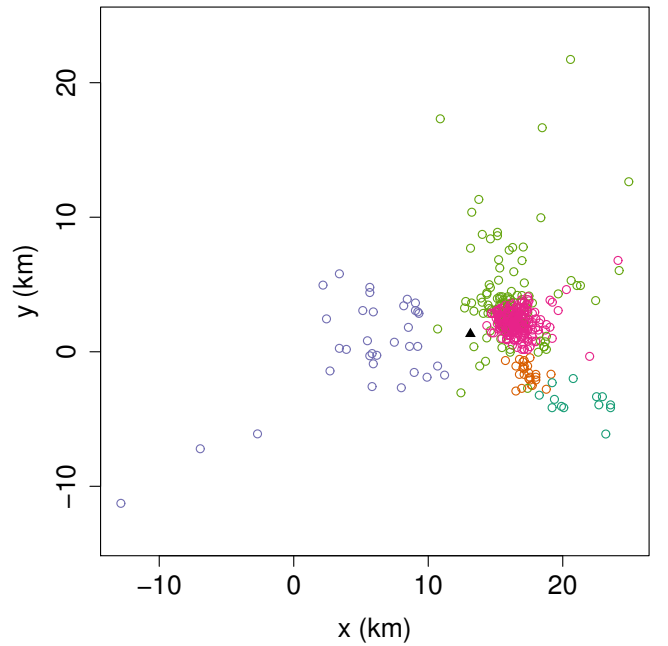

(b) Map view of event locations

Figure 3.10: Clustered pierce points of station RT01 shown on a unit hemisphere below the station.

sphere below each station (Figure 3.10). As these pierce points are on a (hemi)sphere they can be clustered using movMF. A pierce point is the location where a path intersects a surface. Only stations with more than 55 measurements are clustered using this method as sufficiently large samples are required. Mixtures of von Mises-Fisher distributions (the spherical equivalent of a normal distribution) are then fit to the pierce points using the movMF package (Hornik and Grün 2014) detailed in Section 3.7.1.

Each of these clusters are then fitted to a theoretical crack plane (Section 3.8). Previously we removed clusters which failed the Rayleigh test (Mroczek et al. (2016), Appendix F). However, due to the underlying distribution of polarisations not being unimodal, certain clusters may fail the Rayleigh test while still having a good fit to a theoretical crack plane so we include them. 


\subsection{Crack fitting with MSAT}

The Matlab Seismic Anisotropy Toolkit (MSAT) is a toolkit for the study and analysis of seismic and elastic anisotropy (Walker and Wookey 2012). We take advantage of several functions from the toolkit to calculate misfit, model data and plot results. The package is available at http://www1.gly.bris.ac.uk/MSAT.

The MSAT functions which we use to achieve the crack fitting are described in Appendix D.

\subsubsection{Crack model}

The fitting process requires an elasticity matrix of the anisotropic material to be defined. We use the model of Hudson $(1980,1981)$ for a cracked solid. This model determines the elastic parameters which define the motion of a wave through a material (to second order) with scattering inclusions within it. Hudson's model can be used to formulate the elasticity tensor for a material for thin aligned cracks. This requires several parameters to be defined which we input into the function MS_hudson_cracks (described in Section D.1). Since we are fitting orientations $(\phi)$ and relative time delays, most of these parameters can be arbitrary selected as they only affect the absolute values of $\delta t$. However we do attempt to select reasonable values (Table 3.4). We define the values of the fill material as those for water as we assume the majority of our cracks are saturated.

The values for the matrix parameters are chosen more arbitrarily as they do not affect polarisations. Absolute values of percent anisotropy are affected by $v_{P} / v_{S}$ ratio of the matrix so we select one close to a Poisson solid $\left(v_{P} / v_{S}=\sim 1.7\right)$. Crack density

defined as $c d=\frac{N a^{3}}{v}$ where $N$ is the number of "penny" shaped cracks of radius $a$ in a volume $v$. A crack density of 0.05 is roughly the upper limit of what you would expect for an intact rock (e.g. Crampin 1999). We assume crack aspect ratio is 
Table 3.4: Parameters for the crack model of Hudson (1980, 1981).

\begin{tabular}{|l|l|l|}
\hline Variable & Value & Description \\
\hline \hline$v_{p}(m)$ & $6.3 \mathrm{~km} / \mathrm{s}$ & Isotropic P-wave velocity of matrix \\
\hline$v_{s}(m)$ & $3.63 \mathrm{~km} / \mathrm{s}$ & Isotropic S-wave velocity of matrix \\
\hline$\rho_{m}$ & $2800 \mathrm{~kg} / \mathrm{m}^{3}$ & Isotropic density of matrix \\
\hline$v_{p}(c)$ & $1.483 \mathrm{~km} / \mathrm{s}$ & Isotropic P-wave velocity of fill material \\
\hline$v_{s}(c)$ & $0 \mathrm{~km} / \mathrm{s}$ & Isotropic S-wave velocity of fill material \\
\hline$\rho_{c}$ & $1000 \mathrm{~kg} / \mathrm{m}^{3}$ & Isotropic density of fill material \\
\hline ar & 0.0001 & Aspect ratio of cracks \\
\hline cd & 0.05 & Crack density \\
\hline
\end{tabular}

negligible (0.0001) (Peacock et al. 1988). Varying (over realistic values of); crack density, densities (for crack and fill materials), and velocities does not significantly affect polarisations.

\subsubsection{Fitting process}

To fit theoretical crack planes we take the raw splitting data from MFAST and compare it to synthetic splitting parameters $\left(\phi_{\text {synthetic }}, \delta t_{\text {synthetic }}\right)$ calculated with MS_phaselevels (Section D.3). The final misfit is averaged between the misfit between $\phi$ and the misfit for $\delta t$. Polarisation misfit $\left(\phi_{\text {misfit }}\right)$, where unwind $(x)$ indicates $x$ is moved onto the range of $-90^{\circ}$ to $90^{\circ}$ by adding or subtracting a multiple of $180^{\circ}$, is determined by:

$$
\text { misfit }_{\phi}=\frac{\sum_{n=1}^{N}\left(\text { unwind }\left(\left|\phi_{n}-\phi_{n, \text { synthetic }}\right|\right) / 90^{\circ}\right)}{N}
$$

for $N$ polarisations.

MS_phaselevels outputs the fast and slow S-wave velocities $\left(v_{S 1} \& v_{S 2}\right)$ so 
synthetic delay times can be determined by

$$
\delta t_{\text {synthetic }}=d / v_{S 2}-d / v_{S 1}
$$

These synthetic delay times depends on distance travelled through the anisotropic medium $(d)$ which we select arbitrarily, as well as properties of the cracked medium being modelled, namely crack density. As a result, there is no guarantee that synthetic time delays will be close to the actual delay times. So, instead of applying other fitting techniques which require precise synthetic time delays, we correlate the synthetic time delays with the actual time delays. So, rather than fitting absolute time delays, we're fitting the pattern of time delays. We rescale the correlation to be on the same range as misfit $t_{\phi}(0$ to 1 ) and convert it to a distance (for $N$ delay times).

$$
\operatorname{misfit}_{\delta t}=1-\frac{\operatorname{corr}\left(\delta t, \delta t_{\text {synthetic }}\right)+1}{2}
$$

Final misfit is the mean of the individual misfits

$$
\text { misfit }=\frac{\text { misfit }_{\phi}+\text { misfit }_{\delta t}}{2}
$$

misfit is determined at each degree of dip and strike by utilising MS_rot3 (Section D.2) to rotate the elasticity matrix.

The output of this fitting process is a table of misfits calculated at each value of strike and dip. This grid gives the best fitting strike and dip and allows as comparison between misfit groups from different stations or groups of shear wave splitting measurements.

\section{First pass of clustered measurements}

We take the path clustered shear wave splitting measurements (described in section 3.7) and run the fitting algorithm on each of them. For the first pass of clustering the misfit is calculated every $5^{\circ}$ of strike and every $2.5^{\circ}$ of dip. Figure 3.11 shows one of the path clusters from station RT05 and its best fitting pole figure. 


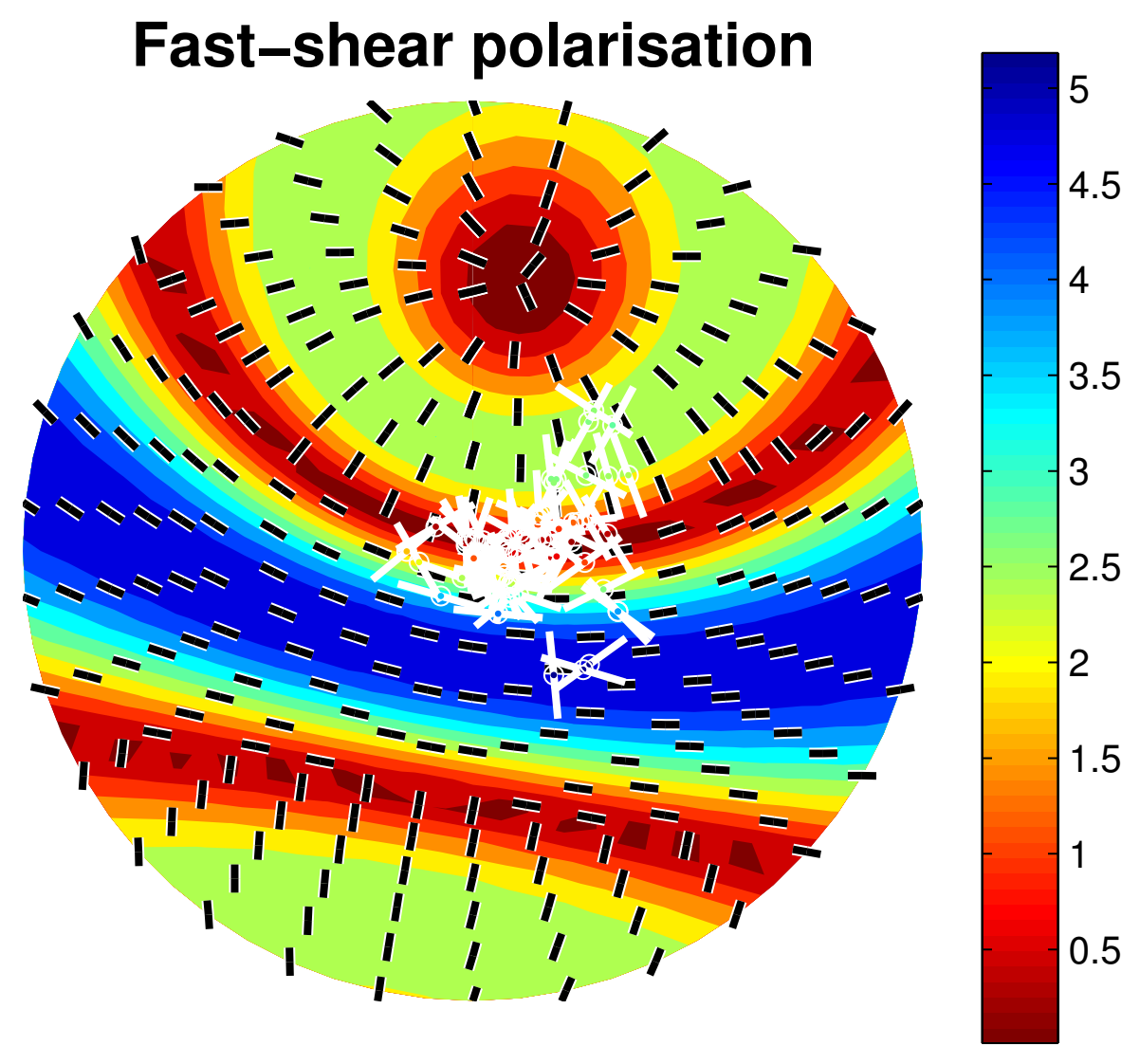

Figure 3.11: Theoretical S-wave polarisations for best fitting crack plane with RT05 cluster shown on lower hemisphere angle preserving pole figure. Overlaid with actual polarisations from the cluster shown in white. Black lines represent the theoretical polarisations and color contours show percent anisotropy. 


\section{Hierarchical clustering of misfits}

To group together earthquake path clusters with similar misfit grids we apply a hierarchical clustering method. To avoid confusion the resulting clusters from this method will be referred to as groups.

After the first pass of fitting orientation, the output misfit grids are correlated

with each other. The correlation is completed using R's cor() function (described in section 3.2.5). This produces a table of correlation coefficients between different earthquake path clusters. Hierarchical clustering requires a table of distances rather than correlations. To perform this conversion we use distance $=1-$ correlation. This means that misfit grids with negative correlation have the highest distance. With minor changes to the table of distances, we perform a hierarchical clustering (described in Section 3.2.8).

Shear wave splitting measurements made on the same station with different station-event paths, provided they are sampling from the same crack plane, will have similar misfit grids and thus hierarchical clustering will group them back together.

\section{Second pass of grouped measurements}

We take the result of the hierarchical clustering and group them with a cut off distance of 0.5 (equivalent to a correlation coefficient of 0.5 ). We then re-run the fitting process for these groups. For the first pass of clustering the misfit is calculated every $5^{\circ}$ of strike and every $2.5^{\circ}$ of dip.

\section{Third pass of grouped measurements}

It was observed that several groups had high correlation with each other after the second pass of hierarchical clustering and fitting. For the third pass we use a stricter cut off distance of 0.3 (equivalent to a correlation coefficient of 0.7 ) and then re-run the fitting process. 
The outputted groups of this third pass are the final groups which are then graded.

\section{Grading}

Rather than comparing groups using absolute misfit, we use a second method to measure the uniqueness of a particular best fit by comparing the misfit of the best fitting crack plane with the other local minima for that group of measurements. It is generally the case that a group with high uniqueness will also have low absolute misfit.

Firstly, we remove any groups with less than fifty measurements to remove outlying groups with high error.

Secondly, to determine how unique the fit is, we calculate the difference between the global minimum (best fitting strike and dip) and the misfit of the next best local minima (second lowest misfit). The higher this difference the more unique the global minimum is compared to the local minima and thus the fitting gives a more unique solution.

Local minima are determined using a grid search. If the misfit of cells adjacent to the cell in question are all higher, then that cell is a local minimum. Edge effects cause adjacent cells to have the same (minimum) misfit, which we account for by only considering unique misfits to be minima (i.e. if the minimum is spread across three cells then the algorithm accurately records it as a single minimum). Figure 3.12 shows a comparison between misfits grid with low and high uniqueness.

\subsubsection{Angle of incidence versus angle of propagation}

We approximate the propagation angle through the cracked medium with the angle of incidence at the surface (Section 3.6.2). The velocity model is defined up to sea level with the final layer being $100 \mathrm{~m}$ thick. Thus, the propagation angle we use for our crack fitting process is in fact the estimated angle in this final layer. The 


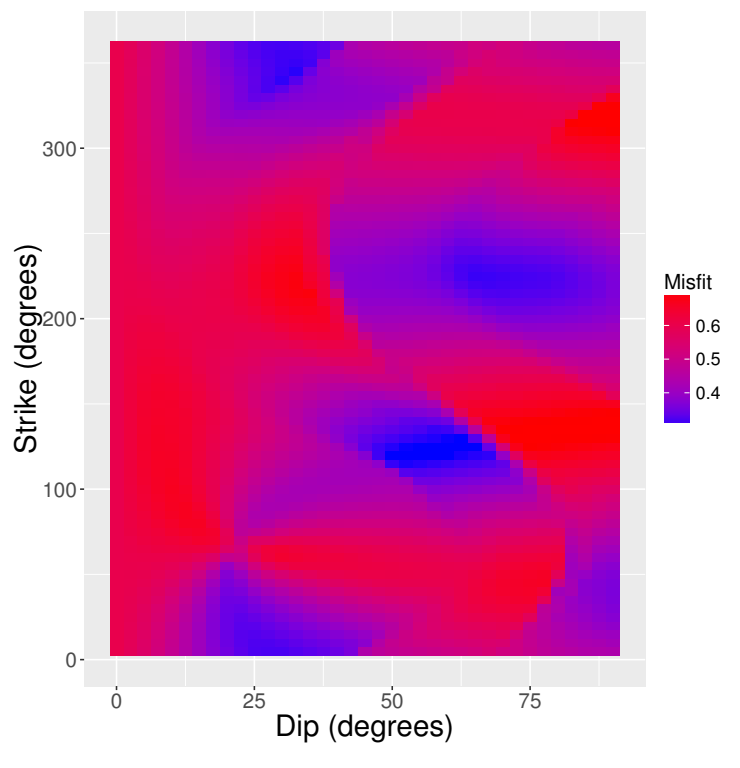

(a) Low uniqueness

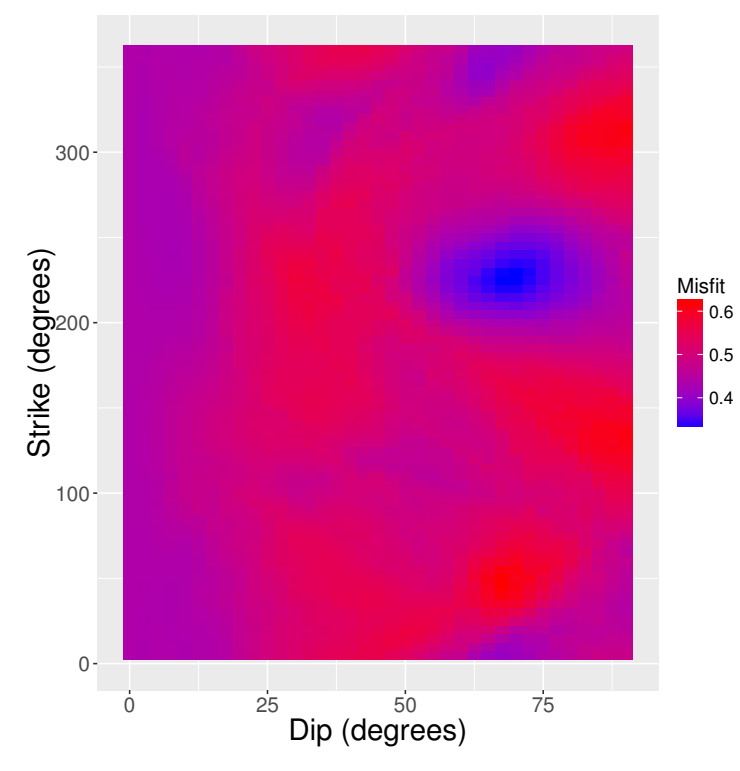

(b) High uniqueness

Figure 3.12: Misfit grids for two groups of shear wave splitting measurements. Blue indicates low misfit.

shear wave splitting we measure is thought to be located closer to the station on the station-event path (Rümpker and Silver 2000; Nistala and McMechan 2005) and there is some evidence for this in our dataset (Section 4.3.2). Whereas under the assumption of a single homogeneous anisotropic body, from which we are sampling shear wave splitting measurements, the angle of propagation would be constant.

Thus, we have two end members; straight line propagation angle and propagation angle in the final layer. The mean difference between the two is $\sim 25^{\circ}$ across all our stations. We run the crack fitting process for both a uniform velocity model and for our more accurate Rotokawa model. In our results, we focus fitting measurements whose angle of propagation is estimated from the Rotokawa velocity model.

Working under the assumption that cracks are near vertical and strike does not vary with depth, a wave that begins propagating in the anisotropic layer at a subvertical angle will remain sub-vertical and thus the polarisation will not change. 
Incoming waves which begin at shallower propagation angle but, due to lower velocity layers, enter the sub-vertical range (assuming crack orientation remains constant) will have their polarisation switched. The previously slow polarisation will become the fast polarisation and the previously fast polarisation will become the slow polarisation. If the new fast polarisation (formerly the slow polarisation) catches up with the old fast polarisation and overtakes it we will measure the correct crack strike. If it does not we will measure a polarisation $90^{\circ}$ to the crack strike. For both these cases we would expect anomalously low delay time per kilometre $(\delta t p k m)$. We find evidence for this in our dataset. Events with angles of incidence less than 35 degrees (with the Rotokawa velocity model, see Section 3.6.2) but with straight line angles of incidence greater than $45^{\circ}$ have a mean $\delta t p k m$ of $0.012 \mathrm{~s}$ while events with straight line angles of incidence less than $35^{\circ}$ have mean $\delta t p k m$ of $0.066 \mathrm{~s}$, almost six times larger.

\subsubsection{Applications of restricting measurements}

By determining the best fitting theoretical crack plane for each group, we can restrict our dataset of shear wave splitting measurements to only those that have propagation angles such that their fast polarisations are theoretically parallel to the crack plane and their delay times do not vary with propagation angle.

We achieve this by, for each real shear wave splitting measurement (Figure 3.13a), taking the propagation angle of the actual measurement and using the best fitting crack plane to predict what the fast polarisation and shear wave velocities would be at that prorogation angle (Figure 3.13b). Thus, for an arbitrary travel distance of $2 \mathrm{~km}$, we can predict what the percent anisotropy would be for each measurement. We then restrict our dataset to just those real shear wave splitting measurements that have a predicted percent anisotropy $\geq 4 \%$. So only shear wave splitting measurements that, based on their propagation angle, are theoretically polarised in the same direction as 


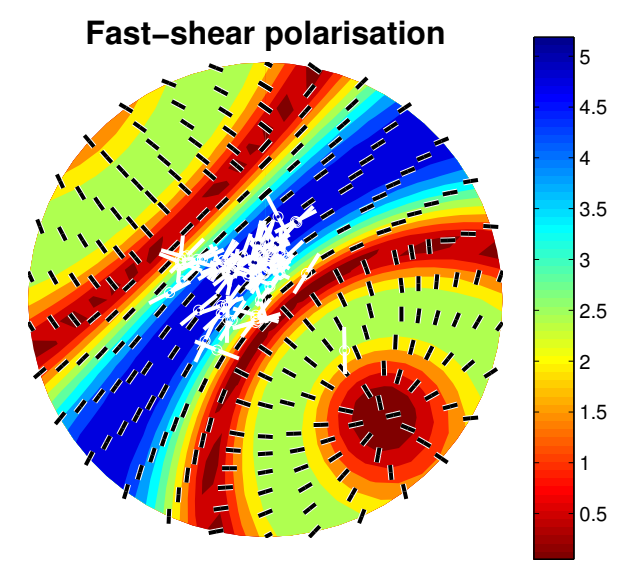

(a) Actual measurements

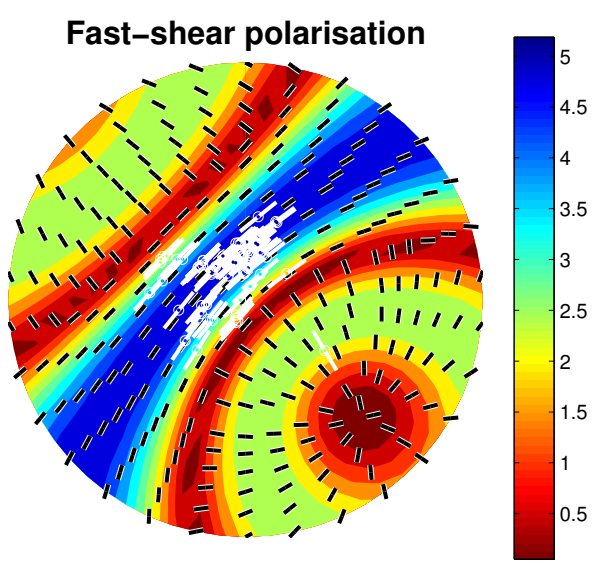

(b) Modelled measurements

Figure 3.13: Best fitting lower hemisphere overlaid with real shear wave splitting measurements (a) and expected polarisations for those measurements (b).

their crack plane and have uniform delay time make up the restricted dataset. For example, in Figure 3.13, measurements which fall into the dark blue contour make up the restricted dataset. The modelled polarisations and velocities are only used to determine which actual measurements have a theoretical percent anisotropy $\geq 4 \%$ and not for any processing.

Additionally, this central region (that we restrict to) is stable over many crack parameters while the outer contours are more susceptible to change.

\subsection{Tomography estimation and spatial averaging (TESSA)}

Spatial averaging of shear wave splitting fast polarisations and delay time tomography give a quick and generally good way of digesting thousands of measurements at once to understand the average trends of the dataset. For this approach we use 
the TESSA (Tomography Estimation and Shear-wave-splitting Spatial Average) package (Johnson 2011). In this section we summarise Johnson (2011) and Johnson et al. (2011). The package is available at https://sites.google.com/site/ jessicahelenjohnson/tessa.

Johnson (2011) and Johnson and Savage (2012) remove measurements with an angle of incidence greater than $35^{\circ}$ due to shear wave window considerations before using TESSA. Both the 2-D spatial averaging and the delay time tomography work on the assumption that shear wave splitting fast polarisations are parallel to their corresponding crack orientations (i.e. propagation angles are sub-vertical). The filtering out of measurements with high angles of incidence (the approach of Johnson et al. (2011)), for the most part, acts to restrict the dataset to sub-vertical propagation angles. However $35^{\circ}$ cut off is often unnecessarily harsh, for a uniform velocity model, due to low velocity layers in the near surface (Section 5.1). A more elegant solution is to instead restrict the inputs for TESSA using the fitted crack planes (described in Section 3.8.4). This way we can restrict our dataset input into TESSA to those measurements which theoretically should be have fast polarisations parallel to their best fitting crack plane and have delay times independent of propagation angle.

\subsubsection{Dataset restriction}

TESSA works on the assumption that angles of propagation are sub-perpendicular to the axis of symmetry of the anisotropy, allowing measurements to be averaged in a simple way. By restricting the dataset, as described in Section 3.8.4, we can remove measurements (often at shallow propagation angles) that may have $\phi$ flipped up to $90^{\circ}$ and have reduced delay times. How velocities depend on propagation angle is described in Section 2.2.2. The measurements in the restricted dataset are all real shear wave splitting measurements. 
Our fitting method does not account for absolute time delays so the percentages of anisotropy shown in Figure 3.13b are purely used to define the region where the delay times/percent anisotropy are invariant with incoming angle and back azimuth. For cracks with a large amount of dip, it is possible that some measurements which fall into this blue area are not parallel with the strike of the cracks thus would be incorrect for the 2-D spatial averaging, however inspection of the pole figures (Appendix B) shows that there are no measurements which fall into this region for any highly dipping crack planes.

\subsubsection{Quadtree gridding}

We grid our study area using the quad-tree method (Townend and Zoback 2001) (Figure 4.30a). This gridding is used for both spatial averaging and tomography. Due to our much smaller study area our parameters differ from those used by Johnson et al. (2011). To reflect our study area being very roughly a 5th of the size we adjust most parameters by a factor of 5 . Original parameters can be found in Johnson et al. (2011) and our parameters are summarised in Table 3.5.

The gridding is performed with respect to the maximum and minimum allowable number of rays per box as well as the minimum box size (Table 3.5). The quad-tree algorithm works in an iterative process. At each iteration the number of raypaths in a block is counted and if it is above the maximum the block is divided further and if it is below the minimum the block is excluded (Johnson 2011, Chapter 4). This process continues until either the number of rays falls between the minimum and maximum or the block size reaches the minimum allowed (Johnson 2011, Chapter $4)$. 


\subsubsection{Tomography estimation}

Delay time tomography utilises the same quad-tree grid used for the 2-D spatial averaging (described in section 3.9.4). It relies on the assumption that the delay time that is accumulated along the raypath is equal to a sum along the entire raypath (Johnson et al. 2011).

Thus the delay time measured at the station for the $r_{t h}$ event $\left(d t_{r}\right)$ is a function of the delay time per kilometre of the $b_{t h}$ block $\left(s_{b}\right)$ and the length of the raypath in that block $\left(L_{r b}\right)$ summed across the $n$ blocks which the raypath traverses.

$$
\delta t_{r}=\sum_{b=1}^{n} s_{b} * L_{r b}
$$

which allows the inverse problem to be set up and anisotropy of each box of the grid to be inverted for, details of which can be found in Johnson et al. (2011).

\subsubsection{Spatial averaging}

Once the quad-tree grid has been been completed, rays which pass through each box are weighted and then are averaged using the correct considerations for axial quantities (Section 3.2.1) and plotted. We weight measurements by distance along raypath by $1 / d^{2}$ as $\phi$ will be affected more by anisotropy later in its path (Rümpker and Silver 2000; Nistala and McMechan 2005). This appeared to be the best weighting scheme tested by Johnson et al. (2011). 
Table 3.5: Processing parameters for delay time tomography and polarisation spatial averaging (TESSA)

\begin{tabular}{|l|l|l|}
\hline Parameter & Value & Description \\
\hline \hline minsizeCB $(m)$ & 1000 & Minimum box size for checkerboard test \\
\hline bin $(m)$ & 200 & Node spacing \\
\hline minsize $(m)$ & 300 & Minimum box size \\
\hline maxnumber & 325 & Maximum number of rays allowed in each box \\
\hline minnumber & 50 & Minimum number of rays allowed in each box \\
\hline weight_p & 3 & Sets to $1 / d^{2}$ weighting \\
\hline grid & 0 & Sets to quadtree gridding \\
\hline
\end{tabular}

\subsection{0 $v_{P} / v_{S}$ ratios}

The ratio of p-wave velocity $\left(v_{P}\right)$ to $\mathrm{S}$-wave velocity $\left(v_{S}\right)$ is equivalent to the ratio of S-wave travel time $\left(t_{S}\right)$ to $\mathrm{P}$-wave travel time $\left(t_{P}\right)$ when assuming the velocities are an average along the path of the seismic waves (Nur 1972). This can be easily shown:

$$
\text { Velocity }_{\text {average }}=\text { distance/time }
$$

thus

$$
v_{P} / v_{S}=\frac{d / t_{P}}{d / t_{S}}
$$

which simplifies to

$$
v_{P} / v_{S}=t_{S} / t_{P}
$$

All the catalogue and matched filter events we use in shear wave splitting have Pwave and $\mathrm{S}$-wave arrival times picked so $t_{P}$ and $t_{S}$ are already known and therefore calculation of $v_{P} / v_{S}$ is trivial using the formula above. 


\section{Chapter 4}

\section{Results and discussion}

\subsection{Station orientation}

Shear wave splitting requires that the stations be oriented correctly. For an unorientated station the shear wave splitting fast polarisations will have an unknown fixed offset equal to the mis-orientation of that station. We determined orientations for the three borehole stations in our network (NS12, NS13, and NS14) using the Rayleigh wave method (Section 3.3). The orientation of the North components for each of the stations is summarised in Table 4.1.

The orientations determined give the corrections which need to be applied to shear wave splitting fast polarisations. The correction is applied immediately after MFAST processing and is determined by:

$$
\phi_{\text {corrected }}=\phi+\text { orientation }
$$

$\phi_{\text {corrected }}$ is then "unwound", so that it falls on the range of $-90^{\circ}$ to $90^{\circ}$, by adding or subtracting multiples of $180^{\circ}$.

Stations orientations, given in Table 4.1, are plotted in Figures 4.1, 4.2, and 4.3. Estimates are calculated using only "good" measurements (see Section 3.3) and give 
Table 4.1: Borehole station orientations (azimuth of the North component). S.D. and S.E. are standard deviation and standard error respectively. Mean and standard deviation/error are calculated from only "good" measurements.

\begin{tabular}{|c|c|c|c|c|c|c|c|}
\hline Station & Orientation $\left(^{\circ}\right)$ & S.D. $\left(^{\circ}\right)$ & S.E. $\left(^{\circ}\right)$ & Good & Quest. & Bad & Total \\
\hline \hline NS12 & 285.73 & 13.27 & 3.9 & 15 & 6 & 148 & 169 \\
\hline NS13 & 292.03 & 14.69 & 2.8 & 32 & 18 & 112 & 162 \\
\hline NS14 & 65.31 & 9.35 & 4.5 & 5 & 5 & 159 & 169 \\
\hline
\end{tabular}

the estimated azimuth of the North component.

Shear wave splitting measurements for NS13 (once corrected for station orientation) match closely with the expected NE-SW fast polarisation orientation in Ngatamariki with $\phi_{\text {mean }}$ of $055^{\circ}$.

\subsubsection{Errors in orientation}

We calculate a standard error for each of the estimated orientations (using the same method as with other standard error calculations but without doubling the angles, see Section E.2.9).

Standard error gives an estimate of how much the calculated orientation may differ from the true orientation of the station and reflects how accurate the measurement is. For example, NS14 has a lower standard deviation than the other two stations however, (with only five measurements) it's standard error is higher (Table 4.1).

Standard errors for all stations are of the same order as the error of the individual fast polarisations and well within the standard error of the mean for a years worth of data on a individual station. Thus, we make the correction to the measurements made at borehole stations and then treat them in the same manner as other stations. 


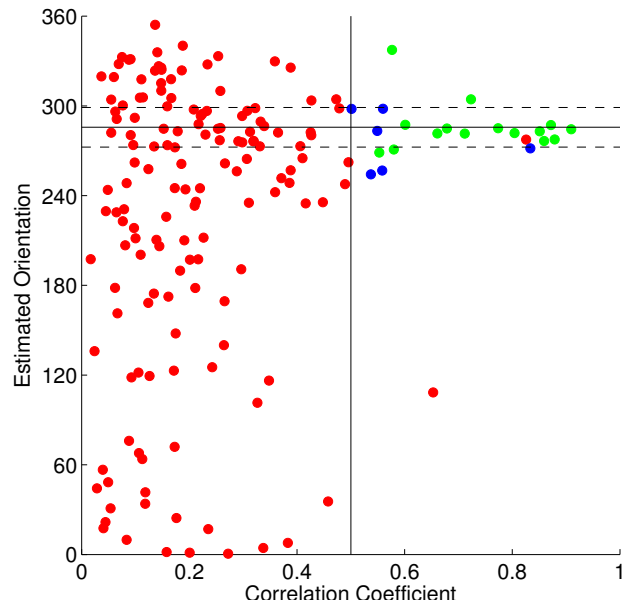

(a) Correlation versus orientation

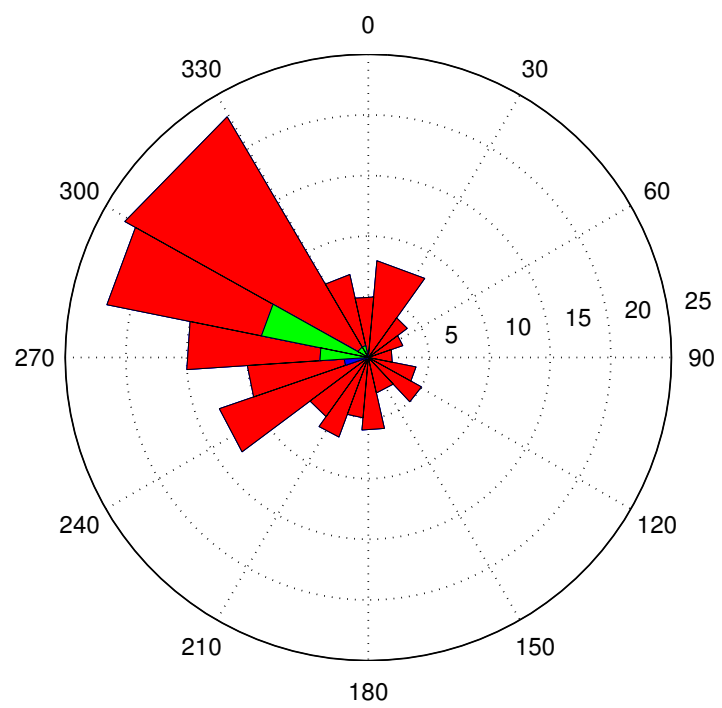

(c) Rose diagram of orientations

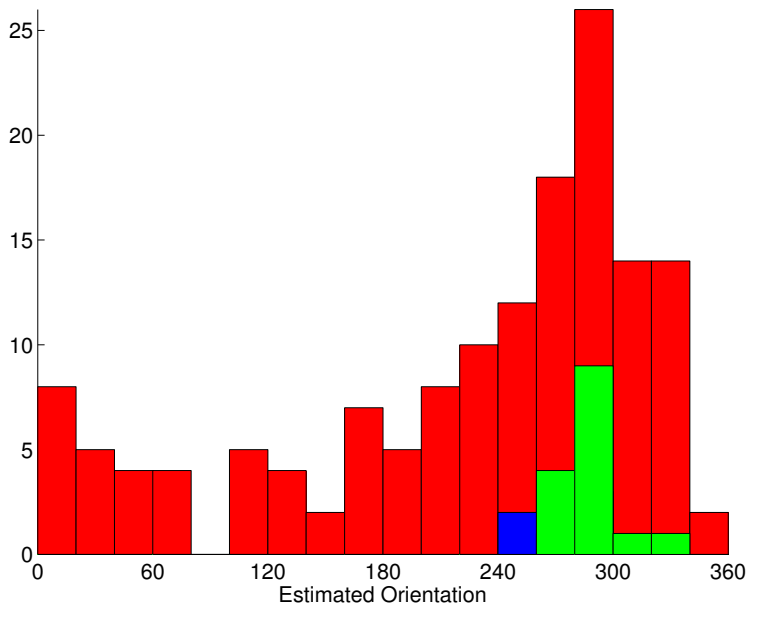

(b) Histogram of orientations

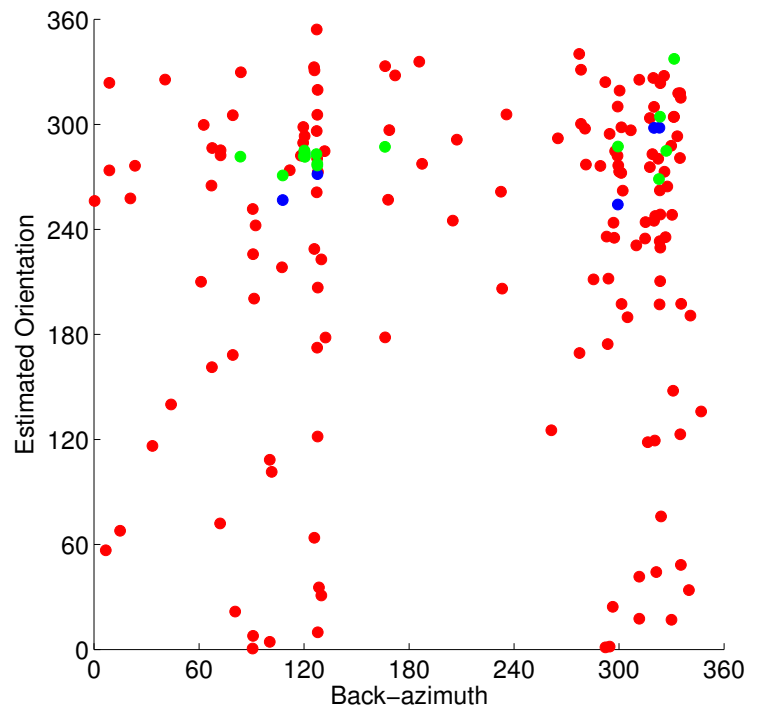

(d) Back azimuth versus orientation

Figure 4.1: Summary of NS12 orientation results. Green signifies estimates which have been manually graded 'good', blue indicates estimates which have been manually graded 'questionable', and red indicates estimates which have an automatic or manual 'bad' grade. Only "good" grade estimates are used in calculating the final orientation. 


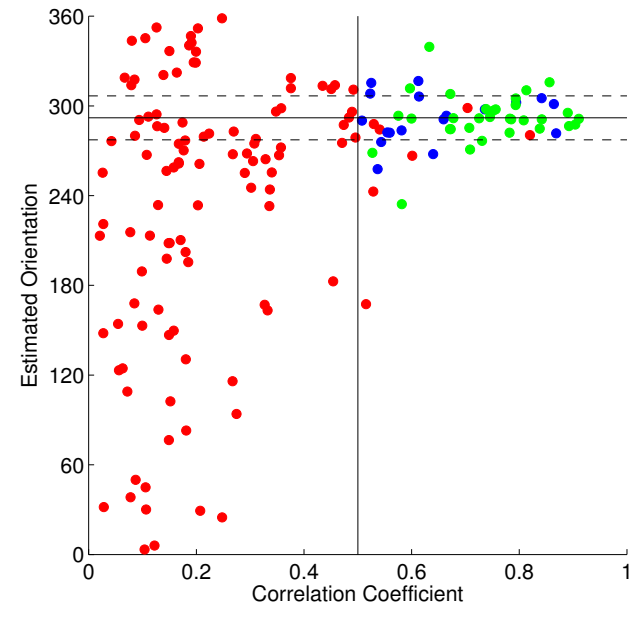

(a) Correlation versus orientation

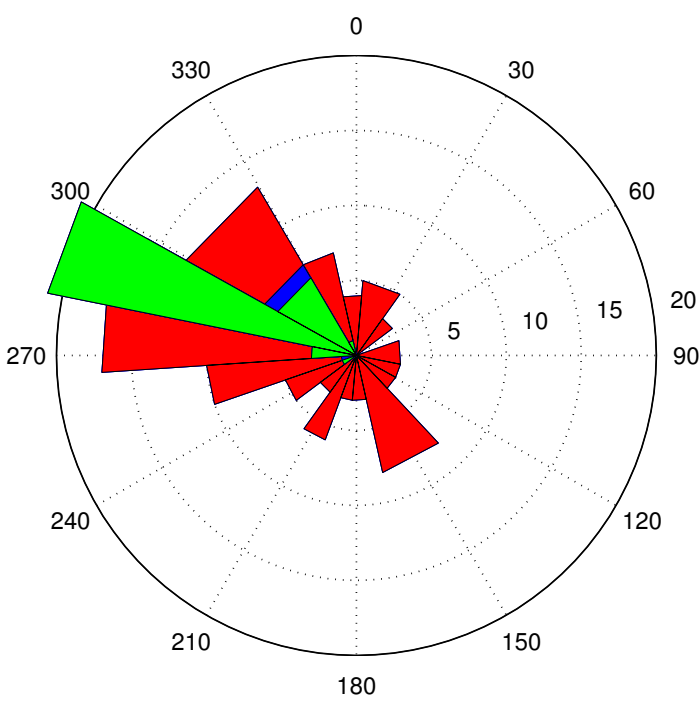

(c) Rose diagram of orientations

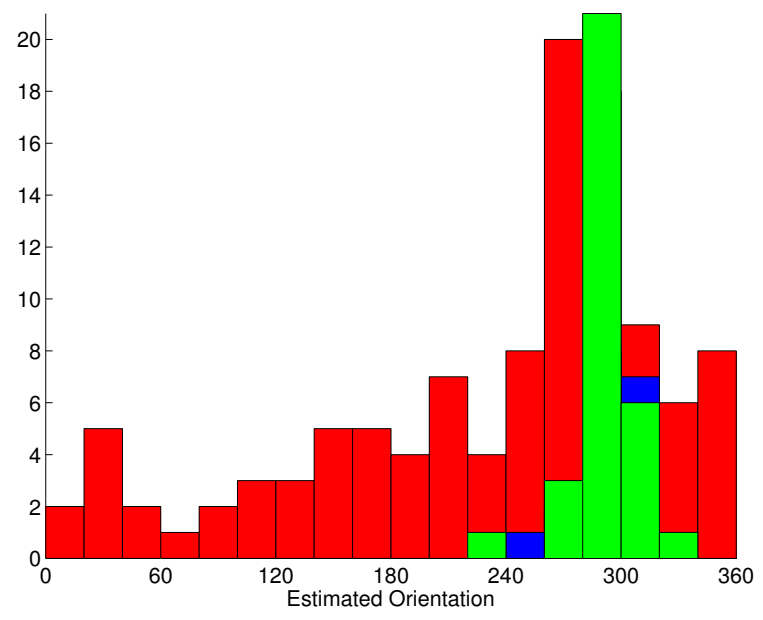

(b) Histogram of orientations

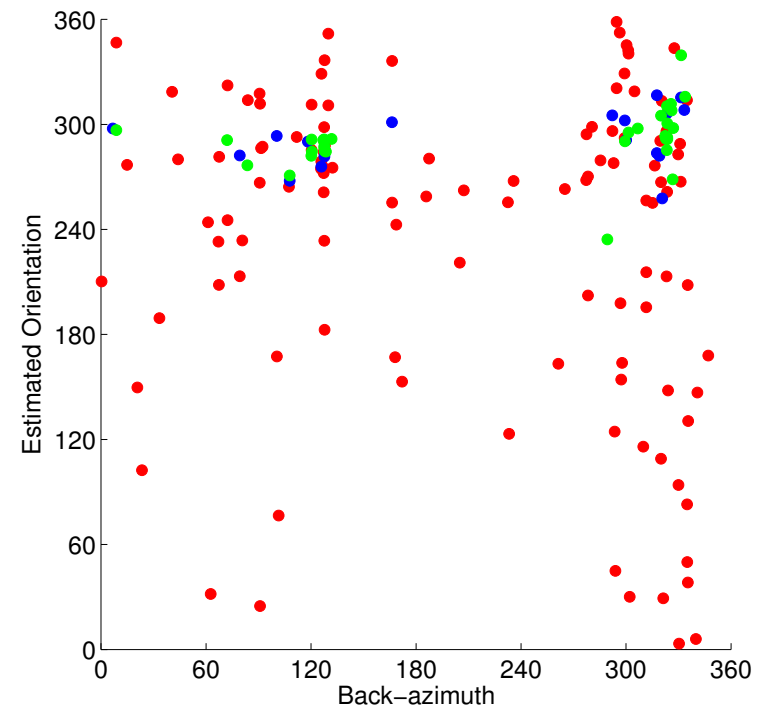

(d) Back azimuth versus orientation

Figure 4.2: Summary of NS13 orientation results. Green signifies estimates which have been manually graded 'good', blue indicates estimates which have been manually graded 'questionable', and red indicates estimates which have an automatic or manual 'bad' grade. Only "good" grade estimates are used in calculating the final orientation. 


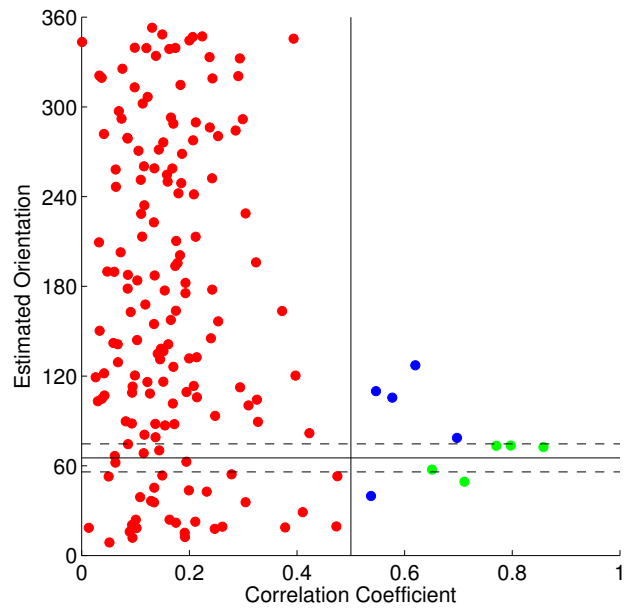

(a) Correlation versus orientation

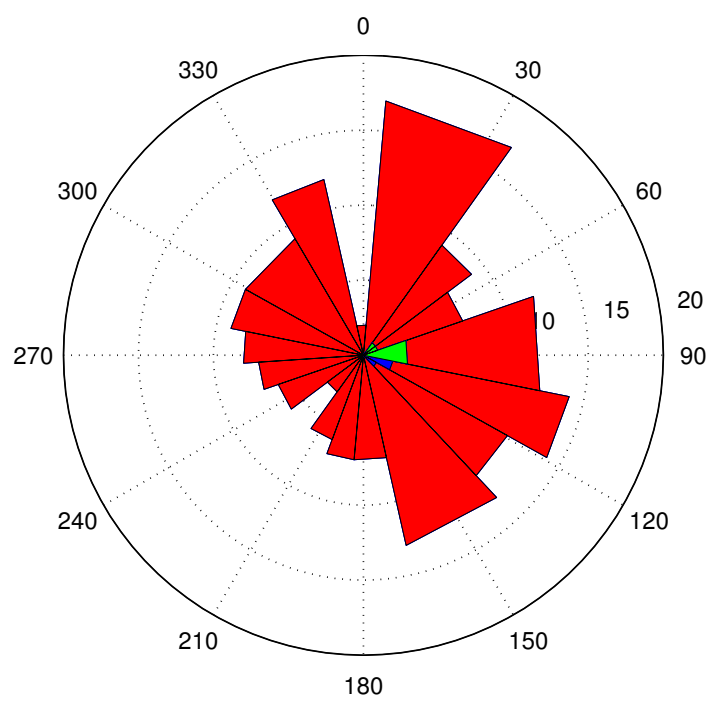

(c) Rose diagram of orientations

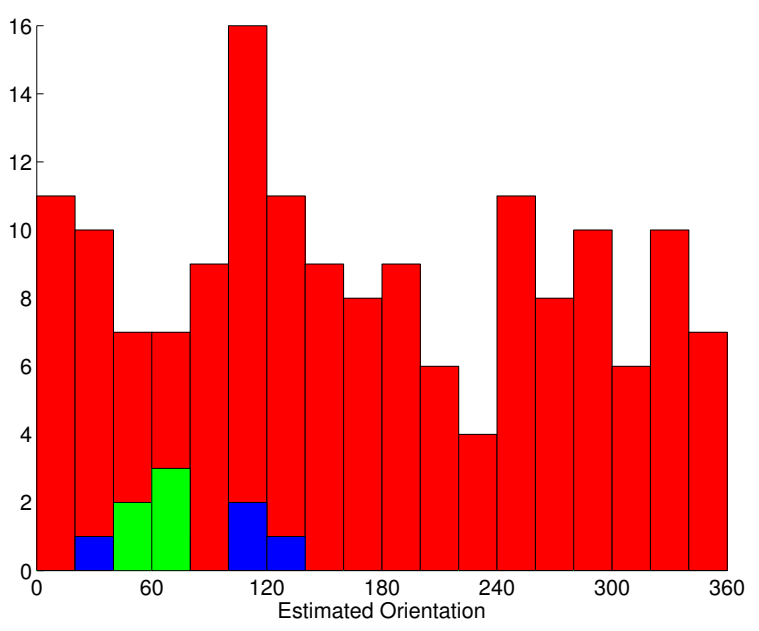

(b) Histogram of orientations

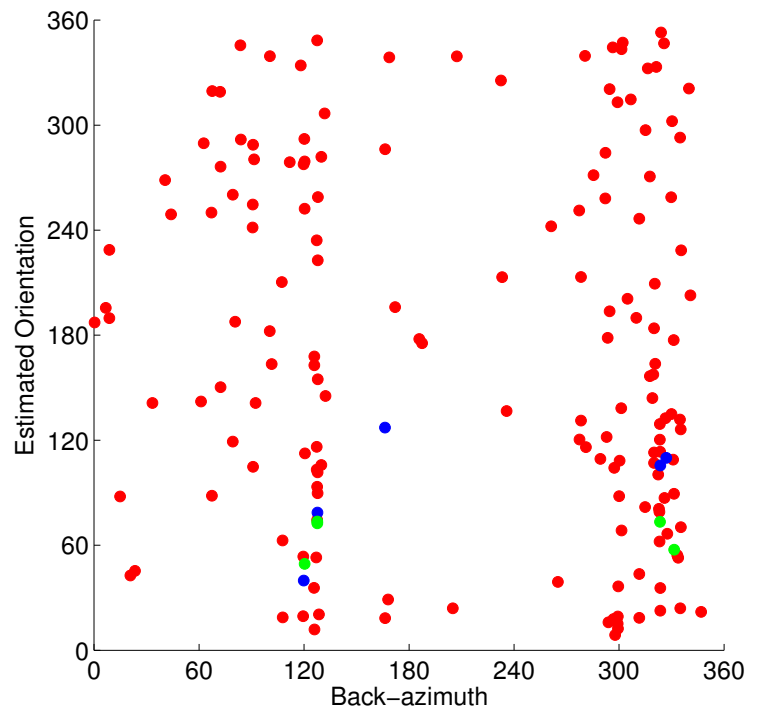

(d) Back azimuth versus orientation

Figure 4.3: Summary of NS14 orientation results. Green signifies estimates which have been manually graded 'good', blue indicates estimates which have been manually graded 'questionable', and red indicates estimates which have an automatic or manual 'bad' grade. Only "good" grade estimates are used in calculating the final orientation. 


\subsection{Shear wave picking (spickerC)}

The $v_{P} / v_{S}$ band chosen for shear wave picking appears to capture the full range of S-wave arrival times as we see a full normal distribution of $v_{P} / v_{S}$ (see Section 3.10).

Approximately half of our events are detected using the matched filter technique (Section 1.5.3). However, as might be expected, matched filter events have a much lower picking rate of $10 \%$ versus the $17 \%$ for GNS catalogue events. Events detected solely with the matched filter technique have avoided detection by conventional methods and thus are much more likely to be of lower magnitude and to have lower signal to noise ratio (making S-wave picking harder).

\subsubsection{Errors in S-wave picks}

The maximum error setting on class0 picks made by spicker $C$ is 0.3 seconds. This value is close to the maximum allowable time delay of 0.4 seconds (in MFAST processing) and double the 0.16 seconds maximum delay time we allow for high grade shear wave splitting measurements.

We allow such a high error threshold for our S-wave picking because MFAST makes its shear wave splitting measurements on windows around the S-wave pick and uses various comparisons to select the best window (Section 3.5.2). Because the S-wave pick is only used indirectly in setting up the windows, a shear wave splitting measurement is not highly sensitive to the pick time. In addition, most S-wave picks have errors well below 0.3 seconds (Figure 4.4). The error threshold and these quoted errors are the combined error for all three picking methods used by spickerC. 


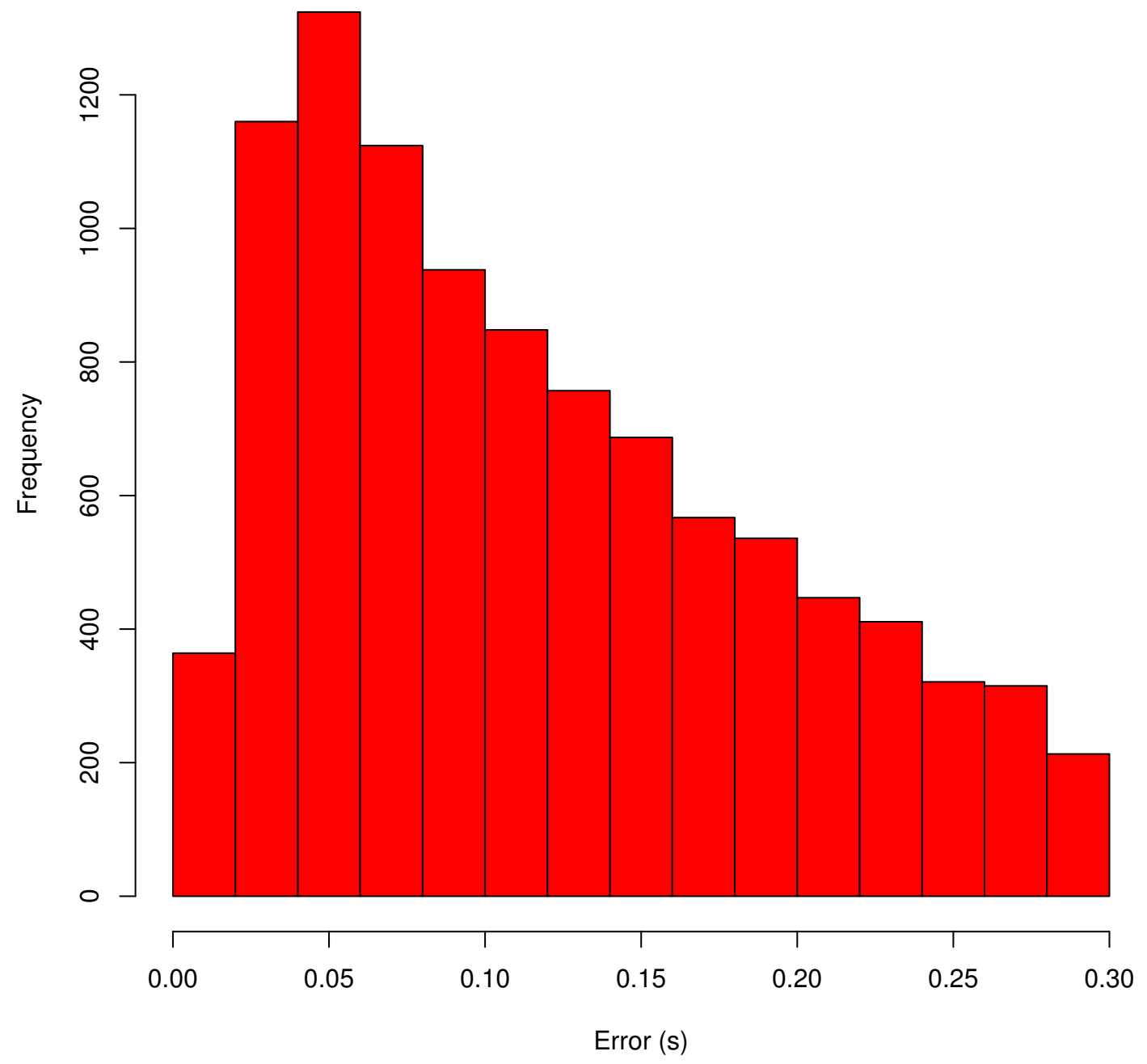

Figure 4.4: Histogram of S-wave picking uncertainty for class0 picks (which are later used in shear wave splitting processing). 


\subsection{Shear wave splitting (stations)}

Per station means of fast polarisation $(\phi)$ and delay time $(\delta t)$ are summarised in Table 4.2 and Table 4.3. We divide stations into two groups; those which pass the Rayleigh test (Section 3.2.3) with a p-value less than 0.05, and those which fail the Rayleigh test $(\mathrm{p}$-value $\geq 0.05)$. Standard errors for $\phi_{\text {mean }}$ are calculated with a bootstrap method (Section 3.2.7). Figure 4.5 shows the rose diagrams plotted above their station locations.

Most stations outside of Rotokawa have mean fast polarisations within $010^{\circ}$ of the regional direction of $S_{H_{\max }}$ of NE-SW (Townend et al. 2012). Within Rotokawa, stations which pass the Rayliegh test, appear to have mean fast polarisations around a N-S orientation.

\subsubsection{Assumptions}

Averages of shear wave splitting parameters or percent anisotropy averaged across each station assume that each station is sampling from a single homogeneous anisotropic body (with hexagonal symmetry) which is invariant through time. This is an assumption we attempt to move away from by clustering earthquakes by their station-event paths (Section 3.7). It also assumes that angles of propagation are sub-vertical to the crack plane (i.e. parameters don't change with propagation angle, Section 2.2). We address this by fitting crack planes (Section 3.8).

\subsubsection{Percent anisotropy}

If we assume that time delay is evenly accumulated along the entire raypath, we can estimate the percent anisotropy, $k$, (also known as coefficient of anisotropy Babuska and Cara (1991)) for the minimum and maximum shear wave velocities $\left(\beta_{\text {min }}\right.$ and 
Table 4.2: Station means for shear wave splitting measurements for stations with dominant mean orientations (i.e. pass the Rayleigh test with a p-value $\leq 0.05$ ). S.E. and S.D. are standard deviation and standard error respectively. Borehole stations are marked with $*$.

\begin{tabular}{|c|c|c|c|c|c|c|c|c|}
\hline Station & $\phi\left(^{\circ}\right)$ & S.D. $\left(^{\circ}\right)$ & S.E. $\left(^{\circ}\right)$ & p-value & $\delta t(\mathrm{~s})$ & S.D. (s) & S.E. (s) & No. \\
\hline \hline ARAZ & 30 & 36 & 9.3 & 0.0073 & 0.11 & 0.041 & 0.0086 & 23 \\
\hline NS01 & 51 & 45 & 5.3 & $3.4 \mathrm{e}-06$ & 0.098 & 0.039 & 0.0032 & 147 \\
\hline NS03 & 38 & 40 & 4.8 & $5.2 \mathrm{e}-09$ & 0.092 & 0.037 & 0.0032 & 134 \\
\hline NS11 & 83 & 34 & 6.5 & 0.00044 & 0.092 & 0.035 & 0.0063 & 31 \\
\hline NS13* & 55 & 37 & 4.2 & $7.1 \mathrm{e}-09$ & 0.076 & 0.038 & 0.0039 & 97 \\
\hline NS14 & 13 & 52 & 11 & 0.023 & 0.086 & 0.036 & 0.0035 & 108 \\
\hline NS16 & 41 & 29 & 2.5 & $2.9 \mathrm{e}-22$ & 0.072 & 0.053 & 0.0044 & 143 \\
\hline RT01 & 14 & 42 & 2.6 & $7.1 \mathrm{e}-24$ & 0.087 & 0.043 & 0.002 & 453 \\
\hline RT05 & 50 & 54 & 5 & $2.2 \mathrm{e}-07$ & 0.065 & 0.036 & 0.0015 & 570 \\
\hline RT12 & -18 & 30 & 1.8 & $6.6 \mathrm{e}-35$ & 0.11 & 0.038 & 0.0025 & 228 \\
\hline RT17 & 38 & 46 & 5.2 & $7.3 \mathrm{e}-08$ & 0.094 & 0.034 & 0.0022 & 226 \\
\hline RT21 & -40 & 25 & 5.8 & $4.5 \mathrm{e}-05$ & 0.11 & 0.039 & 0.009 & 19 \\
\hline RT22 & -31 & 34 & 9 & 0.012 & 0.085 & 0.035 & 0.0084 & 18 \\
\hline WPRZ & 41 & 38 & 2.6 & $1.7 \mathrm{e}-19$ & 0.086 & 0.044 & 0.0028 & 245 \\
\hline
\end{tabular}


Table 4.3: Station means for shear wave splitting measurements for stations without dominant mean orientations (i.e. fail the Rayleigh test with a p-value > 0.05). S.E. and S.D. are standard deviation and standard error respectively. Borehole stations are marked with $*$.

\begin{tabular}{|c|c|c|c|c|c|c|c|c|}
\hline Station & $\phi\left(^{\circ}\right)$ & S.D. $\left(^{\circ}\right)$ & S.E. $\left(^{\circ}\right)$ & p-value & $\delta t(\mathrm{~s})$ & S.D. $(\mathrm{s})$ & S.E. (s) & No. \\
\hline \hline NS04 & 83 & 1.5 & 1.1 & 0.14 & 0.11 & 0.0018 & 0.0013 & 2 \\
\hline NS07 & -62 & 52 & 23 & 0.33 & 0.098 & 0.038 & 0.0071 & 29 \\
\hline NS12 & -56 & 77 & 53 & 0.96 & 0.083 & 0.035 & 0.0046 & 58 \\
\hline NS15 & 37 & 74 & 52 & 0.95 & 0.08 & 0.04 & 0.0063 & 40 \\
\hline NS18 & 8.3 & 47 & 17 & 0.18 & 0.069 & 0.029 & 0.0058 & 25 \\
\hline RT14 & 54 & 54 & 20 & 0.22 & 0.073 & 0.037 & 0.005 & 54 \\
\hline RT18 & 44 & 71 & 29 & 0.54 & 0.072 & 0.037 & 0.0023 & 263 \\
\hline RT19 & 67 & 58 & 38 & 0.8 & 0.086 & 0.052 & 0.013 & 15 \\
\hline RT23 & 0.12 & 43 & 17 & 0.14 & 0.11 & 0.025 & 0.0058 & 18 \\
\hline
\end{tabular}




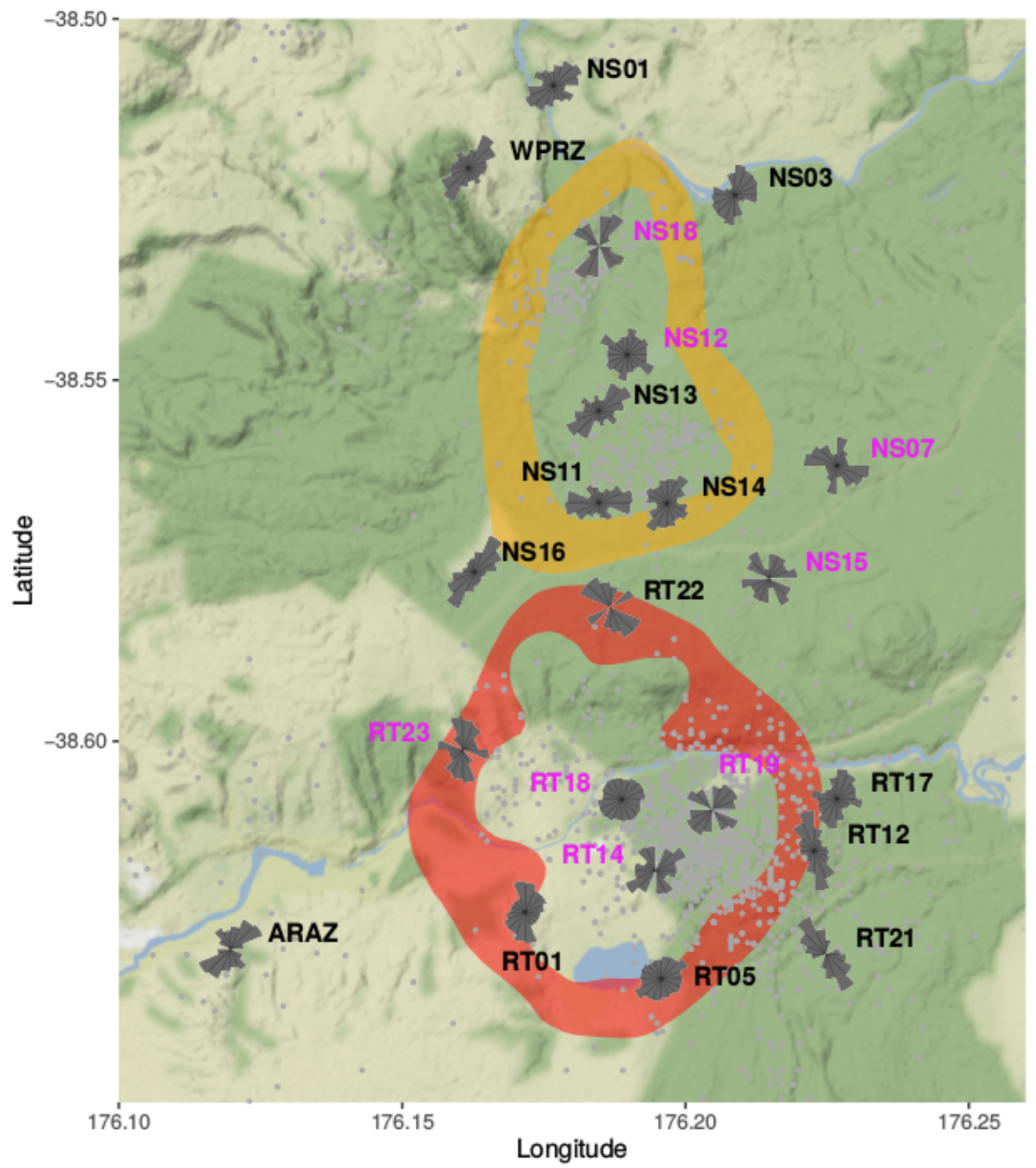

Figure 4.5: Rose diagrams of per station fast polarisations ( $\phi) . \mathrm{NSO}_{4}$ is removed due to only having two measurements (due seismometer malfunction). A purple label indicates that the has failed the Rayleigh test ( $p$-value $>0.05)$. Stations may fail the Rayleigh test but still carry visual information (i.e. have multimodal distributions). 
$\left.\beta_{\max }\right)$ as well as average shear wave velocity $(\bar{\beta})$.

$$
k=\frac{\beta_{\max }-\beta_{\min }}{\bar{\beta}} * 100
$$

We also quote differential shear wave anisotropy, $S W A$, (Crampin 1989)

$$
S W A=\frac{\beta_{\max }-\beta_{\min }}{\beta_{\max }} * 100
$$

Table A.5 summarises both $k$ and $S W A$ for each station. This is likely an underestimate of the true percentage of anisotropy as it does not take into account time delays varying with propagation angle and that the time delay is measured across the full station-earthquake path distance. Mean values are:

$$
\begin{gathered}
\bar{k}=3.47 \% \\
S \bar{W} A=3.39 \%
\end{gathered}
$$

Neither $k$ nor $S W A$ depend on the distance travelled by the wave (through a homogeneous anisotropic body). If $k$ or $S W A$ are decreasing with distance travelled then time delay may not be being accumulated along the entire path (i.e. the anisotropic body only covers a portion of the total path). Thus, we expect zero correlation between distance travelled and percent anisotropy. We find that the lowest average (absolute) correlation coefficient occurs when $\beta_{\min }$ is estimated for path lengths of $4.9 \mathrm{~km}$ (i.e. distance travelled is capped at $4.9 \mathrm{~km}$ ). $\beta_{\max }$ is still estimated across the full path. These estimates of percent anisotropy are also quoted in Table A.5. Figure 4.6 shows station-event distance versus percent anisotropy for percentages calculated across the entire raypath and for raypaths capped at $4.9 \mathrm{~km}$. Mean values for the capped raypaths are:

$$
\begin{gathered}
\bar{k}=4.11 \% \\
S \bar{W} A=4.0 \%
\end{gathered}
$$




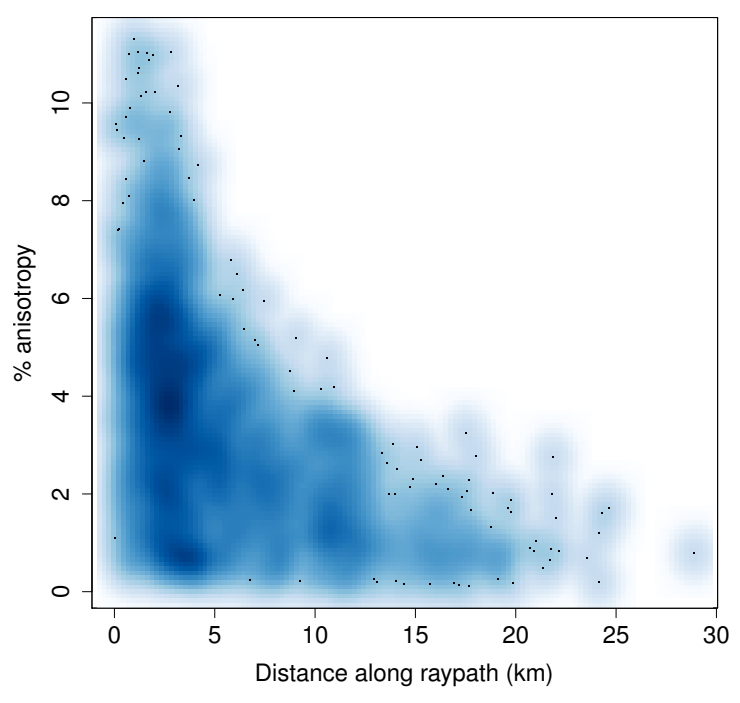

(a) Full raypath

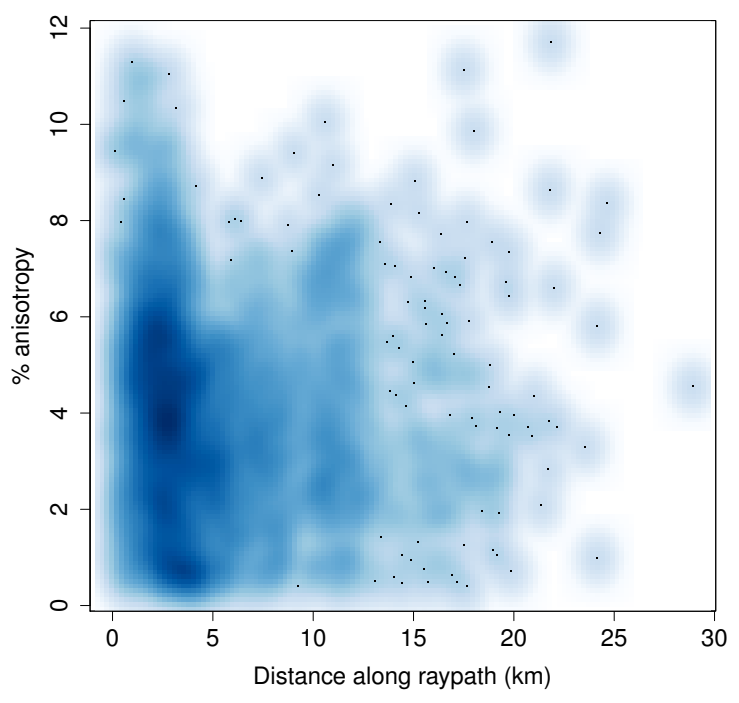

(b) Raypath capped at $4.9 \mathrm{~km}$

Figure 4.6: Percent anisotropy versus station-event path distance across all stations. b) shows the estimated percent anisotropy if anisotropy is restricted to $4.9 \mathrm{~km}$ of the path distance.

Both these estimates of percent anisotropy fall into the upper bound expected for cracks in the upper 10-15 km of the crust for intact rock (Savage 1999; Crampin and Peacock 2008).

This value of roughly $4 \%$ agrees with percent anisotropy measured at other geothermal fields (e.g. Evans et al. 1995; Lou and Rial 1997).

\subsubsection{Matched filter detections versus templates}

$53 \%$ of our measurements are made from earthquakes detected by the matched filter method (Section 1.5.3) which, with a slightly lower picking and splitting rate, corresponds to $44 \%$ of our high grade shear wave splitting measurements.

Each matched filter earthquake has a corresponding template earthquake. The 
shear wave splitting parameters for each template correlate somewhat strongly with those for their corresponding detections (Section 1.5.3). Correlation coefficients between templates and their corresponding detections are, for $\delta t$ and $\phi$ respectively, 0.53 and 0.55 (using the correlation described in Section 3.2.5). This relationship is illustrated in Figure 4.7.

A template with $100 \%$ correlation with its detection must have the same shear wave splitting parameters. As matched filter detections are based on correlations (and thus have highly similar waveforms), a template and its detection are much more likely to have the same shear wave splitting parameters than two random events (both measured on the same station). In addition, detections and their templates tend to occur together (both spatially and temporally) and thus are likely to be sampling the same anisotropy with a similar raypath.

\subsubsection{Rayleigh test failures}

Several station in Rotokawa, located directly on top of the majority of the seismicity, fail the Rayleigh test (RT14, RT18 and RT19). Being located almost directly above the majority of the seismicity across both fields means that the station-event paths from each earthquake differ a lot when compared to stations off set from the seismicity.

This could result in these stations failing the Rayleigh test (Section 3.2.3) for two reasons. Firstly, measurements with steeper propagation angles with respect to the symmetry axis of the anisotropy may be at $90^{\circ}$ to the crack orientation (see Section 2.4.1) which may be a factor for RT14 and RT19 after visual inspection. Secondly, if anisotropy is changing rapidly within Rotokawa (which there is some evidence for, see Section 1.4.2) then earthquakes either side of a station could be sampling differing crack planes. Whereas, when a station is offset from the seismicity, station-event paths will be similar, especially closer to the station where the majority of measured 


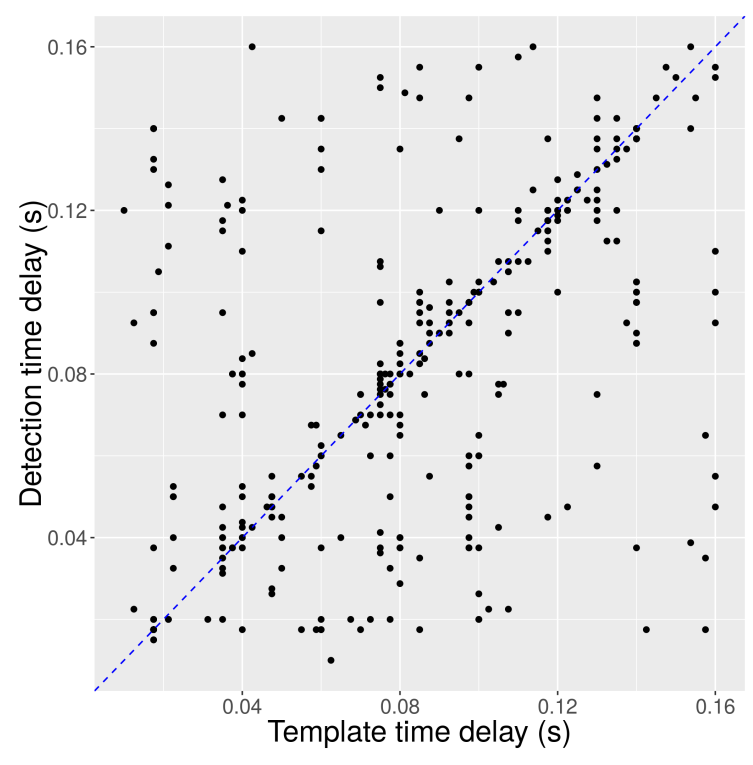

(a) Time delay

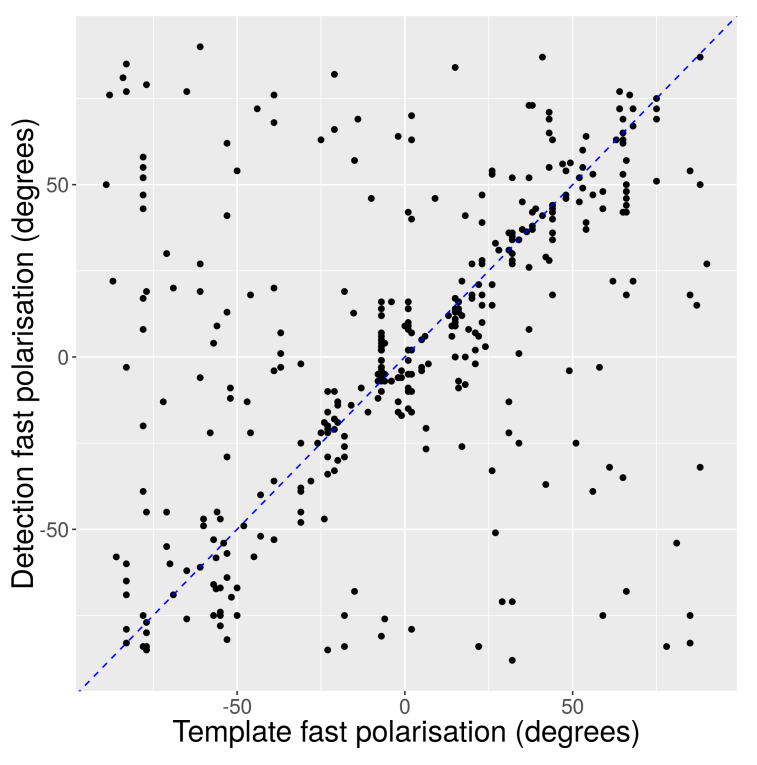

(b) Fast polarisation

Figure 4.7: Relationship between time delay and fast polarisation between a template event and their corresponding matched filter detections (measured on the same station). Correlation coefficients for time delay and fast polarisation are 0.53 and 0.55 respectively. 
anisotropy is thought to originate (Rümpker and Silver 2000; Nistala and McMechan 2005). Other stations (outside of Rotokawa) may also be failing the Rayleigh test for similar reasons.

We address both these mechanisms, by which stations may be failing the Rayleigh test, by clustering earthquakes by incoming angle and back azimuth (Section 3.7.1) (to account for local changes in anisotropy) and by fitting clusters to theoretical crack planes (Section 3.8) (to account for steep propagation angles).

Of these stations which fail the Rayleigh test, only RT18 has a large number of shear wave splitting measurements (Table 4.3). It is also possible that many of these stations are failing simply because their underlying population has low concentration around its mean and so they have not sampled enough measurements to return a statistically significant mean polarisation.

\subsubsection{Rotokawa in-situ cracks}

Massiot et al. (2015) identifies four families of cracks in the Rotokawa boreholes (RK18L2, RK32, and RK30L1). The largest family (and one secondary family in RK32) strikes NE-SW and is present in all three wells. A secondary family present in RL18L2 and RK30L1 strikes N-S along with a family in RK30L1 striking NW-SE. The strike of these families is similar to the polarisations we measure with shear wave splitting.

These two secondary groups of cracks striking N-S and NW-SE are particularly interesting as we observe shear wave splitting fast polarisations within Rotokawa which fall broadly into these two categories. If there is a relationship between frequency of earthquake data and the scale of cracks which shear wave splitting is sensitive to (Section 2.3.4), it may be possible to gain evidence for this frequency relationship since our measurements (in Rotokawa) appear to be sensitve to the N-S family. However, apart from differences in fracture spacing distribution which Mas- 
siot et al. (2015) suggests is because the fractures are tectonic, there is no noted scale difference between these observed crack families.

McNamara et al. (2015) find some positive correlation between permeable zones, within the boreholes, and crack density and crack aperture. Thus, these permeable zones may equate to areas with higher anisotropy.

As an avenue for future work we could attempt to construct an elasticity tensor for a rock which contains these known families of cracks and thus be able to fit our shear wave splitting measurements to a more accurate representation of the known cracks.

\subsection{Crack fitting}

We apply our crack fitting method (Section 3.8) to path clustered groups of shear wave splitting measurements (Section 3.7). By clustering our measurements by station-event path, we relax the assumption that measurements are sampling from the same anisotropic body. By taking these path clustered measurements and fitting them to theoretical crack planes we no longer have to assume that propagation angles are sub-vertical and that the cracks are vertical. Using the best fitting crack planes, we can restrict our dataset to include just those shear wave splitting measurements that do fulfil the assumption of sub-vertical propagation angles (Section 3.9.1). Each "group" represents shear wave splitting measurements from one or more stations that a have similar fit to their corresponding crack plane (Section 3.8.2).

The final fitted crack planes with greater than 50 shear wave splitting measurements are listed in Table 4.4 in order of uniqueness of their fit. $90^{\circ}$ dip is defined as vertical (i.e. cracks with $90^{\circ}$ dip are vertical cracks). Due to the high scatter in delay times, crack dip is less well constrained than crack strike.

We apply Watson's non-parametric test (Section 3.2.4) to compare the restricted 


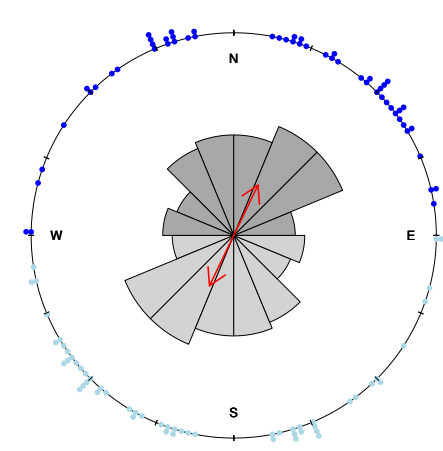

(a) Group 30.026 mean.

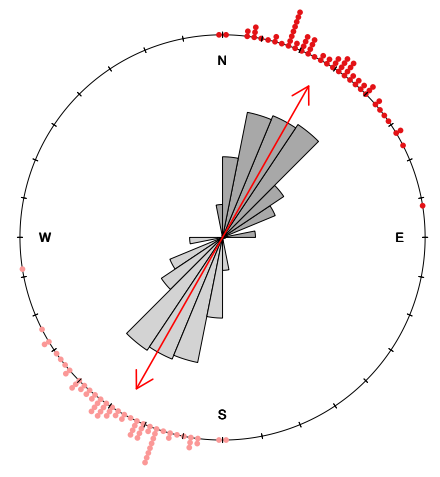

(b) RK32. $030^{\circ}$ mean.

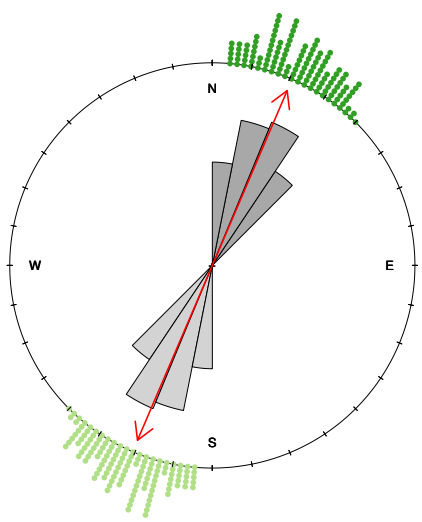

(c) RK30L1. 0230 mean.

Figure 4.8: In-situ estimates of $S_{H_{\max }}$ compared to Group 30 whose mean shows no significant evidence of differing from either RK32 or RK30L1 (p-value > 0.05).

measurements (Section 3.9.1) of each fitted crack plane to the in-situ stress measurements of McNamara et al. (2015) (described in Section 1.4.2). The result of this comparison is quoted in Table 4.4. Figure 4.8 illustrates the comparison for Group 30, whose mean shows no significant diffetrence from the of RK32 or RK30L1 $S_{H_{\max }}$ estimates.

Figure 4.10 shows the best fitting pole figure (with shear wave splitting measurements overlaid), misfit grid, and raypaths for the group with the highest uniqueness (Group 11). All the fitted crack planes in Table 4.4 are plotted in Appendix B.

\subsubsection{Summary}

Here we summarise the best fitting crack planes for those that have the best fit. Each "group" represents shear wave splitting measurements from one or more stations that have similar fit to their corresponding crack plane (Section 3.8.2). Figure 4.9 shows some of the best fitting crack planes with respect to their rough spatial location. 
Table 4.4: Table of fitted crack planes. For columns labelled with well name, "Yes" indicates no significant difference in mean between restricted polarisations and $S_{H_{\max }}$ measured in that well ( $p$-value $>0.05)$. NA indicates that either there weren't enough restricted measurements to do the comparison or the restricted measurements do not have a dominant mean orientation (fail the Rayleigh test). Mean delay times of the (restricted) groups are also given $(\delta t)$.

\begin{tabular}{|c|c|c|c|c|c|c|c|c|}
\hline & Strike $\left(^{\circ}\right)$ & Dip $\left(^{\circ}\right)$ & $\begin{array}{c}\text { Dip } \\
\text { Azimuth }\left(^{\circ}\right)\end{array}$ & $\delta t$ & RK18L2 & RK32 & RK30L1 & $\#$ \\
\hline \hline Group11 & 45 & 70 & 135 & 0.11 & Yes & Yes & No & 66 \\
\hline Group25 & 20 & 60 & 290 & 0.093 & No & No & No & 381 \\
\hline Group22 & 35 & 62.5 & 305 & 0.098 & No & No & No & 111 \\
\hline Group30 & 25 & 77.5 & 115 & 0.054 & No & Yes & Yes & 55 \\
\hline Group7 & 35 & 82.5 & 305 & 0.092 & No & Yes & No & 112 \\
\hline Group8 & -45 & 60 & 45 & 0.084 & NA & NA & NA & 69 \\
\hline Group14 & 0 & 70 & 90 & 0.081 & No & No & Yes & 55 \\
\hline Group38 & 35 & 87.5 & 305 & 0.096 & Yes & Yes & No & 98 \\
\hline Group28 & 80 & 62.5 & 350 & 0.08 & No & No & No & 123 \\
\hline Group18 & -45 & 65 & 45 & 0.092 & NA & NA & NA & 83 \\
\hline Group43 & 60 & 57.5 & 150 & 0.092 & NA & NA & NA & 81 \\
\hline Group26 & 75 & 40 & 165 & 0.092 & NA & NA & NA & 60 \\
\hline Group53 & 30 & 27.5 & 120 & 0.092 & NA & NA & NA & 53 \\
\hline Group34 & -20 & 65 & 70 & 0.103 & No & No & No & 179 \\
\hline Group37 & -65 & 22.5 & 25 & 0.092 & NA & NA & NA & 92 \\
\hline Group40 & -60 & 15 & 210 & 0.092 & NA & NA & NA & 108 \\
\hline Group3 & -40 & 65 & 50 & 0.142 & NA & NA & NA & 223 \\
\hline Group29 & 10 & 57.5 & 280 & 0.091 & NA & NA & NA & 129 \\
\hline Group31 & -60 & 52.5 & 30 & 0.092 & NA & NA & NA & 70 \\
\hline
\end{tabular}




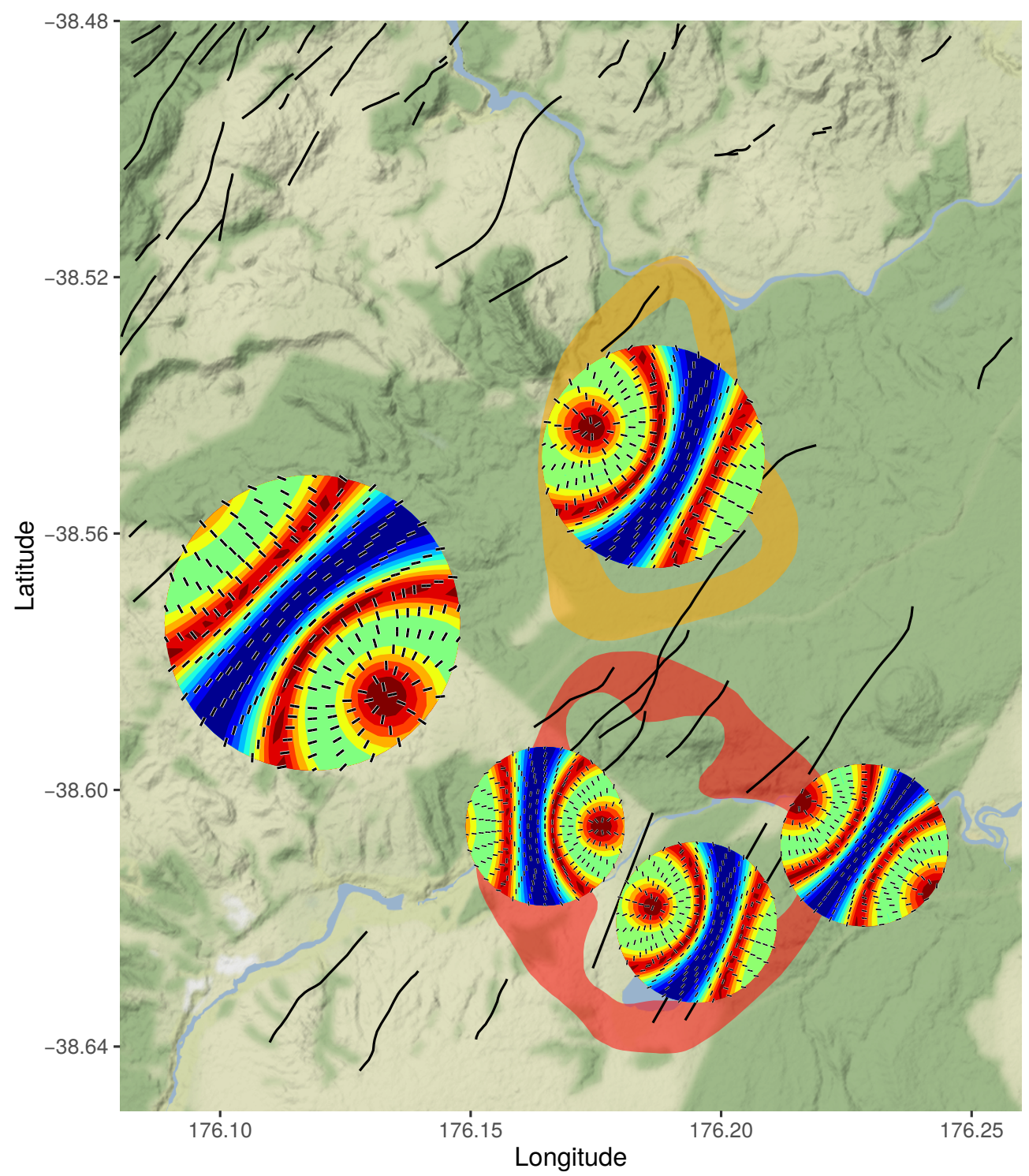

Figure 4.9: Lower hemisphere projections of theoretical anisotropy for the best fitting crack planes plotted over their rough spatial location. 


\section{Surrounding region}

Group 11 (Figure 4.10), the fit with the highest uniqueness, has station-event paths to the west of the study area outside of both fields. The orientation of this best fitting crack plane is $045^{\circ}$ which matches exactly with the NE-SW average orientation in the TVZ (Townend et al. 2012). For comparison, we also plot the commonly used spherical polar plot and equal area plot for Group 11's best fitting crack plane (Figure 4.11)

\section{Representative groups}

Group 22 (Figure 4.13), Group 7 (Figure 4.15), and Group 38 (Figure 4.16) all have high uniqueness and raypaths across Ngatamariki and central-eastern Rotokawa (for Group 38). These three groups have best fitting crack planes with strikes of $035^{\circ}$. $035^{\circ}$, while not strictly being NE-SW, is still within $5^{\circ}$ of what we would expect for the region.

Group 25 (Figure 4.12), for raypaths largely across central Rotokawa, has a best fitting crack strike of $020^{\circ}$. Group 30 (Figure 4.14), also has similar strike $\left(025^{\circ}\right)$ for raypaths in central-southern Rotokawa. Both these groups have low misfit $(<0.4)$ with high uniqueness. These groups represent what appears to be a rotation away from NE-SW toward N-S within Rotokawa. Group 25 is interesting as it is the group with the most unique fit which appears to fit measurements which have been flipped $90^{\circ}$ by their steep propagation angle. This change can be seen slightly more clearly on a spherical projection (Figure 4.17).

\section{Southern Ngatamariki}

Group 8 (Figure 4.18) contains two borehole stations (NS12 and NS14) with raypaths largely originating in Rotokawa. If these stations have not been oriented properly that could explain the $-045^{\circ}$ strike of the fitted crack plane. Or other factors relating 


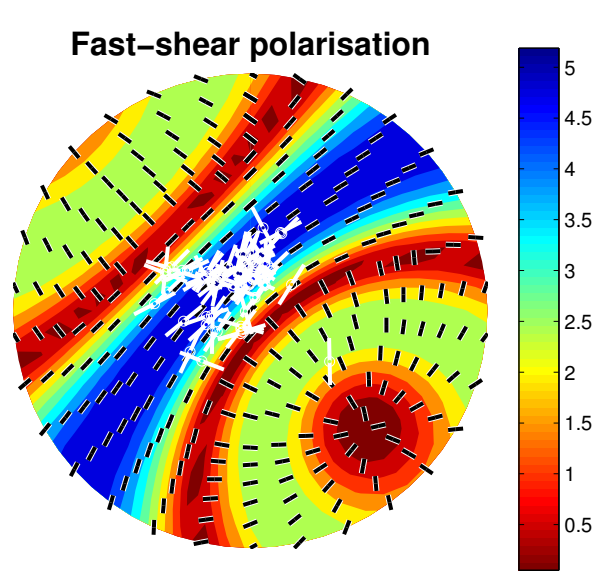

(a) Best fitting pole plot

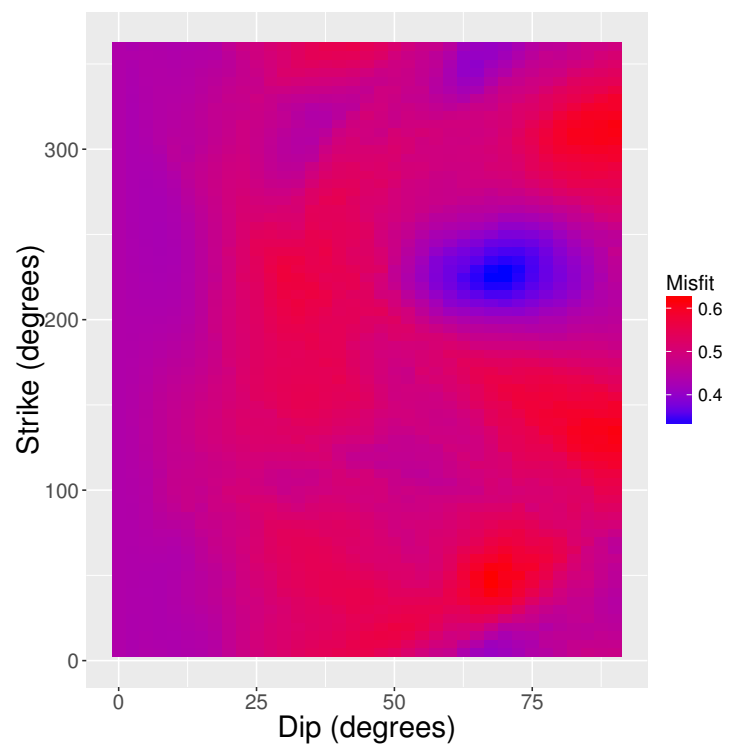

(b) Misfit grid

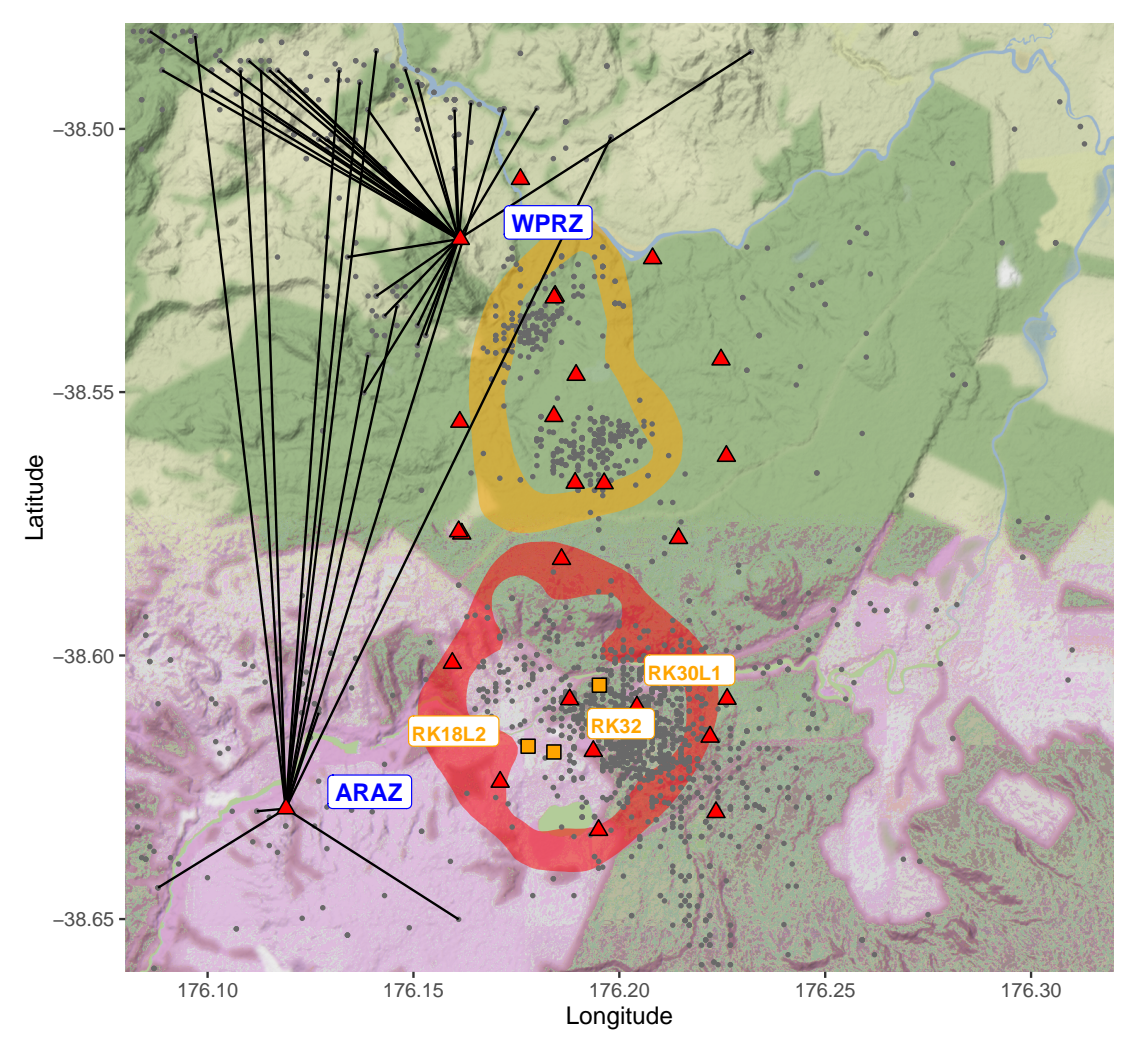

(c) Station-event paths for measurements in Group.

Figure 4.10: Summary of Group 11. Best fitting cracks striking 045․ 


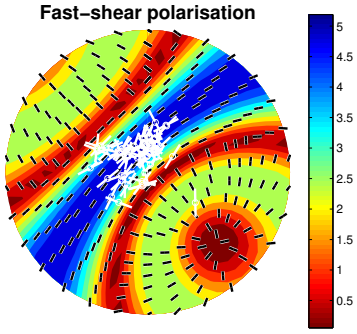

(a) Angle preserving

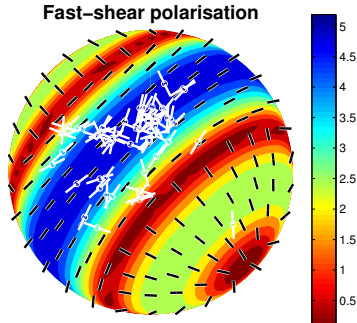

(b) Spherical

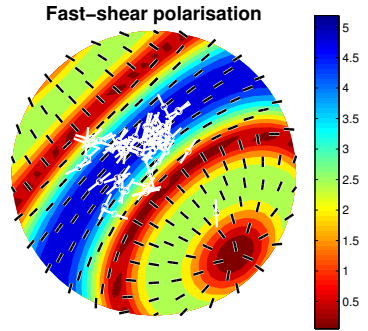

(c) Equal area

Figure 4.11: Angle preserving projection, spherical projection, and equal area projection for Group 11. As we focus on fitting orientations, we use angle preserving plots throughout this thesis.

to borehole stations may be affecting the polarisations (Section 5.2). Group 18 (Figure 4.19) contains raypaths which cross this same area from a surface station (NS16) and returns the same strike of $-045^{\circ}$ (and very similar dip) which may suggest that this $-045^{\circ}$ crack strike being seen in southern-central Ngatamariki is real. However, Group 18's polarisations are mostly $\sim 045^{\circ}\left(90^{\circ}\right.$ to the strike of the best fitting crack plane) which suggests the fit is likely incorrect.

\section{Rotokawa N-S}

Group 14 (Figure 4.20), with raypaths in northern Rotokawa, has a $0^{\circ}$ strike for its best fitting crack strike. This group represents the central orientation of groups, mostly located in Rotokawa, with strikes around N-S. Group 34 (Figure 4.21), with raypaths in central-eastern Rotokawa, despite having low uniqueness represents the other extreme for crack orientations within Rotokawa with a strike of $-020^{\circ}$ which appear to be grouped around a N-S orientation. 


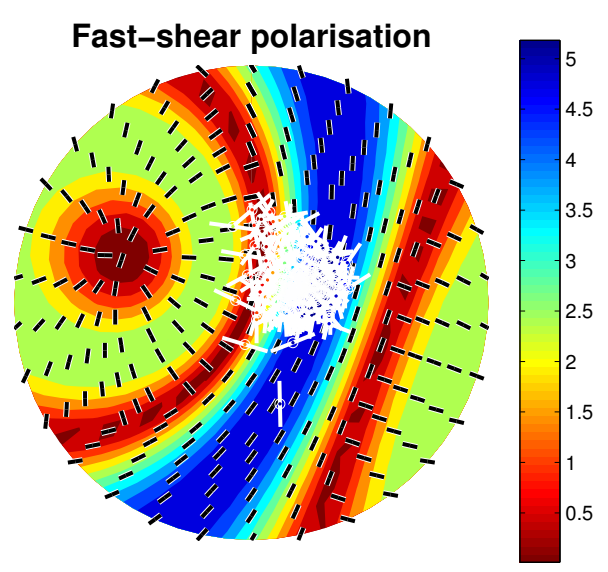

(a) Best fitting pole plot

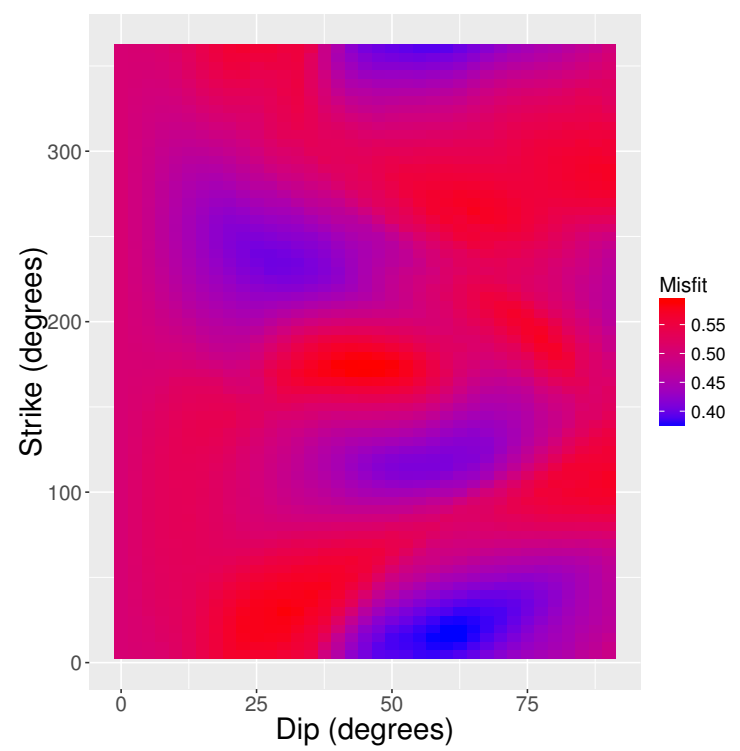

(b) Misfit grid

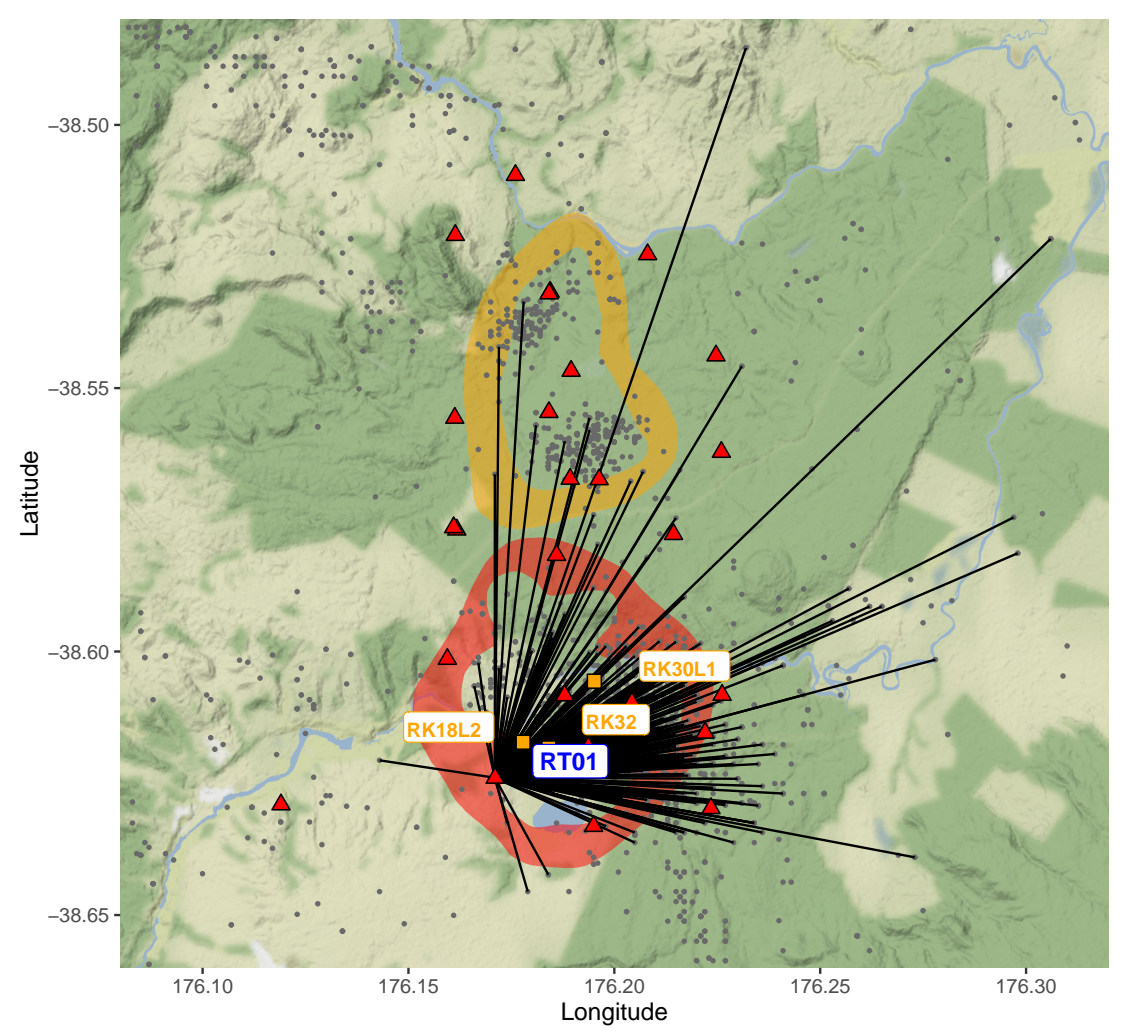

(c) Station-event paths for measurements in Group.

Figure 4.12: Summary of Group 25. Best fitting cracks striking 020 . 


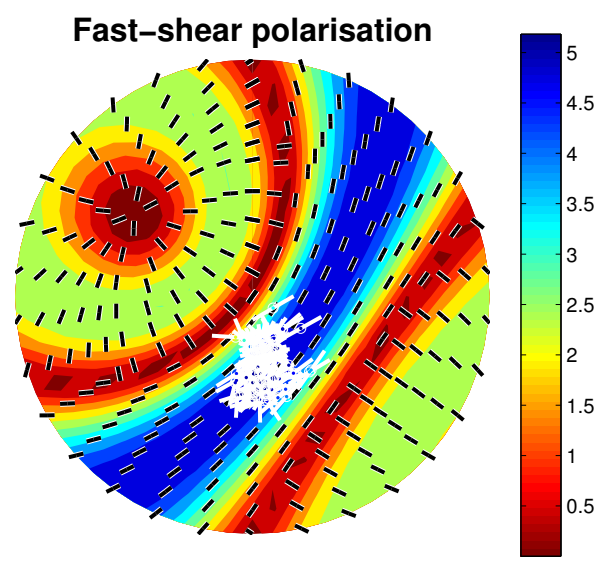

(a) Best fitting pole plot

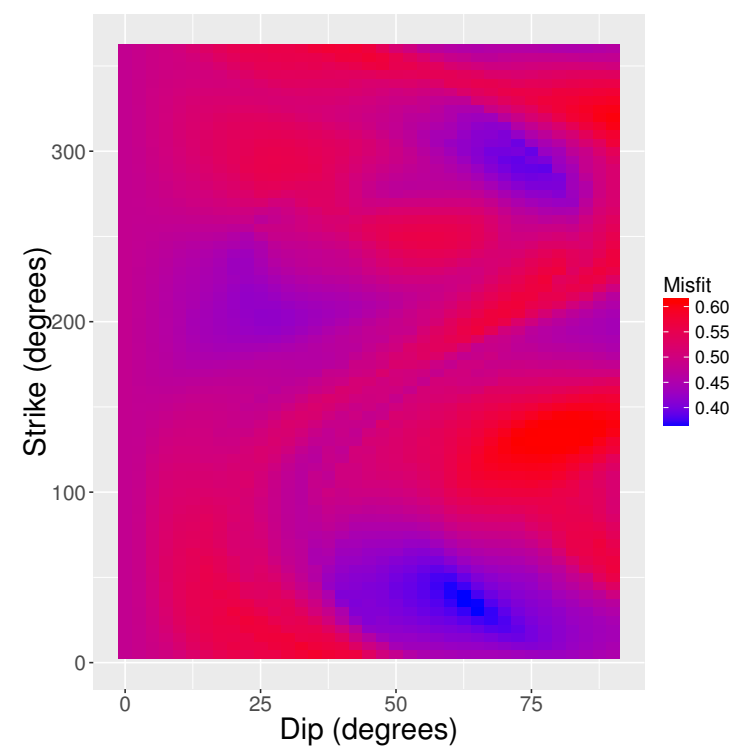

(b) Misfit grid

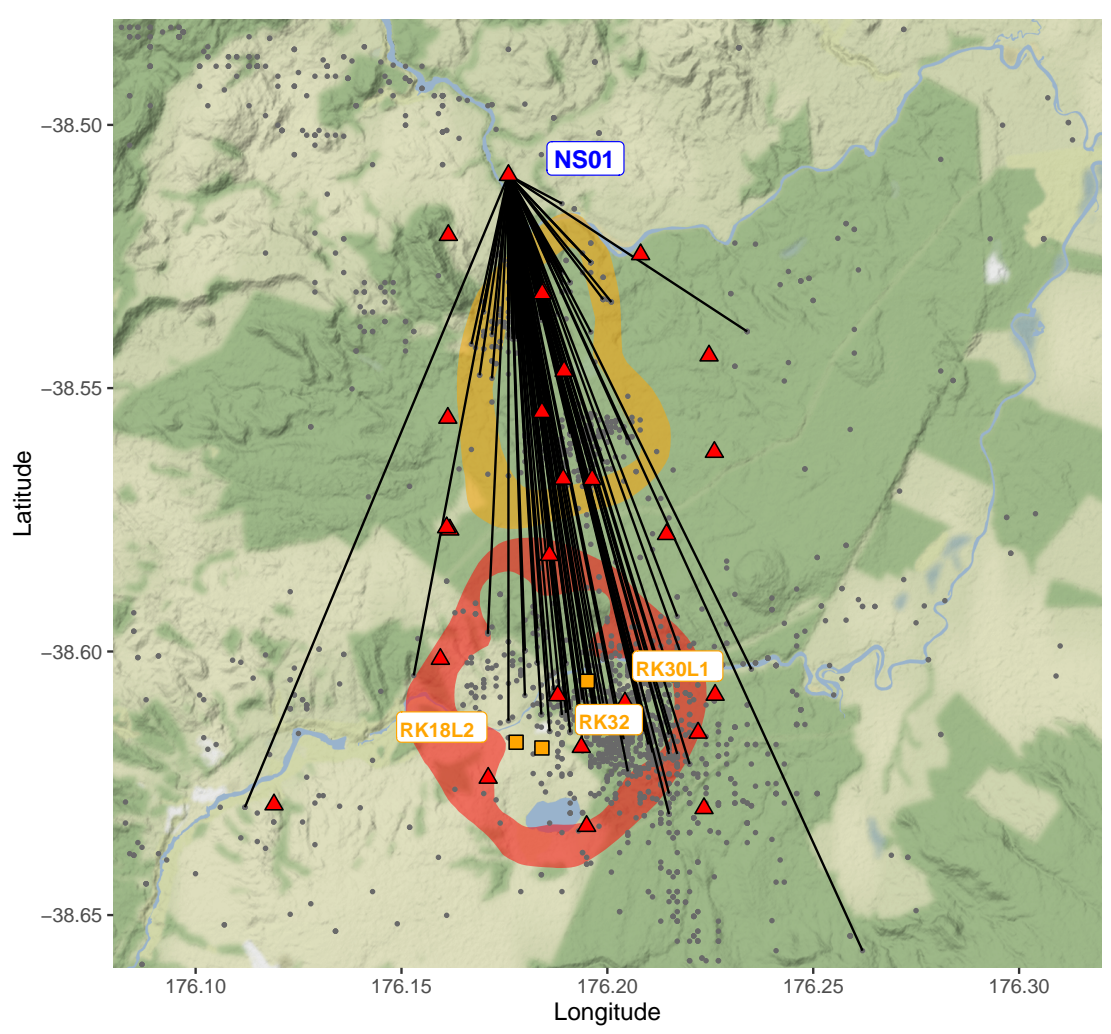

(c) Station-event paths for measurements in Group.

Figure 4.13: Summary of Group 22. Best fitting cracks striking 035. 


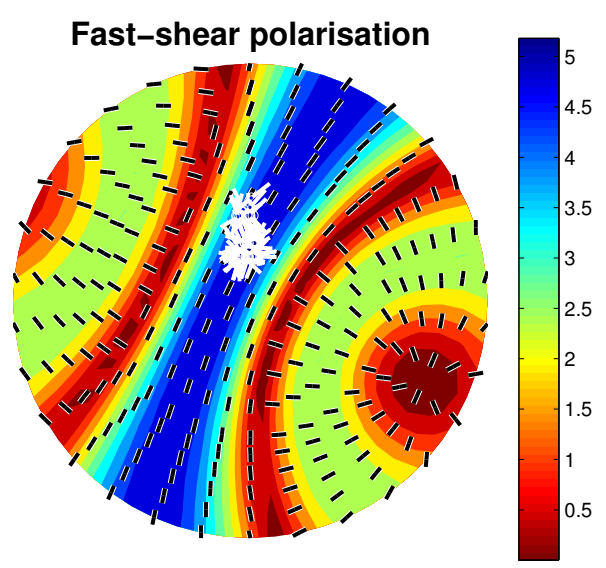

(a) Best fitting pole plot

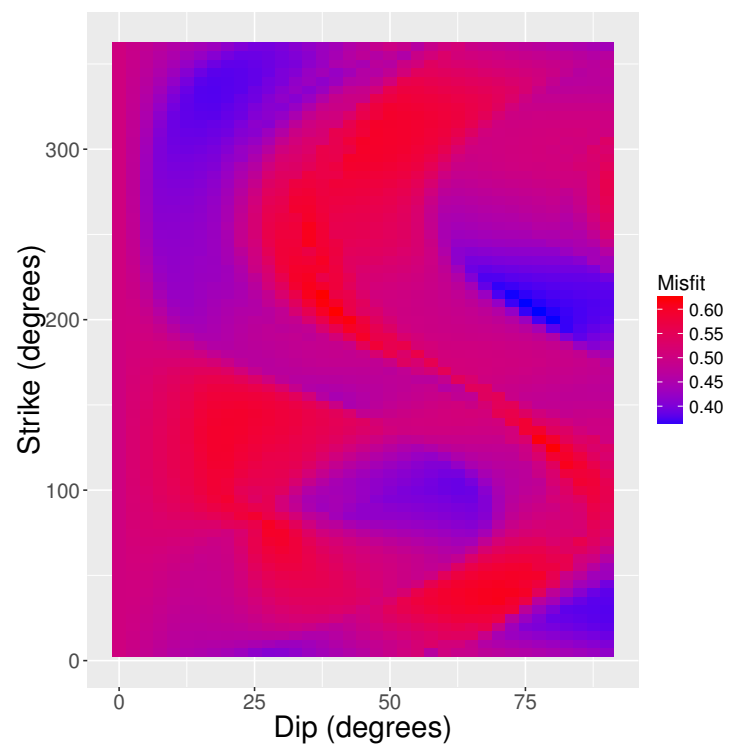

(b) Misfit grid

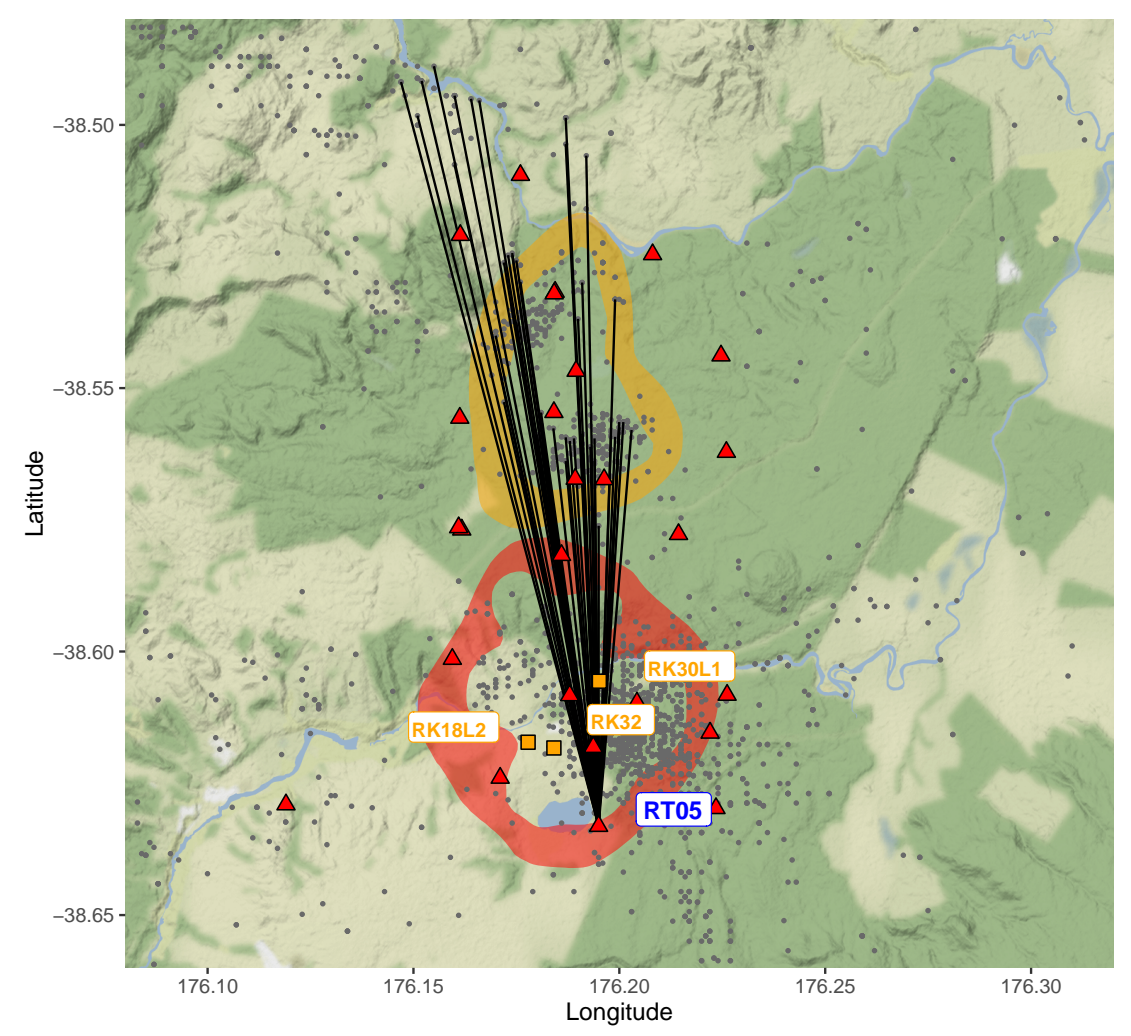

(c) Station-event paths for measurements in Group.

Figure 4.14: Summary of Group 30. Best fitting cracks striking 025․ 


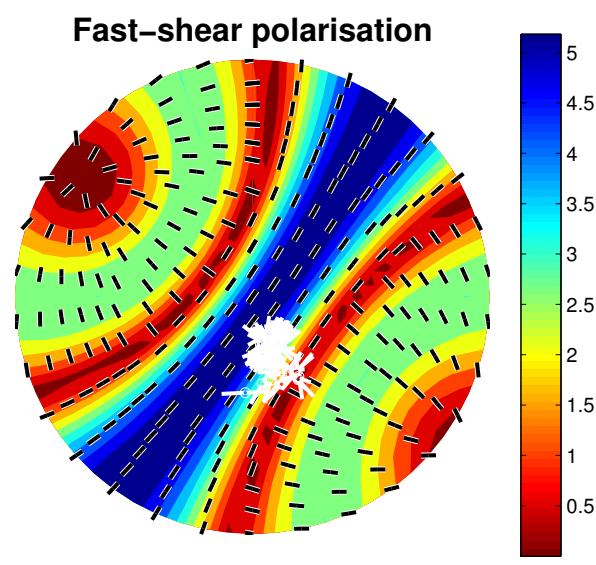

(a) Best fitting pole plot

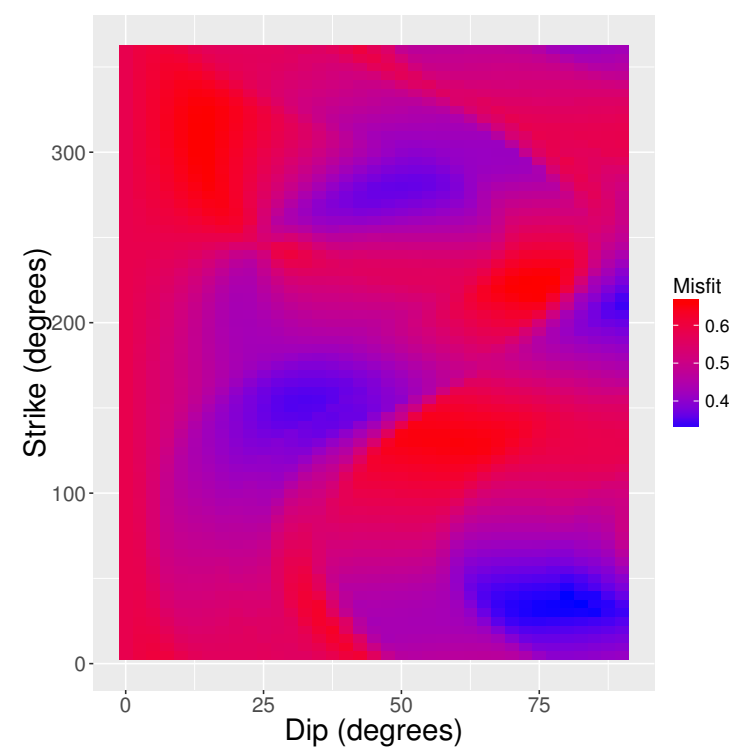

(b) Misfit grid

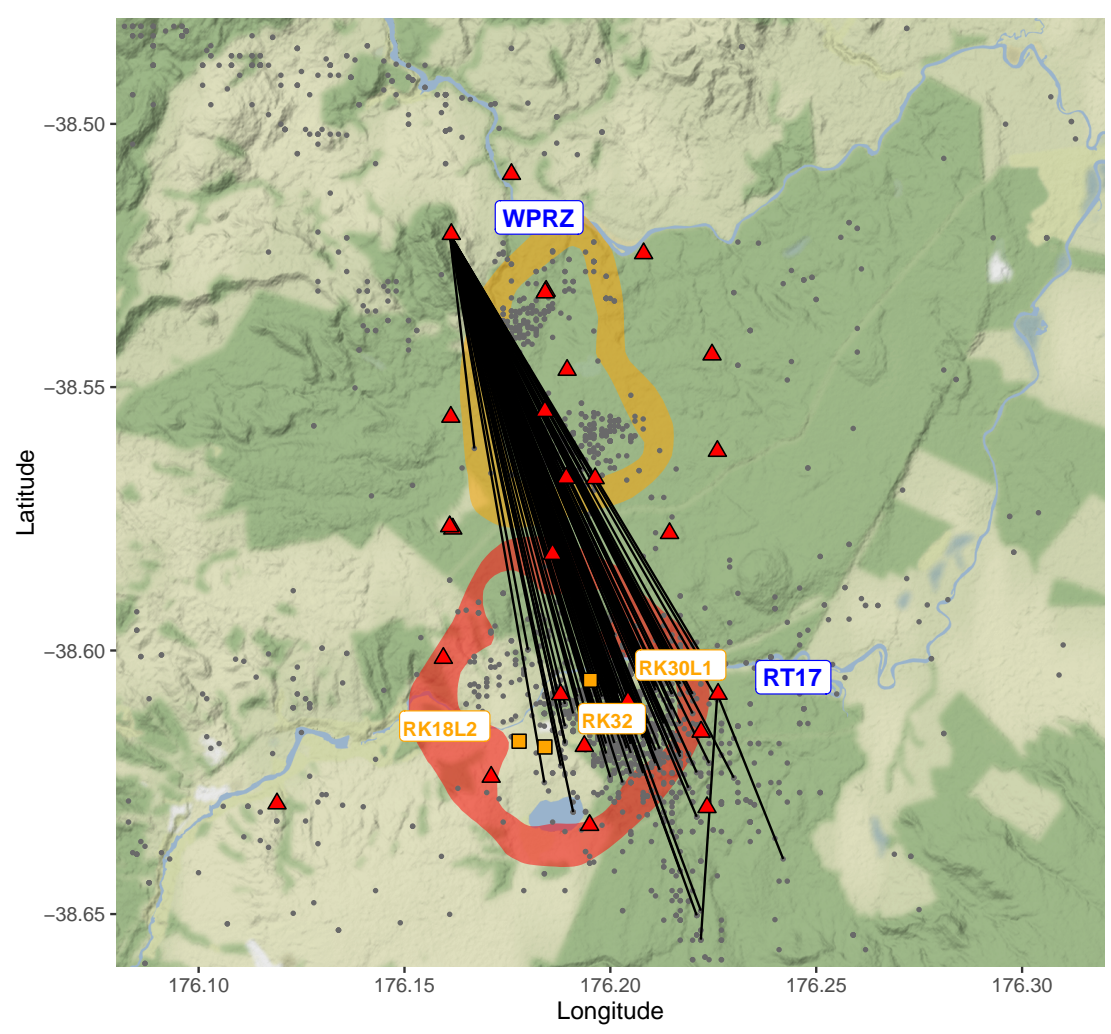

(c) Station-event paths for measurements in Group.

Figure 4.15: Summary of Group 7. Best fitting cracks striking 035․ 


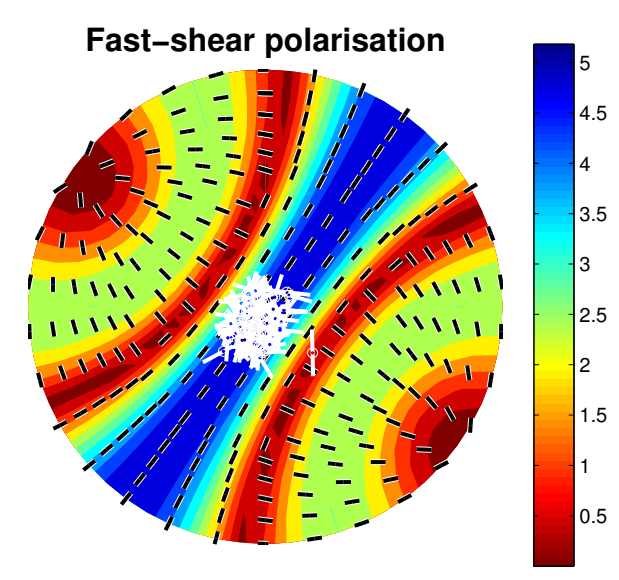

(a) Best fitting pole plot

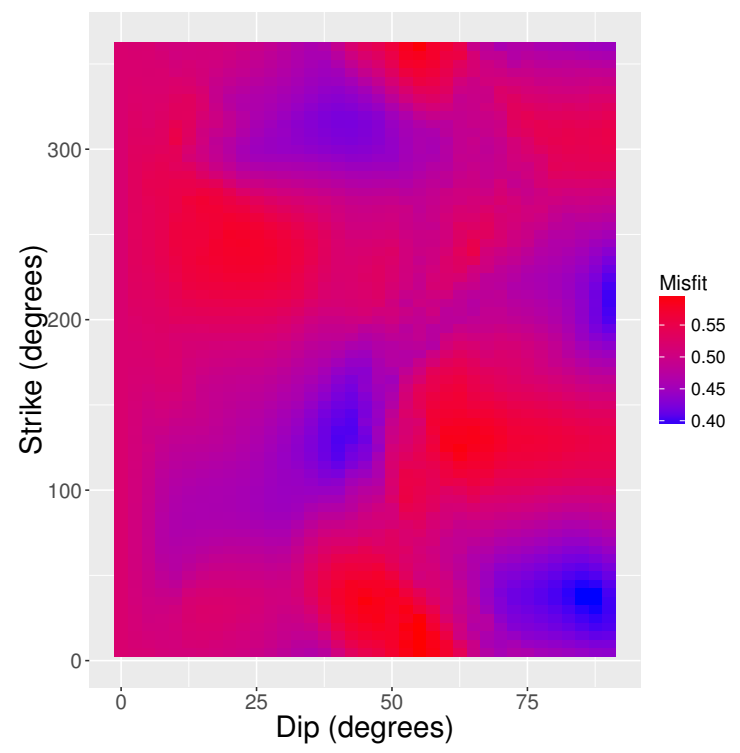

(b) Misfit grid

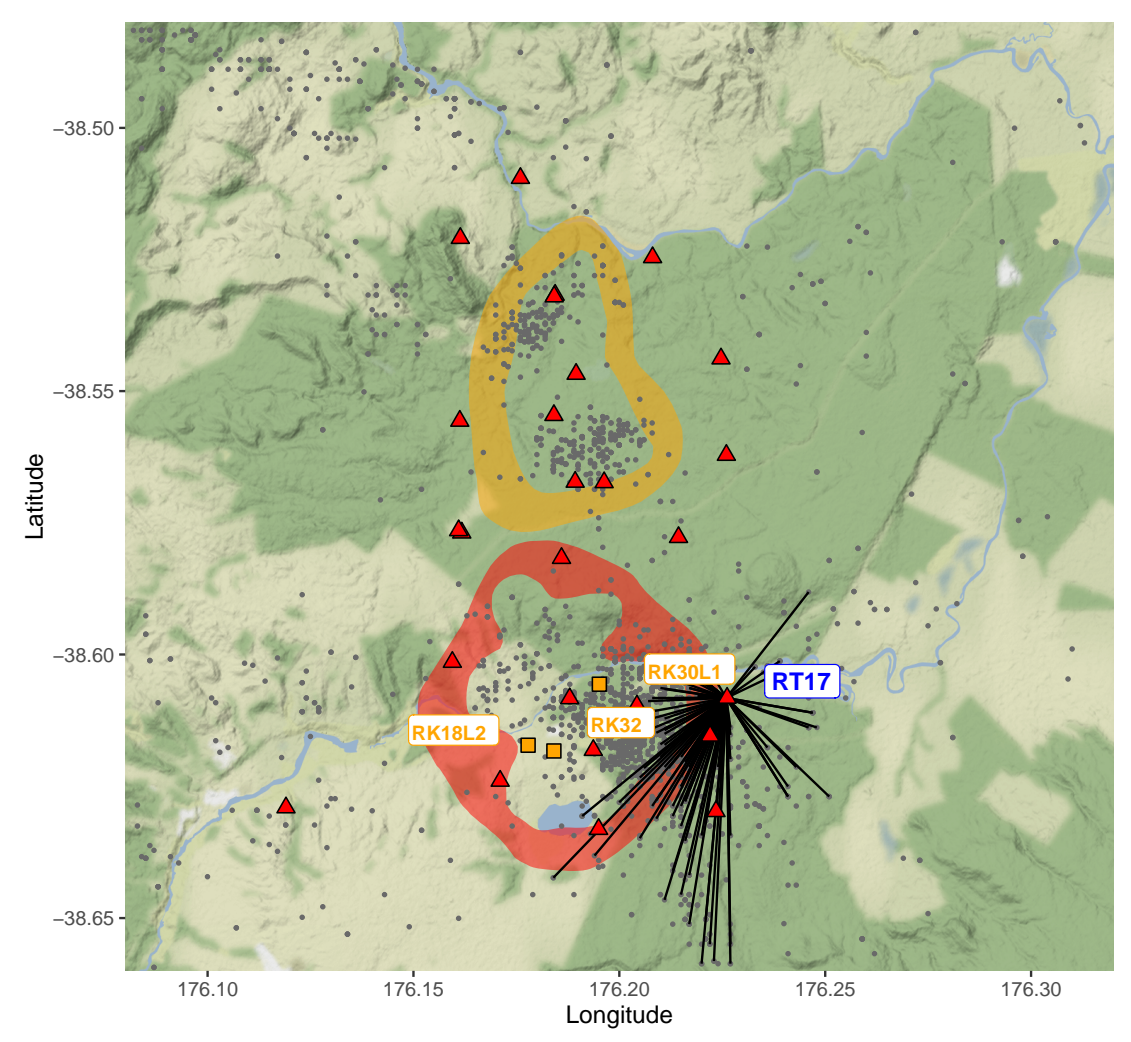

(c) Station-event paths for measurements in Group.

Figure 4.16: Summary of Group 38. Best fitting cracks striking 035. 


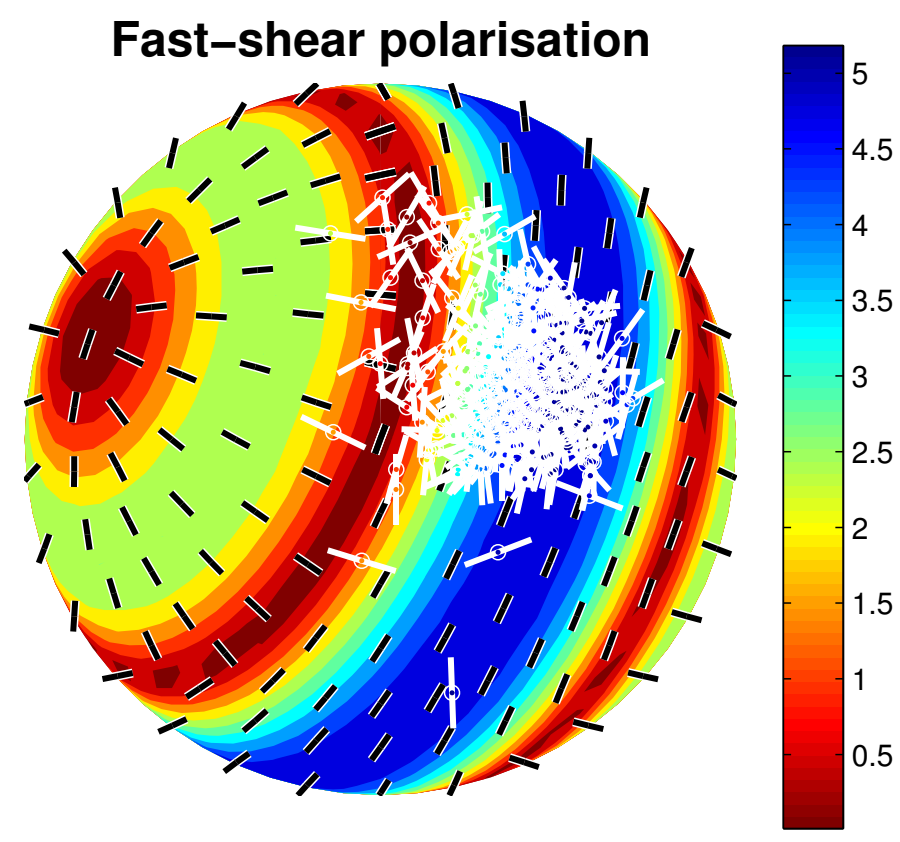

Figure 4.17: Spherical projection for best fitting crack plane of Group 25 with measured polarisations plotted in white. 


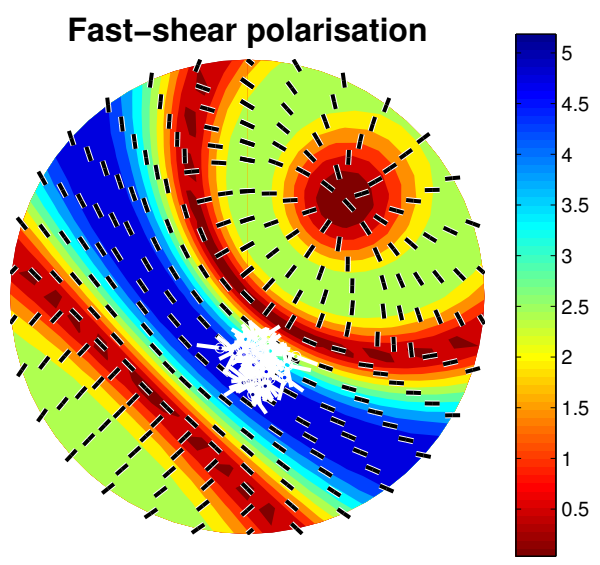

(a) Best fitting pole plot

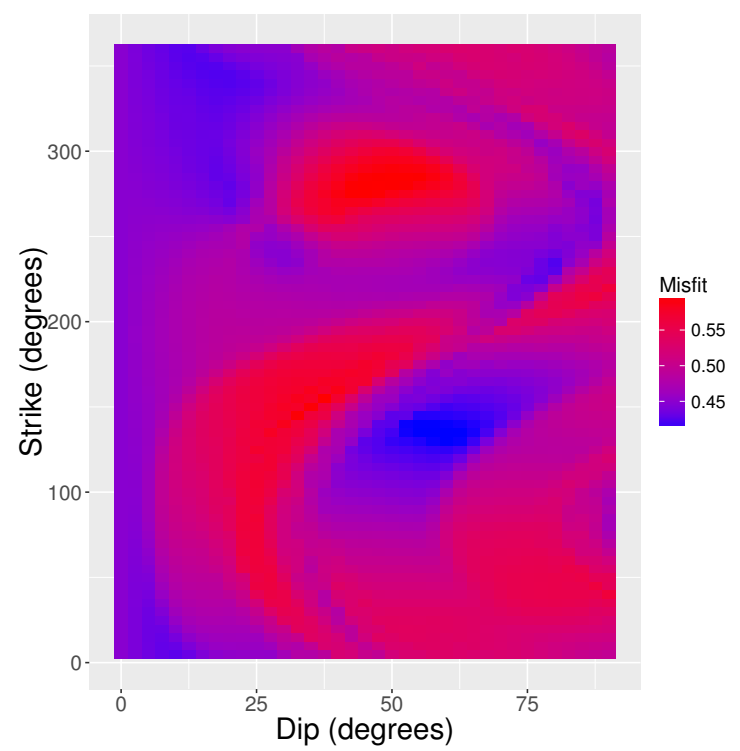

(b) Misfit grid

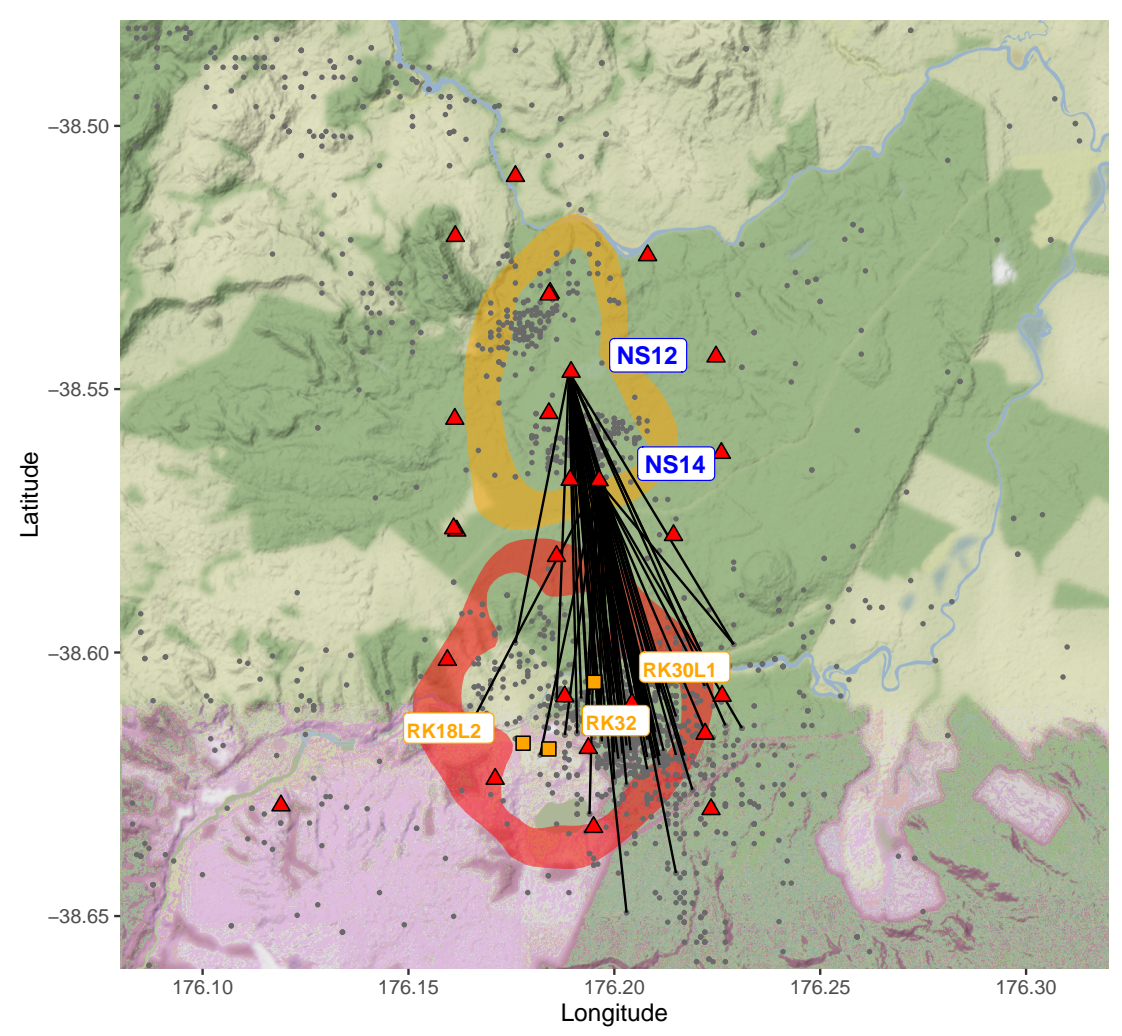

(c) Station-event paths for measurements in Group.

Figure 4.18: Summary of Group 8. Best fitting cracks striking -045․ 


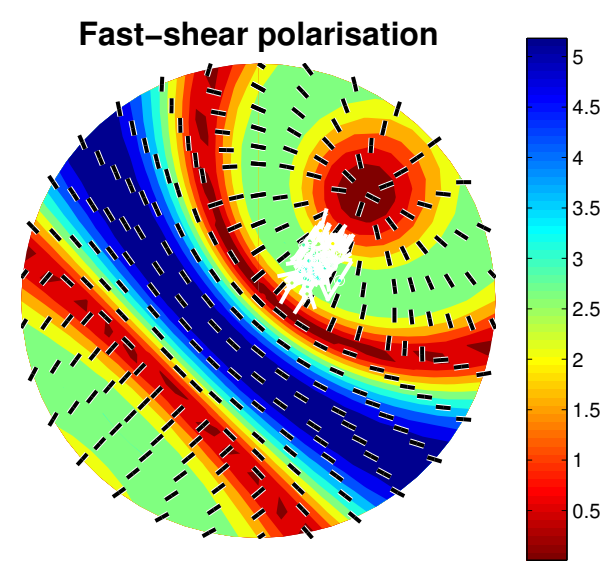

(a) Best fitting pole plot

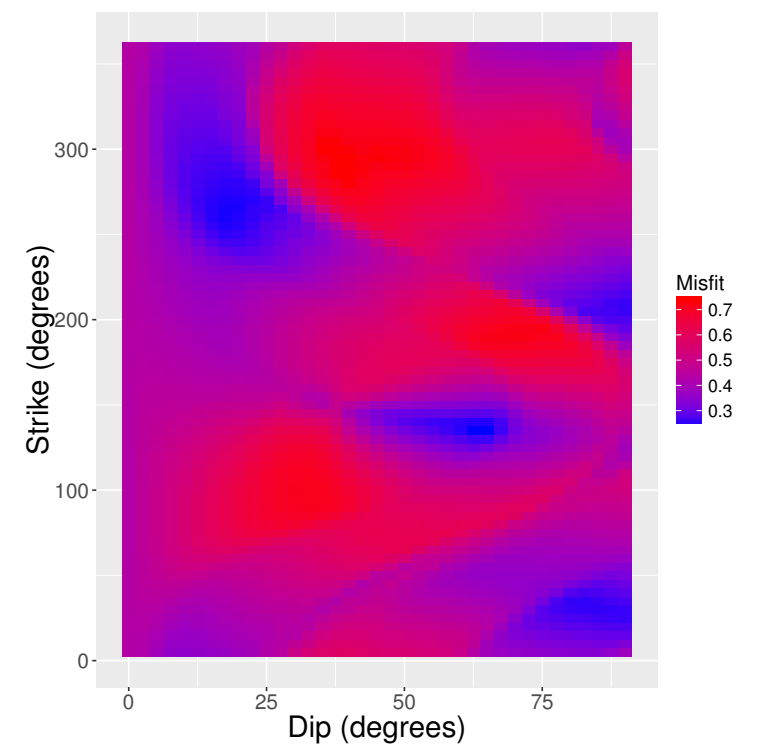

(b) Misfit grid

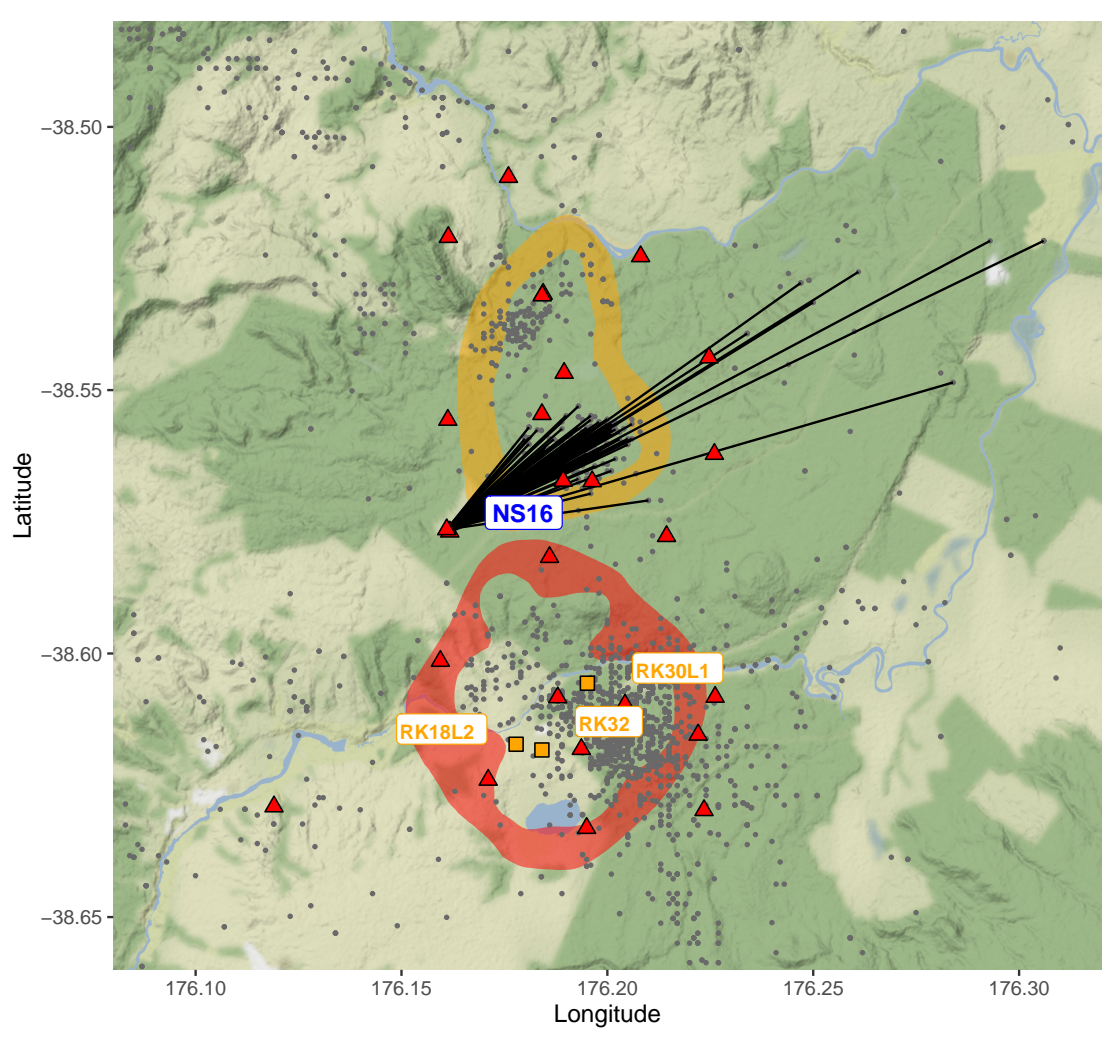

(c) Station-event paths for measurements in Group.

Figure 4.19: Summary of Group 18. Best fitting cracks striking-045․ 


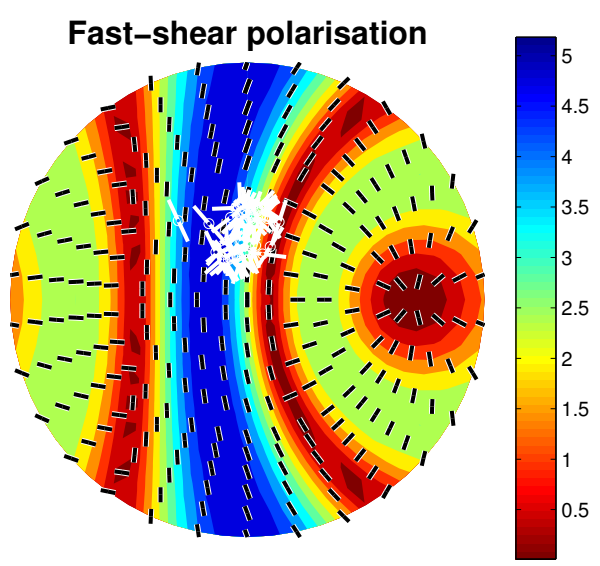

(a) Best fitting pole plot

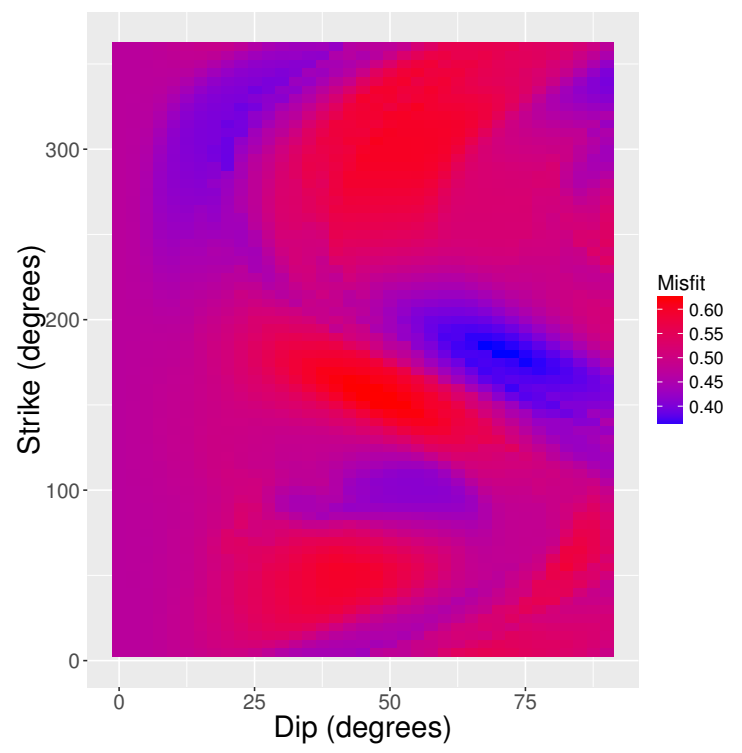

(b) Misfit grid

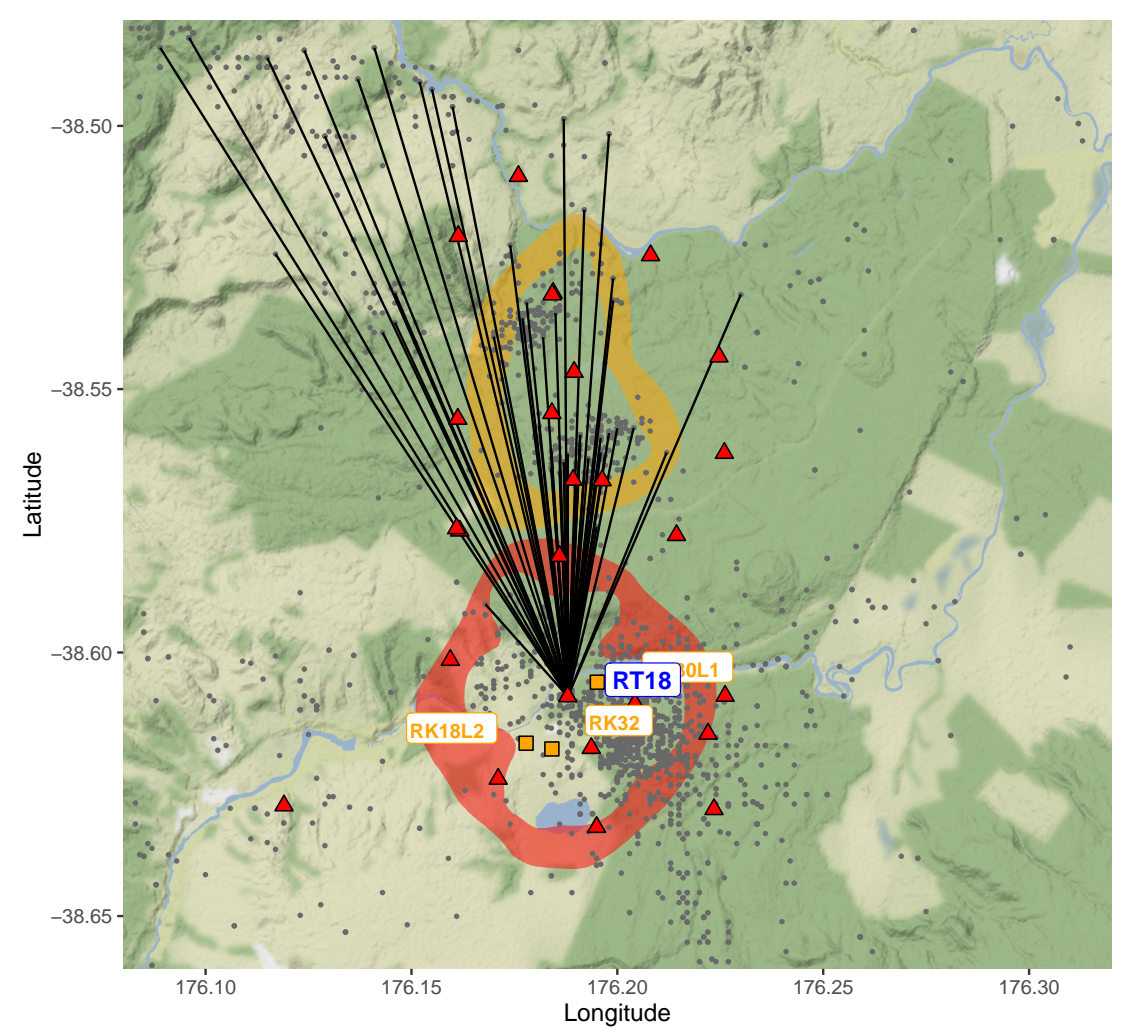

(c) Station-event paths for measurements in Group.

Figure 4.20: Summary of Group 14. Best fitting cracks striking $0^{\circ}$. 


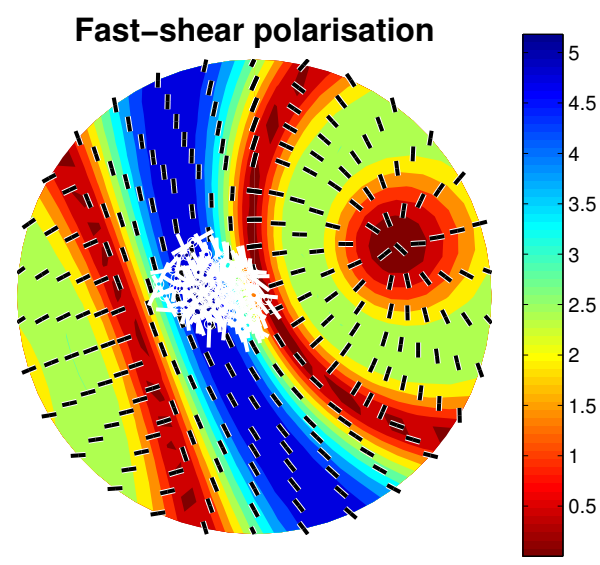

(a) Best fitting pole plot

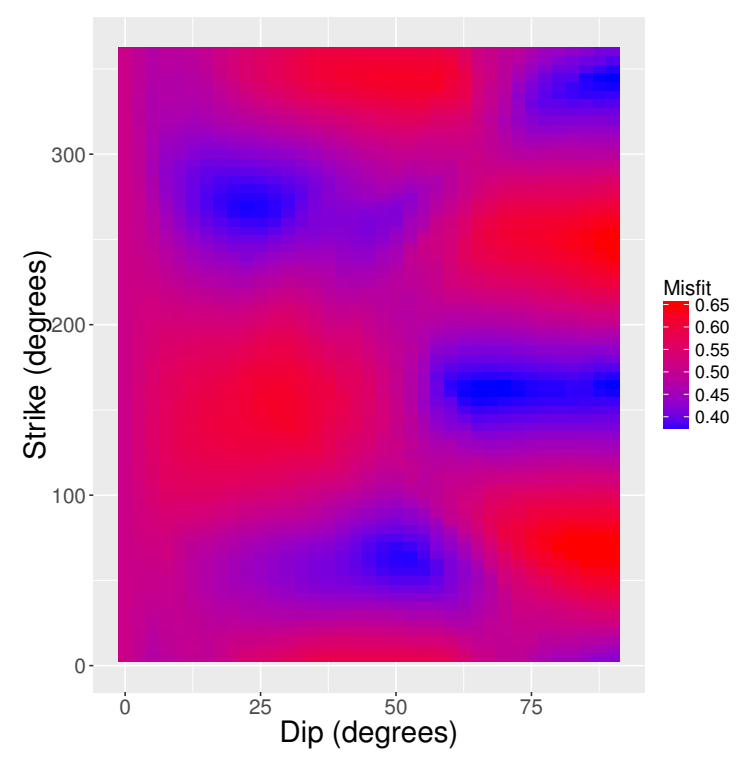

(b) Misfit grid

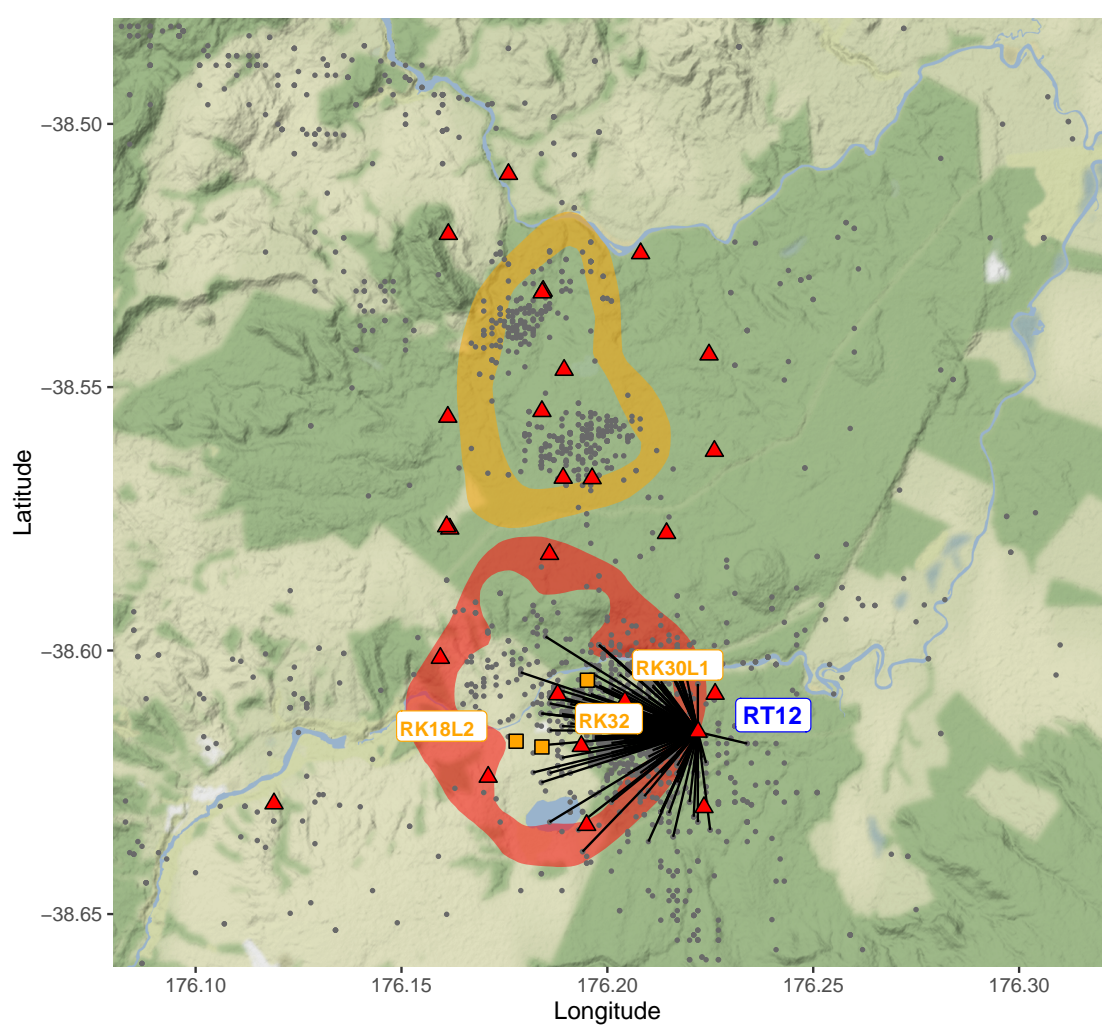

(c) Station-event paths for measurements in Group.

Figure 4.21: Summary of Group 34. Best fitting cracks striking-020 . 


\section{Groups with poor fit}

Figures not discussed at length in this section can be found in Appendix B.

Group 28 (Figure 4.22), with raypaths in south-eastern and central Rotokawa, has a best fitting crack strike of $080^{\circ}$. Group 43 (Figure 4.23), also with paths in central Rotokawa, has a best fitting crack plane which strikes $060^{\circ}$.

Group 3 and 29 (Figures B.4 \& B.5) should be treated with caution as both have low uniqueness for their fits (3rd and 2nd to last in uniqueness). Both groups have raypaths in central-southern Rotokawa. Group 29's crack plane is striking $010^{\circ}$, which is in the range of what we expect at Rotokawa based on Groups with better fits. Group 3 appears to be an outlier with a strike of $-040^{\circ}$.

Groups 26, 53, 37, and 40 (Figures B.1, B.2, B.3, and 4.24) have shallow dips (which we don't see for groups with high uniqueness) as well as low uniqueness and likely have not been fit correctly. As with other groups which do not have good fits, it is probable that errors in earthquake location, velocity model and local changes in stress are causing errors in the fit. If the majority of measurements in that group do not correspond to a single crack plane (or several similar crack planes) the fit will not be good.

Group 31 (Figure B.6) has the lowest uniqueness of all Groups containing above 50 measurements. With a strike of $-060^{\circ}$ it appears to be incorrectly fit as the majority of its measurements have a polarisation of $\sim 045^{\circ}$.

\section{Comparison with borehole measurements}

Most groups (with high uniqueness) have compatible (restricted) polarisations with in-situ stress measurements from at least one well (summarised in Table 4.4) based on Watson's test (Section 3.2.4). Very local changes in stress are present in the Rotokawa reservoir with orientation changes between wells (described in Section 1.4.2) on scales of at least $020^{\circ}$. The raypaths for each of our groups cover a much 
wider area than even two of these boreholes and so we can expect significant scatter in our polarisations for each group.

$56 \%$ of the groups, that had enough restricted measurements to perform Watson's test, had means which did not appear to differ from the mean of at least one of the wells' in-situ stress measurements. Thus, our fitted crack planes appear to be compatible with in-situ measurements of the direction of $S_{H_{\max }}$

\section{Stations close to Rotokawa seismicity}

Fast polarisations at stations RT14, RT18 and RT19 fail the Rayleigh test and we inferred that this may be due to their location with respect to the majority of the seismicity (Section 4.3.4, Figure 4.5). Groups containing RT19 did not make the 50 measurement threshold for the fitting process and thus are not shown (this station only has small amount of shear wave splitting measurements). RT18 has a significant amount of measurements (263) and is represented in multiple groups (Group 14, Group 43 and Group 40, RT14 is also contained in Group 28).

Group 14 (Figure 4.20) contains solely measurements from RT18 and, with a crack plane striking $0^{\circ}$, is within the range we expect at Rotokawa. Events in this group are located in Ngatamariki so back azimuths are all similar.

Group 40 (Figure 4.24) also solely contains events measured on RT18, however, the best fitting crack plane has an unreasonably shallow dip. With its low uniqueness, this indicates that the second best local minimum with cracks striking approximately $0^{\circ}$ is closer to what we expect at Rotokawa.

Both Group 28 (Figure 4.22) and Group 18 (Figure 4.19) are interesting as they contain steep angles of incidence (with a wide range of back azimuths) on stations RT14 and RT18 respectively. Inspection of the misfit grids of these groups reveals that they are fairly similar, with dip being more well constrained than strike. Given this feature, it is likely that propagation angles are too different to get a good fit 
with a single crack plane.

The fact that the crack fitting process has successfully gained information from both these stations (which otherwise had no evidence of having a dominant mean orientation) shows that the crack fitting process is giving desirable results.

\section{Improvements}

The crack fitting process would be improved by more exact knowledge of the propagation angle. This requires precise velocity in both Rotokawa and Ngatamariki and well located earthquakes (which requires an accurate velocity model).

More specific estimation of the effective propagation angle (the propagation angle best approximating the single anisotropic layer assumption) would also be required (as propagation angle varies along the path).

\subsubsection{Fitting with uniform velocity model}

Fitting our measurements using angles of propagation esimated from angles of incidence at sea level determined from a Rotokawa velocity model (Section 3.6.2) represents an end member of the effective propagation angle for our measurements (Section 3.8.3). We also test the other end member of a uniform velocity model (straight line propagation angle) here. For our groups with propagation angles determined from the Rotokawa velocity model, we were able to apply three iterations of hierarchical clustering (used to group similar groups of measurements together). For our groups determined with a uniform velocity model, a third pass of hierarchical clustering could not be applied as no groups of measurements had high enough similarity.

Table 4.5 gives all the groups from the crack fitting when estimating propagation angles from a uniform velocity model. We do not plot these except for Group 39 which has the highest uniqueness.

Figure 4.25 shows the summary of the group with the highest uniqueness. Because 


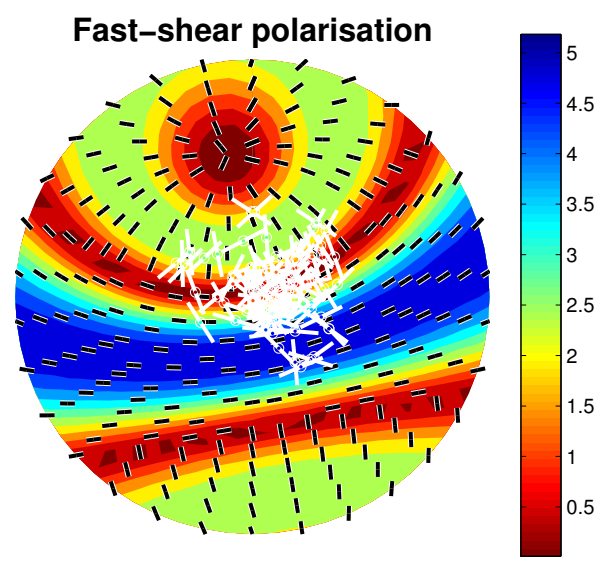

(a) Best fitting pole plot

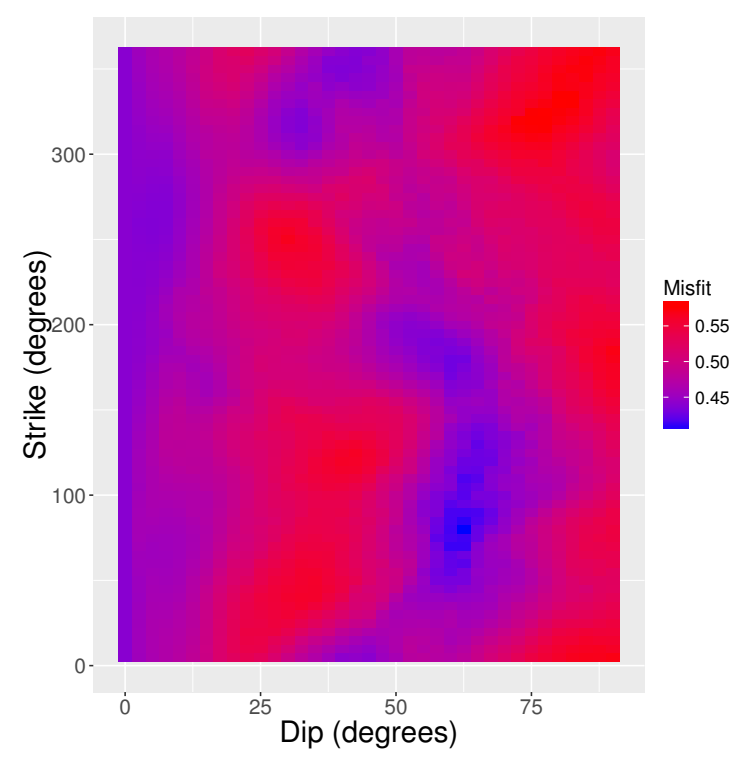

(b) Misfit grid

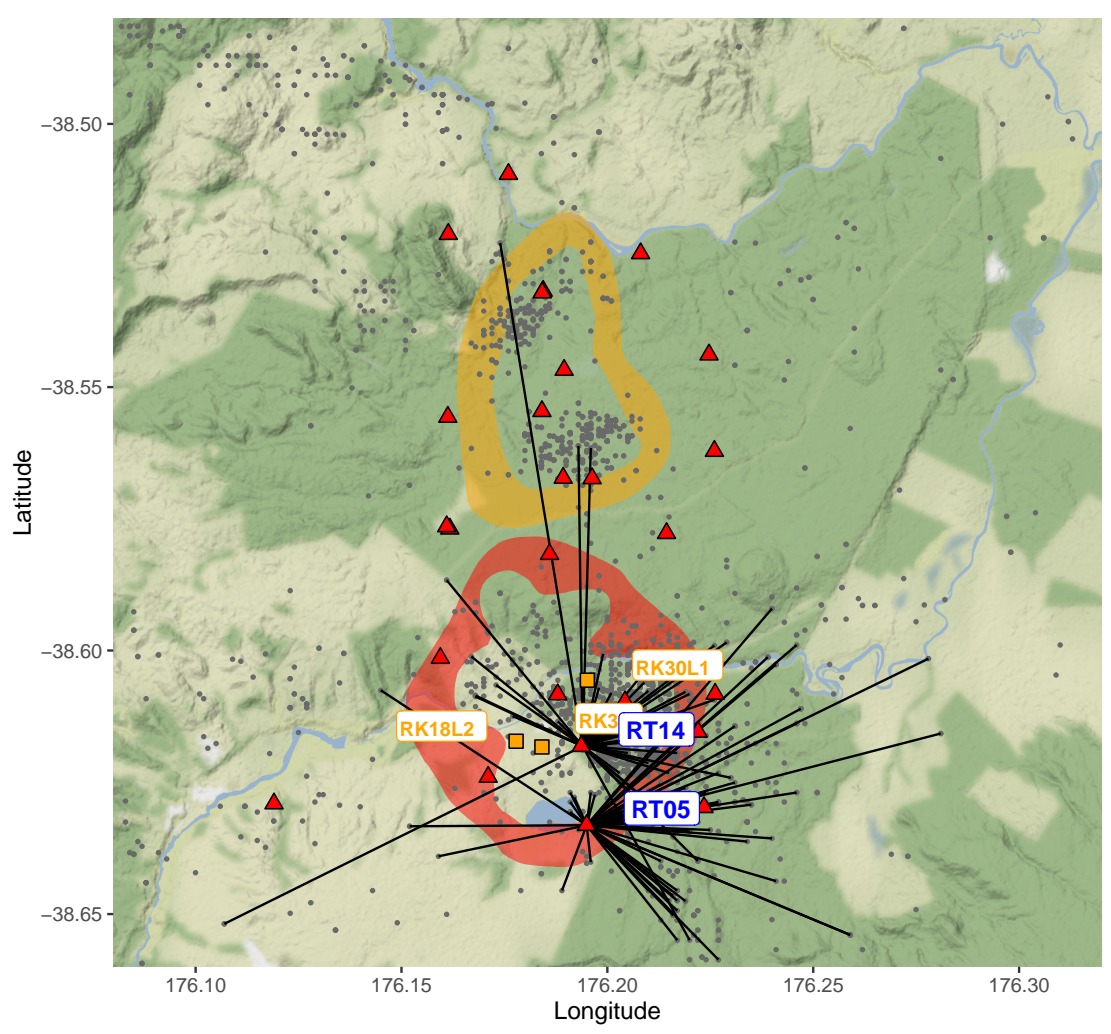

(c) Station-event paths for measurements in Group.

Figure 4.22: Summary of Group 28. Best fitting cracks striking $080^{\circ}$. 


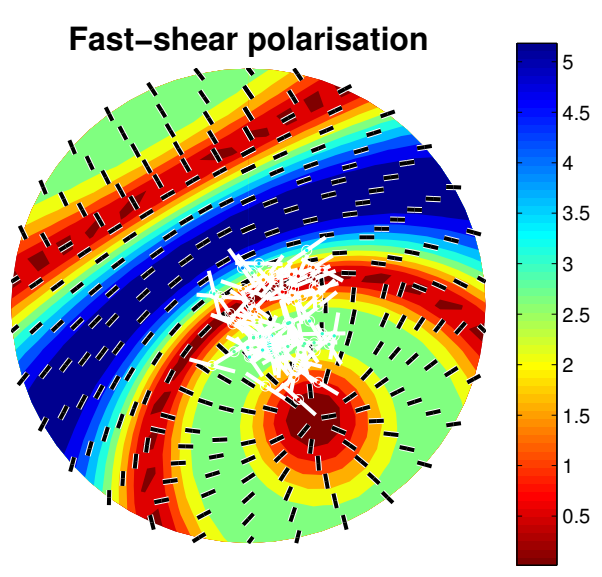

(a) Best fitting pole plot

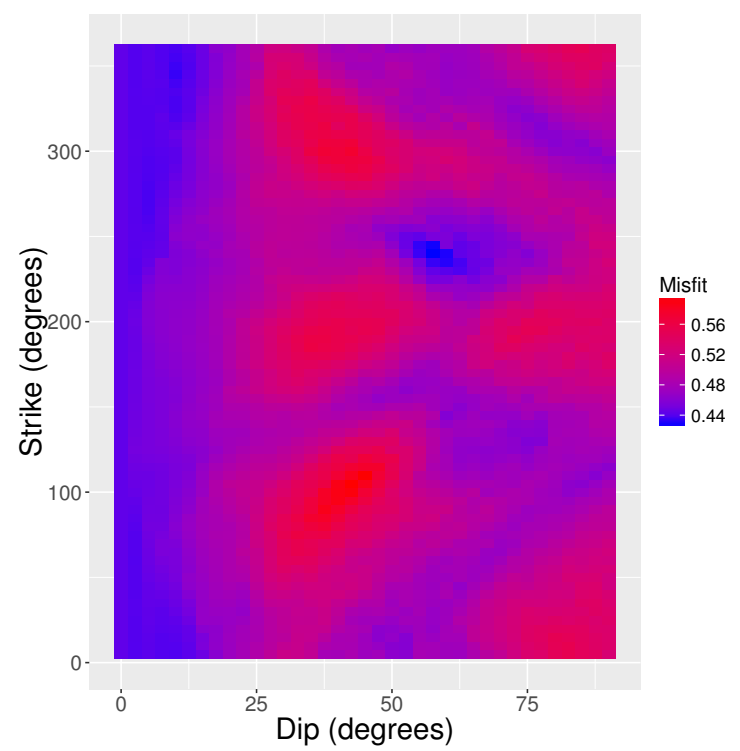

(b) Misfit grid

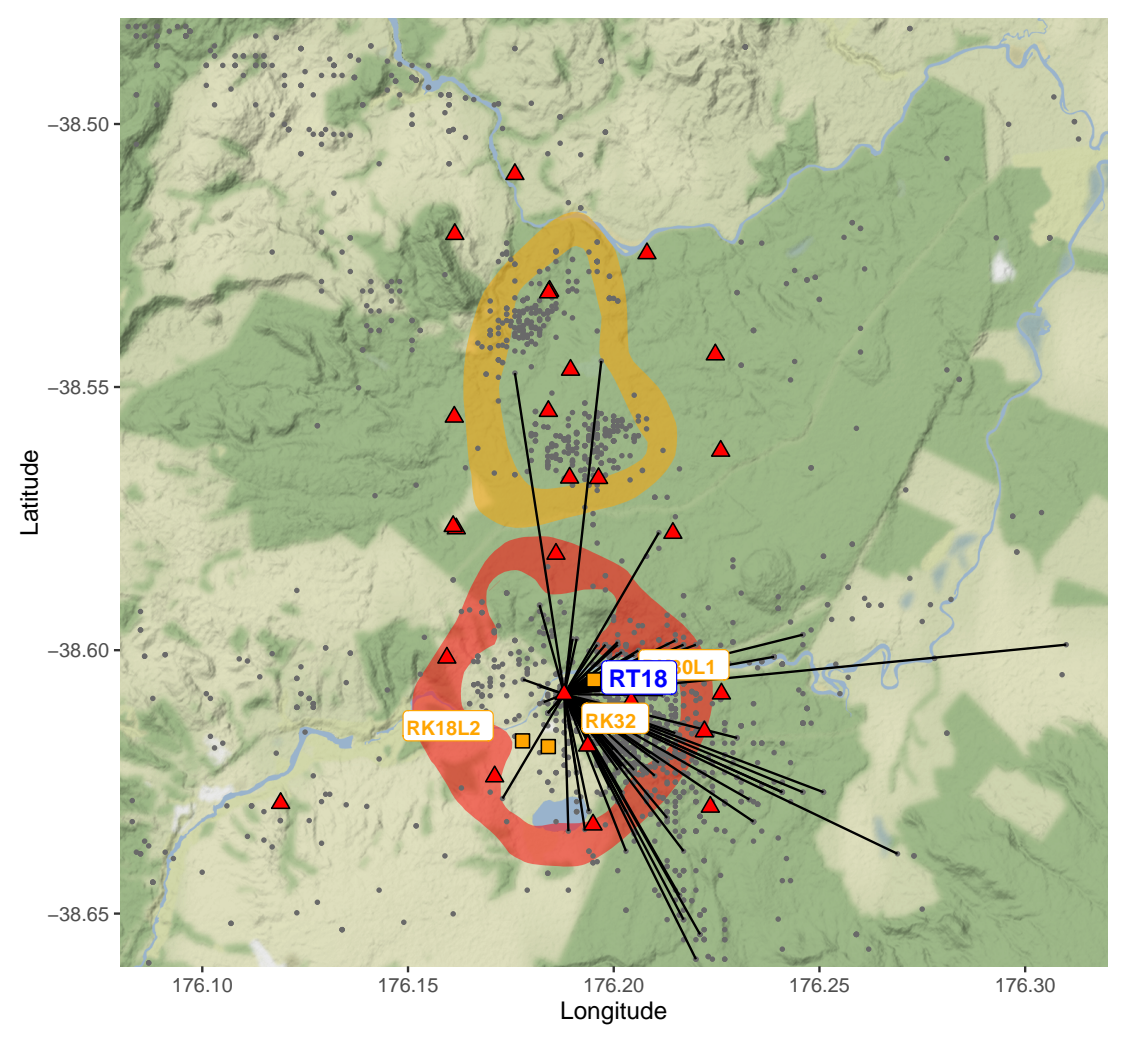

(c) Station-event paths for measurements in Group.

Figure 4.23: Summary of Group 43. Best fitting cracks striking $060^{\circ}$. 


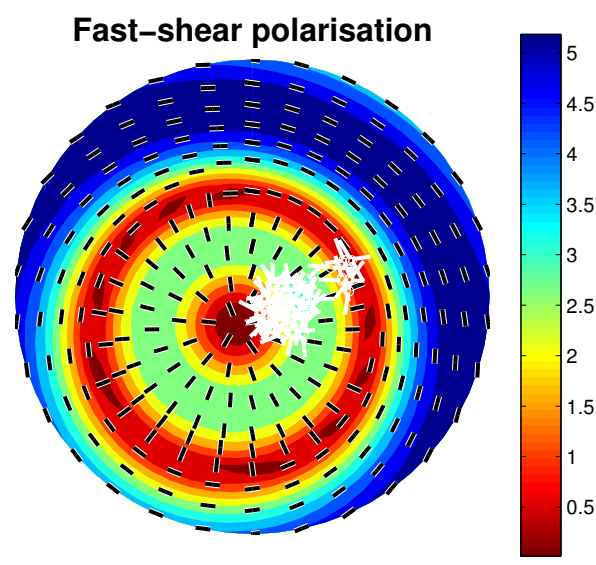

(a) Best fitting pole plot

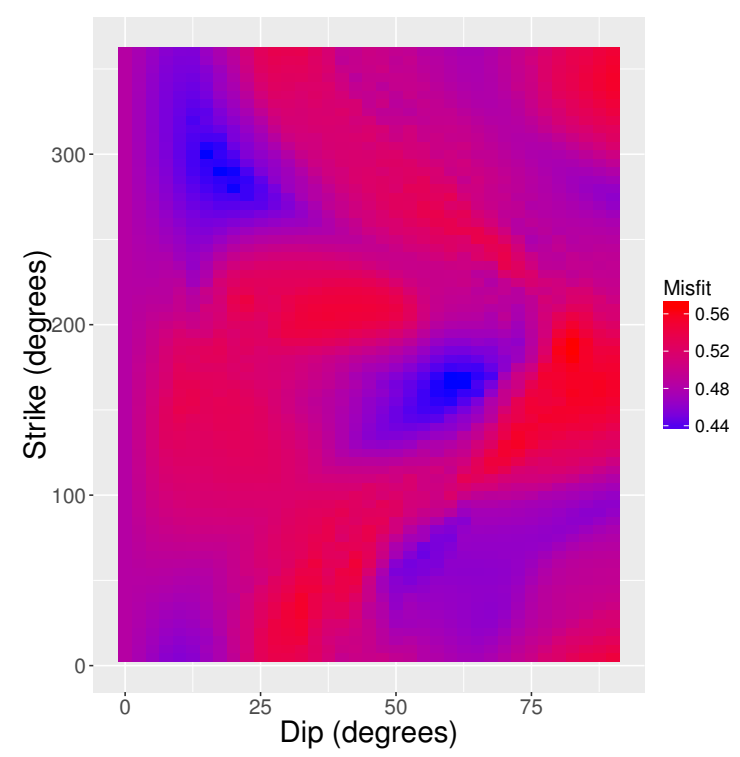

(b) Misfit grid

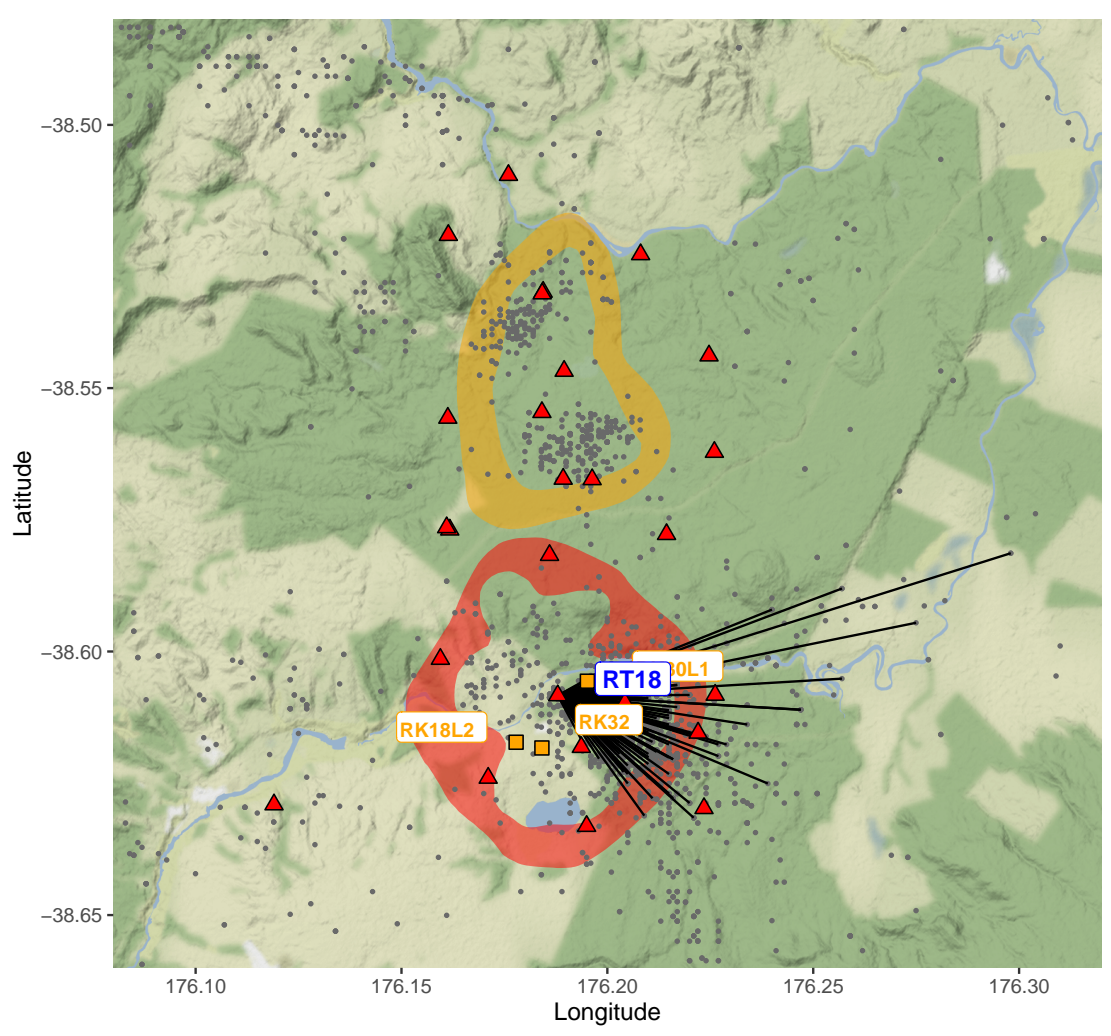

(c) Station-event paths for measurements in Group.

Figure 4.24: Summary of Group 40. Best fitting cracks striking-060'. 
events are all close to the station, most measurements are grouped in a central area. As a result, the straight line path does not differ much from the angle of incidence determined from the more accurate velocity model (Section 3.6.2).

Overall, the fit from straight line propagation angles is worse both in uniqueness and misfit. Those which do show high uniqueness, such Group 39 (straight line), are those which are closest to sub-vertical propagation angles. Straight line propagation angles almost certainly overestimate the effective propagation angle however to what degree they do so is what influences the final fit. We assume anisotropy is measured closer to the station (Rümpker and Silver 2000; Nistala and McMechan 2005). Also, if propagation angle moves from shallow to sub-vertical along the raypath then the fast polarised wave will become the slow polarised wave and vice versa (Section 3.8.3). Depending how early along the raypath this occured, the two waves can switch positions and thus, at the station, a fast polarisation parallel with the crack strike will be measured but with an anomalously low delay time. We do have some evidence for this occurring (Section 3.8.3). 


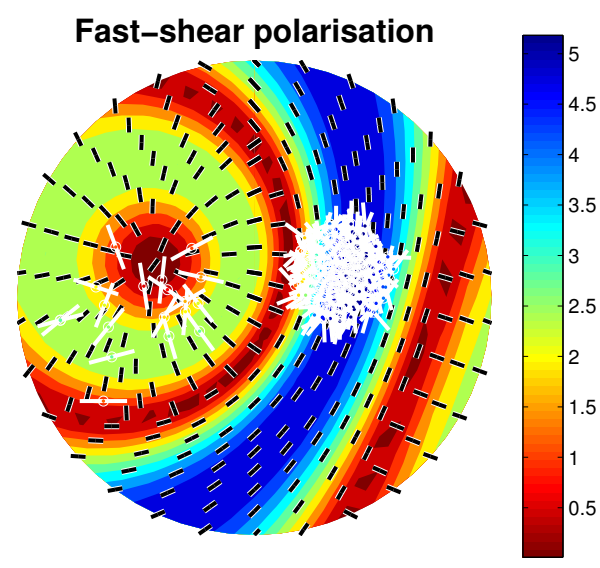

(a) Best fitting pole plot

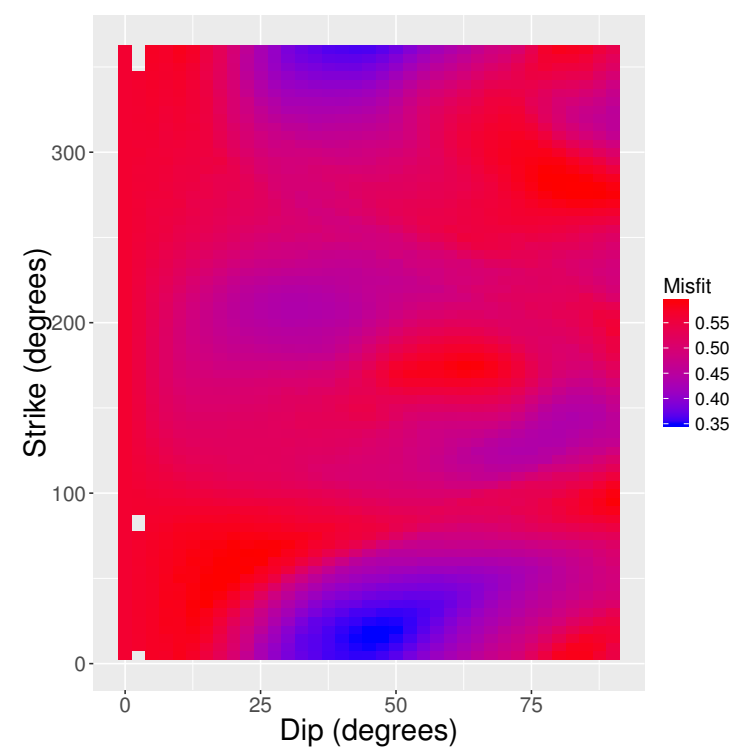

(b) Misfit grid

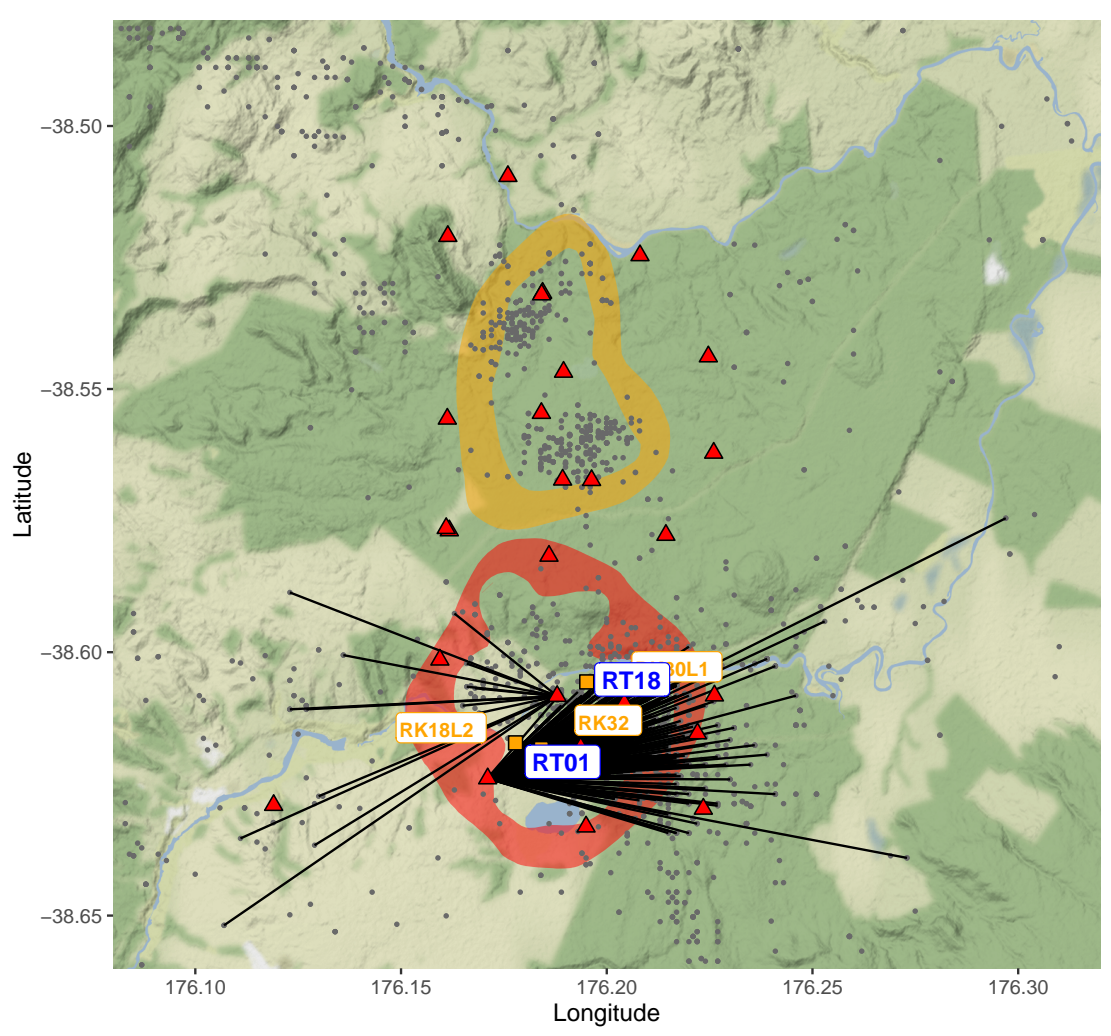

(c) Station-event paths for measurements in Group.

Figure 4.25: Summary of Group 39 for fitting with straight line propagation angle. Best fitting cracks striking 020 . 
Table 4.5: Table of fitted crack planes determined with straight line propagation angles. For columns labelled with well name, "Yes" indicates no significant difference in mean between restricted polarisations and $S_{H_{\max }}$ measured in that well ( $p$-value $>$ 0.05). NA indicates that either there weren't enough restricted measurements to do the comparison or the restricted measurements do not have a dominant mean orientation (fail the Rayleigh test). Mean delay times of the (restricted) groups are also quoted $(\delta t)$.

\begin{tabular}{|c|c|c|c|c|c|c|c|c|}
\hline & Strike $\left(^{\circ}\right)$ & $\operatorname{Dip}\left(^{\circ}\right)$ & $\begin{array}{c}\text { Dip } \\
\text { Azimuth }\left(^{\circ}\right)\end{array}$ & $\delta t$ & RK18L2 & RK32 & RK30L1 & $\#$ \\
\hline Group39 & 20 & 47.5 & 290 & 0.095 & No & No & No & 310 \\
\hline Group36 & 35 & 60 & 305 & 0.087 & NA & NA & NA & 90 \\
\hline Group31 & 40 & 35 & 130 & 0.097 & Yes & No & No & 97 \\
\hline Group30 & 35 & 80 & 305 & 0.048 & No & No & No & 83 \\
\hline Group4 & 20 & 55 & 290 & 0.097 & Yes & No & No & 69 \\
\hline Group48 & -20 & 70 & 70 & 0.104 & No & No & No & 120 \\
\hline Group25 & -85 & 77.5 & 185 & 0.092 & NA & NA & NA & 54 \\
\hline Group50 & -25 & 62.5 & 245 & 0.092 & NA & NA & NA & 92 \\
\hline Group51 & 40 & 77.5 & 310 & 0.1 & Yes & Yes & No & 98 \\
\hline Group40 & 55 & 62.5 & 325 & 0.077 & NA & NA & NA & 69 \\
\hline Group5 & 40 & 60 & 130 & 0.11 & Yes & No & No & 62 \\
\hline Group41 & -40 & 70 & 50 & 0.092 & NA & NA & NA & 206 \\
\hline Group45 & 55 & 72.5 & 325 & 0.092 & NA & NA & NA & 59 \\
\hline Group53 & 50 & 40 & 320 & 0.089 & NA & NA & NA & 99 \\
\hline Group43 & 90 & 85 & 360 & 0.092 & NA & NA & NA & 66 \\
\hline Group49 & -25 & 60 & 65 & 0.099 & NA & NA & NA & 54 \\
\hline Group42 & 20 & 62.5 & 290 & 0.069 & NA & NA & NA & 129 \\
\hline Group2 & 50 & 60 & 140 & 0.092 & NA & NA & NA & 191 \\
\hline Group55 & -55 & 65 & 35 & 0.07 & NA & NA & NA & 81 \\
\hline
\end{tabular}




\subsection{TESSA}

Delay time tomography and spatial averaging of fast polarisations using, the TESSA package, works on the assumption that all angles of propagation are sub-vertical and that the cracks are vertical. By fitting crack planes we can sanitize the inputs for TESSA to only allow shear wave splitting measurements which theoretically should all be aligned with $S_{H_{\max }} /$ crack strike as well as having delay times which stay constant with propagation angle. This is described further in Section 3.9.1.

This averaging and tomography is a first order approximation to the actual anisotropy (Johnson et al. 2011). It provides a good way of visualising the average anisotropy for the entire field in only two figures. For delay time tomography, its strength lies in highlighting regions of higher anisotropy rather than providing accurate estimates of what the strength of anisotropy is.

It should be noted that the background anisotropy across the fields is high. For delay time tomography carried out by Johnson et al. (2011) around Mt Ruapehu (located in the southern TVZ) maximum anisotropy (over a much much larger study area) is $0.025 \mathrm{~s} / \mathrm{km}$. Whereas, for Figure 4.28 the maximum anisotropy is $\sim 0.13$ $\mathrm{s} / \mathrm{km}$. To plot delay time tomography results we use a linear interpolation to convert the results from an irregular grid to a surface (Akima and Gebhardt 2015).

Spatial averaging means are determined from polarisations weighted by $1 / d^{2}$, where $d$ is the distance of the grid block from the station. Blocks/rose plots whose (weighted) polarisations have a standard error greater than $10^{\circ}$ or circular standard deviation greater than $30^{\circ}$ are removed as they exhibit high scatter or more than one mode (Johnson et al. 2011). 


\subsubsection{Full dataset}

\section{Spatial averaging}

Figure 4.26 shows the spatially averaged shear wave splitting fast polarisations $(\phi)$, across both Rotokawa and Ngatamariki, for the full dataset. Raypaths and quadtree grid are plotted in Figure 4.30a.

We see N-S average orientations in eastern Rotokawa which rotate through to NE-SW orientations in south-eastern Rotokawa. Eastern Rotokawa has average orientations rotating from NNW-SSE in the south through to NE-SW in the north.

Northern and western Ngatamariki have orientations fairly uniformly in a NESW orientation (with regional $S_{H_{\max }}$ ). In central-southern Ngatamariki there is significant rotation away from NE-SW. This may be, in part, due to the addition of borehole stations (which may be returning different polarisations from surface stations). A denser station spacing in the area may be illuminating more local changes in stress or the stress field may be being perturbed by the extraction or injection of fluids.

Mean orientations align closely to the strike of the faults. This is what we expect for a normal faulting regime such as the TVZ, where $S_{H_{\text {max }}}$ is parallel to fault strike. This is especially apparent in Rotokawa (Figure 4.26) where the mean fast polarisations align closely with the central field fault and injection field fault to the east and then rotate more towards N-S to align with the production field fault (PFF).

On the eastern boundary Rotokawa (Figure 4.26), there appears to be a rotation of the polarisations toward NNW-SSE and this is measured on more than one station in the area. This could indicate a different source of anisotropy other than stress aligned microcracks. 


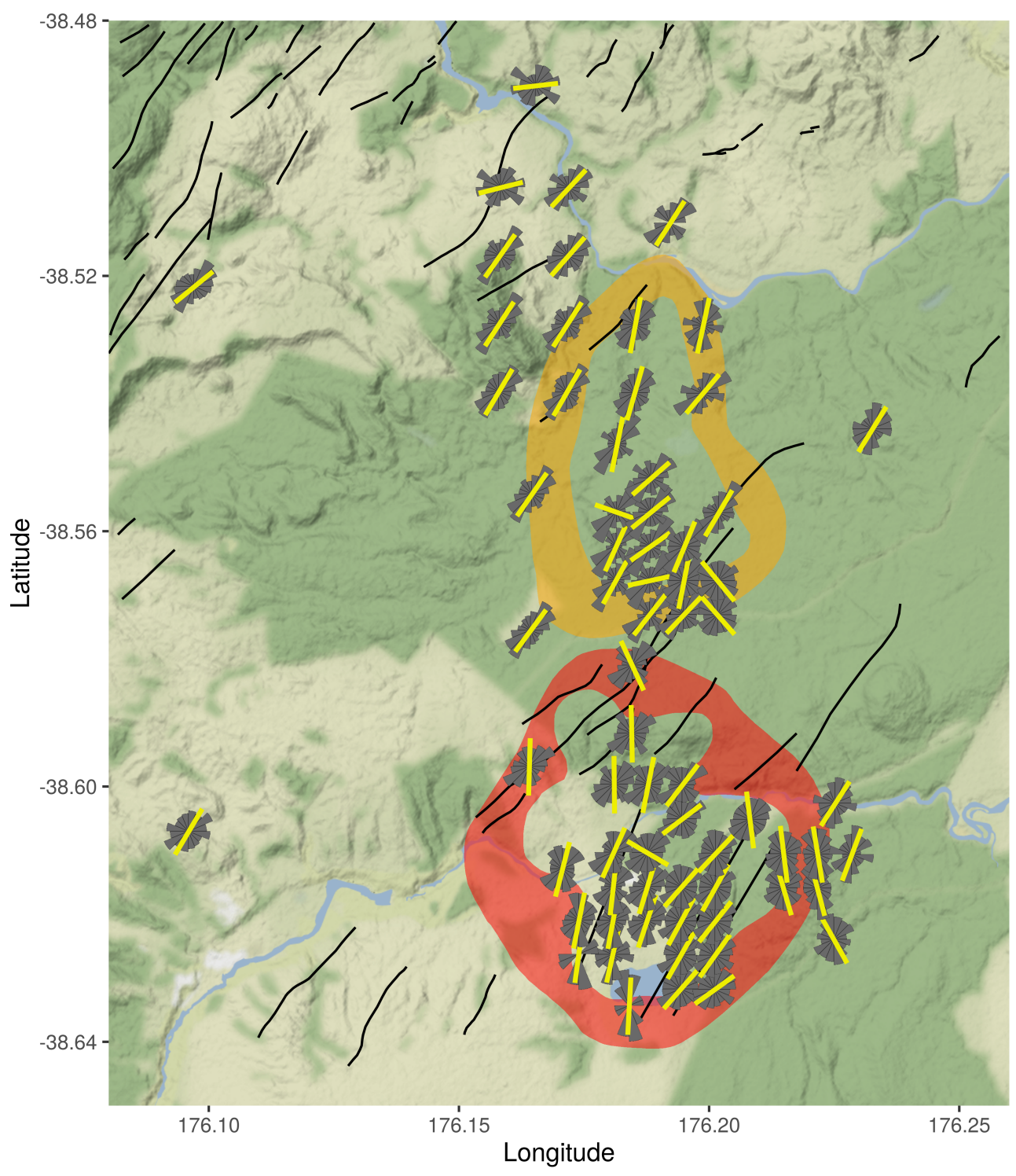

Figure 4.26: Spatial average of shear wave splitting fast polarisations ( $\phi$ ) for the full dataset. Determined with $1 / d^{2}$ weighting and quadtree gridding using the TESSA package. Rose plots with high standard error or standard deviation are not included. Resistivity boundaries for Rotokawa (south) and Ngatamariki (north). Black lines represent fault traces. 


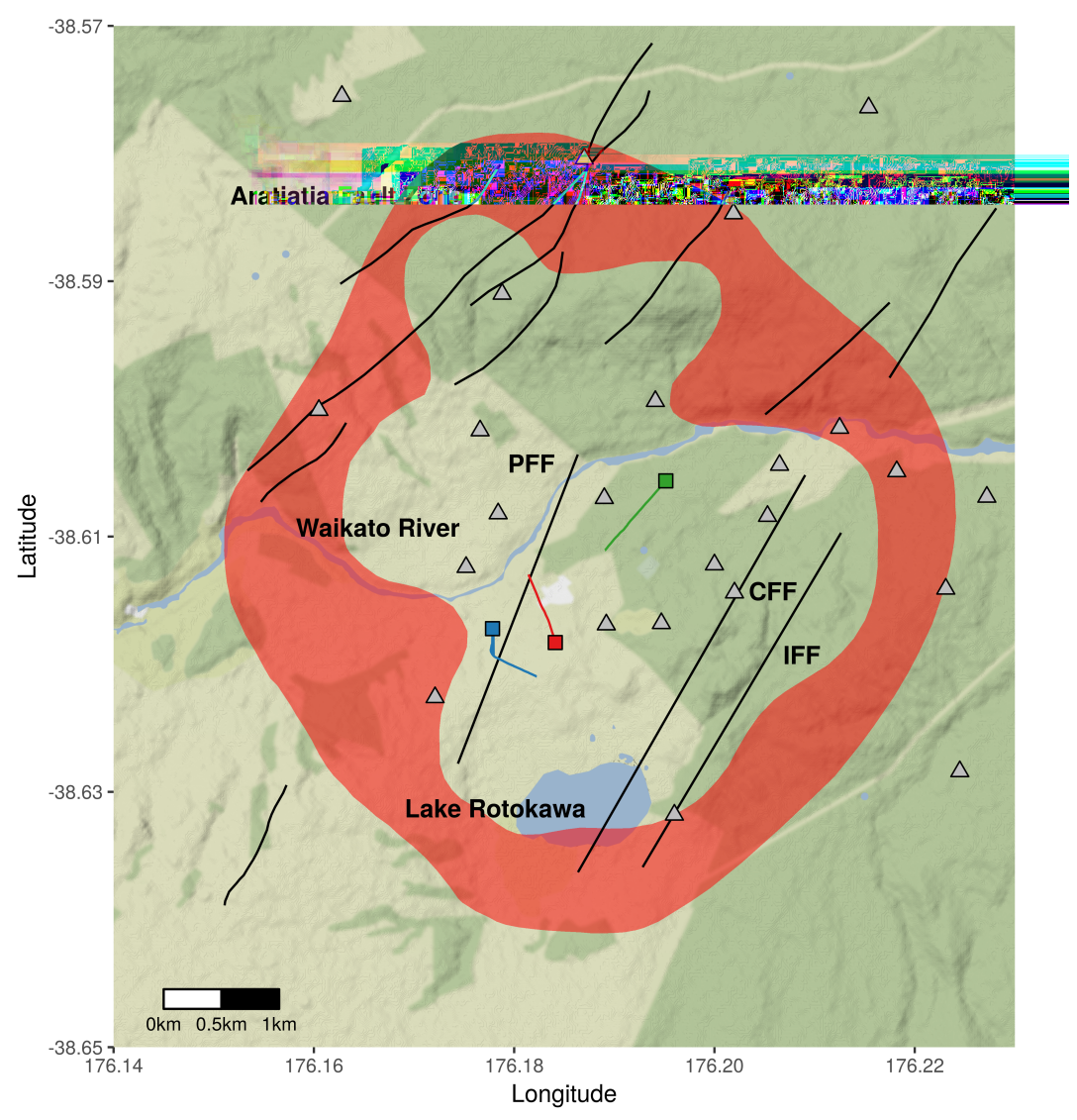

Figure 4.27: Spatial average of shear wave splitting fast polarisations ( $\phi$ ) for the full dataset. Zoomed to Rotokawa to highlight relationship between polarisations and the production field fault (PFF), central field fault (CFF), and injection field fault $(I F F)$. 


\section{Delay time tomography}

Figure 4.28 shows the delay time tomography for the full dataset. Checkerboard test and resolution are shown in Figure 4.29 and 4.30.

The largest anisotropy appears to be in southern-central and north-eastern Rotokawa. Two small regions of higher anisotropy appear on the production field fault $(\mathrm{PFF})$ and may be related to it. The injection field fault (IFF) also appears like it may be associated with some higher anisotropy. A moderately high amount of anisotropy is present in northern Ngatamariki with a smaller amount in centralsouthern Ngatamariki.

As expected, the resolution is best within Rotokawa however still only vaguely returns the checkerboard. Regions of high anisotropy are over estimated so it is likely the localised peaks of high anisotropy seen in Figure 4.28 are artefacts and in reality are smoother.

\subsubsection{Restricted dataset}

Both spatial averaging and delay tomography suffered from a lack of measurements in the restricted dataset (2944 in the full dataset to 664 in the restricted dataset). This situation would likely be improved if the velocity model was better known as this would improve estimates of propagation angle. The addition of more years of data, if changes in anisotropy through time are assumed to be small, would also improve the results by increasing the number of events in each group/cluster of measurements.

\section{Spatial averaging}

Figure 4.31 shows the spatially averaged shear wave splitting fast polarisations $(\phi)$, across both Rotokawa and Ngatamariki, for the restricted dataset. Raypaths and quadtree grid are plotted in Figure 4.34a. 


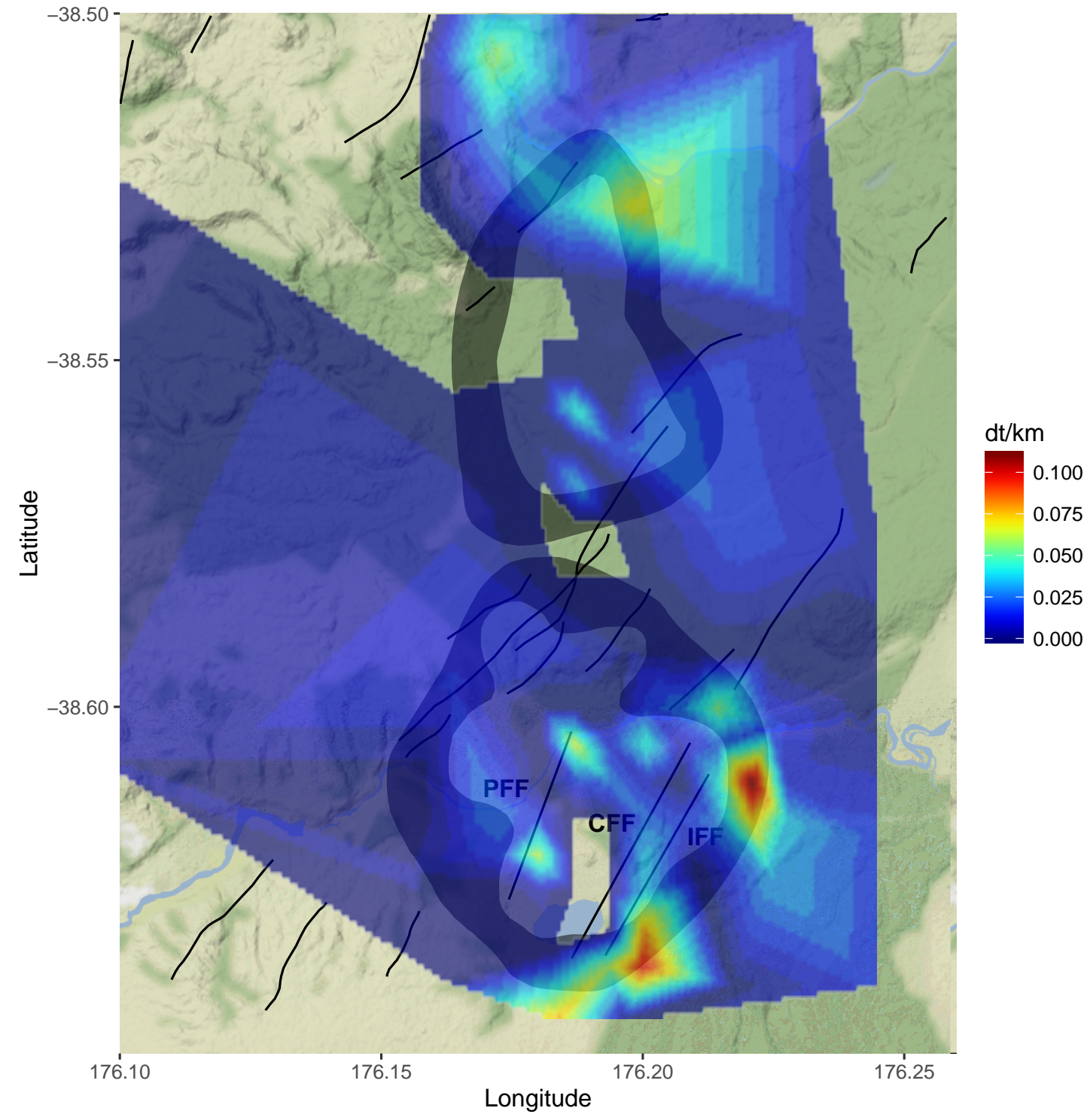

Figure 4.28: Delay time tomography for the full data set. Determined with the TESSA package and plotted with linear interpolation. Resistivity boundaries for the fields are outlined in grey. Black lines represent fault traces. 


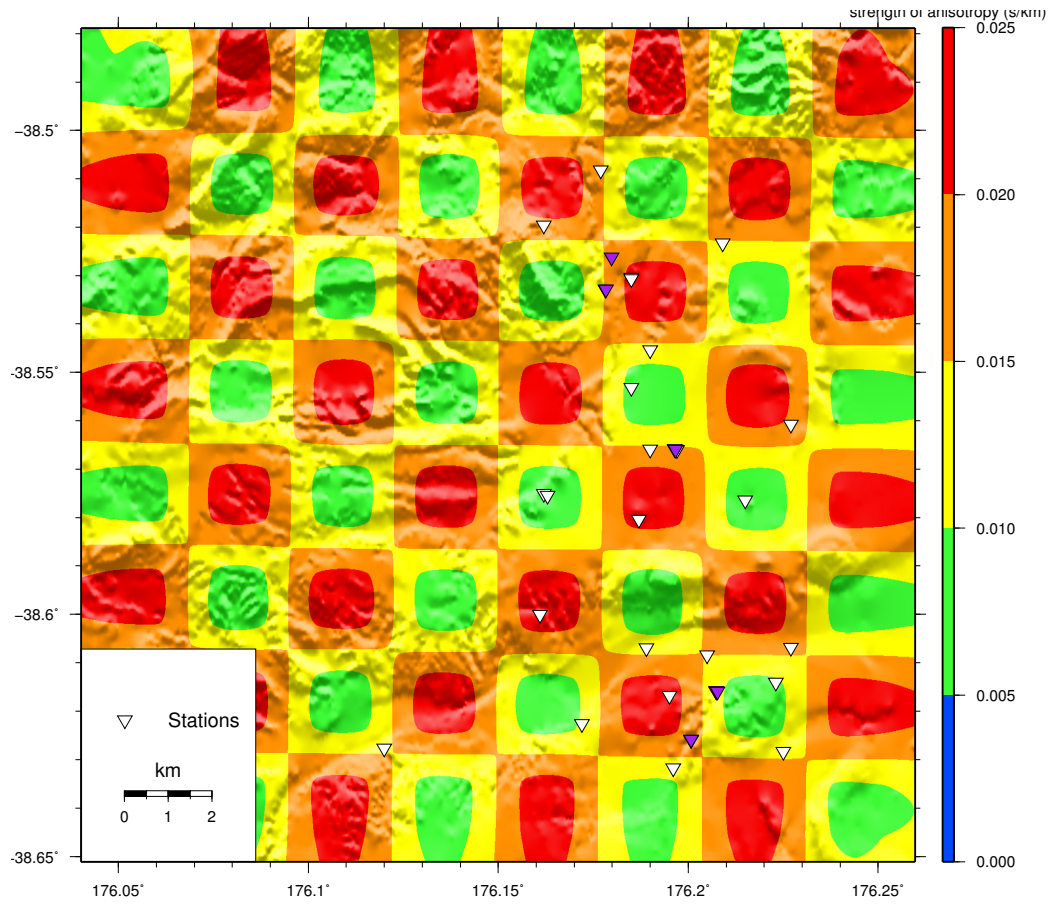

(a) Checkerboard test input model

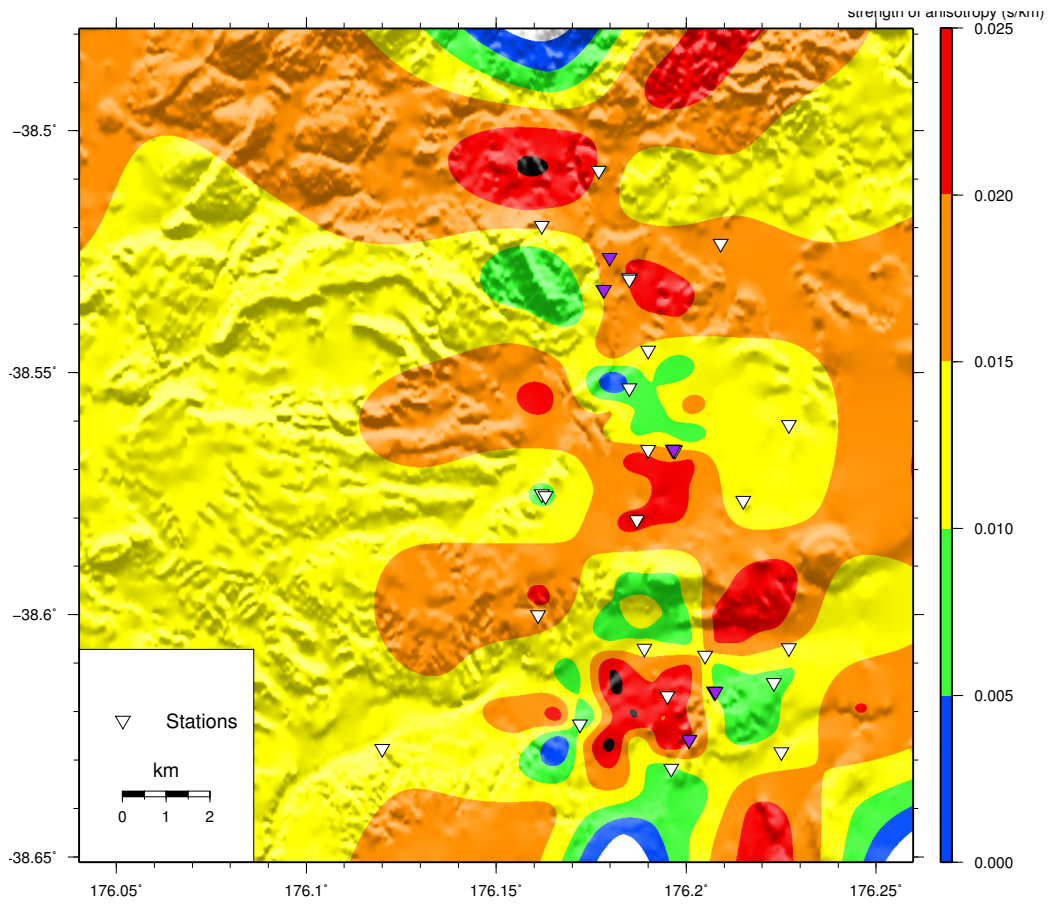

(b) Checkerboard test output

Figure 4.29: Checkerboard test for the full dataset. 


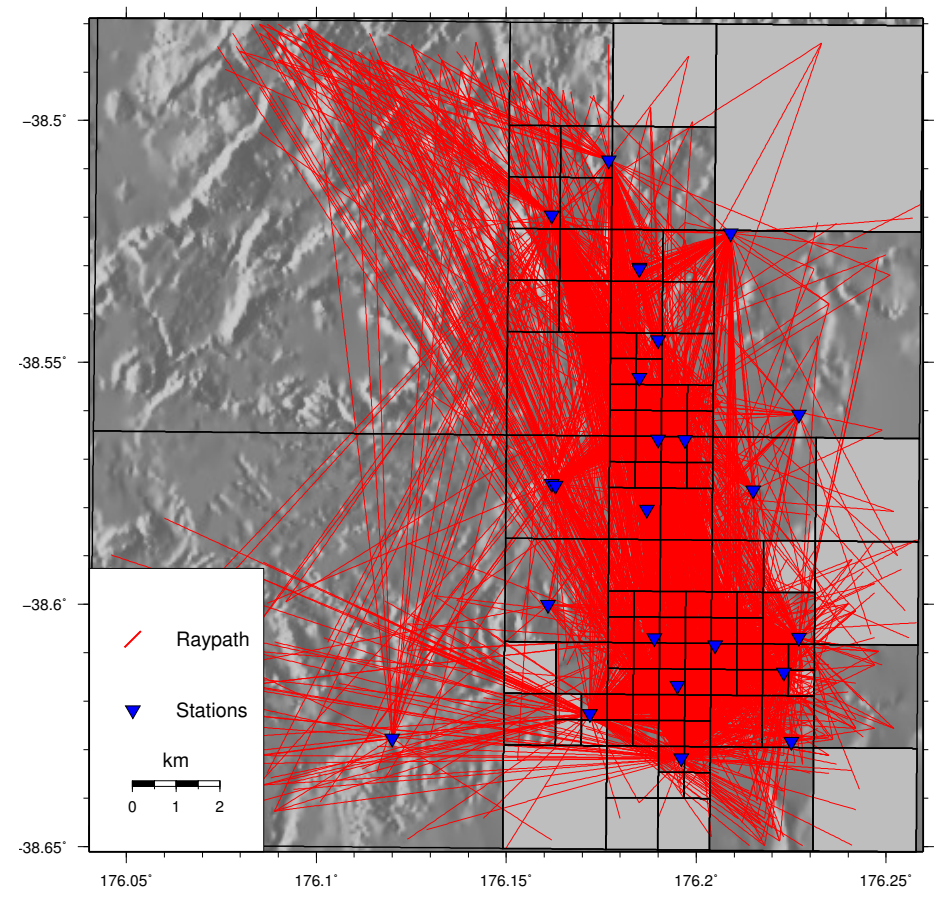

(a) Raypaths

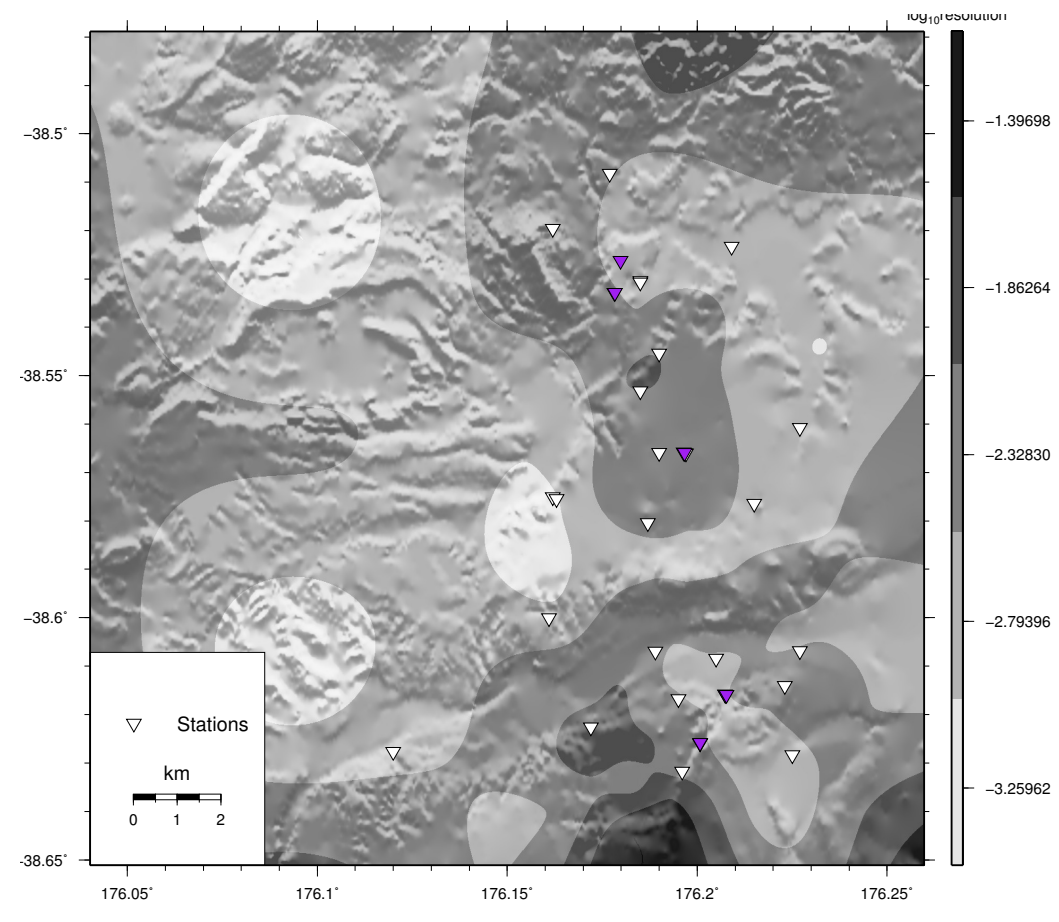

(b) Checkerboard test resolution

Figure 4.30: Raypaths and checkerboard test resolution for the full dataset. 
For spatial averaging, the dataset restriction's aim is to remove measurements travelling at shallow angles with respect to the symmetry axis of the anisotropy which may have their polarisation switched up to $90^{\circ}$.

The reduction in the number of measurements for the restricted dataset means that the number of grid boxes across the study area is greatly reduced. Average fast polarisations appear to all align with the expected NE-SW orientation in Ngatamariki. In Rotokawa, where resolution is better, polarisations are fairly uniformly closer to N-S.

As with the full dataset, we see a strong correlation with fault strikes especially in Rotokawa with polarisations aligning with the strike of the PFF, CFF and IFF.

Rotations of polarisations to NNE-SSW in eastern Rotokawa are no longer apparent.

\section{Delay time tomography}

Figure 4.32 shows the delay time tomography for the restricted dataset. Checkerboard test and residuals are shown in Figure 4.33 and 4.34 .

For the delay time tomography, the restriction should be removing measurements with artificially low time delays due to their propagation angle.

Again, delay time tomography suffers from the reduction of measurements in the restricted dataset. Inspection of the checkerboard test (Figure 4.33) shows that the high anisotropy anomaly in the south is an edge effect. Slightly higher than background anisotropy is imaged at Ngatamariki but with very low resolution in the area conclusions cannot be drawn.

The two high regions of anisotropy seen in the full dataset in south-eastern Rotokawa are no longer apparent. A region of high anisotropy appears centred on the production field fault. Regions of high anisotropy also appeared to be associated with the production field fault (PFF) for the full dataset. 


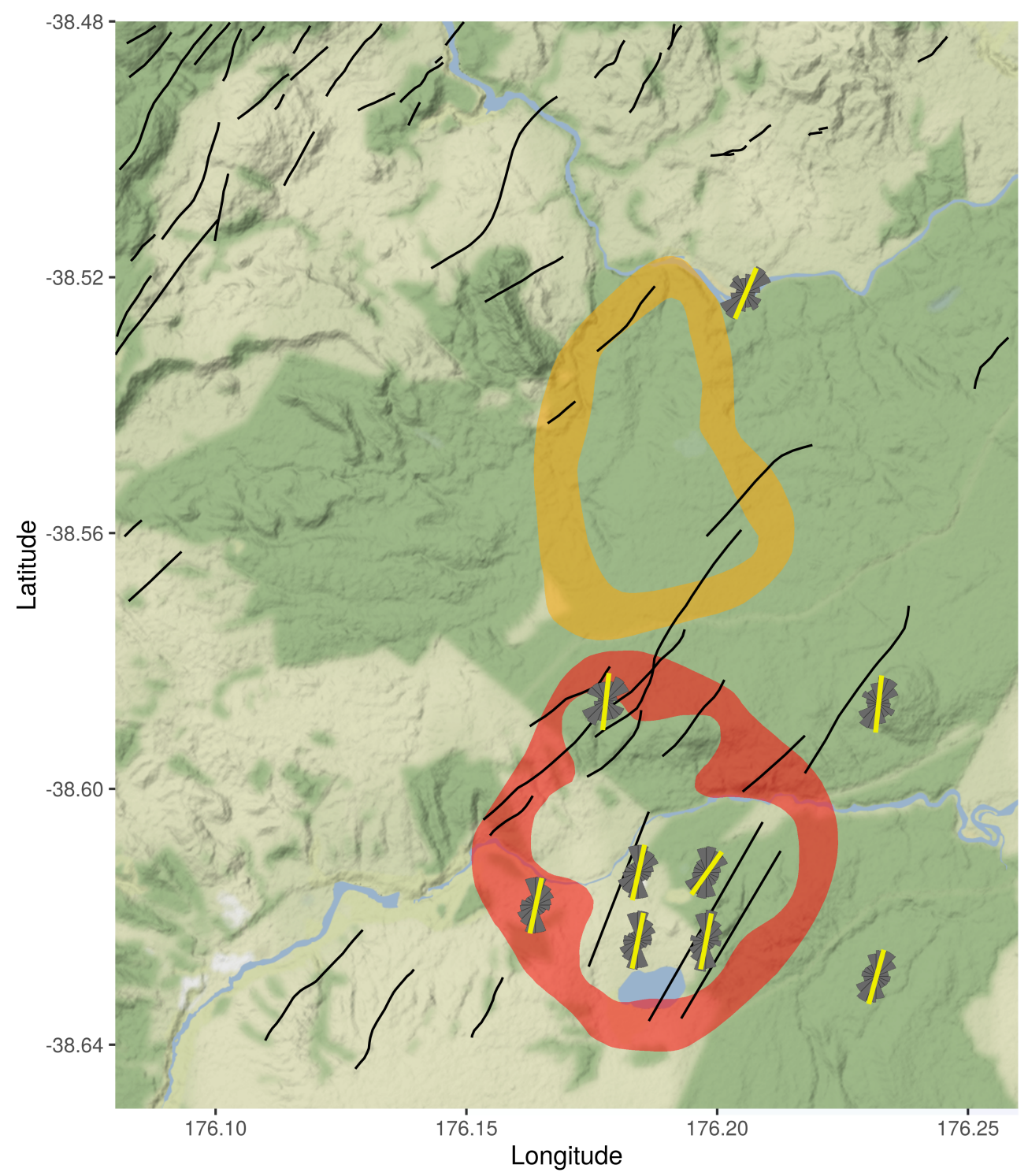

Figure 4.31: Spatial average of shear wave splitting fast polarisations ( $\phi$ ) for the restricted dataset. Determined with $1 / d^{2}$ weighting and quadtree gridding using the TESSA package. Rose plots with high standard error or standard deviation are not included. Resistivity boundaries for Rotokawa (south) and Ngatamariki (north). Black lines represent fault traces. 
The scale of $\delta t p k m$ has been changed for Figure 4.32, with a maximum corresponding to $0.08 \mathrm{~s} / \mathrm{km}$ rather than the $0.13 \mathrm{~s} / \mathrm{km}$ of Figure 4.28 . This is interesting as restricting the dataset should be removing measurements with anomalously low time delays. It is probable that the peaks we see in Figure 4.28 are artifacts of the tomography. This is supported by the checkerboard test of the full dataset (Figure 4.29) where higher anisotropy is returned than is present in the checkerboard. TESSA's strength lies in detecting regions of higher anisotropy rather than giving a necessarily accurate estimate of what the true $\delta t p k m$ is (Johnson et al. 2011). 


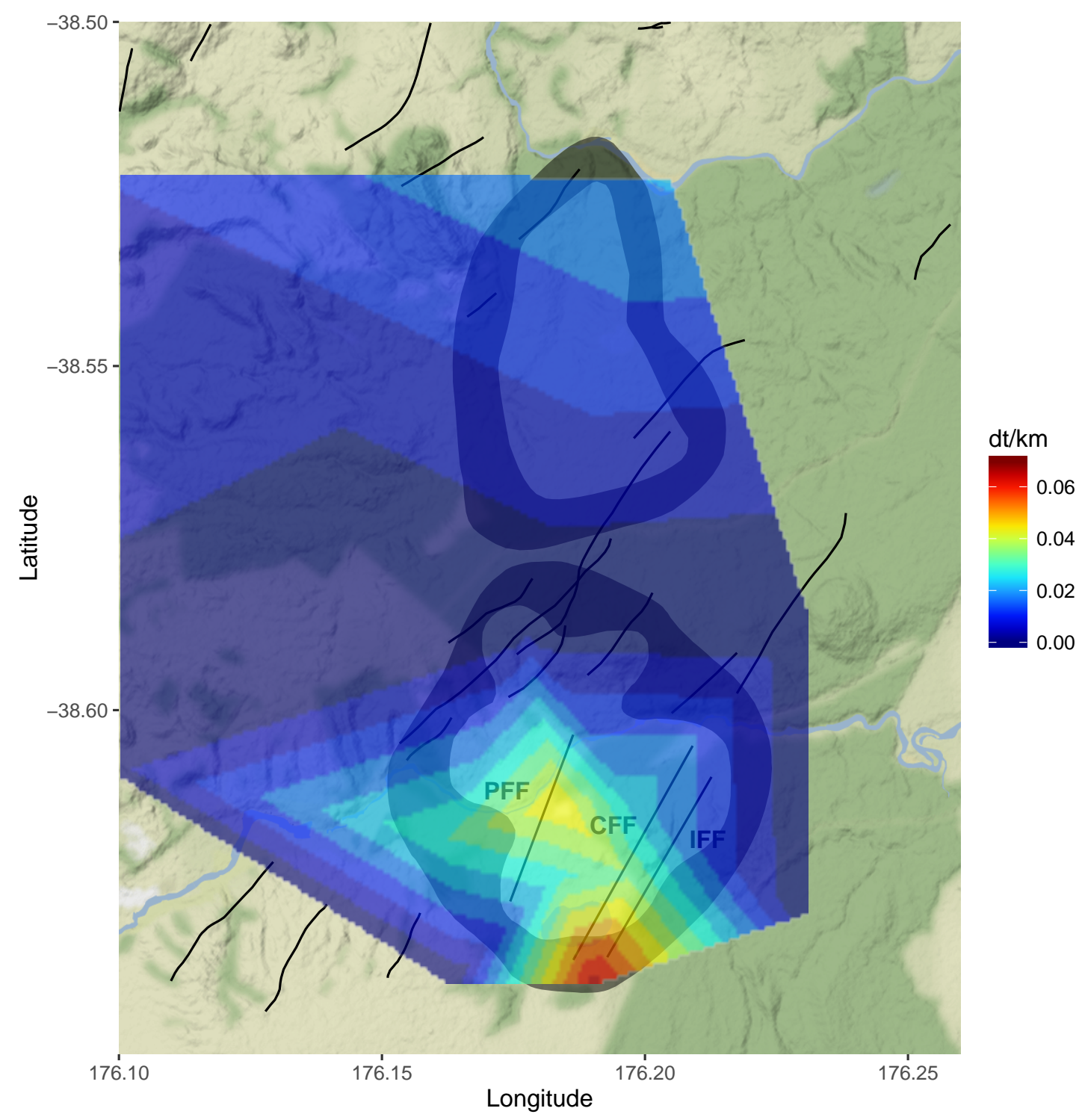

Figure 4.32: Delay time tomography for the restricted data set. Determined with the TESSA package and plotted with linear interpolation. Note the scale difference from Figure 4.28. Resistivity boundaries for the fields are outlined in grey. Black lines represent fault traces. 


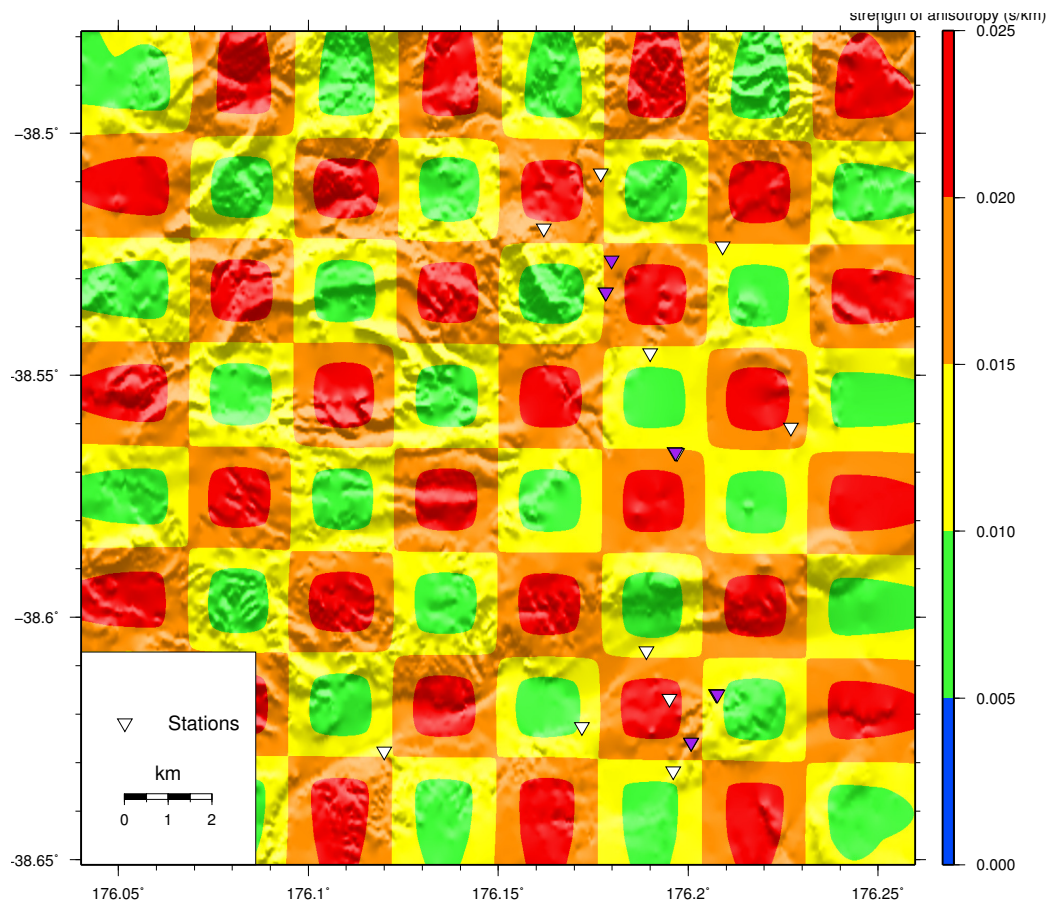

(a) Checkerboard test input model

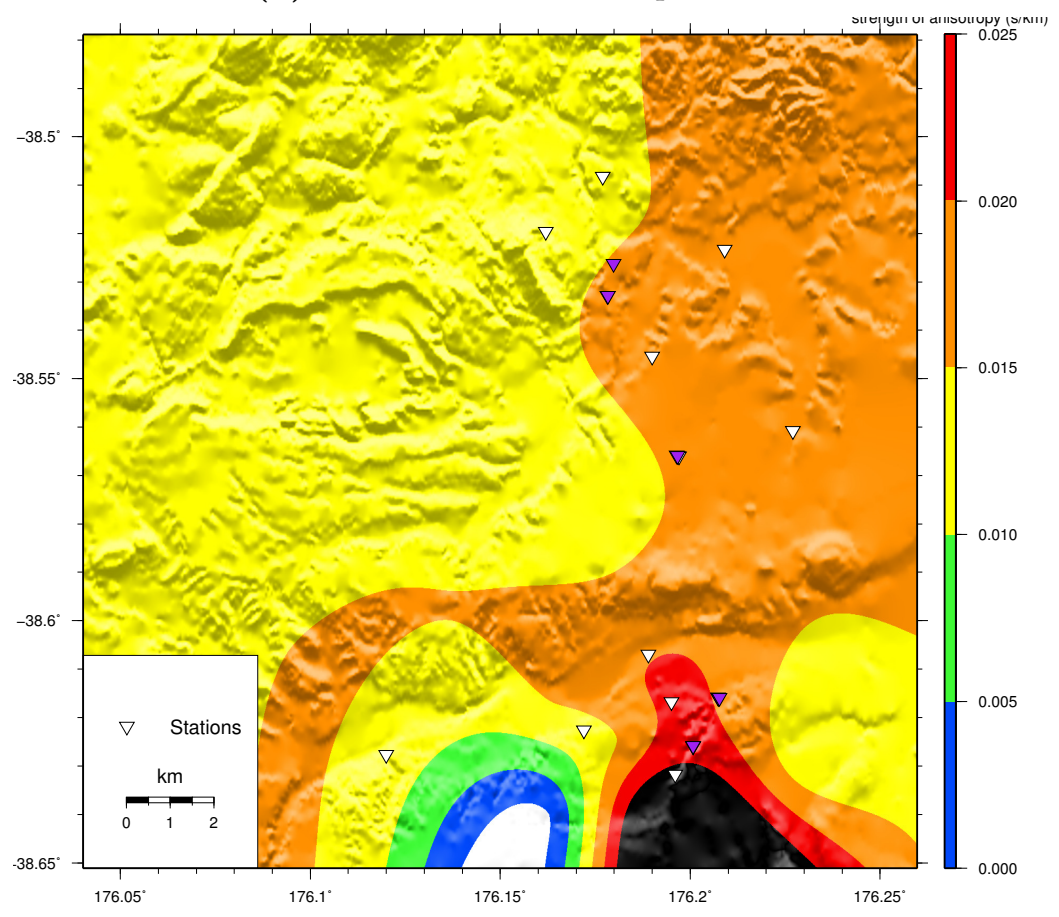

(b) Checkerboard test output

Figure 4.33: Raypaths and checkerboard test resolution for the restricted dataset. 


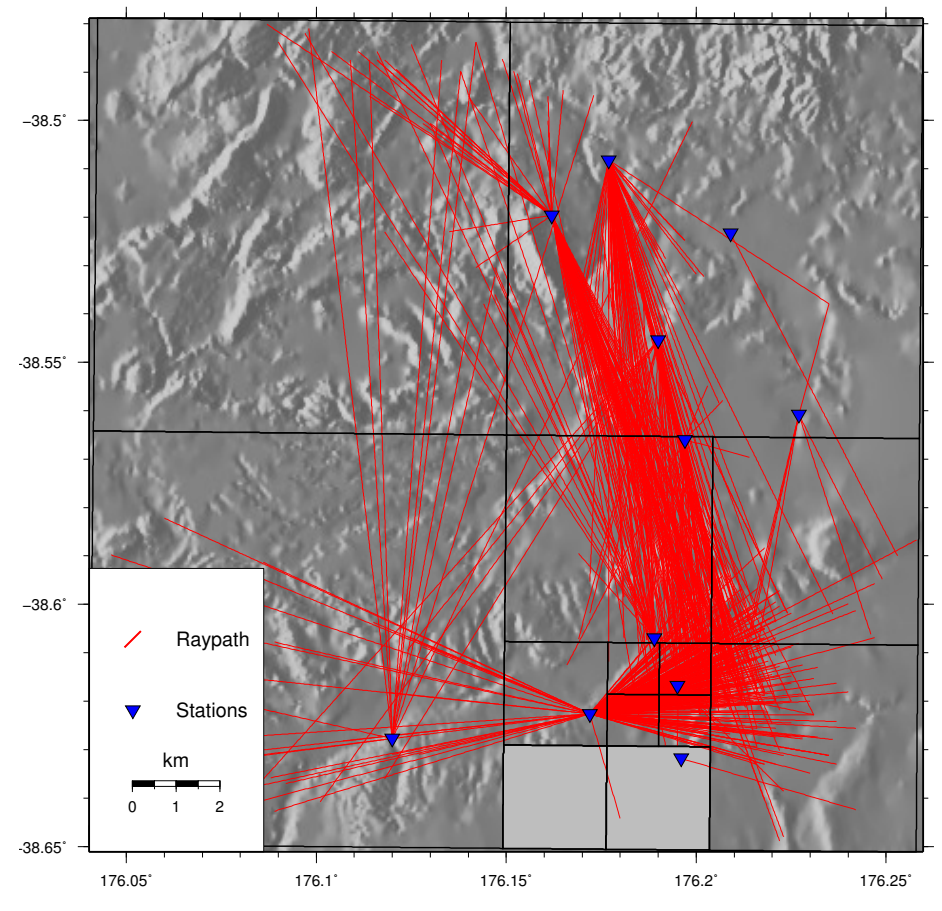

(a) Raypaths

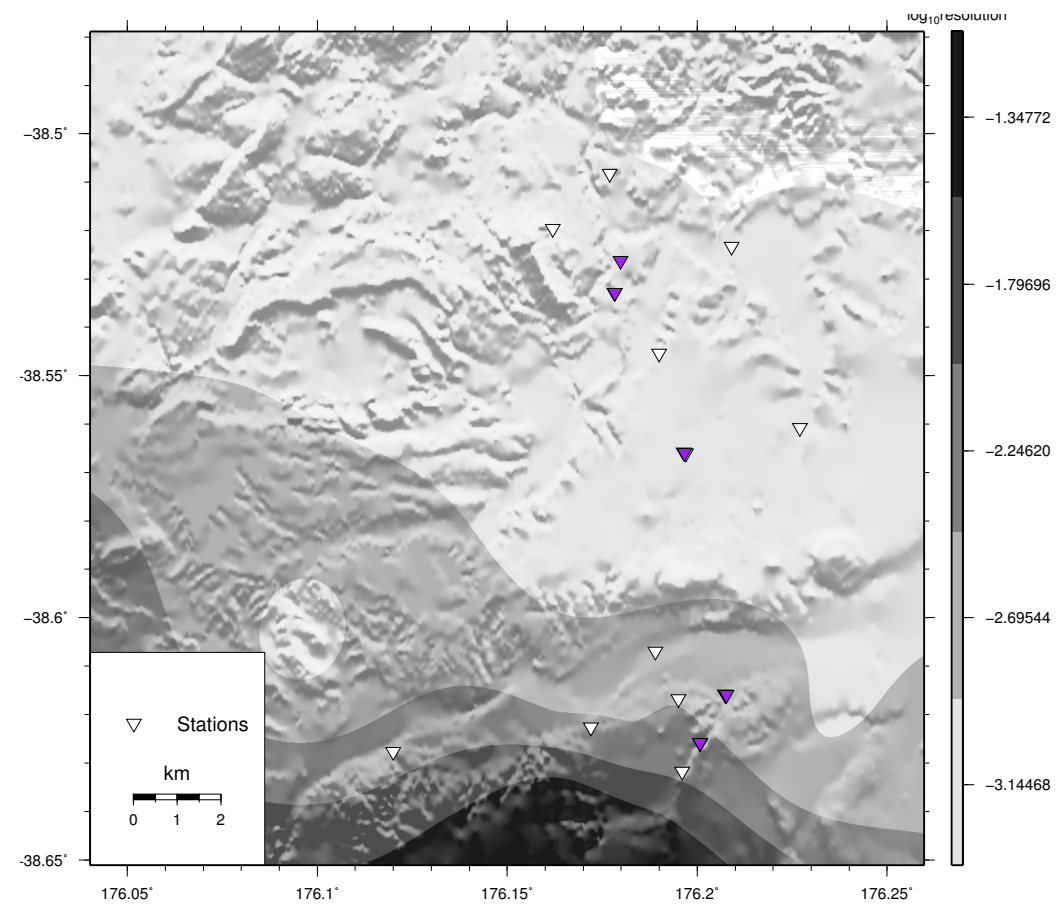

(b) Checkerboard test resolution

Figure 4.34: Checkerboard test and raypaths for the restricted dataset. 


\section{6 $v_{P} / v_{S}$ ratios}

Figure 4.35 illustrates the range of $v_{P} / v_{S}$ ratios, which form an almost a perfect normal distribution. This is likely an example of the Central Limit Theorem where a large number of random variables (not necessarily normally distributed themselves) that are controlling $v_{P} / v_{S}$ sum to a normal distribution. The mean $v_{P} / v_{S}$ for all events is 1.64 .

On inspection of Figure 4.35, we have $v_{P} / v_{S}$ ratios which vary from very low to high. As we are averaging over the entire path length, paths with low $v_{P} / v_{S}$ indicated that the majority of the cracks are probably gas filled while, on the other end of the scale, the majority of cracks are water filled. Combining this with varying crack densities, a normal distribution results.

\subsection{1 $v_{P} / v_{S}$ and delay time}

Correlation should be present between delay time, normalised by path distance $(\delta t p k m)$, and $v_{P} / v_{S}$ if anisotropy is originating from fluid-filled microcracks, with positive correlation for saturated cracks (Unglert et al. 2011). For dry (gas filled) cracks $v_{P}$ is lowered thus the ratio $v_{P} / v_{S}$ is also reduced. For saturated (water filled) cracks $v_{S}$ is reduced while $v_{P}$ remains largely constant thus ratio $v_{P} / v_{S}$ is increased. When we measure increased delay time, if the anisotropy is originating from fluid-filled cracks, this corresponds to increased crack density. So, the higher the crack density the more $v_{P} / v_{S}$ will be changed which results in a correlation between $v_{P} / v_{S}$ and $\delta t p k m$. Correlations and their significance are calculated using Pearson's moment correlation (Section 3.2.5).

We see a statistically significant positive correlation between $v_{P} / v_{S}$ and $\delta t p k m$. This indicates that the majority of cracks are saturated and not gas filled. This correlation also gives some indication that the source of the anisotropy is stress 


\section{Histogram of Vp/Vs with Normal Curve}

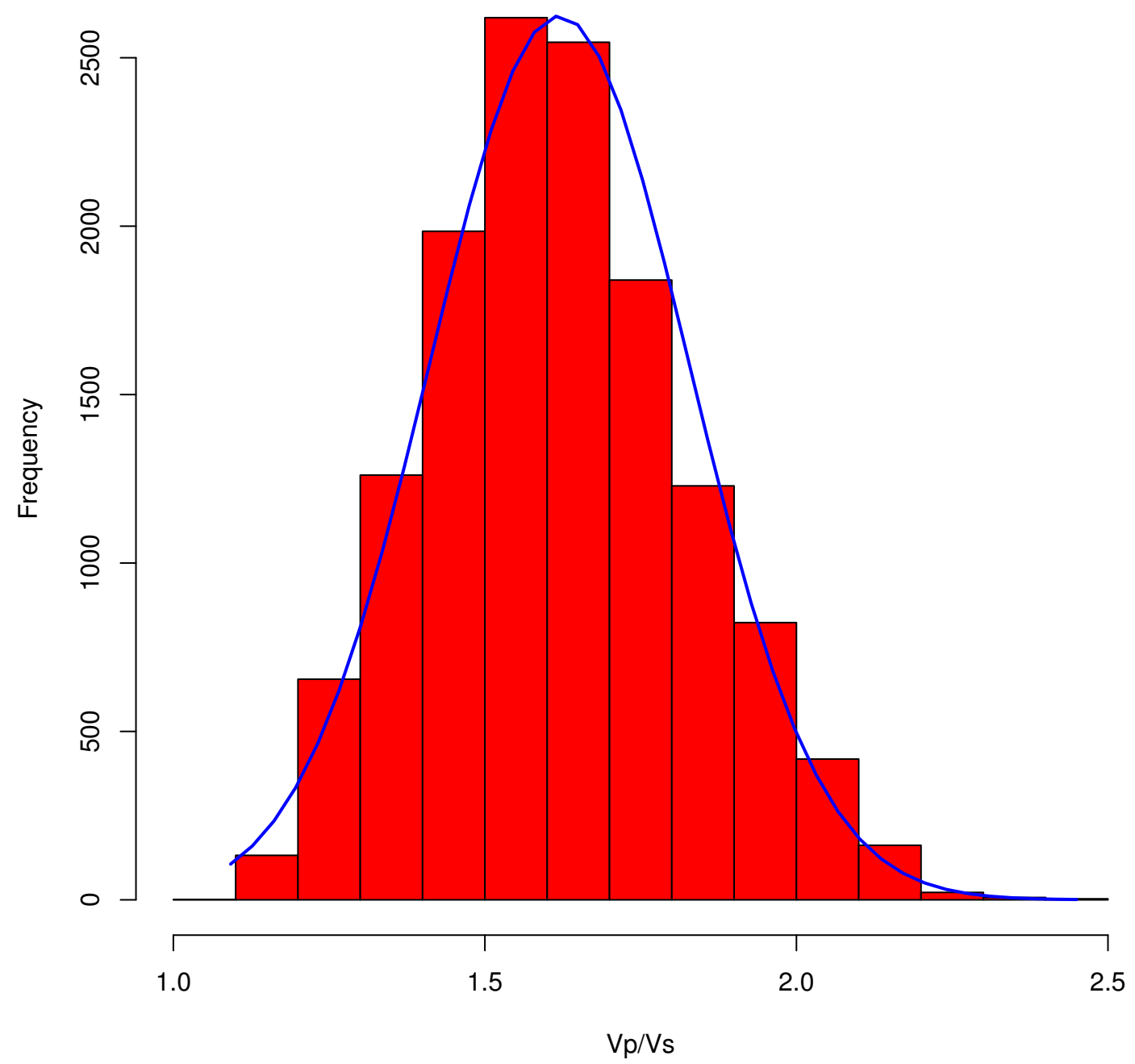

Figure 4.35: Histogram of $v_{P} / v_{S}$ ratios fit with a normal curve. Mean $v_{P} / v_{S}=$ 1.64 . 


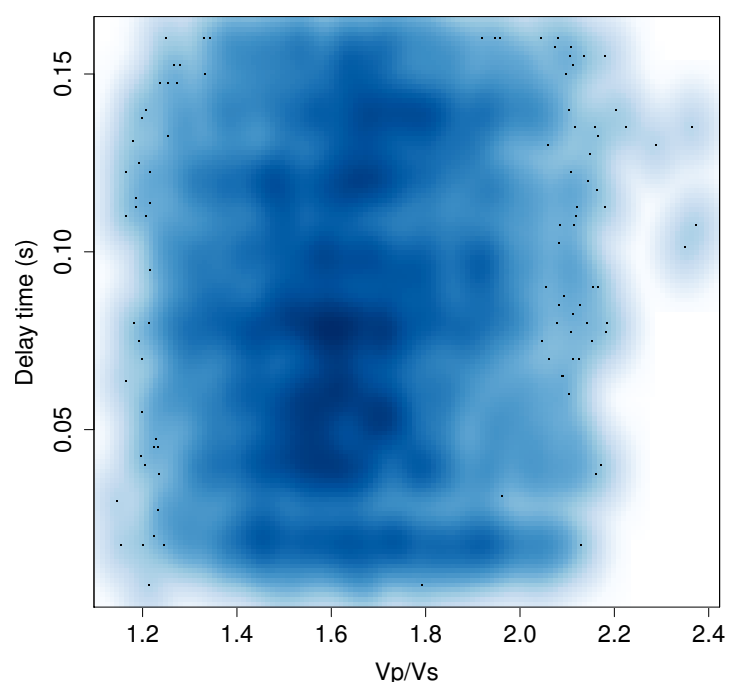

(a) $v_{P} / v_{S}$ versus delay time

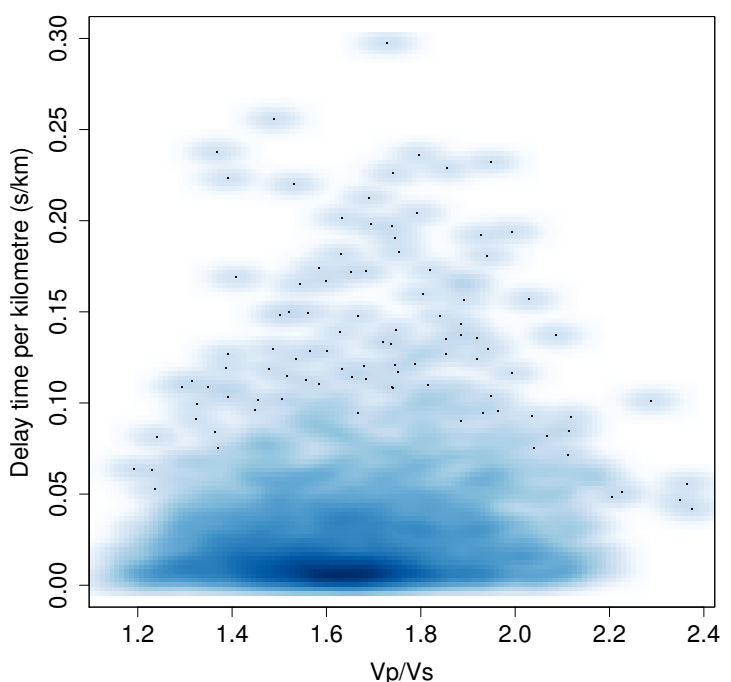

(b) $v_{P} / v_{S}$ vs delay time per kilometre

Figure 4.36: Smoothed color density scatter plot of delay time and $v_{P} / v_{S}$ for the full dataset (see Section 4.6.1). Darker regions signify a higher density of points.

aligned fluid-filled cracks (Unglert et al. 2011).

\section{Full dataset}

Correlation between $v_{P} / v_{S}$ and $\delta t p k m$ gives a small coefficient of 0.077 with a very low p-value of $1.53 * 10^{-5}$ indicating that the correlation is not zero. Plots are illustrated in Figure 4.36.

\section{Restricted dataset}

We use the results of MSAT crack fitting (Section 3.8) to restrict the data set to those measurements used in the tomography with TESSA (described in Section 3.9.1) which should have consistent delay times which do not vary with propagation angle (Figure 4.37). 
Saturated cracks work to reduce $v_{S}$ while $v_{P}$ remains largely unchanged thus $v_{P} / v_{S}$ increases. Increased delay time corresponds to an increase in crack density and so the more $v_{P} / v_{S}$ is increased. Thus, for saturated cracks, we expect a positive correlation between $v_{P} / v_{S}$ and $\delta t p k m$. For the restricted dataset we are removing measurements that will theoretically have, given their propagation angles, anomalously low delay times and so a correlation between $v_{P} / v_{S}$ and delay time, if present, should increase.

$v_{P} / v_{S}$ and $\delta t p k m$ shows a correlation coefficient, much higher than that for the full dataset, of 0.29. An increase in the correlation is what we would expect for the restricted dataset if our restriction is having the desired effect. The very large increase in correlation between $v_{P} / v_{S}$ and $\delta t p k m$, of 0.077 for the full data set to 0.29 for the restricted dataset, strongly suggests that this is the case.

\subsection{2 $v_{P} / v_{S}$ and depth}

Figure 4.38 illustrates how $v_{P} / v_{S}$ varies with depth of the earthquake. There is a strong negative correlation (-0.24) which translates to lower a $v_{P} / v_{S}$ for events occurring at greater depth. This can be seen visually on Figure 4.38 where below depths of $\sim 2.5 \mathrm{~km}$ the upper limit of $v_{P} / v_{S}$ drops off in a linear fashion.

This result is expected as low S-wave velocity $\left(v_{S}\right)$ layers in the near the surface increase $v_{P} / v_{S}$ in this region. Earthquakes occurring at shallower depths travel largely through these high $v_{P} / v_{S}$ layers while for deeper earthquakes the travel paths have a smaller percentage of their total path through them.

While further investigation into $v_{P} / v_{S}$ is out of the scope of this thesis, there is definitely variation and application of $v_{P} / v_{S}$ tomography would aid in revealing it. $v_{P} / v_{S}$ tomography would help to highlight regions of high fracturing and high fluid (including gas/steam) content and their associated structures, which is useful knowledge for understanding geothermal reservoirs (e.g. Muksin et al. 2013). 


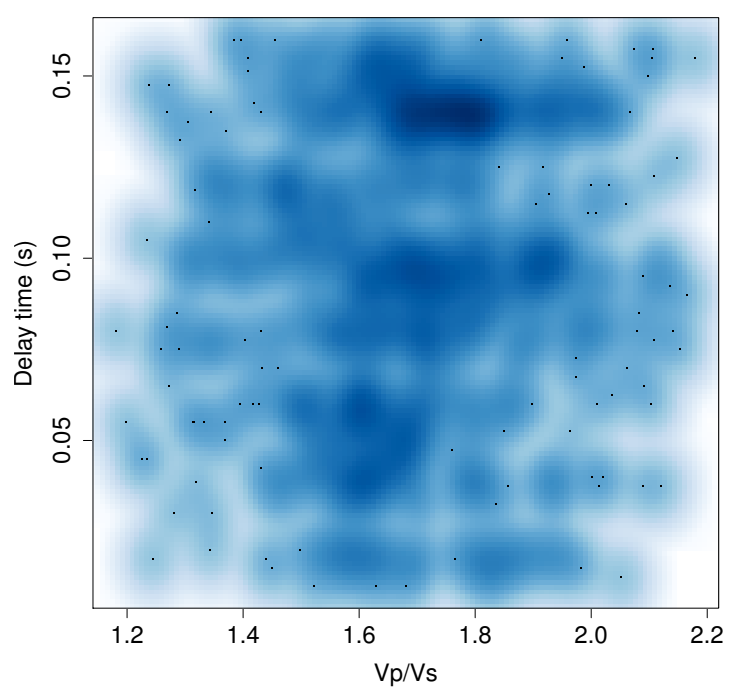

(a) $v_{P} / v_{S}$ versus delay time

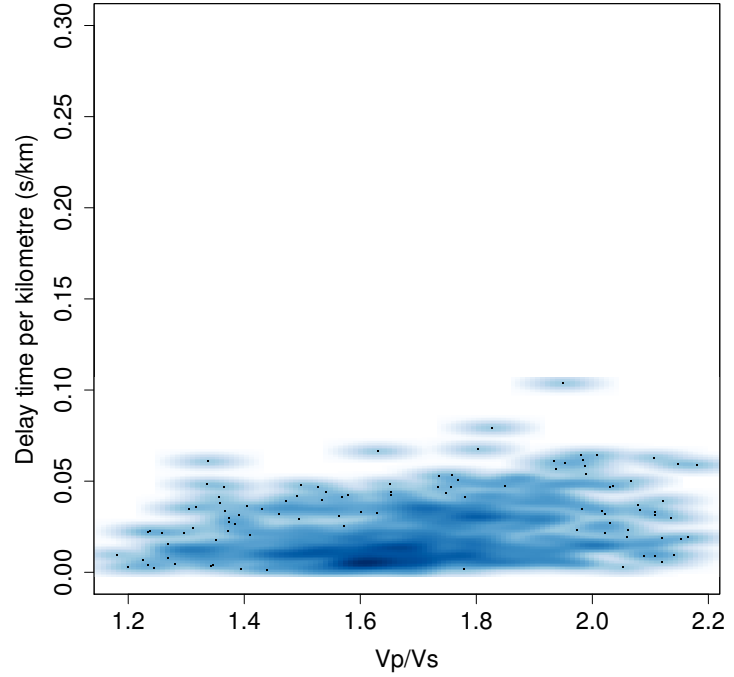

(b) $v_{P} / v_{S}$ vs delay time per kilometre

Figure 4.37: Smoothed color density scatter plot of delay time and $v_{P} / v_{S}$ for the restricted dataset (see Section 4.6.1). Darker regions signify a higher density of points. 


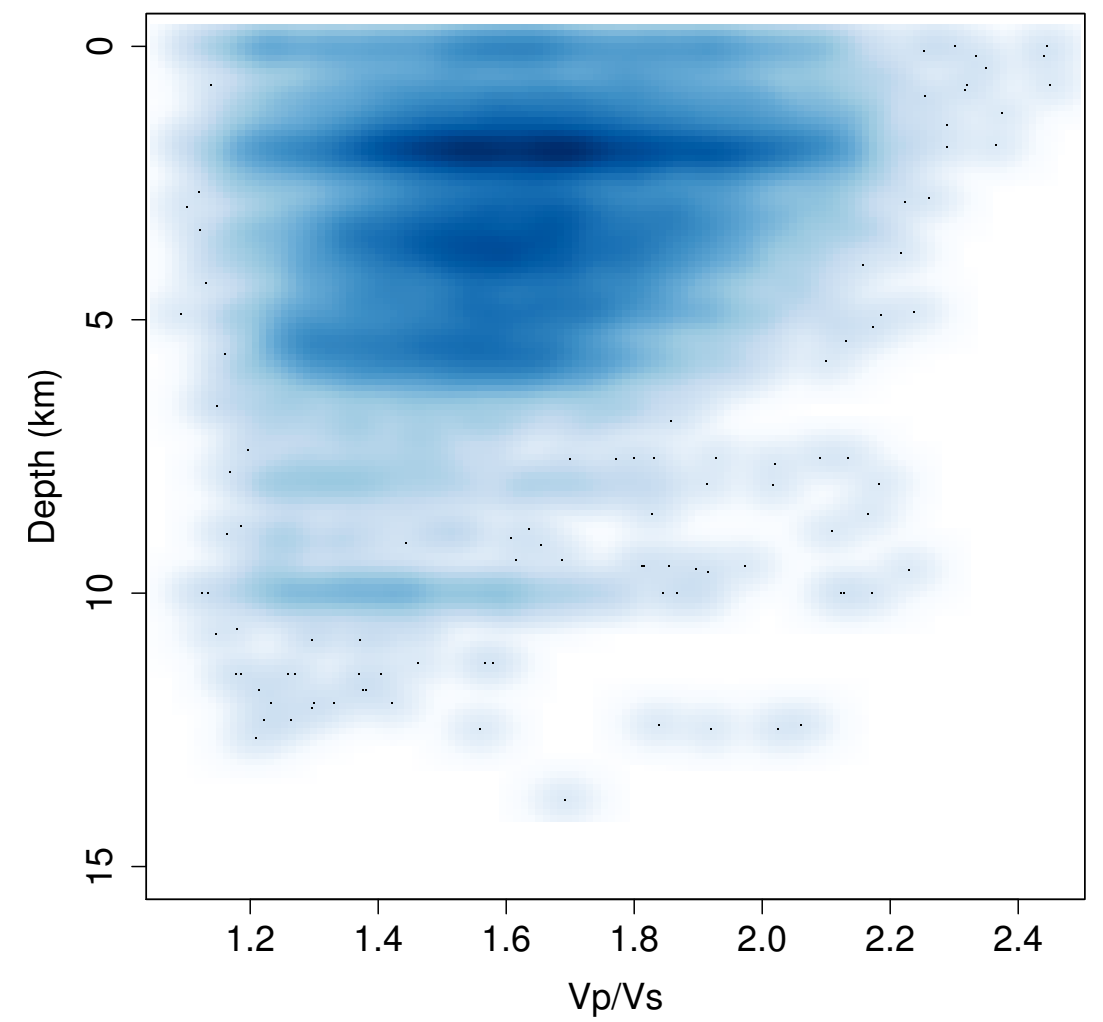

Figure 4.38: Smoothed color density scatter plot of $v_{P} / v_{S}$ versus depth. Darker regions signify a higher density of points. 


\section{Chapter 5}

\section{Further discussion}

\subsection{The shear wave window}

At angles of incidence greater than $35^{\circ}$ (where vertical incidence is $0^{\circ}$ ) converted phases can interfere with shear wave splitting measurements at the surface (Nuttli 1961; Booth and Crampin 1985). Events with angles of incidence below $35^{\circ}$ fall into the shear wave window where measurements are free of this interference. Low velocity layers cause a steepening of propagation angles so, for uniform velocity models or those that are missing low velocity layers (e.g. above sea level), angle of incidence at the surface can be overestimated. So an event with a theoretical incidence angle greater than $35^{\circ}$ is likely still within the shear wave window. Due to these low velocity layers near the surface, many studies consider events with angles of incidence below $45^{\circ}$ (for a uniform velocity model) to be in the shear wave window (e.g. Volti and Crampin 2003). However, this is too restrictive and so removes many useful measurements (Savage et al. 2010b).

Angle of incidence being overestimated by a uniform velocity model is especially the case in geothermal areas, which are characterised by low velocities. For example, $64 \%$ of measurements with angles of incidence greater than $45^{\circ}$ on Figure 5.1 
(determined from a uniform velocity model, Section 3.7) are in fact within the shear wave window when angles of incidence are calculated using a more accurate velocity model (Section 3.6.2). These angles of incidence are calculated at sea level and most stations sit at roughly $400 \mathrm{~m}$ above sea level. Thus, even removing measurements with angles of incidence greater than $35^{\circ}$ (with the improved velocity model) would be too restrictive. The top layer in our velocity model estimates an S-wave velocity of $1.14 \mathrm{~km} / \mathrm{s}$ however, imaging of the top $200 \mathrm{~m}$ in Ngatamariki conducted by Civilini et al. (2016) with an ambient noise method, reveals velocities below $0.4 \mathrm{~km} / \mathrm{s}$.

Low velocity layers above sea level mean that the vast majority of our shear wave splitting measurements are within the shear wave window. Our crack fitting process is also improved by measurements at shallow propagation angles (and thus shallow angles of incidence) and so not removing them is beneficial. As such, we choose not to remove any measurements based on their angles of incidence with the surface. 


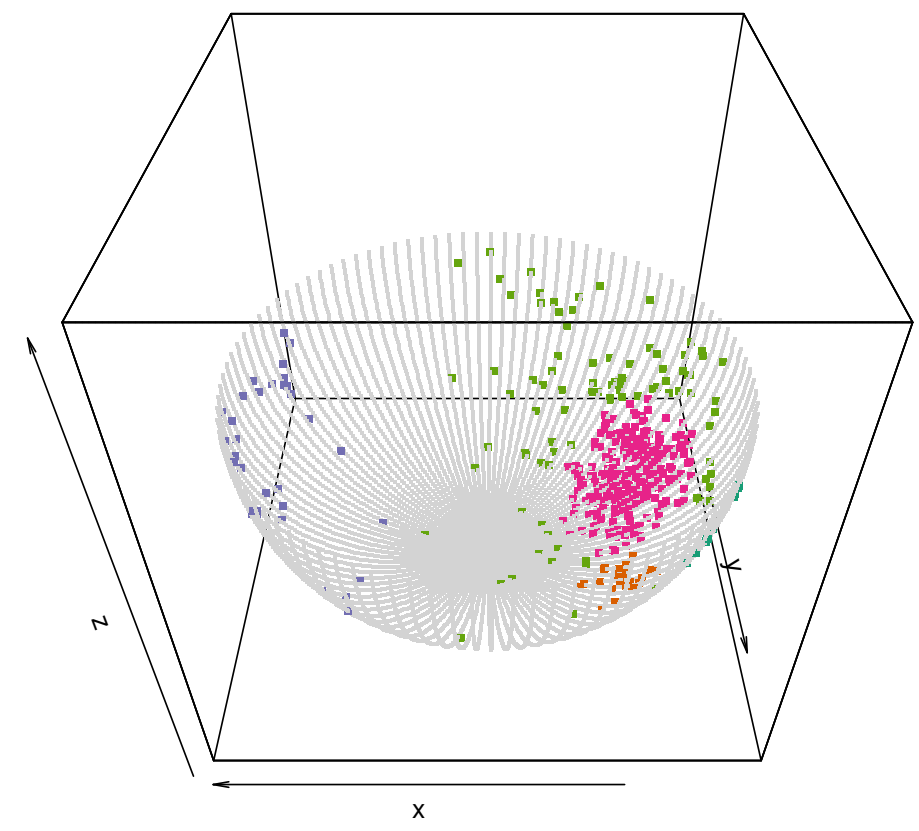

Figure 5.1: Clustered station-event pierce points for station RT01 shown on a unit hemisphere below the station. Each pint corresponds to a back azimuth and angle of incidence determined from a uniform velocity model. 


\subsection{Borehole versus surface stations}

Orientations for the three Ngatamariki stations (NS12, NS13, \& NS13) are determined by the Rayleigh wave method (Section 3.3) and are quoted in Table 5.1 along with their elevations.

The elevations of our borehole stations are much closer to sea level and so the angles of incidence we calculate (Section 3.6.2) will be closer to the actual angles of incidence than for surface stations. Thus, measurements which we may have correctly assumed were within the shear wave window (Section 5.1) for a surface station may not be for these borehole stations.

Roughly $30 \%$ of measurements for each station have estimated angles of incidence greater than $35^{\circ}$. We apply the Rayleigh test (Section 3.2.3) to test for a dominant mean for polarisations from the two sets of measurements with angles of incidence $>35^{\circ}$ and $\leq 35^{\circ}$ for each station. Of the three borehole stations, only NS13 passes the Rayleigh test (with a p-value < 0.05) for both sets. Applying Watson's test (Section 3.2.4) to the two sets for measurements with angles of incidence $>35^{\circ}$ and those $\leq 35^{\circ}$ (returning a p-value of 0.5 ). The same was found for a stricter cut off of $30^{\circ}$.

Since both NS12 and NS14 do not appear to have a dominant mean orientation we cannot determine if the shear wave window could be affecting the data quality. However, NS13 shows no difference in mean orientation for polarisations for measurements above and below a $35^{\circ}$ angle of incidence so it appears that any measurements outside the shear wave window are a small minority even for angles of incidence estimated to be above $35^{\circ}$. We extend this to NS12 and NS14 and assume that a negligible amount of measurements are outside the shear wave window.

NS12 and NS14, which both fail the Rayleigh test for their shear wave splitting fast polarisations, have their orientations determined to a lesser accuracy than NS13 (Section 4.1). As the station orientations are determined by measuring Rayleigh 
Table 5.1: Borehole stations with orientation (of the North component) and elevation (metres above sea level).

\begin{tabular}{|c|c|c|}
\hline Station & Orientation $\left(^{\circ}\right)$ & Elevation $(\mathrm{m})$ \\
\hline \hline NS12 & 285.73 & -164 \\
\hline NS13 & 292.03 & 130 \\
\hline NS14 & 65.31 & 163 \\
\hline
\end{tabular}

wave polarisations it is possible that a similar mechanism to what is causing the large scatter was see in shear wave splitting polarisations is also affecting our orientations.

\subsection{Grades}

We apply separate grades of F1, F2 and F3 to our shear wave splitting measurements following the method of Castellazzi et al. (2015) (Section 3.6.1). We use this grading technique to remove measurements which are inconsistent between different filters which otherwise pass $\mathrm{AB}+$ grade. Castellazzi et al. (2015) goes on to treat the grades separately however, we do not. Removing all F1 measurements would reduce our number of high grade measurements by more than half. Table 5.2 shows the counts of each grade per station as well the p-values from applying Watson's test (Section 3.2.4) to the fast polarisations to compare the means of the three grades. Of the 17 stations with enough measurements to run Watson's test, only three had any evidence $(\mathrm{p}$-value $\leq 0.05)$ that the mean fast polarisation from the three grades differed.

We do not see much evidence of mean fast polarisations changing with grade. As we do not want to remove a significant number of measurements which are likely accurate, we choose to treat all high grade measurements (F1, F2, \& F3) equally.

A potential future approach would be to weight the grades separately. For ex- 
ample, when treating polarisations as point masses for the calculation of their mean (described in Section 3.2.1), the "mass" of each point can be reduced for F1 and F2 measurements. 
Table 5.2: Number of that particular grade for each station with p-values for Watson's test. NA indicates not enough measurements were available to run the test. 'Yes' indicates that there is no strong evidence that means from the three grades are different ( $p$-value $>0.05)$.

\begin{tabular}{|c|c|c|c|c|c|}
\hline Station & F1 & F2 & F3 & p-value & $>0.05 ?$ \\
\hline \hline ARAZ & 19 & 3 & 1 & NA & NA \\
\hline NS01 & 98 & 30 & 19 & 0.648 & Yes \\
\hline NS03 & 68 & 36 & 30 & 0.024 & No \\
\hline NS07 & 9 & 14 & 6 & 0.787 & Yes \\
\hline NS18 & 12 & 6 & 7 & 0.1 & Yes \\
\hline NS16 & 65 & 62 & 16 & 0.363 & Yes \\
\hline NS11 & 27 & 3 & 1 & NA & NA \\
\hline NS12 & 40 & 10 & 8 & 0.265 & Yes \\
\hline NS13 & 42 & 25 & 30 & 0.028 & No \\
\hline NS14 & 73 & 20 & 15 & 0.144 & Yes \\
\hline NS15 & 27 & 8 & 5 & 0.273 & Yes \\
\hline RT01 & 258 & 104 & 91 & 0.002 & No \\
\hline RT05 & 295 & 134 & 141 & 0.823 & Yes \\
\hline RT12 & 137 & 66 & 25 & 0.684 & Yes \\
\hline RT14 & 27 & 16 & 11 & 0.932 & Yes \\
\hline RT17 & 120 & 60 & 46 & 0.963 & Yes \\
\hline RT18 & 133 & 102 & 28 & 0.411 & Yes \\
\hline RT19 & 9 & 6 & 0 & NA & NA \\
\hline RT21 & 16 & 2 & 1 & NA & NA \\
\hline RT22 & 17 & 1 & 0 & NA & NA \\
\hline RT23 & 11 & 4 & 3 & NA & NA \\
\hline WPRZ & 136 & 65 & 44 & 0.647 & Yes \\
\hline
\end{tabular}




\subsection{Variations through time}

Having only eleven months of data, we do not have enough resolution to draw any conclusions about changes through time. There are no notable changes in reservoir stimulation during our study period. To test if there is any coarse change with time (which we do not expect), we divide polarisations into two groups from the first and second half of the study period respectively. We then apply Watson's test to compare their means. We find that all the stations, except NS16, have no strong evidence for any difference in mean fast polarisation (p-value 0.05). NS16 changed location during the study period which may explain it's change in mean polarisation.

\subsection{Measurement rates}

Table 5.3 summarises the differences in picking and measurement rate between the matched filter events and events from the GNS catalogue (averaged across each station from Table A.4). The biggest difference comes for picking the events with $17 \%$ for the catalogue events and only $10 \%$ for the matched filter events. Once a S-wave pick has been made the rate of high grade shear wave splitting measurements is very similar (34\% versus $32 \%)$. If the event is high enough quality for spickerC to make the S-wave pick then both catalogue and matched filter events are equally likely to have a high grade shear wave splitting measurement made. Despite the slightly lower picking and splitting rates, the addition of matched filter detections has greatly increased the number of high grade shear wave splitting measurements we have made from 1629 to 2944.

Our picking and splitting rates are significantly lower than that of Castellazzi et al. (2015) (for automatic shear wave splitting and picking on Mt Ruapehu with the same software) with $28 \%$ for S-wave picking (versus our 13\%) and $60 \%$ for shear wave splitting (versus our 32\%). This likely results from a difference in quality of 
Table 5.3: Mean percentage of measurements calculated from Table A.4. \% pick are the percentages of events which were successfully picked and \% sws are the percentage of the events with S-wave picked which resulted in high grade shear wave splitting measurements.

\begin{tabular}{|c|c|c|c|c|c|c|}
\hline \% det & \% norm pick & \% det pick & \% pick & \% norm sws & \% det sws & \% sws \\
\hline \hline 53 & 17 & 10 & 13 & 34 & 32 & 33 \\
\hline
\end{tabular}

the events. Castellazzi et al. (2015) only take events with magnitude 1.5 or greater. We do not filter by magnitude and, especially with the addition of low magnitude matched filter events, most of our earthquakes are even below magnitude 1.5. In addition, our study area is much smaller with significant anthropogenic noise originating from the power stations present in both fields.

\subsection{Theoretical distribution of polarisations}

Most shear wave splitting studies assume vertical cracks with sub-vertical propagation angles. This is not necessarily the case and in this thesis we have approached this problem by fitting theoretical crack planes to our data to allow the propagation angle, with respect to the axis of symmetry, to vary through its full range. However, propagation angle is often not well known. In this section we asses how well the von Mises distribution (Section 3.2.2) fits the underlying distribution of fast polarisations and how we can improve statistical tests and measures (especially mean and error) of crack orientation without knowledge of propagation angle.

We do not fit the von Mises distribution to our shear wave splitting fast polarisations. The underlying distribution of fast polarisations, if it were possible to sample randomly for real measurements, is not similar to the von Mises. To illustrate this we produce 2500 fast polarisations at randomly sampled values of propagation angle for 
a crack plane striking $045^{\circ}$ (from Figure 5.2a). Figure 5.2b shows the rose diagram of these sampled polarisations.

To compare this distribution to a von Mises, we fit a von Mises distribution to the (doubled) sampled polarisations using expectation maximisation (following the methods of Pewsey et al. (2013) and Agostinelli and Lund (2013)) and then assess the fit with Probability-Probability (P-P) and Quantile-Quantile (Q-Q) plots.

P-P and Q-Q plots provide an indication of the goodness of fit without testing it formally (Pewsey et al. 2013). P-P plots show the value of the probability density function for the von Mises distribution versus the empirical probability distribution function for the data being fit. For an ideal fit the values of both are equal. The same is true for the Q-Q plot however with the Quantile function instead.

Figure 5.2c shows the Q-Q and P-P plots. The further away the points lie from the dashed black line the worse the fit. While we do not asses the significance of these deviations it can be seen that the von Mises distribution does not appear to be a good fit. On Figures 5.2c and 5.3c, the points appear as a sold line due to their high number.

If we increase the dip of the crack plane to $060^{\circ}$, the (axial) bimodal nature becomes weaker (Figure 5.3). Because the unimodal character of the (doubled) fast polarisations is reduced, the power of the Rayleigh test (Section 3.2.3), to detect departures from uniformity, is reduced. As the dip of the cracks approaches $90^{\circ}$ (horizontal), the distribution of sampled fast polarisations approaches uniform. The increased level of fit we see in Figure 5.3c is due to the distribution being closer to a uniform distribution (the uniform distribution the same as a von Mises distribution with zero concentration).

It is apparent that, for a single hexagonal anisotropic layer, the underlying distribution of polarisations does not follow the von Mises distribution. In reality (where propagation angles are not sampled randomly, the single layer is an approximation, 
propagation angles aren't constant, and most measurements are sub-vertical) the distribution measured may still be approximated well by the von Mises distribution.

\subsubsection{Accounting for bimodality}

It is apparent that we are dealing with a bimodal axial distribution of underlying fast polarisations. These two modes, for randomly sampled propagation angles, fall $90^{\circ}$ from each other. Somewhat conveniently, cycle skipped polarisations also appear $90^{\circ}$ to strike of the cracks. Thus, just as the axial nature of our data introduced an $180^{\circ}$ ambiguity, this introduces a $90^{\circ}$ ambiguity. To collapse our points into a single direction, to allow us to make accurate estimates or tests, we can multiple our raw fast polarisations by four, apply the test or method, and then divide the answer by four. This is effectively multiplying our data by two twice, the first time to remove the $180^{\circ}$ ambiguity introduced for axial data, and the second time to remove the $180^{\circ}$ ambiguity present in the doubled polarisations due to our bimodal distribution.

The reason why this ambiguity can be generally overlooked is that, for random sampling and real applications where propagation angles are sub-vertical, most polarisations group around the strike of the cracks (e.g. as can be seen in Figure 5.4b). Thus, the mean fast polarisation will be close to the strike of the cracks.

By multiplying the fast polarisations by four, for our dataset of randomly sampled polarisations, the distribution is now truly unimodal. The strength of Rayleigh's test is increased and the fit of a von Mises distribution is much stronger (Figure 5.4) than that for the same distribution but only fitting to doubled polarisations (Figure 5.2). However, it is still apparent that it is far from ideal.

The Jones-Pewsey family of unimodal distributions includes the von Mises distribution with the addition of a shape parameter (Pewsey et al. 2013). A Jones-Pewsey distribution with a shape parameter equal to zero is equivalent to a von Mises dis- 

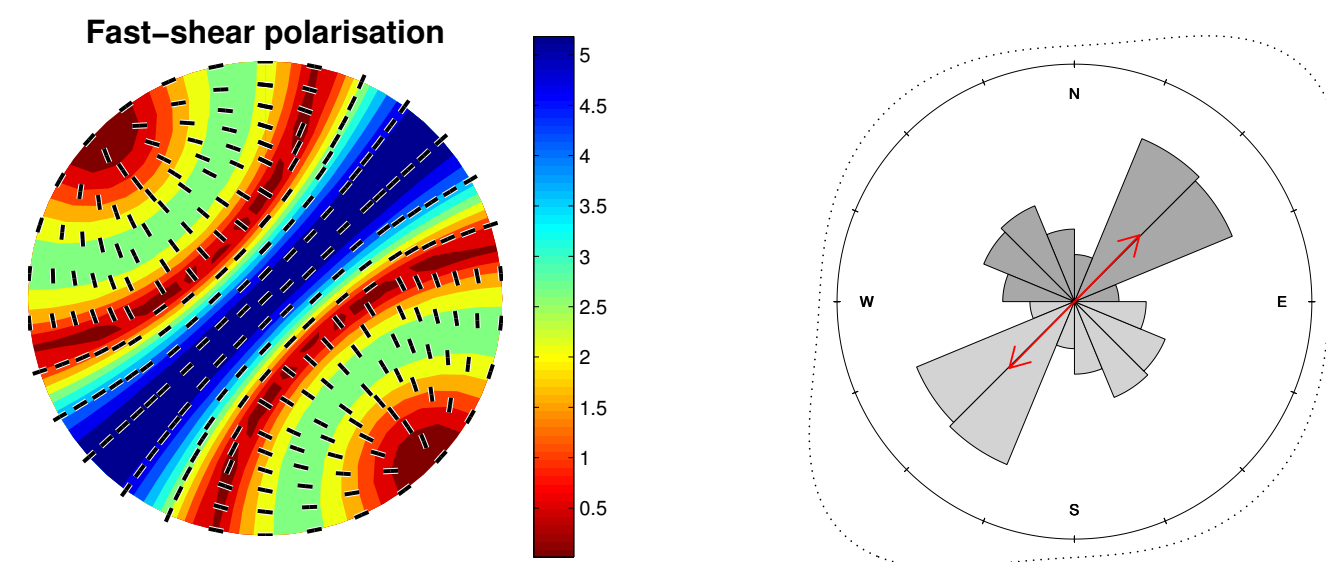

(a) Population of fast polarisations (black

(b) Rose diagram of sampled fast polarisalines)
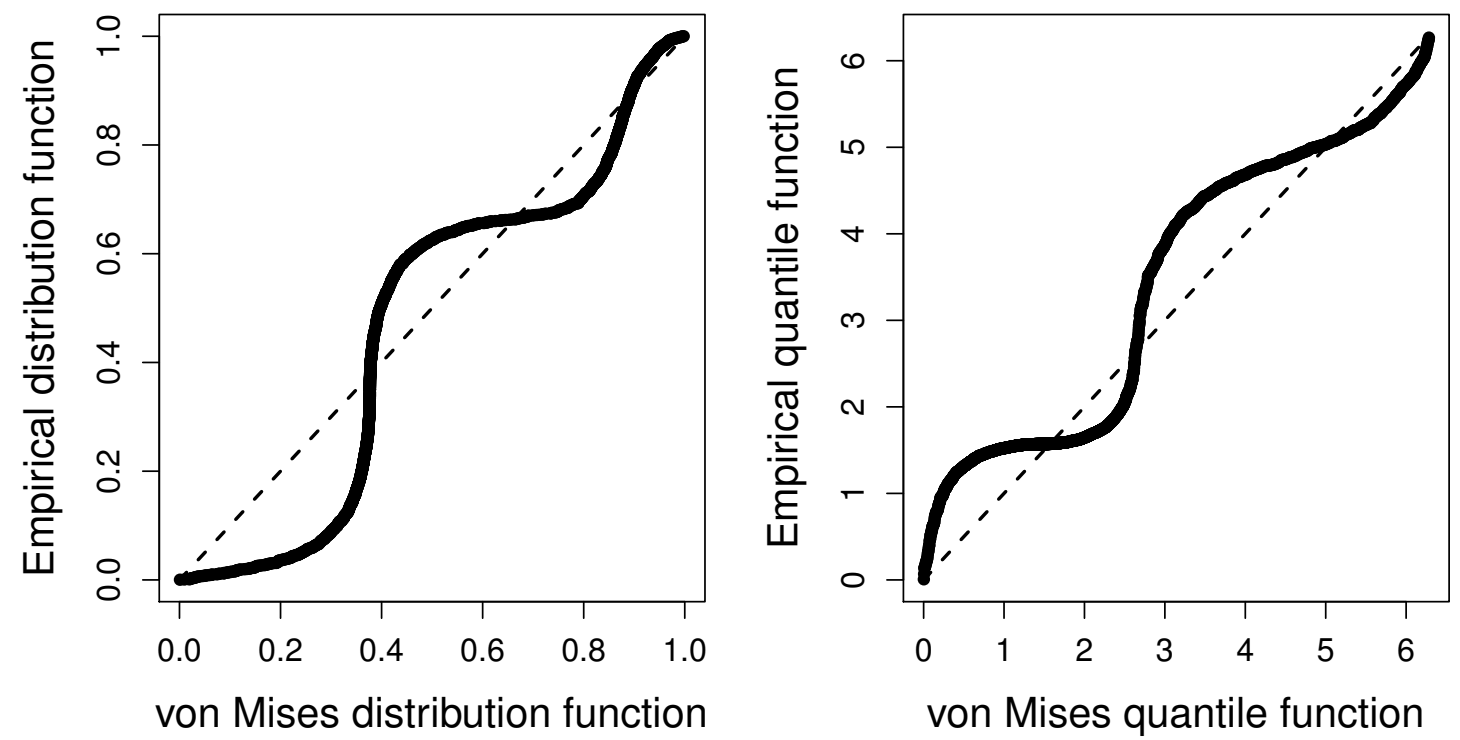

(c) Probability-Probability and Quantile-Quantile plots

Figure 5.2: Theoretical fast polarisations randomly sampled from a plane of vertical cracks striking $045^{\circ}$ with the $Q-Q$ and $P$-P plots for the best fitting von Mises distribution fit to the doubled fast polarisations. 

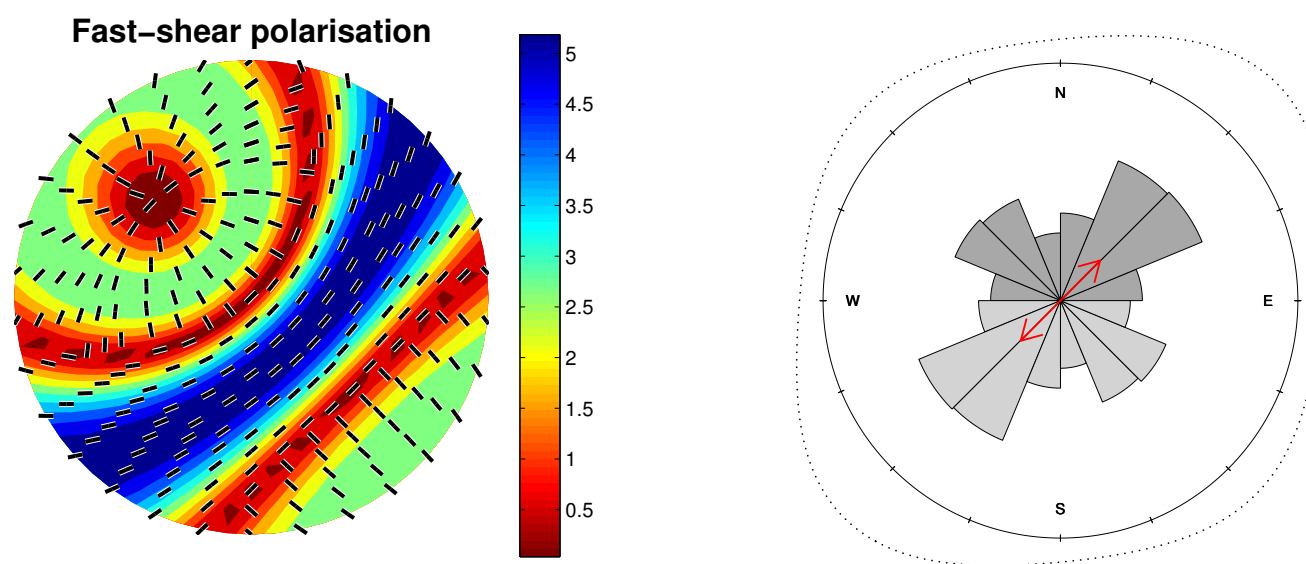

(a) Population of fast polarisations (black

(b) Rose diagram of sampled fast polarisalines)
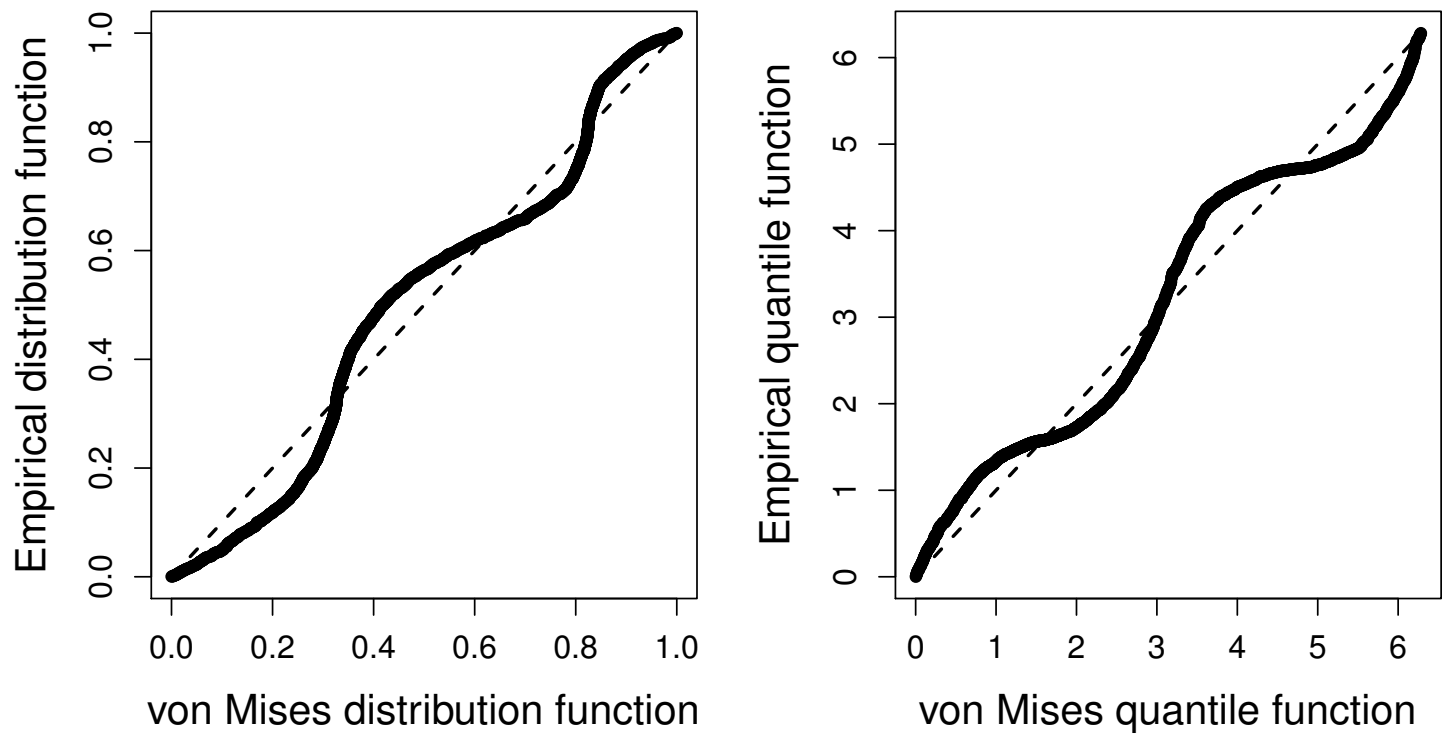

(c) Probability-Probability and Quantile-Quantile plots

Figure 5.3: Theoretical polarisations randomly sampled from a plane of dipping $\left(60^{\circ}\right)$ cracks striking $045^{\circ}$ with the $Q-Q$ and $P-P$ plots for the best fitting von Mises distribution fit to the doubled fast polarisations. 
tribution. Following the approach of Pewsey et al. (2013), we can test the fit of a Jones-Pewsey distribution in the same manner as we did for the von Mises distribution. As can be seen in Figure 5.5, a Jones-Pewsey disruption gives a much better fit to our sampled fast polarisations than a simple von Mises distribution. Depending on the desired outcome, the Jones-Pewsey distribution may be a good candidate fitting to fast polarisations.

We apply this to our own stations to return better estimates of mean fast polarisation. To resolve the $90^{\circ}$ ambiguity, we assume the axis that corresponds to crack strike will be closest to the axis returned from the normal (double angle) method of determining mean (i.e. majority of measurements are parallel to crack strike). We are effectively treating polarisations $90^{\circ}$ to each other as identical to reduce our measured polarisations to a unimodal distribution, and then resolving this ambiguity by assuming that the larger mode corresponds to the strike of the cracks within the anisotropic medium being sampled.

The results are shown in Table 5.4 and Table 5.5 (for those which fail the Rayleigh test). By quadrupling our fast polarisations, five additional stations now pass the Rayleigh test. However, their returned means should be treated with caution. Because they previously failed the Rayleigh test neither mode is dominant so we may not have correctly selected the dominant mode. All but two stations show an improvement in standard error.

In Section 4.3.4 we describe three stations (RT14, RT18, RT19) that fail the Rayleigh test. One reason we suggest for this is steep angles of incidence may be causing switches of $90^{\circ}$ in fast polarisation. Both RT14 and RT8 pass the Rayleigh test for quadrupled polarisations. RT19 does not however, with only 15 polarisations, may not have enough measurements to reach significance. Given this result, polarisations being switch $90^{\circ}$ by some mechanism are likely particuarly present in these three stations. 


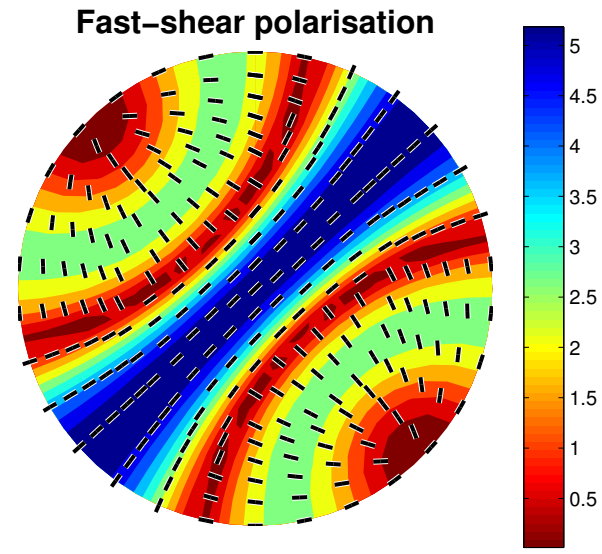

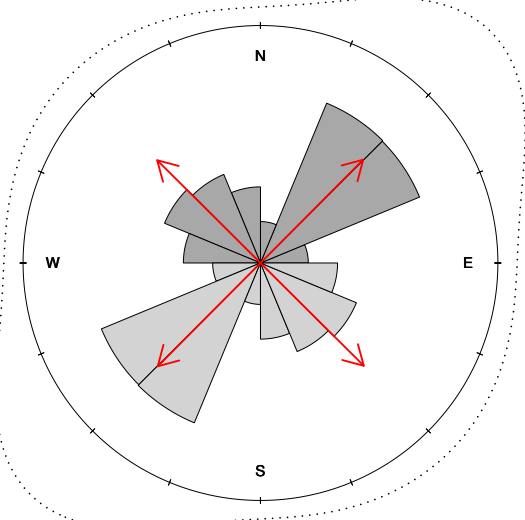

(b) Rose diagram of sampled fast polarisa-

(a) Population of fast polarisations (black tions lines)
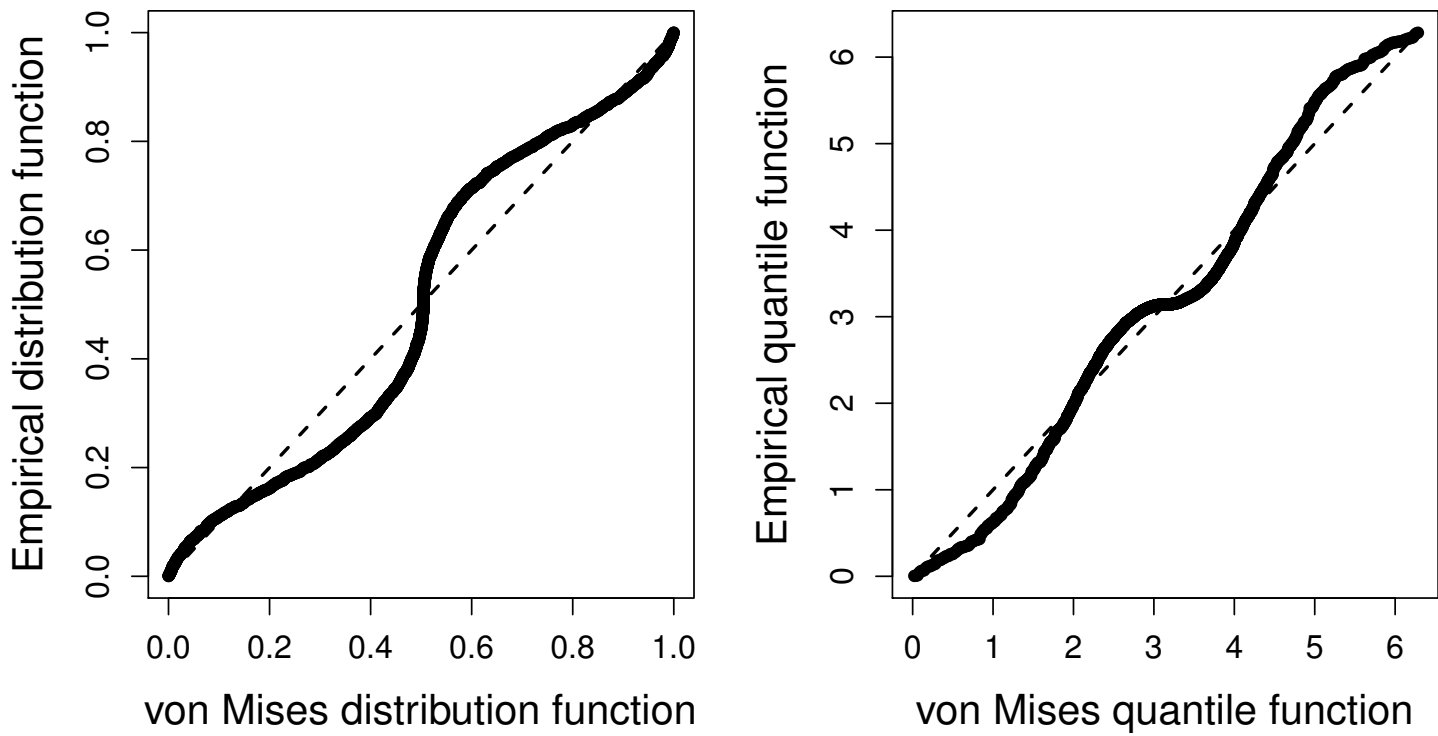

(c) Probability-Probability and Quantile-Quantile plots

Figure 5.4: Theoretical polarisations randomly sampled from a plane of vertical cracks striking $045^{\circ}$ with the $Q-Q$ and $P$-P plots for the best fitting von Mises distribution fit to the quadrupled fast polarisations. a) shows two mean axis to highlight the $90^{\circ}$ ambiguity. 
Table 5.4: Calculated assuming bi-modality, as described in Section 5.6.1. Station means for shear wave splitting measurements for stations with dominant mean orientations (i.e. pass the Rayleigh test with a p-value $\leq$ 0.05). S.E. and S.D. are standard deviation and standard error respectively. Borehole stations are marked with $*$.

\begin{tabular}{|c|c|c|c|c|c|c|c|c|}
\hline Station & $\phi\left(^{\circ}\right)$ & S.D. $\left(^{\circ}\right)$ & S.E. $\left({ }^{\circ}\right)$ & p-value & $\delta t(\mathrm{~s})$ & S.D. $(\mathrm{s})$ & S.E. $(\mathrm{s})$ & No. \\
\hline \hline ARAZ & 48 & 19 & 5.4 & 0.024 & 0.11 & 0.041 & 0.0086 & 23 \\
\hline NS01 & 52 & 25 & 3.9 & $9 \mathrm{e}-04$ & 0.098 & 0.039 & 0.0032 & 147 \\
\hline NS03 & 12 & 24 & 3.6 & 0.00047 & 0.092 & 0.037 & 0.0032 & 134 \\
\hline NS07 & -68 & 19 & 4.7 & 0.0074 & 0.098 & 0.038 & 0.0071 & 29 \\
\hline NS11 & 78 & 20 & 4.9 & 0.0089 & 0.092 & 0.035 & 0.0063 & 31 \\
\hline NS13* & 48 & 23 & 3.7 & 0.00048 & 0.076 & 0.038 & 0.0039 & 97 \\
\hline NS14* & 34 & 23 & 3.6 & 0.00044 & 0.086 & 0.036 & 0.0035 & 108 \\
\hline NS15 & 48 & 23 & 6.2 & 0.04 & 0.08 & 0.04 & 0.0063 & 40 \\
\hline NS16 & 40 & 23 & 3 & $2.2 \mathrm{e}-05$ & 0.072 & 0.053 & 0.0044 & 143 \\
\hline NS18 & 30 & 17 & 4 & 0.0014 & 0.069 & 0.029 & 0.0058 & 25 \\
\hline RT01 & -0.058 & 25 & 2.2 & $3.7 \mathrm{e}-09$ & 0.087 & 0.043 & 0.002 & 453 \\
\hline RT05 & 45 & 29 & 3.5 & 0.00015 & 0.065 & 0.036 & 0.0015 & 570 \\
\hline RT12 & -15 & 19 & 1.5 & $1 \mathrm{e}-17$ & 0.11 & 0.038 & 0.0025 & 228 \\
\hline RT14 & 56 & 19 & 3.4 & 0.00019 & 0.073 & 0.037 & 0.005 & 54 \\
\hline RT17 & 13 & 26 & 3.4 & 0.00015 & 0.094 & 0.034 & 0.0022 & 226 \\
\hline RT18 & 54 & 29 & 5.6 & 0.019 & 0.072 & 0.037 & 0.0023 & 263 \\
\hline RT22 & -35 & 17 & 4.6 & 0.0087 & 0.085 & 0.035 & 0.0084 & 18 \\
\hline WPRZ & 40 & 22 & 2 & $8 \mathrm{e}-12$ & 0.086 & 0.044 & 0.0028 & 245 \\
\hline & & & & & & & & \\
\hline
\end{tabular}



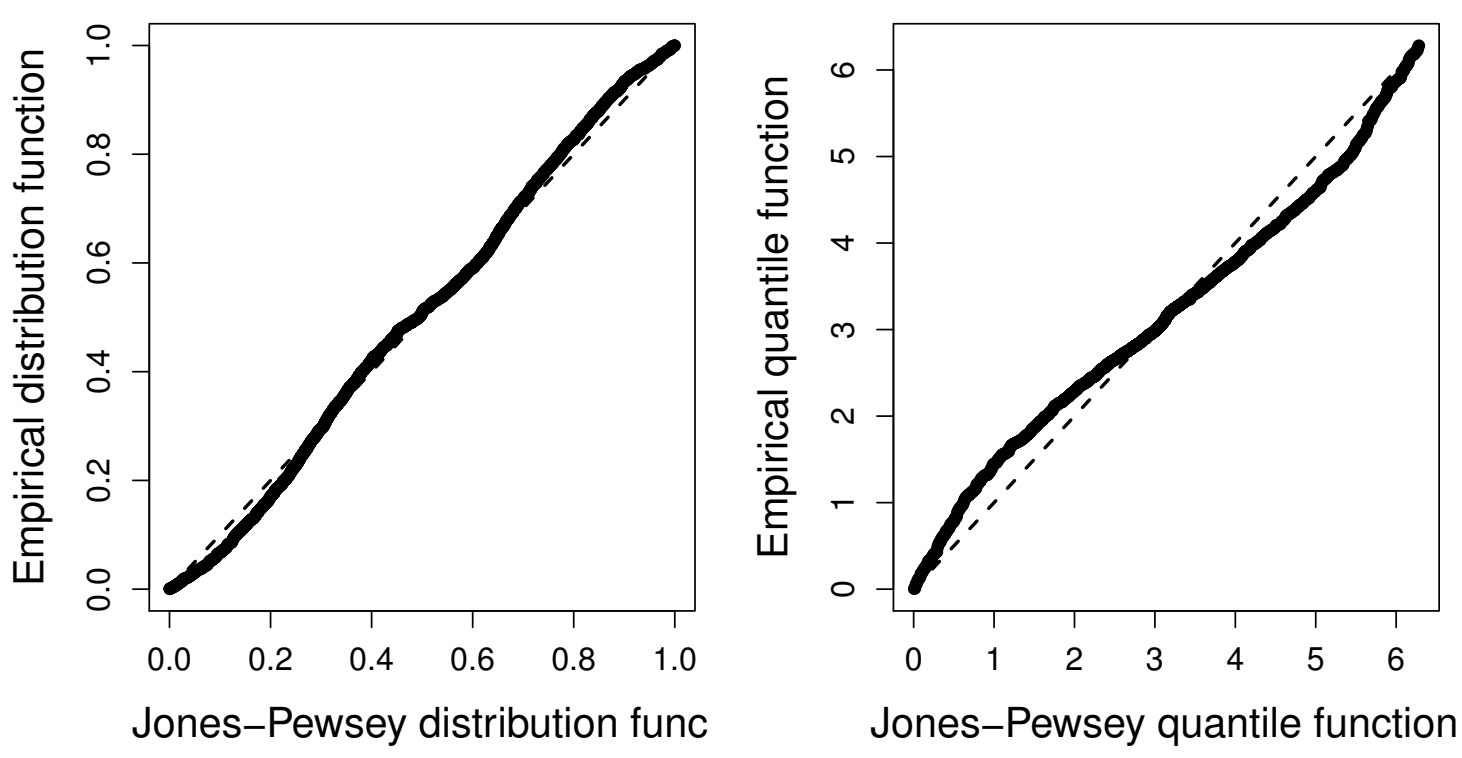

Figure 5.5: $Q-Q$ and $P-P$ plots for the best fitting Jones-Pewsey distribution fit to the quadrupled fast polarisations.

Table 5.5: Calculated assuming bimodality, as described in Section 5.6.1. Station means for shear wave splitting measurements for stations without dominant mean orientations (i.e. fail the Rayleigh test with a p-value > 0.05). S.E. and S.D. are standard deviation and standard error respectively. Borehole stations are marked with $*$.

\begin{tabular}{|c|c|c|c|c|c|c|c|c|}
\hline Station & $\phi\left(^{\circ}\right)$ & S.D. $\left(^{\circ}\right)$ & S.E. $\left(^{\circ}\right)$ & p-value & $\delta t(\mathrm{~s})$ & S.D. (s) & S.E. (s) & No. \\
\hline \hline NS04 & 82 & 1.5 & 1.1 & 0.14 & 0.11 & 0.0018 & 0.0013 & 2 \\
\hline NS12 & -58 & 26 & 8.2 & 0.14 & 0.083 & 0.035 & 0.0046 & 58 \\
\hline RT19 & 49 & 28 & 16 & 0.7 & 0.086 & 0.052 & 0.013 & 15 \\
\hline RT21 & -32 & 22 & 8.5 & 0.17 & 0.11 & 0.039 & 0.009 & 19 \\
\hline RT23 & 1.5 & 22 & 8.9 & 0.21 & 0.11 & 0.025 & 0.0058 & 18 \\
\hline
\end{tabular}




\subsection{Applications}

Ideally shear wave splitting would be able to give remotely measured information about the stress/cracks within both reservoirs which allows changes in the stress state to be monitored especially with respect to field operations. During our study period there are no significant changes in field operations which we can test for differences. General variations through time seem to require the addition of more years to the catalogue.

The framework we have built to apply our methods (some of which are available, see Appendix E.1) can easily be used to investigate an expanded earthquake catalogue. An expanded catalogue will also increase resolution and give more reliable and statistically significant results.

\subsection{Future work}

- Earthquake data from 2012 onwards has recently been made available. Increasing the catalogue with these earthquakes along with matched filter detections will allow:

- Comparisons through time including between periods of differing stimulation where we expect differences.

- An increase measurements will improve the path clustering of earthquakes.

- 3D delay tomography within Rotokawa, where instrument and earthquake density is highest, may become possible.

- Improve estimates of angles of incidence, approximation of propagation angle and earthquake locations by determining a more well defined velocity structure for both fields. 
- Further in-situ stress and crack measurements for existing logs in Rotokawa, and importantly, Ngatamariki can be made to provide a comparison for shear wave splitting parameters. 


\section{Chapter 6}

\section{Conclusions}

We have determined shear wave splitting across the geothermal fields of Rotokawa and Ngatamariki for a dataset of 52,000 unique station to event pairs. We compare our measurements with the orientation of maximum horizontal stress measured regionally (Townend et al. 2012), locally (McNamara et al. 2015), and inferred from active faults (Wallis et al. 2013).

We measure, across both fields, a mean percent anisotropy of approximately $4 \%$ which represents the upper bound of that measured in the upper crust for intact rock (Section 4.3.2).

Fast polarisations measured are consistent with stress aligned microcracks as they largely correspond to the direction of maximum horizontal stress. We also observe a correlation between $\mathrm{P}$-wave to $\mathrm{S}$-wave velocity ratios $\left(v_{P} / v_{S}\right)$ and delay time per kilometre $(\delta t p k m)$ which is consistent with stress aligned microcracks. For saturated cracks (that decrease $v_{S}$ ), increasing crack density (measured by $\delta t p k m$ ) proportionally reduces $v_{S}$ so, if the anisotropy is originating from these cracks, an increase in $v_{P} / v_{S}$ should be proportional to an increase in $\delta t p k m$. 


\subsection{Fitting of crack planes}

The most representative crack planes, with both high uniqueness and low misfit, have constituent cracks that strike $045^{\circ}$ with the regional direction of maximum horizontal stress $\left(S_{H_{m a x}}\right)$ outside of both fields, $035^{\circ}$ in Ngatamariki consistent with strike of active faults, and $035^{\circ}$ through to $0^{\circ}$ within Rotokawa (Section 4.4).

\subsection{Spatial averaging}

Within Ngatamariki, the spatial averaging of fast polarisations largely matches with the strike of faults and is close to the regional direction of $S_{H_{\max }}$ of NE-SW (Section $4.5)$.

The production field fault $(\mathrm{PFF})$, central field fault $(\mathrm{CFF})$, and injection field fault (IFF) are three normal faults that represent major structural features within Rotokawa. Mean fast polarisations for grid cells adjacent to these faults are almost parallel with them, suggesting that either $S_{H_{\max }}$ is rotating locally with the fault or there is anisotropy associated with the faults related fabrics and fractures (e.g. Evans et al. 1995).

\subsection{Delay time tomography}

Delay time tomography (Section 4.5) highlights areas of heightened delay time per kilometre. Both Rotokawa and Ngatamariki exhibit areas of high delay time. Within Rotokawa, the production field fault may be associated with regions of high anisotropy indicating a higher crack density. 


\section{4 $v_{P} / v_{S}$ and cracks}

We measure normally distributed $v_{P} / v_{S}$ across both fields ranging from 1.1 to 2.4 with a mean of 1.6 (Section 4.6). Gas/steam filled cracks exhibit low $v_{P} / v_{S}$ while saturated cracks exhibit high $v_{P} / v_{S}$ (Unglert et al. 2011), thus it appears that most station-earthquake paths have a mixture of both gas filled and saturated cracks along their path. We find a statistically significant positive correlation between $v_{P} / v_{S}$ and $\delta t p k m$, which indicates that the majority of cracks are saturated. Increase in delay time corresponds to an increase in crack density which proportionally increases $v_{P} / v_{S}$. This also provides evidence that the anisotropy being measured does originate from saturated microcracks or this correlation would not be present (Unglert et al. 2011).

\subsection{Restricting the dataset}

The dataset was restricted to only shear wave splitting measurements that should have polarisations parallel with crack strike and consistent delay times (described in Section 3.9.1). The aim was to improve delay time tomography and the spatial averaging of fast polarisation. However, the restriction proved too harsh and greatly reduced the resolution of both these methods by removing too many measurements. So, from the quality of the results, we cannot draw a conclusion as to whether restricting the dataset is having the desired impact.

We do see strong evidence for the restriction working in $v_{P} / v_{S}$. The correlation coefficient between $v_{P} / v_{S}$ and $\delta t p k m$ increases significantly, from 0.07 to 0.26 , between the full dataset to the restricted dataset. This is occurring because the dataset restriction is removing measurements with anomalously low delay time which would otherwise correlate badly with $v_{P} / v_{S}$.

So we have evidence that restricting the dataset is having the desired effect. Delay 
time tomography and spatial averaging can be improved dramatically by including more years to the earthquake catalogue, thus increasing the size of the restricted dataset.

\subsection{Synopsis}

The methods tested and developed in this thesis have been shown to provide meaningful estimates of stress and crack orientation. However, over the time period of the analysis there were no significant perturbations to the reservoirs from production or re-injection. By re-applying these methods to newly available earthquake data (recorded from 2012) which cover periods of significant change in operations, such as the commissioning of the Ngatamariki power station in 2013, we expect to be able to observe how the cracks and stress state within the reservoirs respond to stimulation. Longer term trends can also be investigated. 


\section{Bibliography}

Agostinelli, C. and U. Lund (2013). R package circular: Circular Statistics (version 0.4-7). CA: Department of Environmental Sciences, Informatics and Statistics, Ca' Foscari University, Venice, Italy. UL: Department of Statistics, California Polytechnic State University, San Luis Obispo, California, USA.

Akaike, H. (1973). "Information theory and an extension of the maximum likelihood principle". In: Second International Symposium on Information Theory, pp. 267281.

Akima, H. and A. Gebhardt (2015). akima: Interpolation of Irregularly and Regularly Spaced Data. R package version 0.5-12.

Allen, R. V. (1978). "Automatic earthquake recognition and timing from single traces". In: Bulletin of the Seismological Society of America 68.5, pp. 1521-1532.

Anton, H. and R. C. Busby (2002). Contemporary Linear Algebra. John Wiley \& Sons, Inc.

Babuska, V. and M. Cara (1991). Seismic anisotropy in the Earth. Vol. 10. Springer Science \& Business Media.

Baker, G. E. and J. L. Stevens (2004). "Backazimuth estimation reliability using surface wave polarization". In: Geophysical research letters 31.9.

Banerjee, A., I. S. Dhillon, J. Ghosh, and S. Sra (2005). "Clustering on the unit hypersphere using von Mises-Fisher distributions". In: Journal of Machine Learning Research, pp. 1345-1382.

Booth, D. C. and S. Crampin (1985). "Shear-wave polarizations on a curved wavefront at an isotropic free surface". In: Geophysical Journal International 83.1, pp. 31-45.

Boseley, C., G. Bignall, A. Rae, I. Chambefort, and B. Lewis (2012). "Stratigraphy and hydrothermal alteration encountered by monitor wells complete at Ngatamariki and Orakei Korako in 2011". In: Proceedings of the $34^{\text {th }}$ New Zealand geothermal workshop. Auckland, New Zealand. 
Boseley, C., W. Cumming, L. Urzúa-Monsalve, T. Powell, and M. Grant (2010). "A resource conceptual model for the Ngatamariki geothermal field based on recent exploration well drilling and 3D MT resistivity imaging". In: Proceedings World Geothermal Congress.

Bower, A. F. (2009). Applied mechanics of solids. CRC press.

Bowyer, D. and R. Holt (2010). "Case study: Development of a numerical model by a multi-disciplinary approach, Rotokawa Geothermal Field, New Zealand". In: Proceedings World Geothermal Congress 2010, pp. 25-29.

Budiansky, B. and R. J. O'connell (1976). "Elastic moduli of a cracked solid". In: International journal of Solids and structures 12.2, pp. 81-97.

Buscarlet, E., H. Moon, I. Wallis, and J. Quinao (2015). "Reservoir tracer test at the Ngatamariki geothermal field". In: Proc. NZ Geothermal Workshop.

Carey, B., M. Dunstall, S. McClintock, B. White, G. Bignall, K. Luketina, B. Robson, S. Zarrouk, and A. Seward (2015). "2015 New Zealand country update". In: Proceeding, World Geothermal Congress, p. 16.

Cassidy, J. F. and M. G. Bostock (1996). "Shear-wave splitting above the subducting Juan de Fuca Plate". In: Geophysical Research Letters 23.9, pp. 941-944.

Castellazzi, C., M. K. Savage, E. Walsh, and R. Arnold (2015). "Shear wave automatic picking and splitting measurements at Ruapehu volcano, New Zealand". In: Journal of Geophysical Research. Solid Earth 120.5, pp. 3363-3384.

Chambefort, I., B. Lewis, C. Wilson, A. Rae, C. Coutts, G. Bignall, and T. Ireland (2014). "Stratigraphy and structure of the Ngatamariki geothermal system from new zircon $\mathrm{U}-\mathrm{Pb}$ geochronology: Implications for Taupo Volcanic Zone evolution". In: Journal of Volcanology and Geothermal Research 274, pp. 51-70.

Chambefort, I., E. Buscarlet, I. C. Wallis, S. Sewell, and M. Wilmarth (2016). "Ngatamariki Geothermal Field, New Zealand: Geology, geophysics, chemistry and conceptual model". In: Geothermics 59, pp. 266-280.

Chamberlain, C. J., D. R. Shelly, J. Townend, and T. A. Stern (2014). "Lowfrequency earthquakes reveal punctuated slow slip on the deep extent of the Alpine Fault, New Zealand". In: Geochemistry, Geophysics, Geosystems 15.7, pp. 2984-2999.

Chapman, M. (2003). "Frequency-dependent anisotropy due to meso-scale fractures in the presence of equant porosity". In: Geophysical Prospecting 51.5, pp. 369379 . 
Cichowicz, A. (1993). "An automatic S-phase picker". In: Bulletin of the Seismological Society of America 83.1, pp. 180-189.

Civilini, F., A. Pancha, M. K. Savage, S. Sewell, and J. Townend (2016). "Inferring shear-velocity structure of the upper $200 \mathrm{~m}$ using cultural ambient noise at the Ngatamariki geothermal field, Central North Island, New Zealand". In: Interpretation 4.3, SJ87-SJ101.

Clacy, G. (1968). "Geothermal ground noise amplitude and frequency spectra in the New Zealand volcanic region". In: Journal of Geophysical Research 73.16, pp. 5377-5383.

Cole, J. and K. Lewis (1981). "Evolution of the Taupo-Hikurangi subduction system". In: Tectonophysics 72.1-2, pp. 1-21.

Crampin, S., R. Evans, and B. K. Atkinson (1984). "Earthquake prediction: a new physical basis". In: Geophysical Journal International 76.1, pp. 147-156.

Crampin, S. (1984). "Effective anisotropic elastic constants for wave propagation through cracked solids". In: Geophysical Journal International 76.1, pp. 135-145.

Crampin, S. (1989). "Suggestions for a consistent terminology for seismic anisotropy". In: Geophysical Prospecting 37.7, pp. 753-770.

Crampin, S. (1999). "Calculable fluid-rock interactions". In: Journal of the Geological Society 156.3, pp. 501-514.

Crampin, S. and D. C. Booth (1985). "Shear-wave polarizations near the North Anatolian Fault-II. Interpretation in terms of crack-induced anisotropy". In: Geophysical Journal International 83.1, pp. 75-92.

Crampin, S. and J. H. Lovell (1991). "A decade of shear-wave splitting in the Earth's crust: what does it mean? what use can we make of it? and what should we do next?" In: Geophysical Journal International 107.3, pp. 387-407.

Crampin, S. and S. Peacock (2005). "A review of shear-wave splitting in the compliant crack-critical anisotropic Earth". In: Wave motion 41.1, pp. 59-77.

Crampin, S. and S. Peacock (2008). "A review of the current understanding of seismic shear-wave splitting in the Earth's crust and common fallacies in interpretation". In: Wave Motion 45.6, pp. 675-722.

Crampin, S. and S. V. Zatsepin (1997). "Modelling the compliance of crustal rockII. Response to temporal changes before earthquakes". In: Geophysical Journal International 129.3 , pp. 495-506. 
Crotwell, H. P., T. J. Owens, and J. Ritsema (1999). "The TauP Toolkit: Flexible seismic travel-time and ray-path utilities". In: Seismological Research Letters 70.2 , pp. $154-160$.

Diehl, T., N. Deichmann, E. Kissling, and S. Husen (2009). "Automatic S-wave picker for local earthquake tomography". In: Bulletin of the Seismological Society of America 99.3, pp. 1906-1920.

Elkibbi, M., M. Yang, and J. A. Rial (2005). "Crack-induced anisotropy models in The Geysers geothermal field". In: Geophysical Journal International 162.3, pp. 1036-1048.

Evans, J. R., B. R. Julian, G. R. Foulger, and A. Ross (1995). "Shear-wave splitting from local earthquakes at The Geysers geothermal field, California". In: Geophysical Research Letters 22.4, pp. 501-504.

Fisher, N. I. (1995). Statistical analysis of circular data. Cambridge University Press.

Gerst, A. and M. K. Savage (2004). "Seismic anisotropy beneath Ruapehu volcano: a possible eruption forecasting tool". In: Science 306.5701, pp. 1543-1547.

Gibbons, S. J. and F. Ringdal (2006). "The detection of low magnitude seismic events using array-based waveform correlation". In: Geophysical Journal International 165.1, pp. 149-166.

Greenbank, E. (2014). "Seismic anisotropy and fractures at Rotokawa Geothermal Field". Unpublished manuscript.

Heise, W., T. Caldwell, H. M. Bibby, and S. Bannister (2008). "Three-dimensional modelling of magnetotelluric data from the Rotokawa geothermal field, Taupo Volcanic Zone, New Zealand". In: Geophysical Journal International 173.2, pp. 740750 .

Hernandez, D., J. Clearwater, J. Burnell, P. Franz, L. Azwar, and A. Marsh (2015). "Update on the modeling of the Rotokawa Geothermal System: 2010-2014". In: Proceedings World Geothermal Congress 2015. Vol. 19, p. 25.

Hopp, C., M. Savage, J. Townend, and S. Sherburn (2016). "Matched filter earthquake detection and double difference relocation at Rotokawa and Ngatamariki geothermal areas: January to November, 2015". In: 38th New Zealand Geothermal Workshop: 2016 Proceedings, Auckland Univ., Auckland, New Zealand.

Hornik, K. and B. Grün (2014). "movMF: An R Package for Fitting Mixtures of von Mises-Fisher Distributions". In: Journal of Statistical Software 58.10, pp. 1-31. 
Hudson, J. (1980). "Overall properties of a cracked solid". In: Mathematical Proceedings of the Cambridge Philosophical Society. Vol. 88. 02. Cambridge Univ Press, pp. 371-384.

Hudson, J. (1981). "Wave speeds and attenuation of elastic waves in material containing cracks". In: Geophysical Journal International 64.1, pp. 133-150.

Johnson, J. H. and M. K. Savage (2012). "Tracking volcanic and geothermal activity in the Tongariro Volcanic Centre, New Zealand, with shear wave splitting tomography". In: Journal of Volcanology and Geothermal Research 223, pp. 1-10.

Johnson, J. H., M. K. Savage, and J. Townend (2011). "Distinguishing between stressinduced and structural anisotropy at Mount Ruapehu Volcano, New Zealand". In: Journal of Geophysical Research: Solid Earth 116.B12.

Johnson, J. H. (2011). "Discriminating between spatial and temporal variations in seismic anisotropy at active volcanoes". PhD thesis. Victoria University of Wellington.

Kahle, D. and H. Wickham (2013). "ggmap: Spatial Visualization with ggplot2". In: The $R$ Journal 5.1, pp. 144-161.

Kitagawa, G. and H. Akaike (1978). "A procedure for the modeling of non-stationary time series". In: Annals of the Institute of Statistical Mathematics 30.1, pp. 351363.

Krupp, R. and T. Seward (1987). "The Rotokawa geothermal system, New Zealand; an active epithermal gold-depositing environment". In: Economic Geology 82.5, pp. 1109-1129.

Leonard, M. and B. Kennett (1999). "Multi-component autoregressive techniques for the analysis of seismograms". In: Physics of the Earth and Planetary Interiors 113.1, pp. 247-263.

Lodewyk, J. and D. Sumy (2015). Cascadia Amphibious Array Ocean Bottom Seismograph Horizontal Component Orientations. Tech. rep. Incorporated Research Institutions for Seismology.

Lomax, A., A. Michelini, and A. Curtis (2014). "Earthquake Location, Direct, GlobalSearch Methods". In:

Lou, M., E. Shalev, and P. Malin (1997). "Shear-wave splitting and fracture alignments at the Northwest Geysers, California". In: Geophysical research letters 24.15 , pp. $1895-1898$. 
Lou, M. and J. A. Rial (1997). "Characterization of geothermal reservoir crack patterns using shear-wave splitting". In: Geophysics 62.2, pp. 487-494.

Love, A. (1927). A Treatise on the Mathematical Theory of Elasticity. Dover Publications, New York.

Mardia, K. V. and P. E. Jupp (2009). Directional statistics. Vol. 494. John Wiley \& Sons.

Marson-Pidgeon, K. and M. K. Savage (1997). "Frequency-dependent anisotropy in Wellington, New Zealand". In: Geophysical Research Letters 24, pp. 3297-3300.

Mason, I. G., S. C. Page, and A. G. Williamson (2010). "A 100\% renewable electricity generation system for New Zealand utilising hydro, wind, geothermal and biomass resources". In: Energy Policy 38.8, pp. 3973-3984.

Massiot, C., D. D. McNamara, A. Nicol, and J. Townend (2015). "Fracture Width and Spacing Distributions from Borehole Televiewer Logs and Cores in the Rotokawa Geothermal Field, New Zealand". In: Proceedings World Geothermal Congress 2015.

Maultzsch, S., M. Chapman, E. Liu, and X. Y. Li (2003). "Modelling frequencydependent seismic anisotropy in fluid-saturated rock with aligned fractures: implication of fracture size estimation from anisotropic measurements". In: Geophysical Prospecting 51.5, pp. 381-392.

McLachlan, G. and D. Peel (2004). Finite mixture models. John Wiley \& Sons.

McNamara, D. D., C. Massiot, B. Lewis, and I. C. Wallis (2015). "Heterogeneity of structure and stress in the Rotokawa Geothermal Field, New Zealand". In: Journal of Geophysical Research: Solid Earth 120.2, pp. 1243-1262.

McNamara, D. D., S. Sewell, E. Buscarlet, and I. C. Wallis (2016). "A review of the Rotokawa Geothermal Field, New Zealand". In: Geothermics 59, pp. 281-293.

Moon, T. K. (1996). "The expectation-maximization algorithm". In: IEEE Signal processing magazine 13.6, pp. 47-60.

Mroczek, S., M. Savage, C. Hopp, and S. Sewell (2016). "Shear Wave Splitting at Rotokawa and Ngatamariki Geothermal Fields for 2015". In: 38th New Zealand Geothermal Workshop: 2016 Proceedings, Auckland Univ., Auckland, New Zealand.

Muksin, U., K. Bauer, and C. Haberland (2013). "Seismic Vp and Vp/Vs structure of the geothermal area around Tarutung (North Sumatra, Indonesia) derived from local earthquake tomography". In: Journal of Volcanology and Geothermal Research 260, pp. 27-42. 
Neuwirth, E. (2014). RColorBrewer: ColorBrewer Palettes. R package version 1.1-2.

Nistala, S. and G. A. McMechan (2005). "3D modeling of fracture-induced shearwave splitting in the southern California basin". In: Bulletin of the Seismological Society of America 95.3, pp. 1090-1100.

Nur, A. (1972). "Dilatancy, pore fluids, and premonitory variations of $t_{s} / t_{p}$ travel times". In: Bulletin of the Seismological society of America 62.5, pp. 1217-1222.

Nuttli, O. (1961). "The effect of the Earth's surface on the S wave particle motion". In: Bulletin of the Seismological Society of America 51.2, pp. 237-246.

Nychka, D., R. Furrer, J. Paige, and S. Sain (2015). fields: Tools for spatial data. $\mathrm{R}$ package version 8.4-1. Boulder, CO, USA: University Corporation for Atmospheric Research.

Peacock, S., S. Crampin, D. C. Booth, and J. B. Fletcher (1988). "Shear wave splitting in the Anza seismic gap, southern California: temporal variations as possible precursors". In: Journal of Geophysical Research: Solid Earth 93.B4, pp. 33393356 .

Pewsey, A., M. Neuhäuser, and G. D. Ruxton (2013). Circular statistics in R. Oxford University Press.

R Core Team (2013). R: A Language and Environment for Statistical Computing. R Foundation for Statistical Computing. Vienna, Austria.

Rawlinson, Z. (2011). "Microseismicity associated with actively exploited geothermal systems: earthquake detection and probabilistic location at Rotokawa and statistical seismic network design at Kawerau". MA thesis. Victoria University of Wellington.

Rial, J. A., M. Elkibbi, and M. Yang (2005). "Shear-wave splitting as a tool for the characterization of geothermal fractured reservoirs: Lessons learned". In: Geothermics 34.3, pp. 365-385.

Risk, G. (2000). "Electrical resistivity surveys of the Rotokawa geothermal field, New Zealand". In: Proceedings of the 22 ${ }^{\text {nd }}$ New Zealand Geothermal Workshop, pp. 121-126.

Roman, D. C., M. K. Savage, R. Arnold, J. L. Latchman, and S. De Angelis (2011). "Analysis and forward modeling of seismic anisotropy during the ongoing eruption of the Soufrière Hills Volcano, Montserrat, 1996-2007". In: Journal of Geophysical Research, Solid Earth 116.B3. 
Rümpker, G. and P. G. Silver (2000). "Calculating splitting parameters for plumetype anisotropic structures of the upper mantle". In: Geophysical Journal International 143.3, pp. 507-520.

Rybach, L., T. Megel, and W. Eugster (2000). "At what time scale are geothermal resources renewable". In: Proc. World Geothermal Congress 2000. Vol. 2, pp. 867873.

Savage, M. K. (1999). "Seismic anisotropy and mantle deformation: What have we learned from shear wave splitting?" In: Reviews of Geophysics 37.1, p. 65.

Savage, M. K., A. Wessel, N. A. Teanby, and A. W. Hurst (2010a). "Automatic measurement of shear wave splitting and applications to time varying anisotropy at Mount Ruapehu volcano, New Zealand". In: Journal of Geophysical Research, Solid Earth 115.12.

Savage, M. K., T. Ohminato, Y. Aoki, H. Tsuji, and S. M. Greve (2010b). "Stress magnitude and its temporal variation at Mt. Asama Volcano, Japan, from seismic anisotropy and GPS". In: Earth and Planetary Science Letters 290.3, pp. 403414.

Sherburn, S. and S. Bourguignon (2014). Ngatamariki Seismic Network and Seismicity - Annual Report 2013. Tech. rep. GNS Science.

Sherburn, S., S. Sewell, S. Bourguignon, W. Cumming, S. Bannister, C. Bardsley, J. Winick, J. Quinao, and I. Wallis (2015). "Microseismicity at Rotokawa geothermal field, New Zealand, 2008-2012". In: Geothermics 54, pp. 23-34.

Silver, P. G. (1996). "Seismic anisotropy beneath the continents: Probing the depths of geology". In: Annual review of earth and planetary sciences 24, pp. 385-432.

Silver, P. G. and W. W. Chan (1991). "Shear wave splitting and subcontinental mantle deformation". In: Journal of Geophysical Research: Solid Earth 96.B10, pp. 16429-16454.

Siratovich, P. A., M. J. Heap, M. C. Villenueve, J. W. Cole, and T. Reuschlé (2014). "Physical property relationships of the Rotokawa Andesite, a significant geothermal reservoir rock in the Taupo Volcanic Zone, New Zealand". In: Geothermal Energy 2.1, p. 10.

Stachnik, J., A. F. Sheehan, D. Zietlow, Z. Yang, J. Collins, and A. Ferris (2012). "Determination of New Zealand ocean bottom seismometer orientation via Rayleighwave polarization". In: Seismological Research Letters 83.4, pp. 704-713.

Stein, S. and M. Wysession (2009). An introduction to seismology, earthquakes, and earth structure. John Wiley \& Sons. 
Stern, T. (1987). "Asymmetric back-arc spreading, heat flux and structure associated with the Central Volcanic Region of New Zealand". In: Earth and planetary science letters 85.1, pp. 265-276.

Syracuse, E., C. Thurber, C. Rawles, M. Savage, and S. Bannister (2013). "Highresolution relocation of aftershocks of the Mw 7.1 Darfield, New Zealand, earthquake and implications for fault activity". In: Journal of Geophysical Research: Solid Earth 118.8, pp. 4184-4195.

Takanami, T. and G. Kitagawa (1988). "A new efficient procedure for the estimation of onset times of seismic waves." In: Journal of Physics of the Earth 36.6, pp. 267290 .

Teanby, N., J.-M. Kendall, and M. Van der Baan (2004). "Automation of shear-wave splitting measurements using cluster analysis". In: Bulletin of the Seismological Society of America 94.2, pp. 453-463.

Townend, J., S. Sherburn, R. Arnold, C. Boese, and L. Woods (2012). "Threedimensional variations in present-day tectonic stress along the Australia-Pacific plate boundary in New Zealand". In: Earth and Planetary Science Letters 353, pp. $47-59$.

Townend, J. and M. D. Zoback (2001). "Implications of earthquake focal mechanisms for the frictional strength of the San Andreas fault system". In: Geological Society, London, Special Publications 186.1, pp. 13-21.

Uhrhammer, R. A. and E. R. Collins (1990). "Synthesis of Wood-Anderson seismograms from broadband digital records". In: Bulletin of the Seismological Society of America 80.3, pp. 702-716.

Unglert, K., M. Savage, N. Fournier, T. Ohkura, and Y. Abe (2011). "Shear wave splitting, $v_{P} / v_{S}$, and GPS during a time of enhanced activity at Aso caldera, Kyushu". In: Journal of Geophysical Research, Solid Earth 116.B11.

Unglert, K. (2009). "Shear-wave splitting for monitoring Rotokawa Geothermal Field, New Zealand: A feasibility study". Unpublished manuscript.

Volti, T. and S. Crampin (2003). "A four-year study of shear-wave splitting in Iceland: 1. Background and preliminary analysis". In: Geological Society, London, Special Publications 212.1, pp. 117-133.

Vucetich, C. and R. Howorth (1976). "Proposed definition of the Kawakawa Tephra, the c. 20 000-years-BP marker horizon in the New Zealand region". In: New Zealand journal of geology and geophysics 19.1, pp. 43-50. 
Walker, A. M. and J. Wookey (2012). "MSAT-A new toolkit for the analysis of elastic and seismic anisotropy". In: Computers $\&$ Geosciences 49, pp. 81-90.

Wallace, L. M., J. Beavan, R. McCaffrey, and D. Darby (2004). "Subduction zone coupling and tectonic block rotations in the North Island, New Zealand". In: Journal of Geophysical Research: Solid Earth 109.B12.

Wallis, I., C. Bardsley, T. Powell, J. Rowland, and J. O'Brien (2013). "A structural model for the Rotokawa geothermal field, New Zealand". In: 35th New Zealand Geothermal Workshop: 2013 Proceedings, Auckland Univ., Auckland, New Zealand.

Walsh, E., R. Arnold, and M. K. Savage (2013). "Silver and Chan revisited". In: Journal of Geophysical Research. Solid Earth 118.10, pp. 5500-5515.

Watson, G. S., G. S. Watson, M. Statistiker, G. S. Watson, P. Statisticien, E.-U. Australie, and G. S. Watson (1983). Statistics on spheres. Vol. 3. Wiley New York.

Wessel, A. (2010). "Shear Wave Splitting Measurements at Mt. Ruapehu Volcano, New Zealand". MSc thesis. Victoria University of Wellington.

Wessel, A., M. Savage, and N. Teanby (2016). Manual for the Multiple Filter Automatic Splitting Technique (MFAST) processing codes, Version 2.2. Victoria University of Wellington.

Wickham, H. (2009). ggplot2: Elegant Graphics for Data Analysis. Springer-Verlag New York.

Wilkie, D. (1983). "Rayleigh test for randomness of circular data". In: Applied statistics. Citeseer.

Wilson, C., B. Houghton, M. McWilliams, M. Lanphere, S. Weaver, and R. Briggs (1995). "Volcanic and structural evolution of Taupo Volcanic Zone, New Zealand: a review". In: Journal of volcanology and geothermal research 68.1, pp. 1-28.

Wilson, C. J. and J. V. Rowland (2016). "The volcanic, magmatic and tectonic setting of the Taupo Volcanic Zone, New Zealand, reviewed from a geothermal perspective". In: Geothermics 59, pp. 168-187.

Zhang, Z. and S. Y. Schwartz (1994). "Seismic anisotropy in the shallow crust of the Loma Prieta segment of the San Andreas fault system". In: Journal of Geophysical Research: Solid Earth 99.B5, pp. 9651-9661.

Zinke, J. C. and M. D. Zoback (2000). "Structure-related and stress-induced shearwave velocity anisotropy: observations from microearthquakes near the Calaveras 
Fault in Central California". In: Bulletin of the Seismological Society of America 90.5, pp. 1305-1312. 
Appendix A

\section{Tables}


Table A.1: Teleseismic events used for borehole station orientation from 2014. From the USGS catalogue with depth less that $100 \mathrm{~km}$, magnitude greater than 6, and epicentral distance greater than $30^{\circ}$.

\begin{tabular}{|c|c|c|c|c|c|}
\hline Date & Latitude & Longitude & Depth $(\mathrm{km})$ & Magnitude & USGS ID \\
\hline \hline $2014-03-16$ & -19.9807 & -70.7022 & 20 & 6.7 & usc000ndnj \\
\hline $2014-04-03$ & -20.7969 & -70.5865 & 25 & 6.4 & usc000p2cs \\
\hline $2014-04-03$ & -20.5709 & -70.4931 & 22.4 & 7.7 & usc000p27i \\
\hline $2014-04-03$ & -20.3113 & -70.5756 & 24.07 & 6.5 & usc000p26f \\
\hline $2014-04-11$ & -6.5858 & 155.0485 & 60.53 & 7.1 & usc000pft9 \\
\hline $2014-04-12$ & -11.2701 & 162.1481 & 22.56 & 7.6 & usc000phx5 \\
\hline $2014-04-18$ & 17.397 & -100.9723 & 24 & 7.2 & usb000pq41 \\
\hline $2014-04-19$ & -6.7547 & 155.0241 & 43.37 & 7.5 & usb000pr89 \\
\hline $2014-04-19$ & -6.6558 & 155.0869 & 29 & 6.6 & usb000pqwe \\
\hline $2014-04-20$ & -7.1646 & 155.3351 & 20 & 6.2 & usb000prqv \\
\hline $2014-05-12$ & -49.9403 & -114.7995 & 10.47 & 6.5 & usb000qjhh \\
\hline $2014-06-29$ & -55.4703 & -28.3669 & 8 & 6.9 & usc000rngj \\
\hline $2014-08-23$ & -32.6953 & -71.4416 & 32 & 6.4 & usb000s5rc \\
\hline $2014-09-25$ & -9.4618 & 156.4122 & 4 & 6.1 & usb000sftz \\
\hline $2014-10-09$ & -32.1082 & -110.8112 & 16.54 & 7 & usb000sk6k \\
\hline $2014-10-14$ & 12.5262 & -88.1225 & 40 & 7.3 & usb000slwn \\
\hline $2014-11-26$ & 1.9604 & 126.5751 & 39 & 6.8 & usb000t08w \\
\hline $2014-12-07$ & -6.5108 & 154.4603 & 23 & 6.6 & usc000t4cz \\
\hline
\end{tabular}


Table A.2: Teleseismic events used for borehole station orientation from 2015. From the USGS catalogue with depth less that $100 \mathrm{~km}$, magnitude greater than 6, and epicentral distance greater than $30^{\circ}$.

\begin{tabular}{|c|c|c|c|c|c|}
\hline Date & Latitude & Longitude & Depth $(\mathrm{km})$ & Magnitude & USGS ID \\
\hline \hline $2015-05-05$ & -5.4624 & 151.8751 & 55 & 7.5 & us20002bnf \\
\hline $2015-05-07$ & -7.2175 & 154.5567 & 10 & 7.1 & us20002das \\
\hline $2015-05-12$ & 38.9056 & 142.0317 & 35 & 6.8 & us20002et4 \\
\hline $2015-05-19$ & -54.3312 & -132.1618 & 7.2 & 6.7 & us10002aj3 \\
\hline $2015-07-10$ & -9.307 & 158.403 & 12 & 6.7 & us20002whh \\
\hline $2015-07-27$ & -2.6286 & 138.5277 & 48 & 7 & us200030kn \\
\hline $2015-07-27$ & 52.376 & -169.4458 & 29 & 6.9 & us200030aq \\
\hline $2015-08-10$ & -9.3438 & 158.0525 & 22 & 6.6 & us100030pg \\
\hline $2015-08-12$ & -9.3293 & 157.8772 & 6.43 & 6.5 & us100031me \\
\hline $2015-09-16$ & -31.618 & -71.745 & 26.7 & 6.4 & us20003k8b \\
\hline $2015-09-16$ & -31.5729 & -71.6744 & 22.44 & 8.3 & us20003k7a \\
\hline $2015-09-17$ & -31.5173 & -71.804 & 23 & 6.7 & us20003kfv \\
\hline $2015-09-21$ & -31.7275 & -71.3792 & 35 & 6.6 & us20003mi0 \\
\hline $2015-09-24$ & -0.6212 & 131.2622 & 18 & 6.6 & us20003nqr \\
\hline $2015-11-04$ & -8.3381 & 124.8754 & 20 & 6.5 & us200041ty \\
\hline $2015-11-18$ & -8.8994 & 158.4217 & 12.59 & 6.8 & us10003zcp \\
\hline
\end{tabular}


Table A.3: Numbers of measurements for each station. norm $/ n$ indicates event was part of GNS catalogue, det/d indicates the event was a matched filter detection and tot/t indicates a total. pick are the numbers of events successfully picked and sws are the number of high grade shear wave splitting measurements. Borehole stations are marked with $*$.

\begin{tabular}{|c|c|c|c|c|c|c|c|c|c|}
\hline Station & norm & det & tot & n_pick & d_pick & t_pick & n_sws & d_sws & t_sws \\
\hline \hline ARAZ & 810 & 961 & 1771 & 77 & 69 & 146 & 14 & 9 & 23 \\
\hline NS01 & 1305 & 1697 & 3002 & 270 & 255 & 525 & 80 & 67 & 147 \\
\hline NS03 & 806 & 894 & 1700 & 254 & 138 & 392 & 87 & 47 & 134 \\
\hline NS07 & 586 & 727 & 1313 & 97 & 41 & 138 & 23 & 6 & 29 \\
\hline NS08 & 57 & 33 & 90 & 8 & 1 & 9 & 0 & 0 & 0 \\
\hline NS09 & 218 & 129 & 347 & 28 & 1 & 29 & 0 & 0 & 0 \\
\hline NS11 & 653 & 983 & 1636 & 76 & 50 & 126 & 17 & 14 & 31 \\
\hline NS12* & 921 & 1337 & 2258 & 130 & 73 & 203 & 42 & 16 & 58 \\
\hline NS13* & 661 & 1065 & 1726 & 155 & 100 & 255 & 73 & 24 & 97 \\
\hline NS14* & 990 & 1534 & 2524 & 169 & 165 & 334 & 50 & 58 & 108 \\
\hline NS15 & 610 & 509 & 1119 & 94 & 42 & 136 & 28 & 12 & 40 \\
\hline NS16 & 939 & 1145 & 2084 & 191 & 127 & 318 & 84 & 59 & 143 \\
\hline NS18 & 524 & 783 & 1307 & 43 & 27 & 70 & 13 & 12 & 25 \\
\hline RT01 & 1782 & 2042 & 3824 & 579 & 452 & 1031 & 257 & 196 & 453 \\
\hline RT05 & 1526 & 1707 & 3233 & 528 & 509 & 1037 & 281 & 289 & 570 \\
\hline RT12 & 1255 & 1487 & 2742 & 301 & 278 & 579 & 117 & 111 & 228 \\
\hline RT14 & 1241 & 1638 & 2879 & 102 & 96 & 198 & 32 & 22 & 54 \\
\hline RT17 & 1393 & 1628 & 3021 & 339 & 269 & 608 & 120 & 106 & 226 \\
\hline RT18 & 1436 & 1822 & 3258 & 353 & 333 & 686 & 139 & 124 & 263 \\
\hline RT19 & 1438 & 1771 & 3209 & 18 & 15 & 33 & 10 & 5 & 15 \\
\hline RT21 & 1314 & 1469 & 2783 & 49 & 39 & 88 & 10 & 9 & 19 \\
\hline RT22 & 328 & 196 & 524 & 63 & 20 & 83 & 16 & 2 & 18 \\
\hline RT23 & 668 & 473 & 1141 & 54 & 21 & 75 & 11 & 7 & 18 \\
\hline WPRZ & 1689 & 2063 & 3752 & 356 & 317 & 673 & 125 & 120 & 245 \\
\hline Unique & 2204 & 2466 & 4670 & 1469 & 1358 & 2827 & 947 & 851 & 1798 \\
\hline
\end{tabular}


Table A.4: Percentage of measurements in each category. norm $/ n$ indicates event was part of GNS catalogue, det/d indicates the event was a matched filter detection and tot/t indicates a total. pick are the percentages of events successfully picked and sws are the percentages of high grade shear wave splitting measurements. Borehole stations are marked with $*$.

\begin{tabular}{|c|c|c|c|c|c|c|c|}
\hline Station & $\%$ det & $\%$ norm pick & $\%$ det pick & $\%$ pick & $\%$ norm sws & $\%$ det sws & $\%$ sws \\
\hline \hline ARAZ & 54 & 10 & 7 & 8 & 18 & 13 & 16 \\
\hline NS01 & 57 & 21 & 15 & 17 & 30 & 26 & 28 \\
\hline NS03 & 53 & 32 & 15 & 23 & 34 & 34 & 34 \\
\hline NS07 & 55 & 17 & 6 & 11 & 24 & 15 & 21 \\
\hline NS08 & 37 & 14 & 3 & 10 & 0 & 0 & 0 \\
\hline NS09 & 37 & 13 & 1 & 8 & 0 & 0 & 0 \\
\hline NS11 & 60 & 12 & 5 & 8 & 22 & 28 & 25 \\
\hline NS12* & 59 & 14 & 5 & 9 & 32 & 22 & 29 \\
\hline NS13* & 62 & 23 & 9 & 15 & 47 & 24 & 38 \\
\hline NS14 & 61 & 17 & 11 & 13 & 30 & 35 & 32 \\
\hline NS15 & 45 & 15 & 8 & 12 & 30 & 29 & 29 \\
\hline NS16 & 55 & 20 & 11 & 15 & 44 & 46 & 45 \\
\hline NS18 & 60 & 8 & 3 & 5 & 30 & 44 & 36 \\
\hline RT01 & 53 & 32 & 22 & 27 & 44 & 43 & 44 \\
\hline RT05 & 53 & 35 & 30 & 32 & 53 & 57 & 55 \\
\hline RT12 & 54 & 24 & 19 & 21 & 39 & 40 & 39 \\
\hline RT14 & 57 & 8 & 6 & 7 & 31 & 23 & 27 \\
\hline RT17 & 54 & 24 & 17 & 20 & 35 & 39 & 37 \\
\hline RT18 & 56 & 25 & 18 & 21 & 39 & 37 & 38 \\
\hline RT19 & 55 & 1 & 1 & 1 & 56 & 33 & 45 \\
\hline RT21 & 53 & 4 & 3 & 3 & 20 & 23 & 22 \\
\hline RT22 & 37 & 19 & 10 & 16 & 25 & 10 & 22 \\
\hline RT23 & 41 & 8 & 4 & 7 & 20 & 33 & 24 \\
\hline WPRZ & 55 & 21 & 15 & 18 & 35 & 38 & 36 \\
\hline Unique & 53 & 67 & 55 & 61 & 64 & 63 & 64 \\
\hline
\end{tabular}


Table A.5: Estimates of percent anisotropy $(k)$ and differential shear wave anisotropy (SWA) across full station-even paths (a) and station-event paths capped at $4.9 \mathrm{~km}(b)$.

\begin{tabular}{|c|c|c|c|c|}
\hline Station & $\mathrm{k}(\mathrm{a}) \%$ & SWA (a) $\%$ & $\mathrm{k}(\mathrm{b}) \%$ & $\mathrm{SWA}(\mathrm{b}) \%$ \\
\hline \hline ARAZ & 2.26 & 2.23 & 4.99 & 4.84 \\
\hline NS01 & 3.66 & 3.57 & 4.89 & 4.75 \\
\hline NS03 & 3.36 & 3.29 & 3.98 & 3.89 \\
\hline NS07 & 2.63 & 2.59 & 3.46 & 3.39 \\
\hline NS11 & 4.26 & 4.15 & 4.79 & 4.66 \\
\hline NS12 & 2.66 & 2.62 & 3.98 & 3.89 \\
\hline NS13 & 3.05 & 2.99 & 3.9 & 3.81 \\
\hline NS14 & 3.9 & 3.79 & 4.52 & 4.39 \\
\hline NS15 & 3.38 & 3.31 & 3.64 & 3.56 \\
\hline NS16 & 2.27 & 2.23 & 2.69 & 2.63 \\
\hline NS18 & 3.73 & 3.63 & 4.02 & 3.92 \\
\hline RT01 & 3.19 & 3.13 & 3.48 & 3.4 \\
\hline RT05 & 2.9 & 2.84 & 3.43 & 3.35 \\
\hline RT12 & 4.31 & 4.21 & 4.44 & 4.33 \\
\hline RT14 & 3.94 & 3.84 & 4.12 & 4.01 \\
\hline RT17 & 3.97 & 3.88 & 4.24 & 4.14 \\
\hline RT18 & 3.56 & 3.48 & 4 & 3.89 \\
\hline RT19 & 4.53 & 4.38 & 4.89 & 4.73 \\
\hline RT21 & 4.39 & 4.28 & 4.62 & 4.51 \\
\hline RT22 & 3.37 & 3.31 & 3.37 & 3.31 \\
\hline RT23 & 4.54 & 4.42 & 4.88 & 4.75 \\
\hline WPRZ & 2.42 & 2.38 & 4 & 3.9 \\
\hline
\end{tabular}


Appendix B

Fitted crack planes 
Table B.1: Table of fitted crack planes. Columns labelled with well name indicates if that particular group has no evidence that mean fast polarisation (of restricted measurements) is different from the mean $S_{H_{\max }}$ (p-value $\left.\geq 0.05\right)$. NA indicates that either there weren't enough restricted measurements to do the comparison or the restricted measurements do not have a dominant mean orientation (fail the Rayleigh test). Mean delay times of the (restricted) groups are also quoted ( $\delta t)$.

\begin{tabular}{|l|c|c|c|c|c|c|c|c|}
\hline & Strike $\left(^{\circ}\right)$ & Dip $\left(^{\circ}\right)$ & $\begin{array}{c}\text { Dip } \\
\text { Azimuth }\left(^{\circ}\right)\end{array}$ & $\delta t$ & RK18L2 & RK32 & RK30L1 & $\#$ \\
\hline \hline Group11 & 45 & 70 & 135 & 0.11 & Yes & Yes & No & 66 \\
\hline Group25 & 20 & 60 & 290 & 0.093 & No & No & No & 381 \\
\hline Group22 & 35 & 62.5 & 305 & 0.098 & No & No & No & 111 \\
\hline Group30 & 25 & 77.5 & 115 & 0.054 & No & Yes & Yes & 55 \\
\hline Group7 & 35 & 82.5 & 305 & 0.092 & No & Yes & No & 112 \\
\hline Group8 & -45 & 60 & 45 & 0.084 & NA & NA & NA & 69 \\
\hline Group14 & 0 & 70 & 90 & 0.081 & No & No & Yes & 55 \\
\hline Group38 & 35 & 87.5 & 305 & 0.096 & Yes & Yes & No & 98 \\
\hline Group28 & 80 & 62.5 & 350 & 0.08 & No & No & No & 123 \\
\hline Group18 & -45 & 65 & 45 & 0.092 & NA & NA & NA & 83 \\
\hline Group43 & 60 & 57.5 & 150 & 0.092 & NA & NA & NA & 81 \\
\hline Group26 & 75 & 40 & 165 & 0.092 & NA & NA & NA & 60 \\
\hline Group53 & 30 & 27.5 & 120 & 0.092 & NA & NA & NA & 53 \\
\hline Group34 & -20 & 65 & 70 & 0.103 & No & No & No & 179 \\
\hline Group37 & -65 & 22.5 & 25 & 0.092 & NA & NA & NA & 92 \\
\hline Group40 & -60 & 15 & 210 & 0.092 & NA & NA & NA & 108 \\
\hline Group3 & -40 & 65 & 50 & 0.142 & NA & NA & NA & 223 \\
\hline Group29 & 10 & 57.5 & 280 & 0.091 & NA & NA & NA & 129 \\
\hline Group31 & -60 & 52.5 & 30 & 0.092 & NA & NA & NA & 70 \\
\hline
\end{tabular}




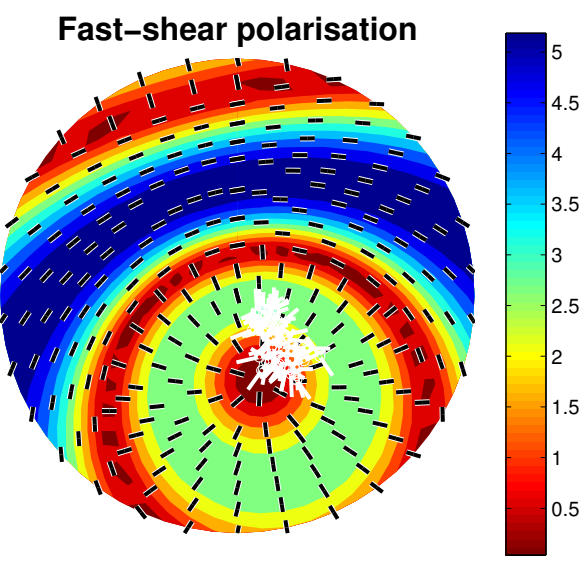

(a) Best fitting pole plot

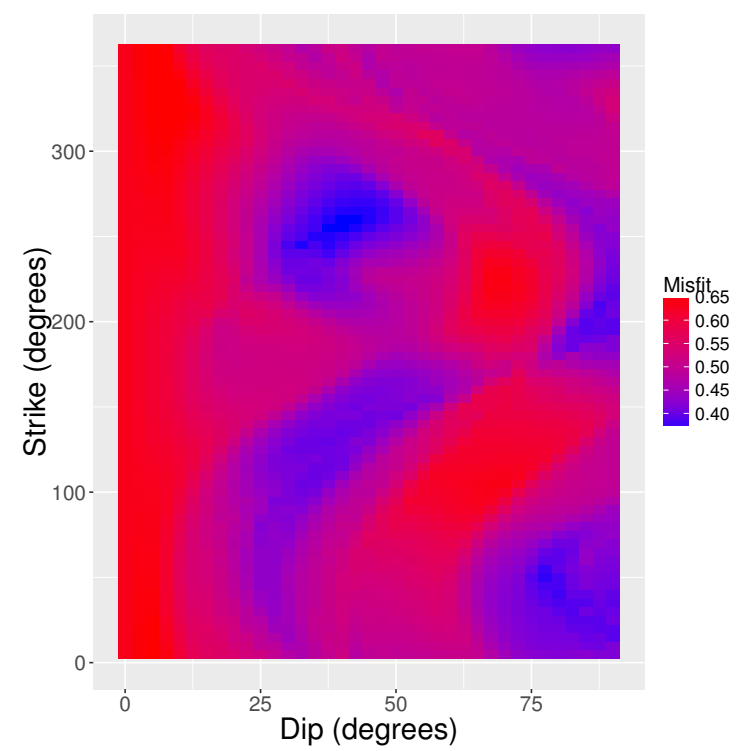

(b) Misfit grid

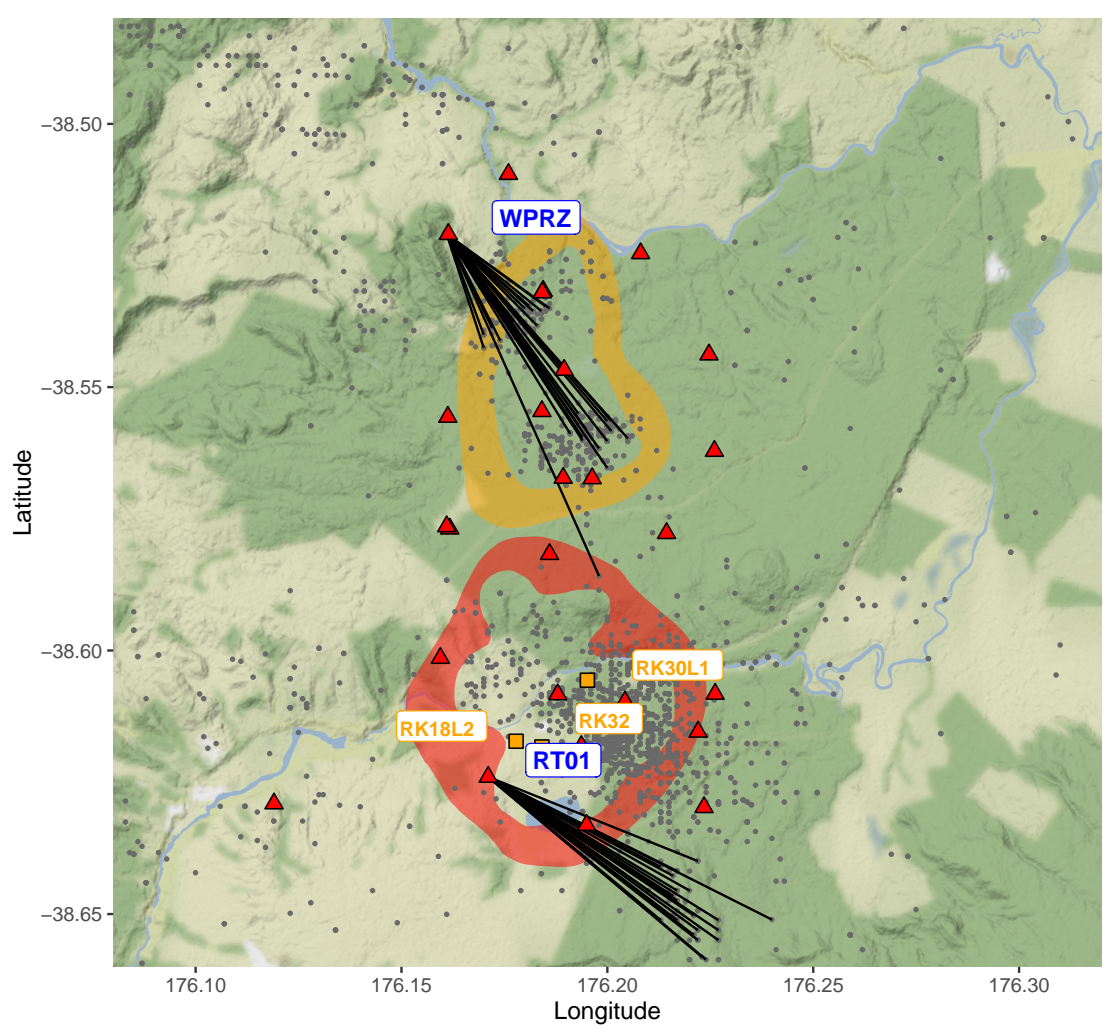

(c) Station-event paths for measurements in Group.

Figure B.1: Summary of Group 26. Best fitting cracks striking $75^{\circ}$. 


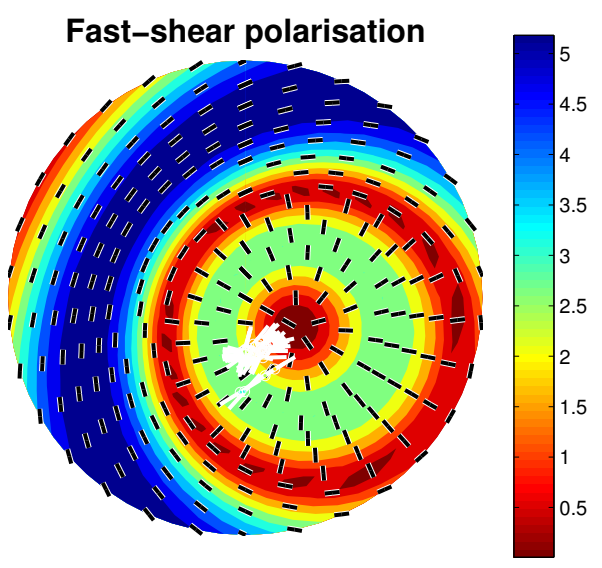

(a) Best fitting pole plot

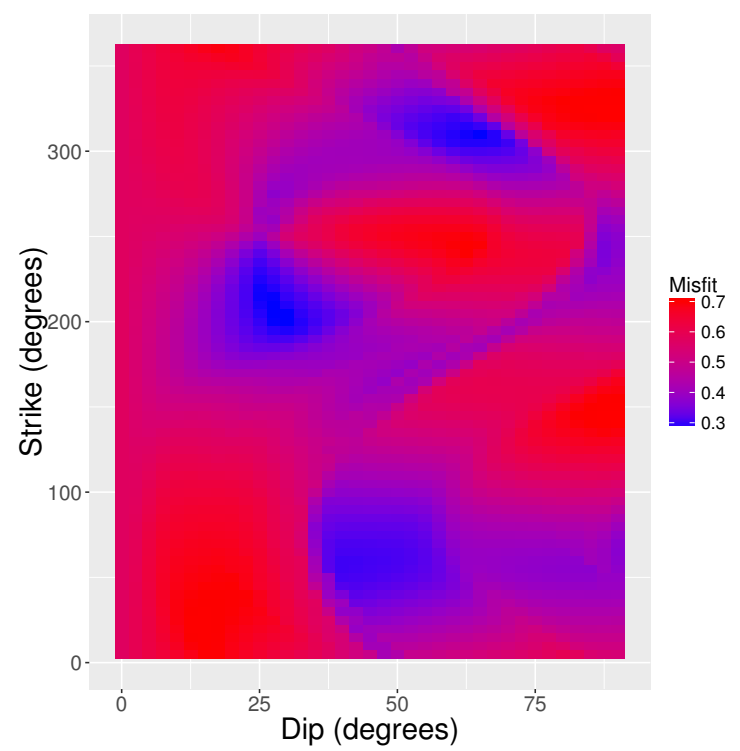

(b) Misfit grid

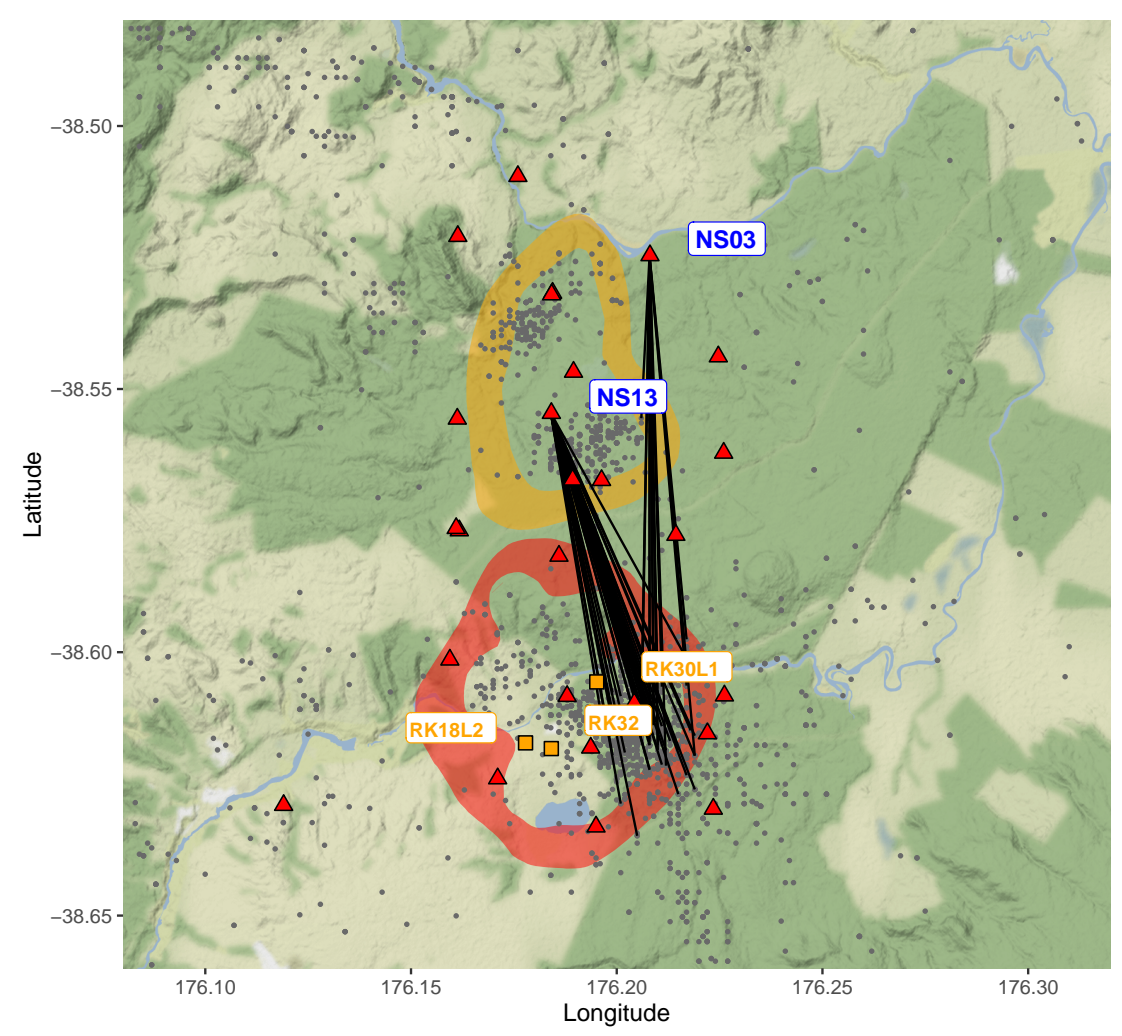

(c) Station-event paths for measurements in Group.

Figure B.2: Summary of Group 53. Best fitting cracks striking $30^{\circ}$. 


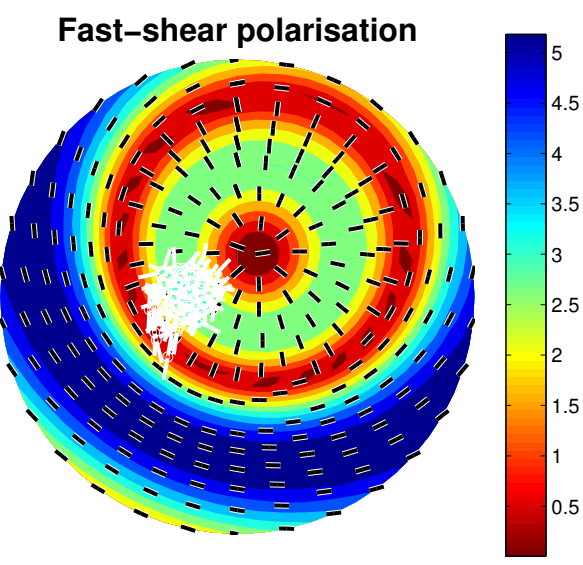

(a) Best fitting pole plot

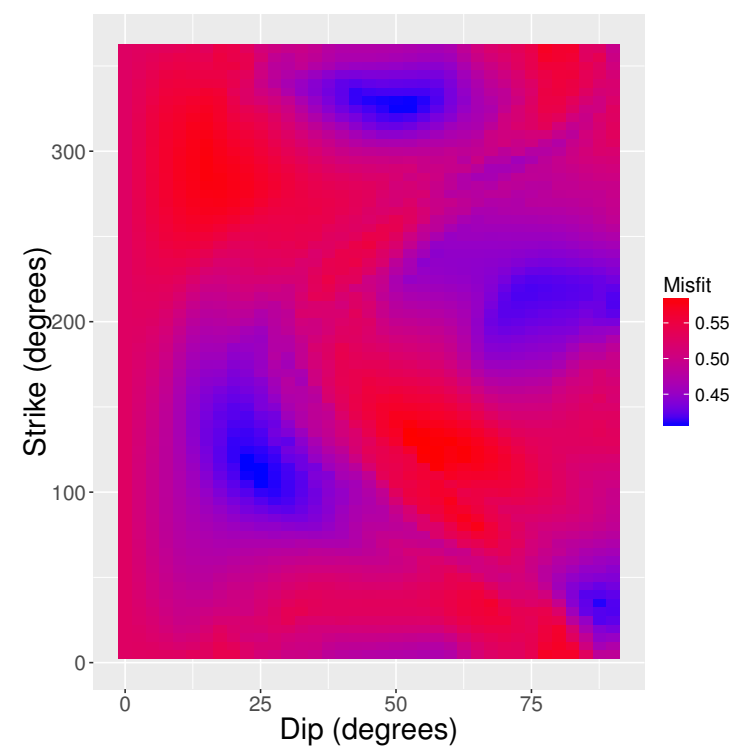

(b) Misfit grid

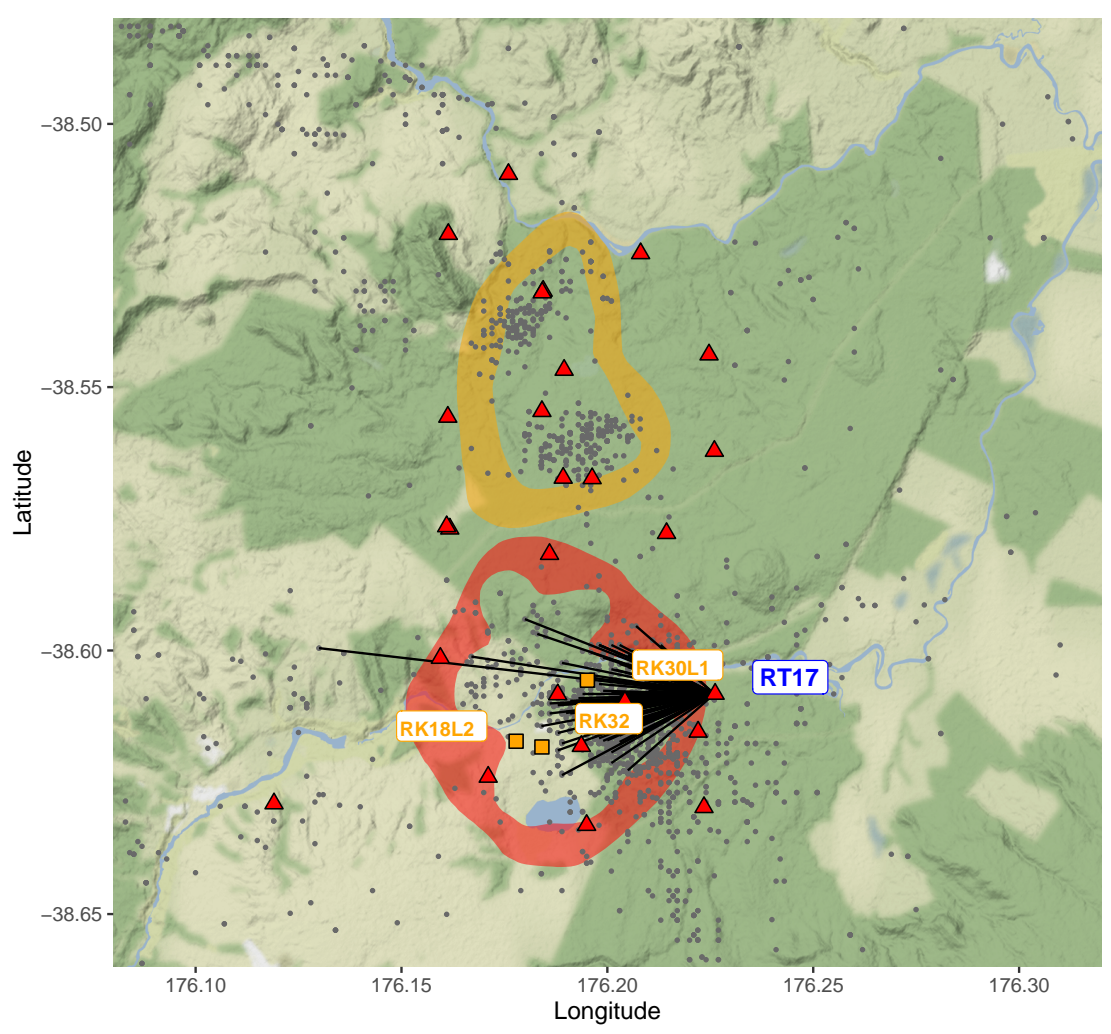

(c) Station-event paths for measurements in Group.

Figure B.3: Summary of Group 3\%. Best fitting cracks striking -65․ 


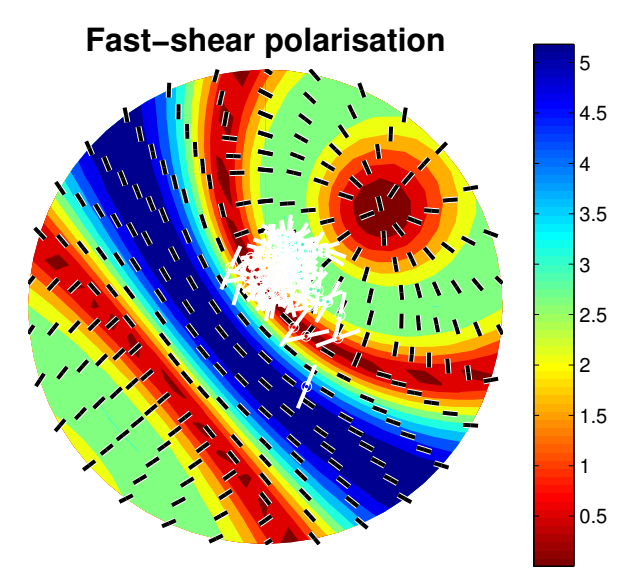

(a) Best fitting pole plot

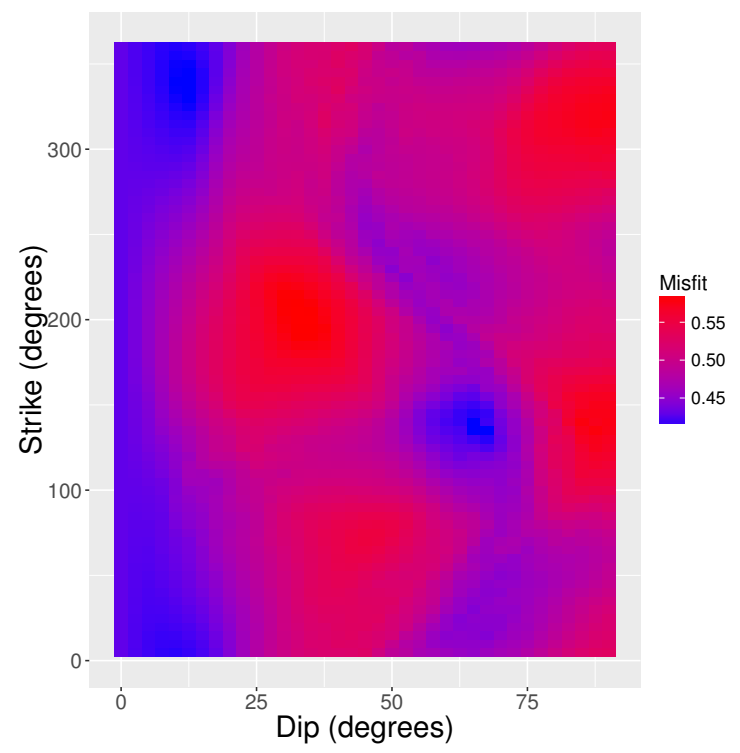

(b) Misfit grid

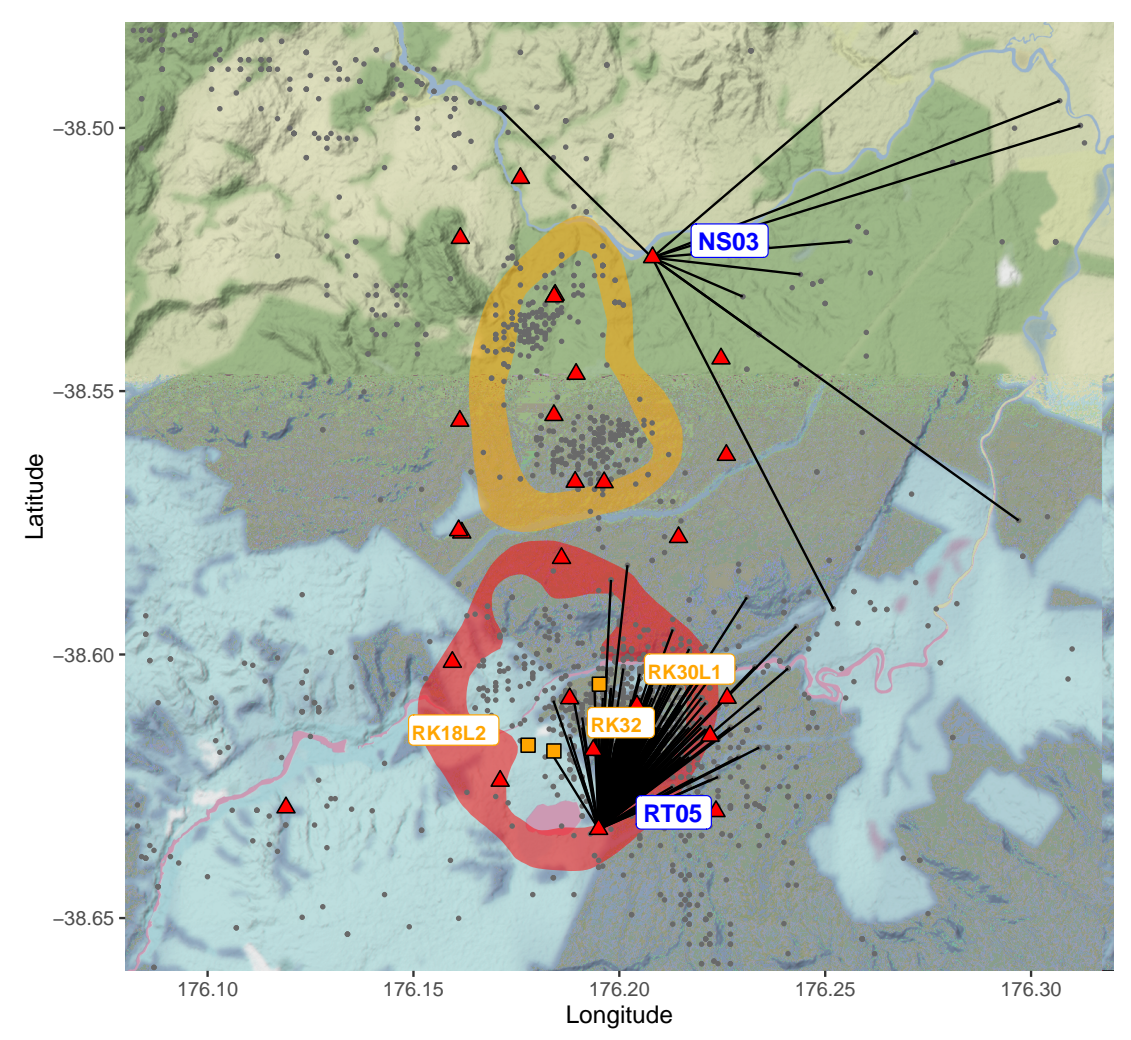

(c) Station-event paths for measurements in Group.

Figure B.4: Summary of Group 3. Best fitting cracks striking $-40^{\circ}$. 


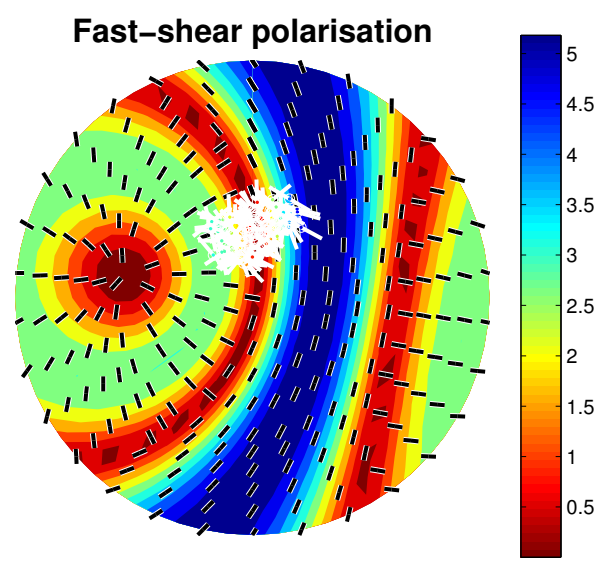

(a) Best fitting pole plot

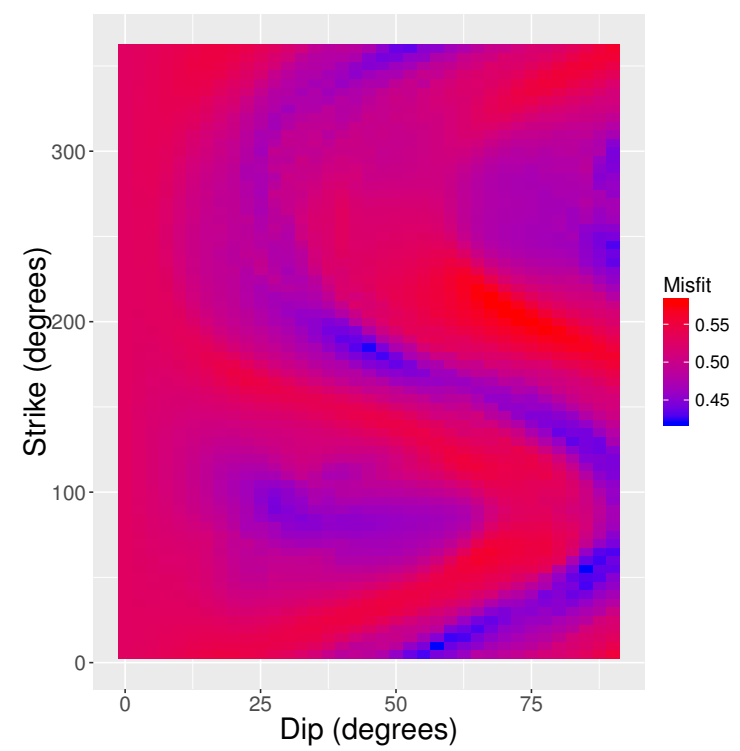

(b) Misfit grid

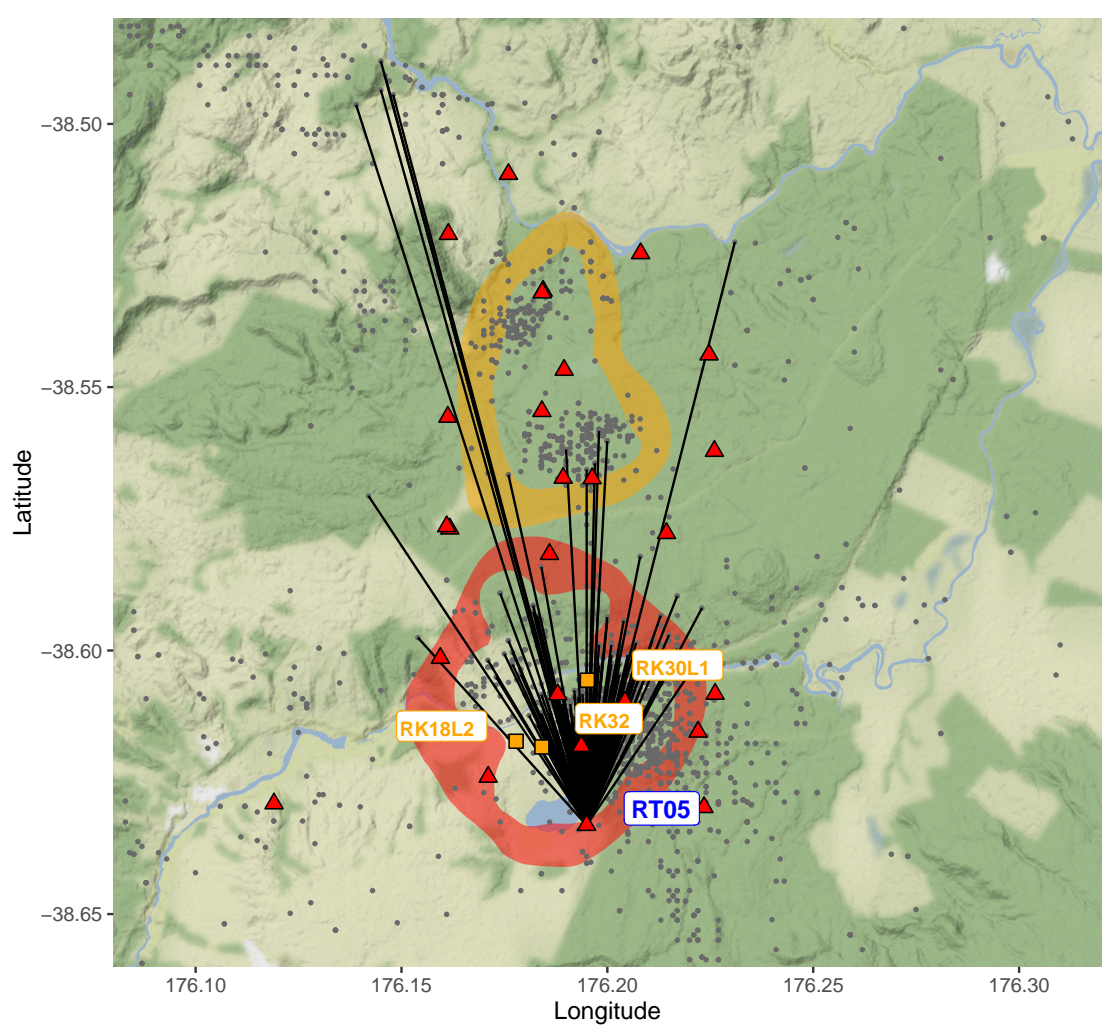

(c) Station-event paths for measurements in Group.

Figure B.5: Summary of Group 29. Best fitting cracks striking $10^{\circ}$. 


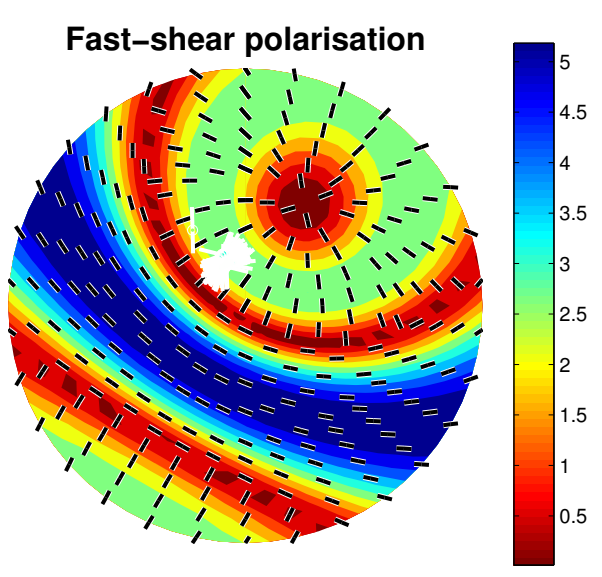

(a) Best fitting pole plot

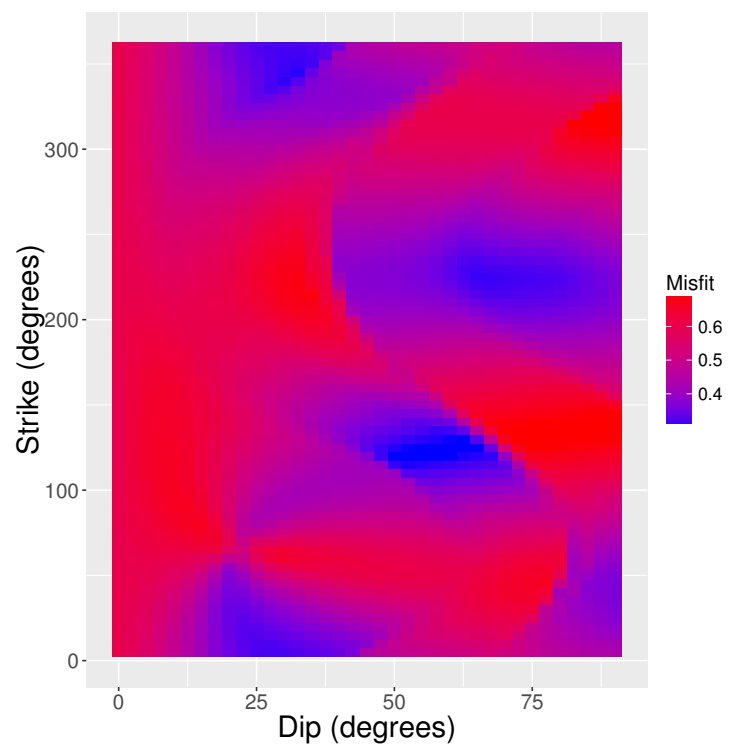

(b) Misfit grid

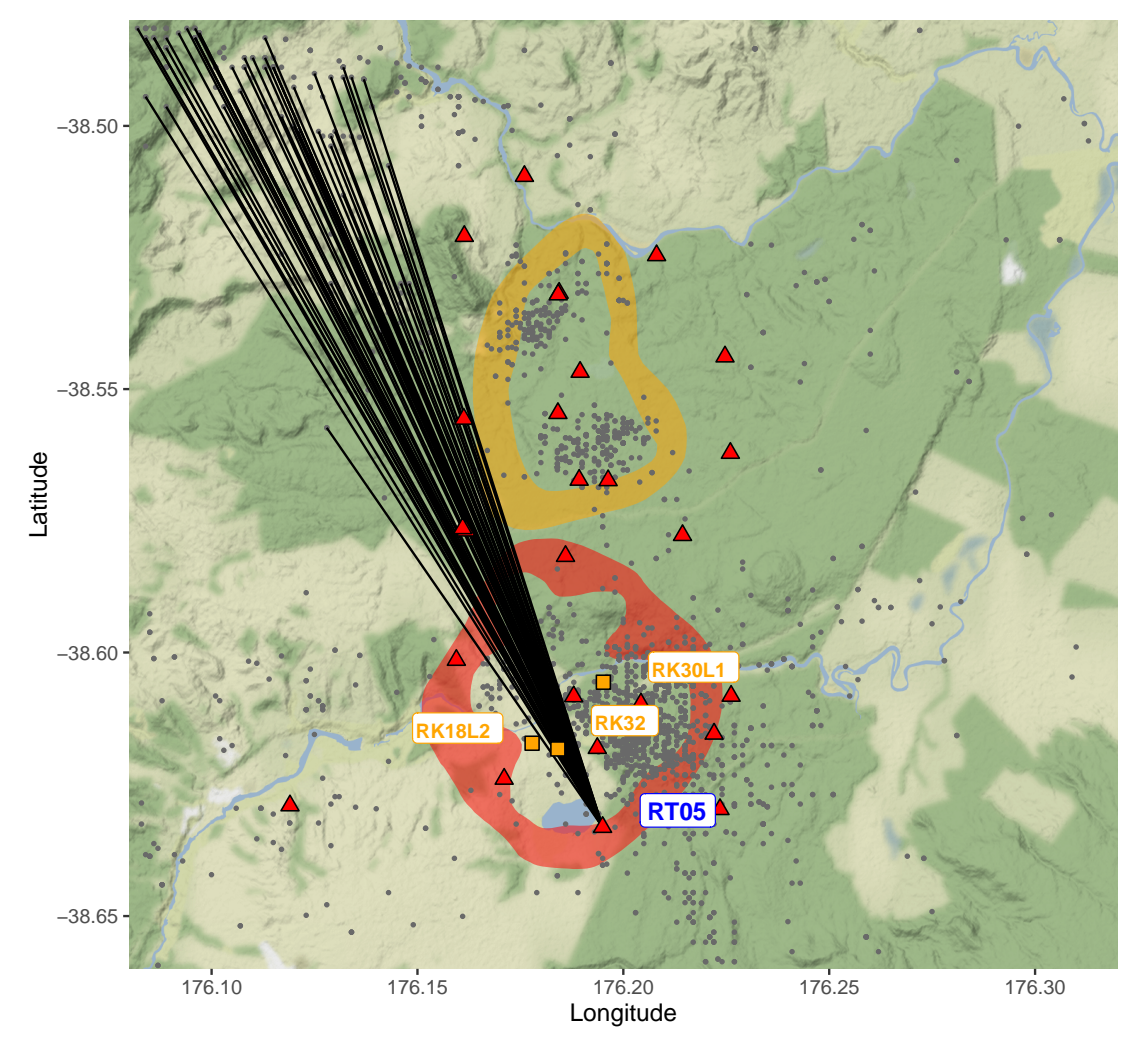

(c) Station-event paths for measurements in Group.

Figure B.6: Summary of Group 31. Best fitting cracks striking -60. 


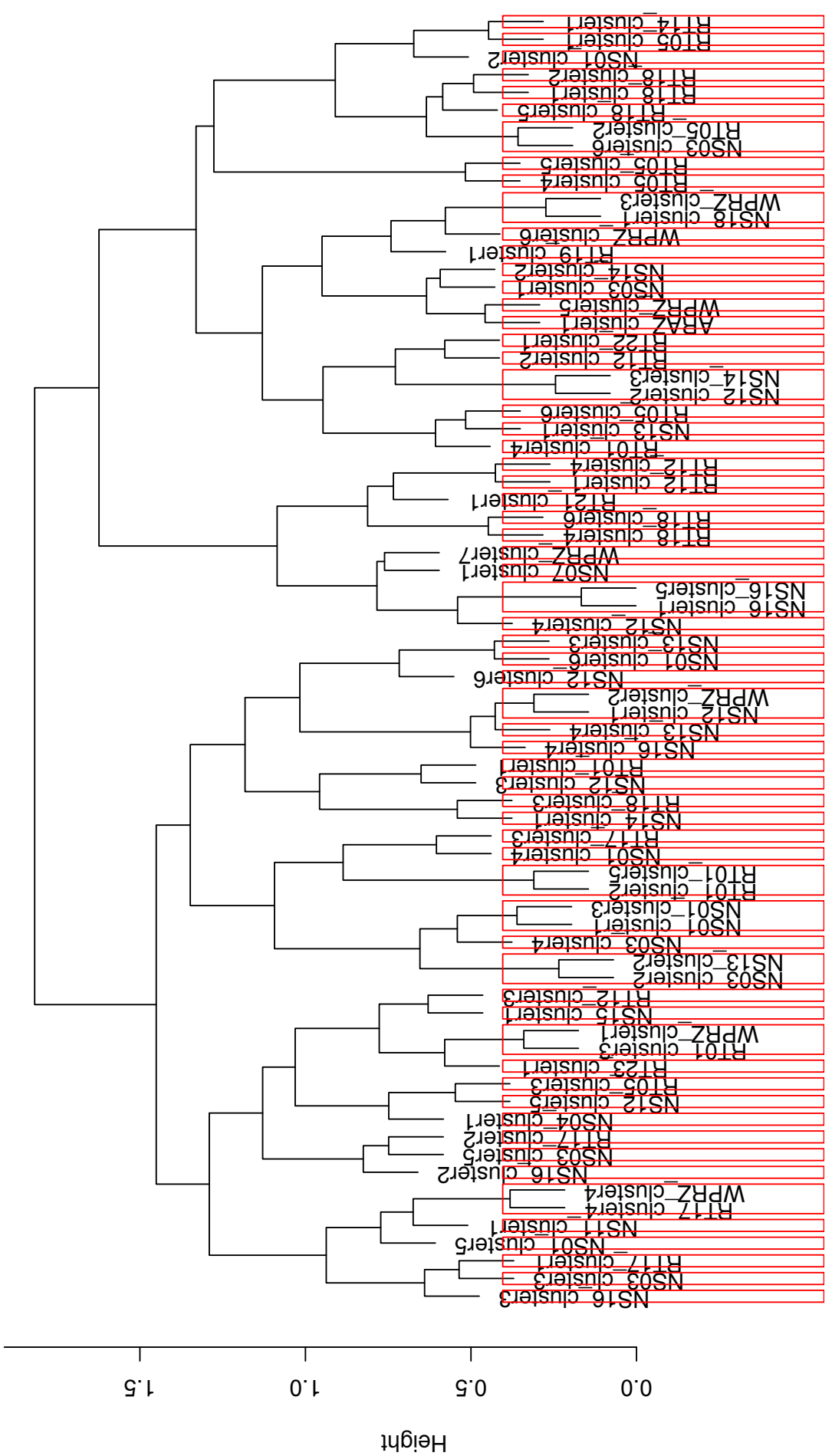

Figure B.7: Cluster dendrogram of correlated misfit grids for of station-event clusters. Clusters with 0.4 dissimilarity cut off are plotted in red. 
APPENDIX B. FITTED CRACK PLANES 


\section{Appendix C}

\section{Parameters}

\section{C.1 spickerC}

Table C.1: Table of spickerC shear wave picking parameters used in this thesis. After Castellazzi et al. (2015) supporting information. * marks parameters which have changed from Castellazzi et al. (2015) supporting information (Ruapehu).

\begin{tabular}{|l|l|l|}
\hline Parameter & Value & Description \\
\hline \hline Despike* $^{*}$ & 1 & Despike option enabled \\
\hline Automatic final pick class0 definition & $\begin{array}{l}\text { Maximum final error al- } \\
\text { lowed }\end{array}$ \\
\hline Class0 max error* & 0.3 & $\begin{array}{l}\text { Maximum final SNR al- } \\
\text { lowed }\end{array}$ \\
\hline Class0 max SNR & 3 & \multicolumn{2}{|l|}{} \\
\hline Coarse window parameters & 1.2 & Minimum $v_{P}$ allowed \\
\hline kpmin $*$ & 2.2 & $v_{S}$ allowed \\
\hline kpmax* & & \\
\hline STA/LTA and Polarisation detector parameters \\
\hline
\end{tabular}




\begin{tabular}{|c|c|c|}
\hline$t M H A$ & \multicolumn{2}{|c|}{$\begin{array}{l}\text { Time of maximum amplitude on the horizon- } \\
\text { tal component (coarse window) }\end{array}$} \\
\hline S2tMHA (sec) & 3 & $\begin{array}{l}\text { Maximum time expected for } \\
\text { the } \mathrm{S} \text { arrival before tMHA }\end{array}$ \\
\hline $\operatorname{sta}^{*}(\mathrm{sec})$ & 0.15 & STA window length \\
\hline$l t a^{*}(\mathrm{sec})$ & 1.5 & LTA window length \\
\hline tup $^{*}(\mathrm{sec})$ & 0.1 & $\begin{array}{l}\text { Minimum time the charac- } \\
\text { teristic function must stay } \\
\text { above the maximum value }\end{array}$ \\
\hline$t d w^{*}(\mathrm{sec})$ & 0.05 & $\begin{array}{l}\text { Maximum time the charac- } \\
\text { teristic function can drop } \\
\text { below the threshold }\end{array}$ \\
\hline \multicolumn{3}{|l|}{ AR-AIC processing parameters } \\
\hline Searching window* (sec) & $1.5(0.75+0.75)$ & $\begin{array}{l}\text { Searching window around } \mathrm{S} \\
\text { arrival from other methods }\end{array}$ \\
\hline Noise model window* (sec) & 1.1 & Noise model window length \\
\hline Signal model window* (sec) & 1.1 & Signal model window length \\
\hline Order noise model* & 15 & $\begin{array}{l}\text { Maximum order of the AR } \\
\text { forward model }\end{array}$ \\
\hline Order signal model* & 16 & $\begin{array}{l}\text { Maximum order of the AR } \\
\text { backward model }\end{array}$ \\
\hline \multicolumn{3}{|c|}{ Converted $\mathrm{P}$ detection parameters } \\
\hline checkwlen (sec) & 0.3 & $\begin{array}{l}\text { Window around STA/LTA } \\
\text { pick for checking for con- } \\
\text { verted P }\end{array}$ \\
\hline
\end{tabular}




\begin{tabular}{|l|l|l|}
\hline H2Zratio & 1 & $\begin{array}{l}\text { Ratio between horizontal } \\
\text { and vertical component } \\
\text { maximum amplitude inside } \\
\text { checkwlen window }\end{array}$ \\
\hline
\end{tabular}

\section{C.2 MFAST}

Table C.2: Table of MFAST shear wave splitting parameters used in this study (based on Wessel et al. (2016)).

\begin{tabular}{|c|c|c|c|}
\hline Parameter name & Description & $\begin{array}{l}\text { Expected } \\
\text { Sensitivity }\end{array}$ & Value \\
\hline$h i$ and $l o$ filters & See Table 3.2 & High & Table 3.2 \\
\hline$t \_w i n \_s n r$ & $\begin{array}{l}\text { Window for SNR cal- } \\
\text { culation }\end{array}$ & Low & $0.75 \mathrm{~s}$ \\
\hline$t_{-} e r r$ & $\begin{array}{l}\text { Accounts for error in } \\
\text { S-wave picks }\end{array}$ & Low & $0.02 \mathrm{~s}$ \\
\hline SNRmax & $\begin{array}{l}\text { Minimum SNR al- } \\
\text { lowed }\end{array}$ & Medium & 3 \\
\hline tlagmax & $\begin{array}{l}\text { Maximum time delay } \\
\text { allowed }\end{array}$ & High & $0.4 \mathrm{~s}$ \\
\hline tlagscale & $\begin{array}{l}\text { Scale on plots of error } \\
\text { contours }\end{array}$ & & $0.4 \mathrm{~s}($ tlagmax $)$ \\
\hline dtlag_max & $\begin{array}{l}\text { Maximum tlag error } \\
\text { allowed }\end{array}$ & High & $\begin{array}{c}0.1 \mathrm{~s} \\
(\operatorname{tlagmax} / 4)\end{array}$ \\
\hline$t_{-} w i n_{-} f r e q$ & $\begin{array}{l}\text { used to calculate dom- } \\
\text { inant frequency }(f d)\end{array}$ & Low & $0.75 \mathrm{~s}$ \\
\hline
\end{tabular}




\begin{tabular}{|c|c|c|c|}
\hline Tmid & Dominant period & & $1 / f d$ \\
\hline fdmin, fdmax & $\begin{array}{l}\text { Minimum and maxi- } \\
\text { mum allowed values of } \\
f d\end{array}$ & High & $\begin{array}{l}1 / t_{-} w i n \_s n r \text { to } \\
\text { samplerate } / 10\end{array}$ \\
\hline$w 3$ & $\begin{array}{l}\text { Desired last end win- } \\
\text { dow time }\end{array}$ & & $\operatorname{Tmid} * 1.2+0.15$ \\
\hline$w 4$ & $\begin{array}{l}\text { Desired last end win- } \\
\text { dow time }\end{array}$ & & $\operatorname{Tmid} * 2.5+0.15$ \\
\hline$d t_{b e g}$ & & Low & $\begin{array}{l}0.2 \mathrm{~s} \text { (decreased } \\
\text { to } 0.1 \text { or }((S- \\
P) / 2) / N b e g \text { if } \\
\text { S-P time } \\
\text { difference is too } \\
\text { small) }\end{array}$ \\
\hline$d t_{\text {end }}$ & & High & $\begin{array}{c}0.08 \text { s but } \\
\text { allowed to vary } \\
\text { so Nend is } \\
\text { between is } \\
\text { between } 15 \text { and } \\
\text { 2. Determined } \\
\text { from } w 3 \text { and } \\
w 4 .\end{array}$ \\
\hline$N_{w b e g}$ & $\begin{array}{l}\text { Number of start times } \\
\text { tested }\end{array}$ & Low & 5 \\
\hline
\end{tabular}




\begin{tabular}{|c|c|c|c|}
\hline$N_{\text {wend }}$ & $\begin{array}{l}\text { Number of end times } \\
\text { tested }\end{array}$ & & $\begin{array}{l}(w 4-w 3) / d t_{\text {end }} \\
\text { rounded to the } \\
\text { nearest integer }\end{array}$ \\
\hline Nbeg & $\begin{array}{l}\text { Number of start win- } \\
\text { dow times }\end{array}$ & low & 5 \\
\hline Nend & $\begin{array}{l}\text { Number of end win- } \\
\text { dow times }\end{array}$ & low & $\begin{array}{c}\text { Between } 2 \text { and } \\
\text { 15. Based on } \\
d t_{\text {end }}\end{array}$ \\
\hline Tbeg0 & $\begin{array}{l}\text { First time to start } \\
\text { window }\end{array}$ & & $\begin{array}{l}0.3 \mathrm{~s} \text { (decreased } \\
0.1 \text { or }((S- \\
P) / 2) /(N b e g+ \\
1) \text { if S-P time } \\
\text { difference is too } \\
\text { small) }\end{array}$ \\
\hline Tbeg1 & $\begin{array}{l}\text { Last time to start win- } \\
\text { dow }\end{array}$ & & $\begin{array}{c}S_{\text {arrival }}- \\
(\text { Tbeg } 0+ \\
\left.\left(\text { Nbeg } * d t_{\text {beg }}\right)\right)\end{array}$ \\
\hline Tend0 & $\begin{array}{l}\text { First time to end win- } \\
\text { dow }\end{array}$ & & $\begin{array}{c}S_{\text {arrival }}+ \\
\text { Tmid } / 1.2+0.15\end{array}$ \\
\hline Tend1 & $\begin{array}{l}\text { Last time to end win- } \\
\text { dow }\end{array}$ & & $\begin{array}{c}S_{\text {arrival }}+ \\
\text { Tend } 0+N \text { end } * \\
d t_{\text {end }}\end{array}$ \\
\hline Ncmin & $\begin{array}{l}\text { Minimum number of } \\
\text { points in an accept- } \\
\text { able cluster }\end{array}$ & & 5 \\
\hline
\end{tabular}


APPENDIX C. PARAMETERS

\begin{tabular}{|l|l|l|}
\hline Mmax & $\begin{array}{l}\text { Maximum number of } \\
\text { clusters }\end{array}$ & 15 \\
\hline
\end{tabular}




\section{Appendix D}

\section{MSAT functions}

\section{D.1 MS_hudson_cracks}

To fit the data we require an elastic model for fluid-filled cracks. The fluid-filled crack model we use is that of (Hudson 1980; 1981) which is used frequently to describe the behaviour of seismic waves in a crack solid (e.g. Peacock et al. 1988; Elkibbi et al. 2005; Savage et al. 2010b).

In the MSAT package the MS_hudson_cracks function produces an elasticity matrix for a given matrix and crack inclusion material. We use water as the inclusion material (for fluid-filled cracks).

Here we describe the process MS_hudson_cracks which draws largely from Crampin (1984). MS_hudson_cracks takes the inputs for body wave velocities $\left(v_{p}, v_{s}\right)$ for both the crack and matrix. Along with density $(\rho)$, these are used to calculate Lamé's first and second parameters $(\lambda, \mu)$ and bulk modulus $(\kappa)$.

$$
\begin{gathered}
\mu=v_{s}^{2} * \rho \\
\mu_{\text {crack }}=v_{s_{\text {crack }}}^{2} * \rho_{\text {crack }} \\
\lambda=v_{p}^{2} * \rho-2 \mu
\end{gathered}
$$




$$
\begin{gathered}
\lambda_{\text {cracK }}=v_{p_{\text {crack }}}^{2} * \rho_{\text {crack }}-2 \mu_{\text {crack }} \\
\kappa=\lambda_{\text {crack }}+\frac{2}{3} \mu_{\text {crack }}
\end{gathered}
$$

We can then begin calculating elements of the elasticity matrix using Hudson (1981)'s formulations:

$$
\begin{gathered}
U_{1}^{1}=(4 / 3)[(\lambda+2 \mu) /(\lambda+\mu)] /(1+K) \\
U_{3}^{3}=(16 / 3)[(\lambda+2 \mu) /(3 \lambda+4 \mu)] /(1+M)
\end{gathered}
$$

Where

$$
\begin{gathered}
K=\left[\left(\kappa+\frac{4}{3} \mu_{\text {crack }}\right) /(\pi * d * \mu)\right][(\lambda+2 \mu) /(\lambda+\mu)] \\
M=\left[4 \mu_{\text {crack }} /(\pi * d * \mu)\right][(\lambda+2 \mu) /(3 \lambda+4 \mu)]
\end{gathered}
$$

and $d$ is aspect ratio of the cracks. The first order effects of the cracks is thus written as:

$$
C_{1}=\left[\begin{array}{cccccc}
U_{1}^{1}(\lambda+2 \mu)^{2} & U_{1}^{1}(\lambda+2 \mu) \lambda & U_{1}^{1}(\lambda+2 \mu) \lambda & 0 & 0 & 0 \\
U_{1}^{1}(\lambda+2 \mu) \lambda & U_{1}^{1} \lambda^{2} & U_{1}^{1} \lambda^{2} & 0 & 0 & 0 \\
U_{1}^{1}(\lambda+2 \mu) \lambda & U_{1}^{1} \lambda^{2} & U_{1}^{1} \lambda^{2} & 0 & 0 & 0 \\
0 & 0 & 0 & 0 & 0 & 0 \\
0 & 0 & 0 & 0 & U_{3}^{3} \mu^{2} & 0 \\
0 & 0 & 0 & 0 & 0 & U_{3}^{3} \mu^{2}
\end{array}\right] \frac{-\epsilon}{\mu}
$$

and the second order effects (Hudson 1980):

$$
C_{2}=\left[\begin{array}{cccccc}
\left(U_{1}^{1}\right)^{2}(\lambda+2 \mu) q & \left(U_{1}^{1}\right)^{2} \lambda q & \left(U_{1}^{1}\right)^{2} \lambda q & 0 & 0 & 0 \\
\left(U_{1}^{1}\right)^{2} \lambda q & \left(U_{1}^{1}\right)^{2} \lambda^{2} q /(\lambda+2 \mu) & \left(U_{1}^{1}\right)^{2} \lambda^{2} q /(\lambda+2 \mu) & 0 & 0 & 0 \\
\left(U_{1}^{1}\right)^{2} \lambda q & \left(U_{1}^{1}\right)^{2} \lambda^{2} q /(\lambda+2 \mu) & \left(U_{1}^{1}\right)^{2} \lambda^{2} q /(\lambda+2 \mu) & 0 & 0 & 0 \\
0 & 0 & 0 & 0 & 0 & 0 \\
0 & 0 & 0 & 0 & \left(U_{3}^{3}\right)^{2} X & 0 \\
0 & 0 & 0 & 0 & 0 & \left(U_{3}^{3}\right)^{2} X
\end{array}\right] \frac{\epsilon^{2}}{15}
$$

Where $\epsilon$ is crack density defined as $\epsilon=\frac{N a^{3}}{v}$ where $N$ is the number of "penny" shaped cracks of radius $a$ in a volume $v$ (i.e. Budiansky and O'connell 1976).

and:

$$
q=15(\lambda / \mu)^{2}+28(\lambda / \mu)+28
$$




$$
X=2 \mu(3 \lambda+8 \mu) /(\lambda+2 \mu)
$$

Host rock matrix:

$$
C_{h}=\left[\begin{array}{cccccc}
v_{P}^{2} \rho & v_{P}^{2} \rho-2 v_{S}^{2} \rho & v_{P}^{2} \rho-2 v_{S}^{2} \rho & 0 & 0 & 0 \\
v_{P}^{2} \rho-2 v_{S}^{2} \rho & v_{P}^{2} \rho & v_{P}^{2} \rho-2 v_{S}^{2} \rho & 0 & 0 & 0 \\
v_{P}^{2} \rho-2 v_{S}^{2} \rho & v_{P}^{2} \rho-2 v_{S}^{2} \rho & v_{P}^{2} \rho & 0 & 0 & 0 \\
0 & 0 & 0 & v_{S}^{2} \rho & 0 & 0 \\
0 & 0 & 0 & 0 & v_{S}^{2} \rho & 0 \\
0 & 0 & 0 & 0 & 0 & v_{S}^{2} \rho
\end{array}\right]
$$

Thus, the effective elastic tensor of the fluid-filled crack medium is:

$$
C_{\text {eff }}=C_{h}+C_{1}+C_{2}
$$

\section{D.2 MS_rot3}

To adjust the strike and dip of the cracks given by the elasticity matrix (from MS_hudson_cracks) for the fitting process we must be able to rotate the elastic tensor. This is achieved using the MS_rot3 function to rotate around -dip ( $\beta$ ) and strike $(\gamma)$ using the method in Chapter 3 of Bower (2009).

Firstly the required rotation matrices are calculated. These are the usual matrices for rotating through some angle in 3 dimensions (e.g. Anton and Busby 2002 Chapter 6). It should be noted that the form here is for rotating through a negative angle as opposed to the usual convention (i.e. if we are rotating by $-\theta$ then $\cos (-\theta)=\cos (\theta)$ and $\sin (-\theta)=-\sin (\theta)$ ). We do not rotate around the $x_{1}$ direction (yaw).

$$
R_{\text {dip }}=\left[\begin{array}{ccc}
\cos (\beta) & 0 & -\sin (\beta) \\
0 & 1 & 0 \\
\sin (\beta) & 0 & \cos (\beta)
\end{array}\right]
$$




$$
R_{\text {strike }}=\left[\begin{array}{ccc}
\cos (\gamma) & \sin (\gamma) & 0 \\
-\sin (\gamma) & \cos (\gamma) & 0 \\
0 & 0 & 1
\end{array}\right]
$$

As with any rotation matrix these can be combined by multiplying them together

$$
\mathbf{R}=R_{\text {dip }} * R_{\text {strike }}
$$

Per Bower (2009), the basis change matrix for the elasticity matrix is

$$
\mathbf{K}=\left[\begin{array}{cc}
\mathbf{K}^{(1)} & 2 \mathbf{K}^{(2)} \\
\mathbf{K}^{(3)} & \mathbf{K}^{(4)}
\end{array}\right]
$$

Where (for $i, j$ in $1,2,3$ )

$$
\begin{gathered}
\mathbf{K}_{i j}^{(1)}=\mathbf{R}_{i j}^{2} \\
\mathbf{K}_{i j}^{(2)}=\mathbf{R}_{i \bmod (j+1,3)} \mathbf{R}_{i \bmod (j+2,3)} \\
\mathbf{K}_{i j}^{(3)}=\mathbf{R}_{i \bmod (i+1,3) j} \mathbf{R}_{\bmod (i+2,3) j} \\
\mathbf{K}_{i j}^{(4)}=\mathbf{R}_{i \bmod (i+1,3) \bmod (j+1,3)} \mathbf{R}_{i \bmod (i+2,3) \bmod (j+2,3)} \\
+\mathbf{R}_{i \bmod (i+1,3) \bmod (j+2,3)} \mathbf{R}_{i \bmod (i+2,3) \bmod (j+1,3)}
\end{gathered}
$$

mod () is the modulo function which satisfies

$$
\bmod (k, 3)= \begin{cases}k & k \leq 3 \\ k-3 & k>3\end{cases}
$$

e.g. evaluating $\bmod (i+1,3)$ for $i=1 \bmod (i+1,3)=\bmod (2,3)$ thus $k=2$ and $k \leq 3$ so $\bmod (i+1,3)=2$.

For some elasticity matrix $\mathbf{C}$ the rotated matrix is given by

$$
\mathbf{C}_{r o t}=\mathbf{K C K}^{T}
$$




\section{D.3 MS_phasevels}

MS_phase_levels calculates the expected velocities for a particular propagation angle and elasticity matrix by determining the Christoffel matrix and its corresponding eigenvalues and vectors. The eigenvectors and values giving the velocities and polarisations of the constituent waves travelling through the medium. We have an example of this calculation in Section 2.2.2. 


\section{Appendix E}

\section{R tools for MFAST}

\section{E.1 Background}

R-tools-for-MFAST facilitates fast and automated dissemination and statistical examination of shear wave splitting measurements made by MFAST.

$\mathrm{R}$ is a free software environment designed for statistical computing ( $\mathrm{R}$ Core Team 2013). Due to its wide usage (especially among the statistics community), there many packages are available for various applications most of which are statistical in nature. For this project we take advantage of several of these (referenced in the body of this thesis).

To analyse the shear wave splitting data we have almost exclusively used R. To achieve this we have written a number of functions and scripts designed specifically to read and analyse the data. These are available on github (http://github.com/ shearwavesplitter/R-tools-for-MFAST) and are documented here. The eventual aim is to be build these functions into a package written to a standard high enough to submit to CRAN (The Comprehensive R Archive Network).

All functions and scripts are written in $\mathrm{R}$ version 3.1.2. Compatibility depends mostly on packages being available for a particular release. 


\section{E.1.1 Required packages}

These packages are available on the CRAN website (https://cran.r-project.org) or can be directly downloaded from within $\mathrm{R}$.

- circular - For dealing with circular quantities (in our case axial polarisations) (Agostinelli and Lund 2013). Pewsey et al. (2013) provides a good overview of using circular statistics in $\mathrm{R}$ using this package.

- movMF - (Hornik and Grün 2014) See Section 3.7.1.

- RColorBrewer - Colour palettes for plots (Neuwirth 2014)

- fields Curve, surface and function fitting for spatial data (Nychka et al. 2015)

- ggplot2 - For producing plots (Wickham 2009). Used for plots in this thesis

- ggmap - For producing maps (Kahle and Wickham 2013). Used for maps in this thesis

\section{E.2 Functions}

\section{E.2.1 sm.createTESSA}

\section{Description}

Creates a TESSA input file

\section{Usage}

sm.createTESSA(summfile, name="out.summ", path=" $"$ ") 


\author{
Arguments \\ summfile \\ name Name of the output file \\ path Location to save output
}

\title{
Details
}

sm.createTESSA creates an input file for the TESSA codes using a summfile that has been read (and graded etc.) within R.

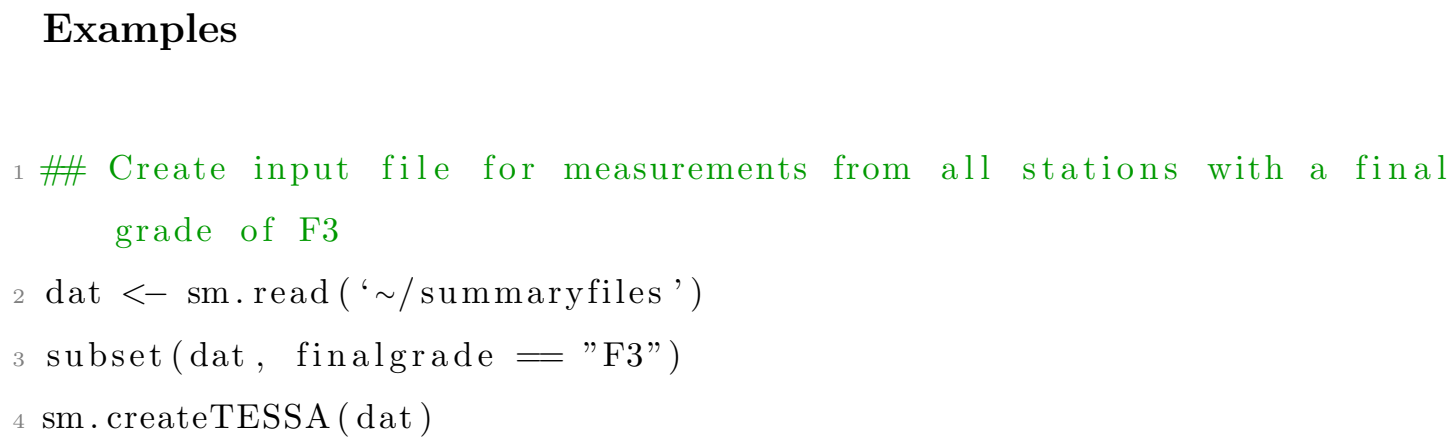

\section{E.2.2 sm.getevents}

\section{Description}

Return specific measurements from a dataframe

\author{
Usage \\ sm.getevents (summ, events, station $=$ NULL )
}




\section{Arguments}

summ .summ dataframe (output of sm.read)

events a vector containing event cuspid

station Station, NULL for all stations

\section{Details}

sm.getevents returns a dataframe of measurements with cuspids listed in the events vector.

\section{Examples}

1 \#\# Return measurements for events with cuspids stored in text file

2 dat $<-$ sm.read ( ' / summaryfiles')

$3 \operatorname{cs}<-$ read.table (' $\sim / R_{-}$MFAST/cluster 1 ') \#Read a table of cuspids

4 sm. getvents (dat, cs $\$ V 1$, station="WPRZ")

\section{E.2.3 sm.getevents}

\section{Description}

Return specific events from dataframe

\section{Usage}

sm.getevents(summ, events, station=NULL )

\section{Arguments}

summ .summ dataframe (output of sm.read)

events a vector containing event cuspid

station Station, NULL for all stations 


\section{Details}

sm.getevents returns a dataframe of measurements with cuspids listed in the events vector.

\section{Examples}

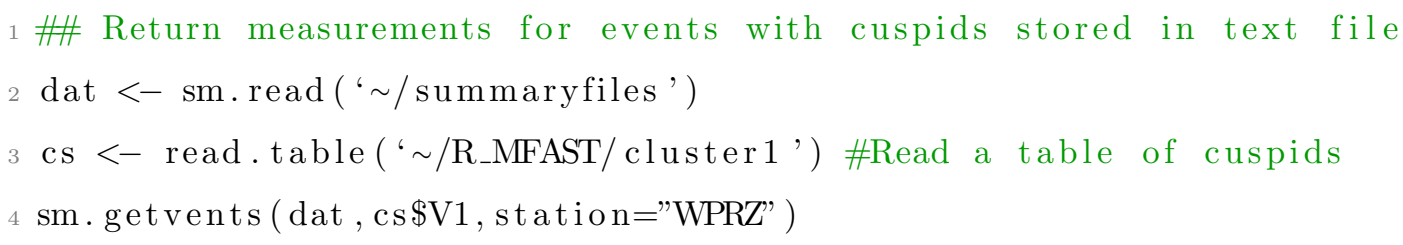

\section{E.2.4 sm.pathclus}

\section{Description}

Clustering of station to event paths

\section{Usage}

sm. pathclus (data, hvec $=$ NULL, $k \max =7$, path $="$ " ", plotextra $=$ TRUE, $\operatorname{rot}=180$ )

\section{Arguments}

$\begin{array}{ll}\text { data } & \text {.summ dataframe (output of sm.read) } \\ \text { hvec } & \text { Vector of station elevations (in order of unique(data } \$ \text { stat)) } \\ \text { kmax } & \text { Maximum number of clusters for each station } \\ \text { path } & \text { Path to save clusters and cluster figures } \\ \text { plotextra } & \text { Include 2D and 3D plots (TRUE/FALSE) } \\ \text { rot } & \text { Rotation of 3D plot of piece points }\end{array}$




\section{Details}

sm.pathclus uses movMF (Section 3.7.1) to cluster pierce points on a unit hemisphere below each station. Pierce points are calculated for straight line station-event paths intersect the unit hemisphere. This function automatically divides the stations up so it does not have to be run individually.

\section{Output}

Vectors of the cuspids for each cluster are saved in path along with 3D figure of the clustered pierce points, a simple 2D map of the clusters and the ros diagrams of each cluster. The name of cuspid text files contain the station and the p-value returned from a test (i.e. WPRZ_cluster5_p-val_0.196)

\section{Examples}

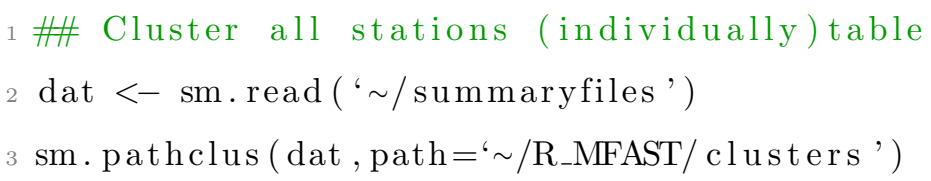

\section{E.2.5 sm.plot}

\section{Description}

Rose plots of of axial data (e.g. shear wave splitting polarisations)

\section{Usage}

sm. $\operatorname{proj}($ data, name $1=$ "raw.eps", name $2=$ "double.eps", path $=$ " $\sim$ ", cols=" blue", antipo$\mathrm{dal}=$ "lightblue", bins $=16, \mathrm{kd}=\mathrm{TRUE}$, arrow $=\mathrm{TRUE}$, medarrow $=F A L S E$, sym $=16$ ) 


\section{Arguments}

$\begin{array}{ll}\text { data } & \text { Vector of axial values (polarisations) } \\ \text { name1 } & \text { Name of the output file } \\ \text { name2 } & \text { Name of the output file (double angled plot) } \\ \text { path } & \text { Location to save output } \\ \text { cols } & \text { Colour of points } \\ \text { antipodal } & \text { Colour of antipodal points } \\ \text { bins } & \text { Number of bins for rose plot } \\ \text { kd } & \text { Option to plot kernel density estimate (with a smoothing bandwidth of } 10 \text { ) } \\ \text { arrow } & \text { Option to plot average arrow } \\ \text { medarrow } & \text { Option to plot median arrow } \\ \text { sym } & \text { Symbols for plotting individual measurements. Set to } 27 \text { for no symbols }\end{array}$

\section{Details}

sm.plot creates rose plots for visualisation of of axial data

\section{Examples}

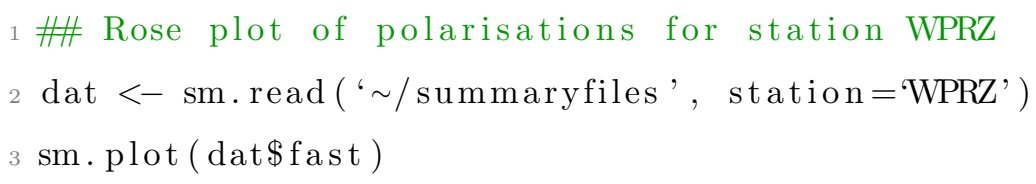

\section{E.2.6 sm.proj}

\section{Description}

Projection map of shear wave splitting measurements 


\section{Usage}

sm. $\operatorname{proj}($ summf, $\operatorname{lm}=2$, pierce=1.5, savpath=“ $\sim$ ", savnam="proj.png”, mlat=NULL, mlon=NULL, zoom $=13, x v e c=N U L L, y v e c=N U L L$, hvec $=$ NULL)

\section{Arguments}

$\begin{array}{ll}\text { summf } & \text {.summ dataframe (output of sm.read) } \\ \text { Im } & \text { Length multiplier } \\ \text { saerce } & \text { Pierce depth of projection (km from sea level) } \\ \text { savnam } & \text { Location to save output map } \\ \text { mlat } & \text { Name of output map } \\ \text { mlon } & \text { Latitude to centre map on } \\ \text { zoom } & \text { Zoom of getgooglemap }() \text { function (see ggmap documentation) } \\ \text { xvec } & \text { Vector longitude of perturbations to adjust station name locations }\left(^{\circ} \text { ) }\right. \\ \text { yvec } & \text { Vector latitude perturbations to station name locations }\left(^{\circ}\right) \\ \text { hvec } & \text { Vector of station elevations (in order of unique(summf } \$ \text { stat }) \text { ) }\end{array}$

\section{Details}

sm.proj projects shear wave splitting measurements onto a map. The point where the station-event path pierces the given depth (pierce) is projected to the surface and a vector is drawn. The vector is oriented in the direction of the fast polarisation with its length scaled with delay time (multiplied by $\mathrm{Im}$ ).

\section{Examples}

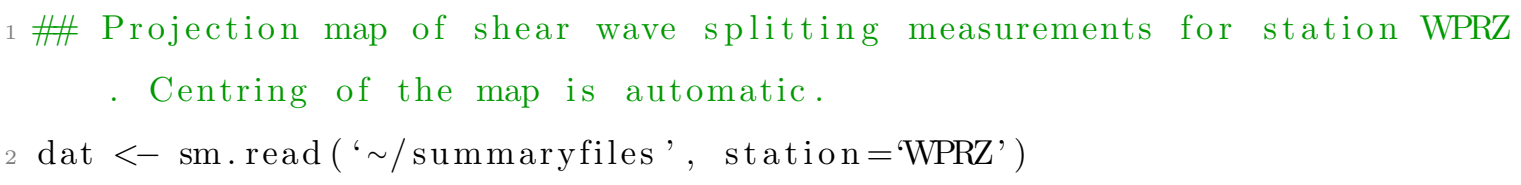




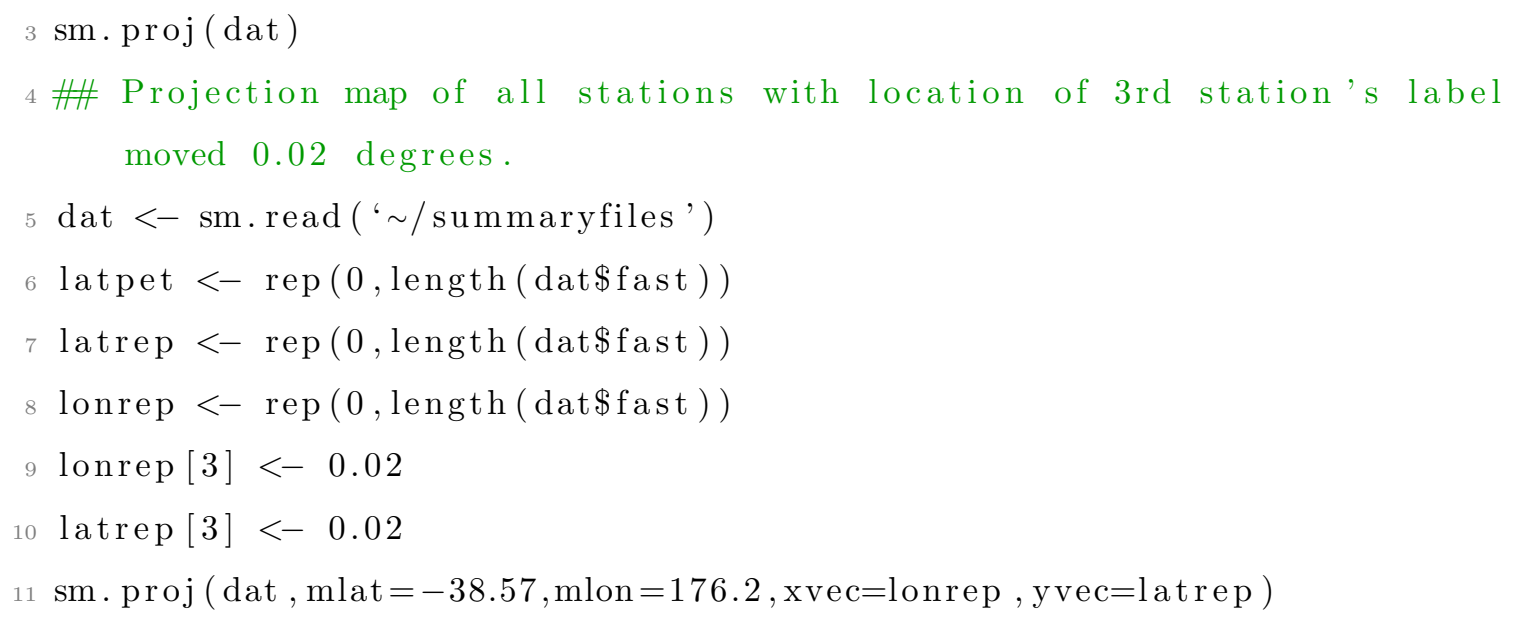

\section{E.2.7 sm.read}

\section{Description}

Reads and grades a raw .summ file

\section{Usage}

sm. $\operatorname{read}($ path, tlagmax $=1$, station $=N U L L, \operatorname{minl}=0, \operatorname{minsnr}=3$, type $=2)$

sm.read_l(path, tlagmax $=0.4$, station $=N U L L, \operatorname{minl}=0, \operatorname{minsnr}=3$, type $=2$ )

sm.read_vl (path, tlagmax $=0.2$, station $=N U L L, \operatorname{minl}=0, \operatorname{minsnr}=3$,type $=2)$

\section{Arguments}

$\begin{array}{ll}\text { path } & \text { Path to folder containing .summ files } \\ \text { tlagmax } & \text { Maximum time delay } \\ \text { station } & \text { Station(s) to read in. NULL reads all stations } \\ \text { minl } & \text { Minimum value of lambdamax allowed } \\ \text { minsnr } & \text { Minimum signal to noise ratio allowed } \\ \text { type } & \text { Grading method (see details) }\end{array}$




\section{Details}

sm.read and its variants read in raw .summ files (ungraded), grades the measurements and then returns a dataframe of the $\mathrm{AB}$ grade measurements (depending on the grading process). Grading is either type $=1$ where the $\mathrm{AB}$ measurement with the best filter from a unique event are chosen or the default type $=2$ where the measurement with the lowest error from an earthquake is chosen (detailed in Castellazzi et al. 2015). type $=2$ has an additional column finalgrade where measurements are assigned F1 (where only one of the three best filters for a unique event is AB grade), F2 (where two are $\mathrm{AB}$ and are within $10^{\circ}$ of each other), and F3 (where three are high grade and within $10^{\circ}$ of each other). A third category, F2b, indicates that there are three high grade measurements but only two are within $10^{\circ}$ of each other. sm.read_l and sm.read_vl are for the local and very local versions of the MFAST code respectively.

\section{Format}

The output of sm.read is a dataframe containing all the columns of a summ file. Column names match those in the .summ files except they do not contain any symbols or numbers except for underscores (i.e. "10dist_(ev-stat)" would "dist_evstat"). Column 1 "event" records the filter number used. All values remain identical except fast (fast polarisation) which is converted to radians and given the data type "circular".

\section{Examples}

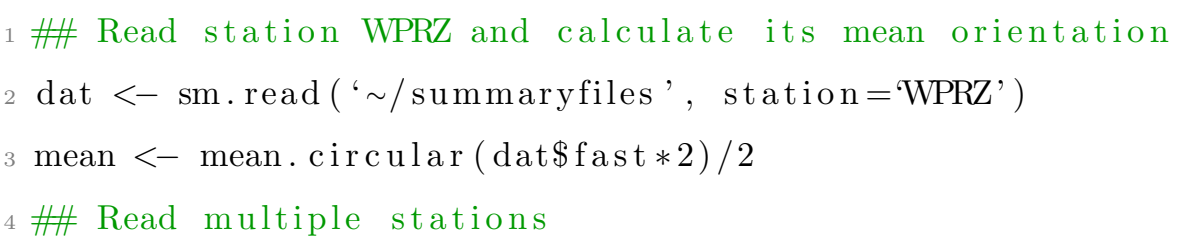


5 dat $<-$ sm.read (' $/$ summary files', station=c('WPRZ', 'ARAZ'))

\section{Notes}

IMPORTANT: There is a bug in MFAST whereby, if you have periods in your event names, an incorrect cuspid (unique event identifier) is saved. This prevents sm.read from working. To fix this navigate to your mfast/bin folder and add cuspid $=\$\{\mathrm{fn} \%$.* $*$.fb* $\}$; cuspid $=\$\{$ cuspid $\% .0\}$ to line 154 in mfast_logfiles (as of MFAST v2.2). This should work for all cases unless your names end in ".0".

\section{E.2.8 sm.readraw}

\section{Description}

Reads and returns .summ file

\section{Usage}

sm.readraw $($ path, tlagmax $=1$, station $=$ NULL, $\operatorname{minsnr}=3$ )

\section{Arguments}

$\begin{array}{ll}\text { path } & \text { Path to folder containing .summ files } \\ \text { tlagmax } & \text { Maximum time delay } \\ \text { station } & \text { Station(s) to read in. NULL reads all stations } \\ \text { minsnr } & \text { Minimum signal to noise ratio allowed }\end{array}$

\section{Details}

sm.readraw read in raw .summ files (ungraded), grades the measurements and then returns a dataframe of the all the measurements in the summ file (including all three filters for each event). 


\section{Format}

See sm.read (Section E.2.7).

\section{Examples}

1 \#\# Read station WPRZ

2 dat $<-$ sm.readraw (' $/$ summaryfiles', station $=$ 'WPRZ')

3 \#\# Read multiple stations

4 dat $<-$ sm. readraw (' $/$ summary files', station=c('WPRZ', 'ARAZ'))

\section{Notes}

See sm.read (Section E.2.7).

\section{E.2.9 sm.stde}

\section{Description}

Calculates standard error of a mean orientation using a bootstrap method

\section{Usage}

sm.stde $($ data, seed $=$ NULL, iter $=9999)$

\section{Arguments}

data Vector of fast polarisations (radians, undoubled)

seed Random number generator state. Set to allow reproducibility

iter Number of iterations for bootstrapping 


\section{Details}

Standard error is a measure of how close the sample mean is to the mean of the underlying population of fast polarisations. sm.stde calculates the standard error by re-sampling (with replacement) the doubled fast polarisations and calculating the mean of the re-sampled values. This is repeated iter times with the mean calculated each time. The standard error is the standard deviation of these means. Angles are doubled and then halved within the function.

\section{Examples}

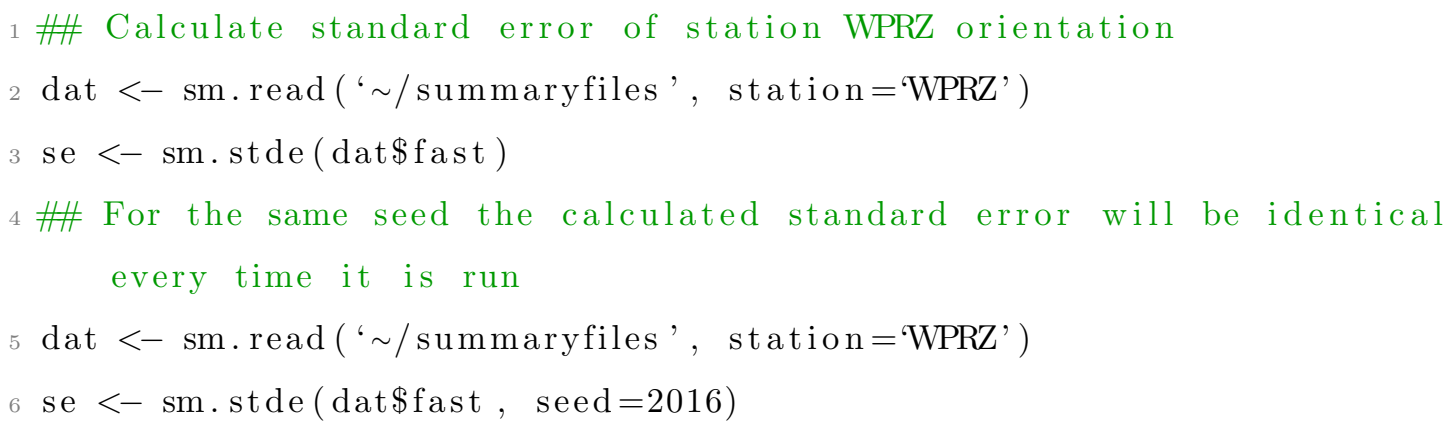




\section{Appendix F}

\section{NZGW conference paper}

The following paper (Mroczek et al. 2016) was presented in proceedings at the New Zealand Geothermal Workshop 2016. It represents preliminary results and processing that we later include and refine in this thesis. 


\title{
SHEAR WAVE SPLITTING AT ROTOKAWA AND NGATAMARIKI GEOTHERMAL FIELDS FOR 2015
}

\author{
Stefan Mroczek ${ }^{1}$, Martha Savage ${ }^{1}$, Chet Hopp ${ }^{1}$ and Steven Sewell ${ }^{1,2}$ \\ ${ }^{1}$ Victoria University of Wellington, PO Box 600, Wellington 6140, New Zealand \\ ${ }^{2}$ Mercury, PO Box 245, Rotorua 3040, New Zealand \\ stefan.mroczek@vuw.ac.nz
}

Keywords: Rotokawa, Ngatamariki, shear wave splitting, seismic anisotropy, fractures.

\begin{abstract}
In order to investigate the fracture orientation in the Ngatamariki and Rotokawa geothermal fields and compare them to the orientation of maximum horizontal stress, we determine the shear wave splitting for 1919 earthquakes across 22 stations during 2015.
\end{abstract}

The seismic catalogue provides a large number of microearthquakes from both fields. Due to the volume of unique events (4670), shear wave phases are picked automatically. We carry out automatic shear wave splitting measurements using the Multiple Filter Automatic Splitting Technique (MFAST). The shear wave splitting measurements are interpreted in the context of the stress in the region of Rotokawa and Ngatamariki by applying principles from circular statistics.

Preliminary results indicate that the mean fast azimuth measured from most stations in Ngatamariki agree with the expected NE-SW direction of maximum horizontal stress for the region. Stations located in Rotokawa show some tendency towards mean fast azimuths in a N-S direction, however they are generally more variable, perhaps indicating a more complex relationship between maximum horizontal stress and shear wave splitting measurements within the field.

\section{INTRODUCTION}

Seismic anisotropy is a material property in which a seismic wave's velocity is dependent on the direction it is travelling and on the polarisation of the wave. Shear wave splitting is a method of determining seismic anisotropy and when applied in fractured (geothermal) reservoirs it has the potential to provide a useful tool for measuring the orientation and density of fractures within the system (Elkibbi, Yang, and Rial 2005). Knowledge of the orientation and density of fractures within fields is in turn potentially useful for optimising field operations (e.g. through inclusion in numerical reservoir models) and for targeting production and injection wells.

The predominant cause of seismic anisotropy in the crust (measurable by shear wave splitting) is from fluid filled fractures which are often aligned with the maximum horizontal stress direction (Crampin and Peacock 2008). By measuring the shear wave splitting, the orientation and density of these fractures can be inferred. For an actively exploited geothermal field knowledge of these parameters could help to inform operations. Temporal variations could also indicate changes in the fractures and stress state within the reservoirs caused by field operations (e.g. increased fracturing due to stimulation around injection wells).
The geothermal fields of both Ngatamariki and Rotokawa have extensive seismic monitoring. They are also the subject of other geological and geophysical studies thus allowing comparison with other measures of stress and structure. Both fields are located within the Taupo Volcanic Zone (TVZ). The two fields' proximity to each other allows earthquakes from both fields to be studied together.

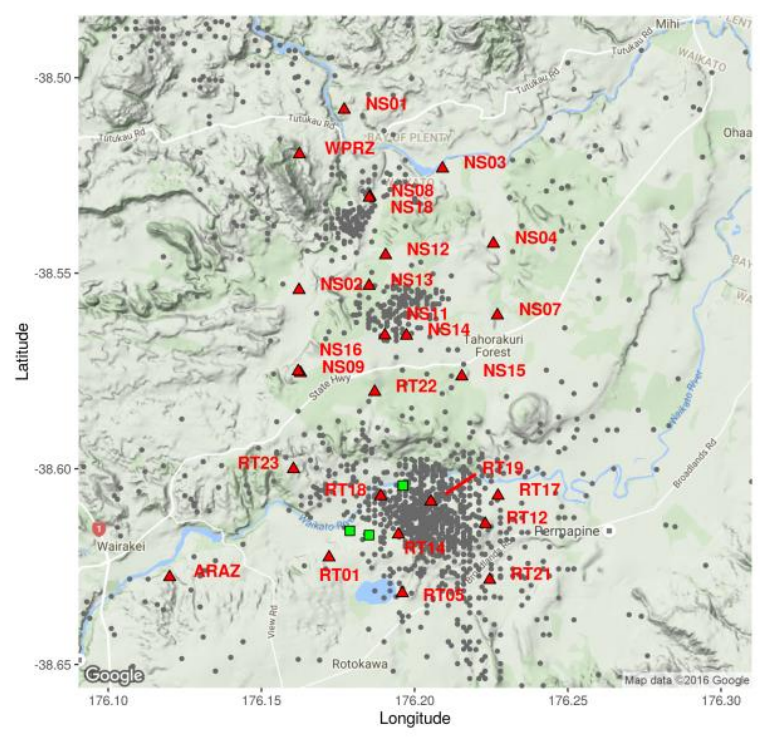

Figure 1: Events with at least one high grade shear wave splitting measurement for 2015 with station locations marked in red. Northern clusters are located in Ngatamariki while the southern cluster is located in Rotokawa. Green squares show wellhead locations for wells studied by Massiot et al. (2015) and McNamara, Massiot, et al. (2015).

Micro-earthquake events used for this shear wave splitting analysis are those from the 2015 catalogue from the region encompassing both fields, enhanced with 2466 additional detections using the matched-filter technique, made by Hopp et al. (this volume). The detections included are those which are detected on at least nine stations and which have a correlation of greater than 0.5 with their template. Automatic S-wave picking, using spickerC of Castellazzi et al. (2015) (Section 2.1), allows approximately $23 \%$ of events detected on a single station to have their shear wave phase determined. Due to the volume of events picked we have been able to employ statistical methods and tests including clustering and comparison of means, all of which require a sufficiently large numbers of measurements. In total 1919 unique events were used (Figure 1).

Sherburn et al. (2013) consider most of the seismicity at Rotokawa to be induced by deep reinjection of condensate 
and brine. It is likely that the seismicity at Ngatamariki is induced by similar mechanisms whereby the pre-fractured rock is contracted by cool reinjected fluids allowing fractures to reactivate (Sherburn et al. 2013).

\subsection{Shear wave splitting}

Shear wave splitting occurs when a shear wave enters an anisotropic medium and is split because the component polarised in one particular direction travels faster than the orthogonal component (Savage 1999). Shear wave splitting is characterised by two variables: fast azimuth (polarisation), $\varphi$, controlled by the anisotropic symmetry system and its orientation, and delay time, $\delta$ t, between the arrival of the fast and orthogonal polarisations (Savage 1999). The fast azimuth is an axial quantity so has a $180^{\circ}$ ambiguity (a fast azimuth of $0^{\circ}$ is the same as one of $180^{\circ}$ ). In many statistical operations an axial quantity can be conveniently treated as a simple direction by doubling the angle value, performing the operation and then halving the result.

The prevalent cause of shear wave splitting in the upper crust is likely to be fluid filled fractures that align with the local orientation of maximum horizontal stress $\left(\mathrm{S}_{\mathrm{Hmax}}\right)$ (Crampin and Peacock 2008). Fast azimuths are controlled by both the anisotropic medium and the propagation direction, but for many systems the fast directions are similar for a wide range of propagation angles (e.g. Babuska and Cara (1991)). Fractures not aligned with $S_{H \max }$ tend to be closed and their fluid forced into vertical fractures aligned with $S_{\text {Hmax. }}$ Thus the fast azimuth aligns with the local direction of $S_{H \max }$ and the delay time gives an indication of the fracture density along the station-event path.

Various studies have observed that, when close to faults, measured fast azimuths align with the fault strike (e.g. Evans et al. (1995), Zhang and Schwartz (1994), Zinke and Zoback (2000)). This alignment may be due to fractures or rock fabric resulting from fault parallel shear (Evans et al. 1995). The TVZ is a normal faulting regime so $\mathrm{S}_{\mathrm{H} \max }$ is expected to be parallel to the strike of the faults. Shear wave splitting resulting from either fractures or faults may be indistinguishable from each other in special cases when the faults align with $\mathrm{S}_{\mathrm{H} \max }$ (e.g. in a normal faulting regime).

Measures of $\mathrm{S}_{\mathrm{Hmax}}$ regionally from focal mechanisms (Townend et al. 2012) and more locally from borehole features from three boreholes in Rotokawa (McNamara, Massiot, et al. 2015) provide a comparison for fast azimuth measurements. Fracture distributions from cores and televiewer logs in Rotokawa (Massiot et al. 2015) also provide direct information on the orientation and density of fractures that may be causing the anisotropy being measured. The large-scale geological structure of the Rotokawa and Ngatamariki reservoirs is also well known (McNamara, Sewell, et al. 2016, Chambefort et al. 2016).

In this paper we report measurements of shear wave splitting in Ngatamariki and Rotokawa and use cluster analysis of station-event paths to interpret the results in terms of fracture and stress orientation.

\section{METHODS}

\subsection{Automatic S-picking}

Shear wave splitting measurements require the shear wave phase to be determined. The volume of events in the catalogue make manual picking time prohibitive so instead an automatic phase picker is used, spickerC of Castellazzi et al. (2015).
spickerC uses a combination of three different detection and picking methods: short term average versus long term average ratio (STA/LTA) as described by Allen (1978), polarisation detection based on the approach of Cichowicz (1993) and autoregressive picking using the Akaike Information Criterion (AR-AIC) as described by Leonard and Kennett (1999). The final pick is a weighted mean of the three methods with a final grade based on the error estimate and the signal to noise ratio around the pick (Castellazzi et al. 2015).

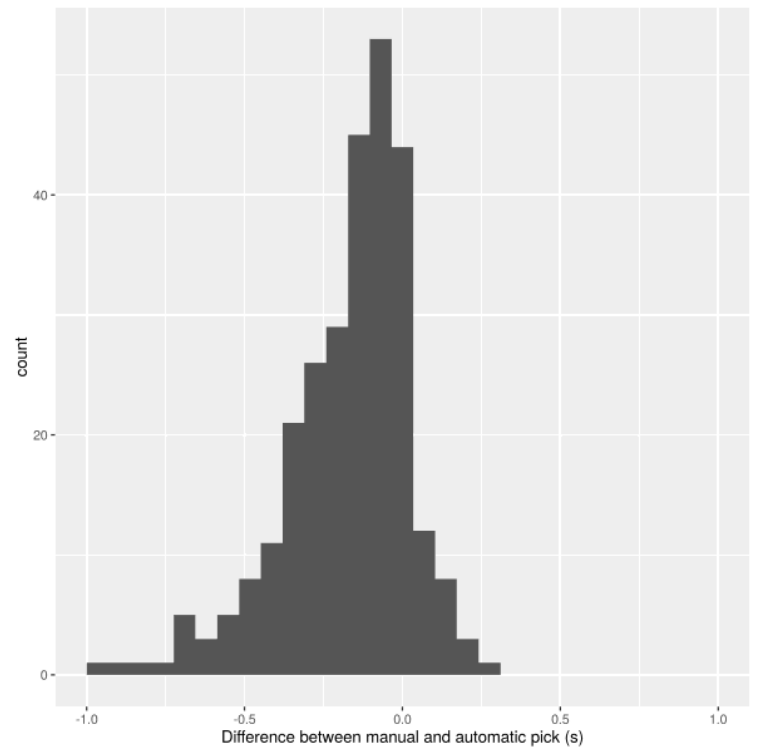

Figure 2: Time difference between earliest AR-AIC pick and manual pick for all stations in Rotokawa during 2013.

The automatic shear wave picking program (S-picker) was originally written by Diehl et al. (2009). The version used here was modified from version 1.4 .0 for use with local earthquakes in New Zealand by Castellazzi et al. (2015). Spicker was used by Castellazzi et al. (2015) in conjunction with MFAST (shear wave splitting program) to make automatic picking and splitting measurements at Ruapehu Volcano in New Zealand. Corrections and modifications were made to S-picker (updating to version 1.4.0.a) by Castellazzi et al. (2015) and are described therein. This most recent version of S-picker (1.4.0.a), used in processing here, is known as spickerC.

spickerC categorises its automatic picks into classes depending on their assigned error value. The class 0 category is the category with the lowest error. In this study class0 picks have an error of $\leq 0.3$ seconds and a signal to noise ratio (SNR) of $\geq 3$. Picks in the class0 category are those which are then processed in MFAST. Following the method of Castellazzi et al. (2015), we use the earliest AR-AIC pick from the class 0 category. This better approximates manual picks as well as being what is expected by MFAST (Castellazzi et al. 2015).

\subsection{1 spickerC parameters}

spickerC was trained using one hundred manually picked events detected in 2013 from station RT01, located in Rotokawa (Greenbank 2014). The parameters used in spickerC were chosen in a trial and error process. Initial values were set similarly to those of Castellazzi et al. (2015) with some modifications based on the change in setting from volcanic to geothermal. The parameters were then varied 
individually. After a parameter was changed spickerC was rerun. An increase in the number of class0 picks was considered a positive change and was kept, while no change or a decrease in class0 picks was reverted. Those parameters which changed from Castellazzi et al. (2015) are shown in Table 1.

Most changes to parameters resulted in a change of less than 10 in the number of class 0 picks. Increasing the band of allowable $\mathrm{Vp} / \mathrm{Vs}$ (P-wave to $\mathrm{S}$-wave velocity ratio), from that of Castellazzi et al. (2015), almost doubled the number of class0 picks made from 40 to 74 .

Before picking, data was filtered with both a high pass $(0.5$ $\mathrm{Hz}$ ) and a Wood-Anderson filter. The Wood-Anderson filter integrates to displacement and acts like a low pass filter (Nabelek, Braunmiller, and Phillips 2013). The Vp/Vs band was set to between 1.2 and 2.0 (mean of 1.6).

Figure 2 shows the difference between the earliest AR-AIC automatic pick and the manual pick from events in Rotokawa from March to June 2013 (Greenbank 2014) with eight outliers greater than one second removed. Most picks lie within half a second of each other however the distribution is skewed to the left with a mean of -0.17 seconds (manual picks are generally made at later times than the earliest corresponding AR-AIC pick). This is likely due to manual picks not always selecting the earliest arrival of the split shear wave.

Table 1: spickerC parameters changed from Castellazzi et al. (2015) (Ruapehu data). See the Supporting information of Castellazzi et al. (2015) for parameters and their descriptions.

\begin{tabular}{|l|r|r|}
\hline Parameter & Old Val. & New Val. \\
\hline \hline sta $(\mathrm{sec})$ & 0.2 & 0.15 \\
\hline lta $(\mathrm{sec})$ & 2 & 1.5 \\
\hline tup $(\mathrm{sec})$ & 0.2 & 0.1 \\
\hline tdw $(\mathrm{sec})$ & 0.1 & 0.05 \\
\hline Noise model window $(\mathrm{sec})$ & 1.2 & 1.1 \\
\hline Signal model window $(\mathrm{sec})$ & 1.2 & 1.1 \\
\hline Order noise model & 10 & 15 \\
\hline Order signal model & 10 & 16 \\
\hline
\end{tabular}

\subsection{Automatic shear wave splitting (MFAST)}

Shear wave splitting measurements are made using the Multiple Filter Automatic Splitting Technique (MFAST) of Savage et al. (2010a), Wessel (2010). Here we briefly summarise the method contained therein.

MFAST uses a combination of the Silver and Chan (1991) splitting method (with corrections from Walsh, Arnold, and Savage (2013)) and cluster analysis of splitting measurements (Teanby, Kendall, and Van der Baan 2004). The waveforms are bandpass filtered and the three best filters are selected based on the product of signal to noise ratio and the bandwidth of the filter. The splitting measurements are made on each of these best filters. Measurements are made by running the splitting algorithm on a set of windows each with different splitting parameters. The best windows are those whose parameters best remove the splitting. The best window is selected using the cluster analysis of Teanby, Kendall, and Van der Baan (2004). The measurements are graded based on the standard deviations of the clusters and how consistent the clusters are with each other (Savage et al. 2010a).

Measurements are graded null if fast azimuth is within 20 degrees of the incoming $\mathrm{S}$ polarisation. In this study, measurements with delay time less than $80 \%$ of the maximum time delay ( 0.2 seconds), signal to noise ratio greater than three, error in fast azimuth of less than 20 degrees and with an A or B cluster grade were kept for further analysis and are henceforth referred to as high grade measurements. If events have high grade measurements from more than one of their three best filters the one from the best filter is chosen.

MFAST requires an S pick (for a located earthquake) which in this case we made using spickerC (see previous section).

\subsubsection{MFAST parameters}

The most common bandpass filter selected in processing was $1-30 \mathrm{~Hz}$. Mean delay time for the (high grade) data is 0.087 seconds. The main MFAST parameters are listed in Table 2. Refer to the MFAST manual (Wessel, Savage, and Teanby 2016) for a description of these parameters. The "very local" variation of the MFAST codes is used in processing.

Table 2: MFAST processing parameters. See Wessel, Savage, and Teanby (2016).

\begin{tabular}{|l|r|}
\hline Parameter & Value \\
\hline \hline t_win_snr & $3 \mathrm{~s}$ \\
\hline t_err & $0.05 \mathrm{~s}$ \\
\hline SNRmax & 3 \\
\hline tlagmax & $0.2 \mathrm{~s}$ \\
\hline dtlagmax & $0.05 \mathrm{~s}$ \\
\hline twin_freq & $3 \mathrm{~s}$ \\
\hline
\end{tabular}

\subsection{Earthquake path clustering}

Clustering events by their location is useful as it provides an indication of where measured anisotropy originates. When investigating temporal changes it also allows migration of the seismicity to be ruled out as a cause of changing anisotropy.

Seismicity is generally confined to a single area in Rotokawa and two distinct areas in Ngatamariki. Clustering these events purely by location proved inadequate for three reasons. Firstly, to be able to recover statistically significant means the clusters had to contain a sufficient number of measurements. Secondly, doing a simple clustering with kmeans gave clusters which contained all events from a particular field, thus the assumption that events in a cluster have a similar location no longer holds. Finally, given that shear wave splitting is measured not at a point but rather along the station-event path, it is more intuitive to cluster the station-event paths so that events with similar paths to the same station group together.

To approximate this clustering we calculate the pierce points of the station-event paths on a unit sphere below each station (Figure 3). A pierce point is the location where a path intersects a surface. Only stations with more than 55 measurements are clustered using this method as sufficiently 
large samples are required. Mixtures of von Mises-Fisher distributions (the spherical equivalent of a normal distribution) are then fit to the pierce points using the movMF package (Hornik and Grün 2014) in the R software environment (R Core Team 2013). This clusters the pierce points by location on the sphere (each distribution is fit to a set of pierce points which form a cluster).

Data for each station is clustered separately. The clustering (movMF) algorithm (Hornik and Grün 2014) requires that the number of clusters be set before running. The clustering is repeated with one to seven clusters being fit to the pierce points. The best number of clusters is chosen using the Bayesian Information Criterion. This is a comparative measure of how well a model fits the observations with a penalty for adding more clusters to prevent over fitting. The number of clusters which minimises the criterion for a particular station is chosen and the corresponding clustering is kept.

To determine if the fast azimuths of events within a specific cluster depart from circular uniformity (i.e. there is a dominant fast azimuth) the Rayleigh test is used. The Rayleigh test is a statistical test for circular uniformity. If the concentration of data points around a circle is above a certain threshold then the hypothesis that there is circular uniformity (no dominant mean direction) is rejected (Pewsey, Neuhäuser, and Ruxton 2013). This test is carried out on the (doubled) fast azimuths from the events in each of the clusters determined by movMF. If the p-value from the Rayleigh test is less than 0.1 the test is considered a pass and the cluster is determined to have a statistically significant mean fast azimuth. The p-value indicates the significance of the mean direction where 0.1 indicates that there is a small amount of evidence for a single mean fast azimuth while a $\mathrm{p}$-value of 0.01 indicates strong evidence for a single mean fast azimuth.

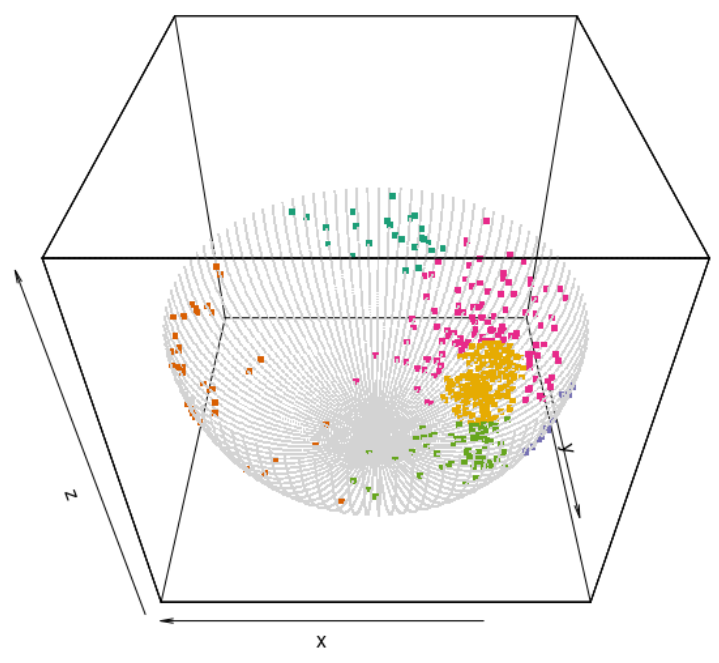

Figure 3: Pierce points on sphere beneath station RT01. Colours represent different path clusters. The yellow cluster gives a statistically significant fast azimuth originating from events in Rotokawa.

For stations with more than one cluster of paths which pass the Rayleigh test the mean fast azimuths of these clusters were compared using the bootstrap version of Watson's Nonparametric test following the method of Fisher (1993) (Pewsey, Neuhäuser, and Ruxton 2013). If there is less than medium evidence ( $p$-value $>0.05$ ) that the means from each cluster are different they are grouped together. The test is non-parametric so allows us to test for equality of means without needing to specify the distributions of the data within the clusters. Bootstrapping allows clusters with smaller sample sizes to be included.

Clusters with a wide range of station-event paths generally fail the Rayleigh test, suggesting such measurements from clusters should be inspected on an event by event basis and that spatial variation in anisotropy is present.

At angles of incidence greater than $35^{\circ}$ (where vertical incidence is $0^{\circ}$ ) converted phases can interfere with shear wave splitting measurements at the surface (Nuttli 1961, Booth and Crampin 1985). Measurements with angles of incidence below $35^{\circ}$ fall into the shear wave window where measurements are free of this interference. Figure 3 is calculated using straight line paths (i.e. uniform velocity model) and, due to low velocity layers near the surface, many studies consider events below $45^{\circ}$ to be in the shear wave window (e.g. Volti and Crampin 2003). However, this is too restrictive and so removes too many useful events (Savage et al. 2010b). This is especially true in geothermal areas which are characterised by low velocities. For example, $64 \%$ of events with angles of incidence greater than $45^{\circ}$ on Figure 3 are in fact within the shear wave window when angles of incidence are calculated using a more accurate velocity model. If we restrict the measurements to include only those within $35^{\circ}$ incidence using the Rotokawa velocity model of Rawlinson (2011), the average fast directions are significantly different from the full dataset at only one station (RT05) using Watson's nonparametric test (described earlier in this section). Thus, for this study, we choose not to remove measurements based on their angle of incidence.

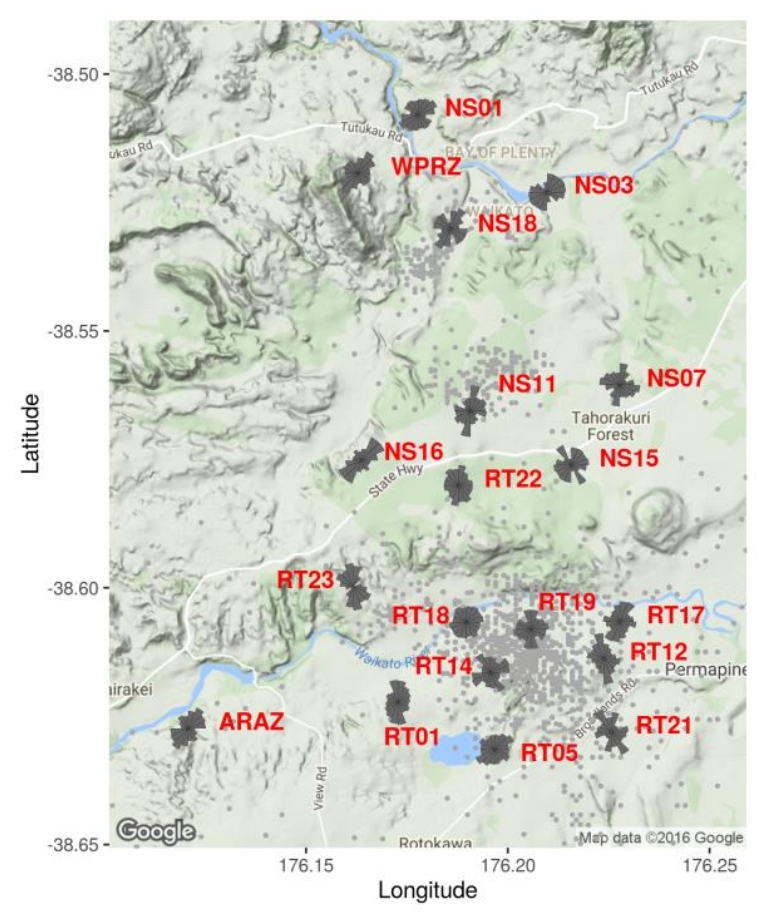

Figure 4: Fast azimuth rose plots.

\section{RESULTS}

\subsection{Station Results}

The Rayleigh test (described in Section 2.3) is used to divide the stations into two categories: those with a single dominant fast azimuth for all measurements (which pass the Rayleigh 
test with a p-value less than 0.05) and those with uniformly distributed fast azimuths (which fail). These are described in Section 3.1.1 and 3.1.2 respectively.

Rose plots of fast azimuths measured at each station are shown in Figure 4. Stations NS04 and NSO2 are excluded, both having less than three measurements. NS12-NS14 are borehole stations and do not have their orientation calibrated, so all measurements of fast azimuth will have an unknown fixed offset. They are also not reported here. During the study period two stations were disbanded and moved slightly. To account for this we merge the measurements post processing. NS09 and NS16 are merged (labelled NS16) and NS08 and NS18 are merged (labelled NS18). There is no significant evidence that the mean fast azimuth of each station differs after being moved (using Watson's Nonparametric test as described in Section 2.3).

Across all stations, $23 \%$ of events (for each station) were successfully picked using spickerC. $34 \%$ of these picked events gave high grade shear wave splitting measurements. Thus, roughly $8 \%$ of catalogue events (for an individual station) result in high grade shear wave splitting measurement. The rate of successful S-wave picks is lower than the $28 \%$ of Castellazzi et al. (2015) which is due to the addition of matched-filter detections to our catalogue (there $27 \%$ successful pick rate when excluding matched filter detections). The rate of high grade shear wave splitting measurements from our picks is significantly lower, 34\% versus $60 \%$ of of Castellazzi et al. (2015) (Ruapehu data), perhaps in part due to difference in the maximum delay time used in MFAST processing. Our study area being much smaller, we only consider events with a time delay of below 0.16 seconds to be high grade, versus 0.6 seconds in Castellazzi et al. (2015). Also, Castellazzi et al. (2015) only includes events of magnitude 1.5 and above while in this study we have no minimum magnitude.

Table 3: Stations with a dominant fast azimuth. Columns are: mean fast azimuth (degrees from north), standard error, number of events, number of events with s-wave picked and number of high grade shear wave splitting measurements.

\begin{tabular}{|l|r|r|r|r|r|}
\hline Station & F.A. $\left(^{\circ}\right)$ & S.E. $\left(^{\circ}\right)$ & Events & Picks & Me. \\
\hline \hline ARAZ & 34 & 8.7 & 1771 & 266 & 39 \\
\hline WPRZ & 43 & 3.3 & 3752 & 534 & 346 \\
\hline NS01 & 37 & 7.0 & 2963 & 999 & 253 \\
\hline NS03 & 57 & 4.6 & 1700 & 288 & 474 \\
\hline NS11 & 31 & 11 & 1636 & 165 & 46 \\
\hline NS16 & 49 & 2.6 & 2285 & 480 & 148 \\
\hline RT01 & 9 & 2.4 & 3824 & 1415 & 489 \\
\hline RT05 & 51 & 5.0 & 3233 & 1835 & 555 \\
\hline RT12 & -19 & 3.8 & 2742 & 909 & 253 \\
\hline RT17 & 26 & 5.6 & 3021 & 742 & 250 \\
\hline RT23 & -19 & 7.2 & 1141 & 126 & 33 \\
\hline
\end{tabular}

\subsubsection{Dominant fast azimuth}

The mean fast azimuth for Ngatamariki (NS \& WPRZ stations) along with ARAZ fall into the band of $31^{\circ}$ to $57^{\circ}$ (Table 3). The mean orientation of $S_{H \max }$ in the TVZ, measured from focal mechanisms (Townend et al. 2012), is

NE-SW $\left(39.38^{\circ}\right.$ to $50.62^{\circ}$ by standard definition) or ENEWSW $\left(61.88^{\circ}\right.$ to $\left.73.12^{\circ}\right)$. The mean fast azimuth of all these stations are within one standard error of these $\mathrm{S}_{\mathrm{Hmax}}$ orientations. In situ-borehole measurements of $S_{H \max }$ orientation made in Rotokawa by McNamara, Massiot, et al. (2015) also agree with the observed fast azimuths for these stations.

Stations in Rotokawa (RT) have mean fast azimuths distributed around a $\mathrm{N}-\mathrm{S}$ orientation ranging from $-19^{\circ}$ to $26^{\circ}$ with RT05 as an outlier with an mean of $51^{\circ}$.

Standard errors, quoted for each station in Table 3, provide a measure of how close the sample mean is to the mean of the underlying population of fast azimuths. Here we calculate the standard error by re-sampling (with replacement) the doubled fast azimuths and calculating the mean of the resampled values. Re-sampling is where values are drawn randomly from a sample, in this case the sample is the fast azimuths from a single station. This is repeated 9999 times with the mean calculated each time. The standard error is the standard deviation of these means. The standard error is halved to give the standard error of the axial mean fast azimuths (see Section 1.1).

Table 4: Stations with uniformly distributed fast azimuths. Columns are: number of events, number of events with s-wave picked and number of high grade shear wave splitting measurements.

\begin{tabular}{|l|r|r|r|}
\hline \multicolumn{1}{|c|}{ Station } & \multicolumn{1}{c|}{ Events } & \multicolumn{1}{c|}{ Picks } & \multicolumn{1}{c|}{ Measurements } \\
\hline \hline NS07 & 1547 & 131 & 39 \\
\hline NS15 & 1119 & 185 & 52 \\
\hline NS18 & 1364 & 163 & 49 \\
\hline RT14 & 2879 & 355 & 73 \\
\hline RT18 & 3258 & 1067 & 274 \\
\hline RT19 & 3209 & 259 & 26 \\
\hline RT21 & 2783 & 123 & 36 \\
\hline RT22 & 524 & 119 & 29 \\
\hline
\end{tabular}

Table 5: Earthquake path clusters. Columns are: mean fast azimuth (degrees from north), standard error and number of events with splitting measurement.

\begin{tabular}{|l|r|r|r|}
\hline \multicolumn{1}{|c|}{ Station } & F.A. $\left(^{\circ}\right)$ & S.E. $\left({ }^{\circ}\right)$ & Measurements \\
\hline \hline WPRZ & 41 & 3.0 & 222 \\
\hline NS01 & 44 & 13.7 & 50 \\
\hline NS03 & 65 & 4.5 & 61 \\
\hline NS16 & 41 & 12.2 & 74 \\
\hline RT01 (N) & 11 & 4.3 & 113 \\
\hline RT01 (S) & 25 & 9.5 & 63 \\
\hline RT01 (Fig6) & 5 & 2.5 & 238 \\
\hline RT05 (Fig5) & -6 & 8.7 & 46 \\
\hline RT05 (Fig6) & 44 & 9.1 & 190 \\
\hline RT12 & -17 & 3.2 & 193 \\
\hline RT17 & 31 & 6.3 & 114 \\
\hline
\end{tabular}




\subsubsection{Uniformly distributed fast azimuths}

Mean fast azimuths are not quoted for stations which fail the Rayleigh test as they are not statistically significant. Table 4 shows a summary of these stations. Ngatamariki stations (NS) without a dominant fast azimuth generally all have a small number of measurements. It is likely that, with additional measurements, a dominant azimuth would become apparent. Rotokawa stations (RT) with a large number of measurements and uniformly distributed fast azimuths are generally located much closer to seismicity than other stations which pass the test (see Figure 1).

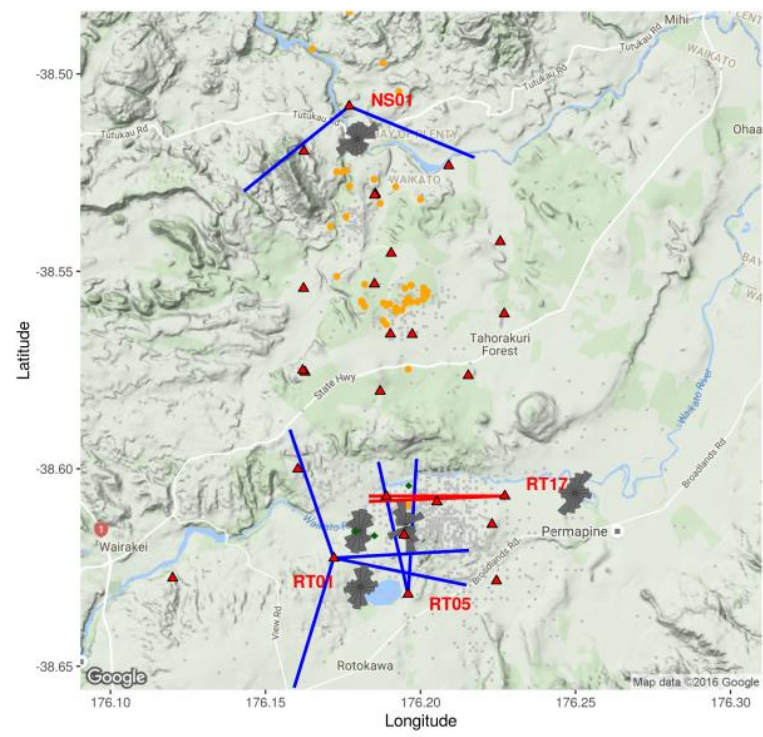

Figure 5: Fast azimuth rose plots from station-event path clusters outside of red cones and within blue cones. Events making up RT05's N-S cluster are highlighted in orange.

\subsection{Path clustering}

Figure 5 and 6 show the result of the path clustering method in Section 2.3. Means are summarised in Table 5. All measurements for each rose diagram have station-event paths which lie within the cones formed by blue lines or outside for those with red lines. This gives an indication of where the measured anisotropy is originating.

Stations RT01, RT12 and RT17 all show that their N-S character originates from the main Rotokawa cluster. RT17 and RT12 only have very narrow bands of paths which have been excluded. RT01 also has two smaller path clusters which originate outside of the main Rotokawa cluster which also have N-S mean fast azimuths.

Station RT05 has one cluster with a strong N-S component $\left(-6^{\circ}\right)$ typical of Rotokawa stations (orange in Figure 5). However, the earthquakes making up this cluster originate largely from southern Ngatamariki rather than from the main body of Rotokawa events.

Most of the anisotropy observed in Ngatamariki originates along the path from Rotokawa.

\section{DISCUSSION}

Station RT18 has a large number of high grade shear wave splitting measurements associated with it yet fails the Rayleigh test and thus do not appear to have any dominant fast azimuth. Stations RT14 and RT19 also fail although these two stations do have a smaller number of measurements. All three of these stations are located much closer to the main group of Rotokawa earthquakes, which produce their splitting measurements, than stations with dominant azimuths. Stations located slightly further away (e.g RT01, RT12, RT17) tend to pass the Rayleigh test and have a roughly $\mathrm{N}-\mathrm{S}$ orientation. Ngatamariki stations with a larger number of events $(>60)$ all pass the Rayleigh test and all have mean fast azimuths closer to the expected NE-SW trend of stress in the region. RT05 appears to be in-between these two extremes where it passes the Rayleigh test but has a low concentration around its mean fast azimuth. Without the addition of matched-filter detections to the catalogue RT05 fails the Rayleigh test while still having a large amount of measurements (282).

It is possible that whatever is causing stations close to seismicity to have uniformly distributed fast azimuths is also related to the anomalous $\mathrm{N}-\mathrm{S}$ trend. However, one of the clusters from RT05 has a strong N-S character while the events in the cluster are located in Ngatamariki (highlighted in orange on Figure 6). This suggests that the N-S character is local to Rotokawa and not a side effect of the observed uniformly distributed fast azimuths. Along with NE-SW and

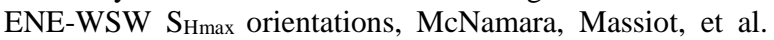
(2015) also measured NNE-SSW which match with RT17. $\mathrm{N}-\mathrm{S}$ orientated cracks are also common in all three boreholes. Massiot et al. (2015) also identified a family of fractures in Rotokawa boreholes with N-S orientations perhaps indicating a relationship with the N-S fast azimuths observed in Rotokawa. Wellhead locations are plotted in green on Figure 1 (RK32, RK30L1 and RK18L2).

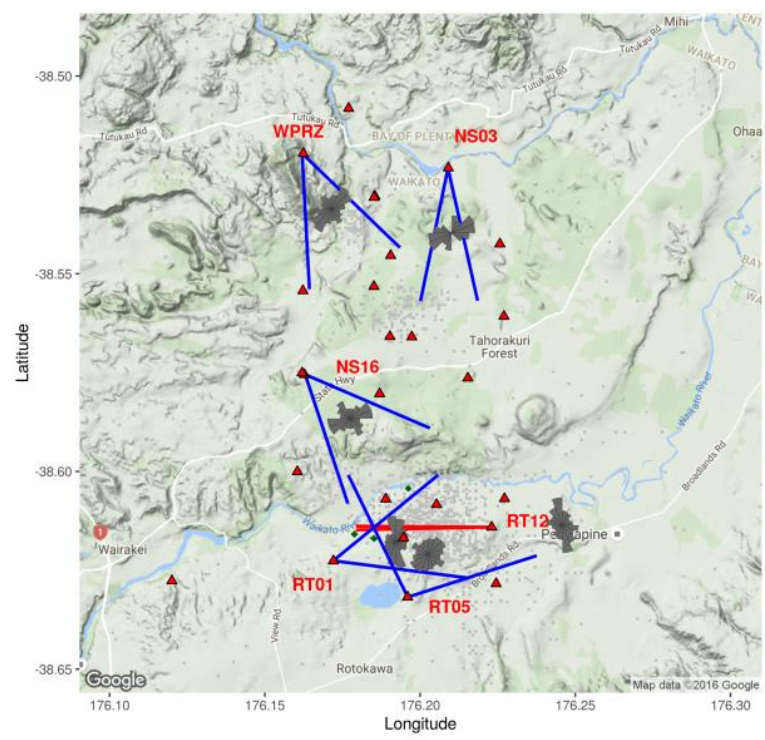

Figure 6: Fast azimuth rose plots from station-event path clusters outside of red cones and within blue cones.

\section{CONCLUSIONS}

The orientation of mean fast azimuths determined in the study for Rotokawa and Ngatamariki is $28^{\circ}$ to $57^{\circ}$ with rotation to a broader range of orientations centred $\mathrm{N}-\mathrm{S}$ within the Rotokawa field.

Of stations with a dominant fast azimuth, those in Ngatamariki and station ARAZ (all located outside of Rotokawa) have mean fast azimuths which coincide with the expected values of NE-SW to ENE-WSW from in-situ and 
regional measurements (McNamara, Massiot, et al. 2015, Townend et al. 2012). The mean fast azimuths of stations located in Rotokawa fell into a broader range of $-21^{\circ}$ to $38^{\circ}$ centred on an N-S orientation.

Clustering of station-event paths revealed that the NE-SW character of stations outside Rotokawa largely originated from events within Rotokawa. While Rotokawa stations measured N-S anisotropy from the seismicity within the field, station RT05 showed some events originating in Ngatamariki which also have a N-S orientation. This suggests that the $\mathrm{N}-\mathrm{S}$ mean fast azimuths measured in Rotokawa are local to the field and not a result of proximity to the seismicity. N-S fractures are common in all three boreholes located in Rotokawa measured by McNamara, Massiot, et al. (2015).

\section{FUTURE WORK}

Further investigation into why stations located close to seismicity may be failing the Rayleigh test is required.

Preliminary tests indicate no statistically significant change in time for most path clusters. The addition of more years to the catalogue will allow temporal changes to be investigated. This would also allow the measurements to be broken into time periods expected to show differences (e.g. after significant stimulation). Also, stations which failed the Rayleigh test tended to only have a small number of measurements so an increase in events would be beneficial.

Three borehole stations (NS12 to NS14) are not orientated. Work to correctly orientate these stations will add a significant amount of high quality measurements in the centre of the Ngatamariki field as well as providing a comparison between borehole and surface stations.

The Rayleigh test only tests the significance of a single mean fast azimuth. Thus stations which potentially have more than one mean fast azimuth (i.e sampling more than one population of anisotropy) could fail. Investigation into stations which potentially have more than one mean fast azimuth could give more information about the origin of anisotropy in Rotokawa.

In addition, shear wave splitting tomography, similar to that of Johnson, Savage, and Townend (2011) could be used to identify regions of differing anisotropy and to investigate temporal changes.

\section{ACKNOWLEDGEMENTS}

We would like to thank Mercury and the Rotokawa Joint Venture (Mercury and Tauhara No.2 Trust) for their support of this research in awarding an MSc scholarship to Stefan Mroczek, providing access to data and permission to publish this work. We are also grateful to GeoNet for providing the earthquake data.

Figures were made using the ggplot2 (Wickham 2009) and ggmap (Kahle and Wickham 2013) packages. Statistical analysis was carried out using the circular package of Agostinelli and Lund (2013). All packages are within the R software environment (R Core Team 2013). The Seismic Analysis Code was used in the processing of seismic data (Helffrich, Wookey, and Bastow 2013).

\section{REFERENCES}

Agostinelli, C. and Lund, U.: R package circular: Circular Statistics (version 0.4-7). CA: Department of Environmental Sciences, Informatics and Statistics, Ca' Foscari University, Venice, Italy. UL: Department of Statistics, California Polytechnic State University, San Luis Obispo, California, USA, 2013.

Allen, R.V.: Automatic earthquake recognition and timing from single traces. Bulletin of the Seismological Society of America 68.5 (1978).

Babuska, V. and Cara, M.: Seismic anisotropy in the Earth. Vol. 10. Springer Science \& Business Media, 1991.

Booth, D.C. and Crampin, S.: Shear-wave polarizations on a curved wavefront at an isotropic free surface. Geophysical Journal International 83.1 (1985).

Castellazzi, C., et al.: Shear wave automatic picking and splitting measurements at Ruapehu volcano, New Zealand. Journal of Geophysical Research. Solid Earth 120.5 (2015).

Chambefort, I., et al.: Ngatamariki Geothermal Field, New Zealand: Geology, geophysics, chemistry and conceptual model. Geothermics 59 (2016).

Cichowicz, A.: An automatic S-phase picker. Bulletin of the Seismological Society of America 83.1 (1993).

Crampin, S. and Peacock, S.: A review of the current understanding of seismic shear-wave splitting in the Earth's crust and common fallacies in interpretation. Wave Motion 45.6 (2008).

Diehl, T., et al.: Automatic S-wave picker for local earthquake tomography. Bulletin of the Seismological Society of America 99.3 (2009).

Elkibbi, M., Yang, M. and Rial, J.A.: Crack-induced anisotropy models in The Geysers geothermal field. Geophysical Journal International 162.3 (2005).

Evans, J.R., et al.: Shear-wave splitting from local earthquakes at The Geysers geothermal field, California. Geophysical Research Letters 22.4 (1995).

Fisher, N.I.: Statistical analysis of circular data. (1993).

Greenbank, E. Seismic anisotropy and fractures at Rotokawa Geothermal Field. Unpublished manuscript.

Helffrich, G., Wookey, J. and Bastow, I.: The Seismic Analysis Code: A Primer and User's Guide. Cambridge University Press, 2013.

Hopp, C., et al.: Matched filter earthquake detection and double difference relocation at Rotokawa and Ngatamariki geothermal areas: January to November, 2015. This volume.

Hornik, K. and Grün, B.: movMF: An R Package for Fitting Mixtures of von Mises-Fisher Distributions. Journal of Statistical Software 58.10 (2014). 
Johnson, J.H., Savage, M.K. and Townend, J.: Distinguishing between stress-induced and structural anisotropy at Mount Ruapehu Volcano, New Zealand. Journal of Geophysical Research: Solid Earth 116.B12 (2011).

Kahle, D. and Wickham, H.: ggmap: Spatial Visualization with ggplot2. The R Journal 5.1 (2013).

Leonard, M. and Kennett, B.L.N.: Multi-component autoregressive techniques for the analysis of seismograms. Physics of the Earth and Planetary Interiors 113.1 (1999).

Massiot, C., et al.: Fracture Width and Spacing Distributions from Borehole Televiewer Logs and Cores in the Rotokawa Geothermal Field, New Zealand. Proceedings World Geothermal Congress 2015. (2015)

McNamara, D.D., Massiot, C., et al.: Heterogeneity of structure and stress in the Rotokawa Geothermal Field, New Zealand. Journal of Geophysical Research: Solid Earth 120.2 (2015).

McNamara, D.D., Sewell, S., et al.: A review of the Rotokawa Geothermal Field, New Zealand. Geothermics 59 (2016).

Nabelek, J.L., Braunmiller, J. and Phillips, W.S.: Source and path calibration in regions of poor crustal propagation using temporary, large-aperture, high-resolution seismic arrays. Tech. rep. 2013.

Nuttli, O.: The effect of the Earth's surface on the $\mathrm{S}$ wave particle motion. Bulletin of the Seismological Society of America 51.2 (1961)

Rawlinson, Z.: Microseismicity associated with actively exploited geothermal systems: earthquake detection and probabilistic location at Rotokawa and statistical seismic network design at Kawerau. MSc thesis. Victoria University of Wellington, 2011.

$\mathrm{R}$ Core Team. R: A Language and Environment for Statistical Computing. Vienna, Austria: R Foundation for Statistical Computing, 2013.

Savage, M.K.: Seismic anisotropy and mantle deformation: What have we learned from shear wave splitting? Reviews of Geophysics 37.1 (1999).

Savage, M.K., et al.: Automatic measurement of shear wave splitting and applications to time varying anisotropy at Mount Ruapehu volcano, New Zealand. Journal of Geophysical Research, Solid Earth 115.12 (2010a).

Savage, M.K., et al.: Stress magnitude and its temporal variation at Mt. Asama Volcano, Japan, from seismic anisotropy and GPS. Earth and Planetary Science Letters 290.3 (2010b).

Sherburn, S., et al.: Microseismicity at Rotokawa geothermal field, 2008 to 2012. Proceedings of the 35th New Zealand geothermal workshop. Rotorua, New Zealand. (2013).

Silver, P.G. and Chan, W.W.: Shear wave splitting and subcontinental mantle deformation. Journal of Geophysical Research: Solid Earth 96.B10 (1991).
Teanby, N.A., Kendall, J.M. and Van der Baan, M Automation of shear-wave splitting measurements using cluster analysis. Bulletin of the Seismological Society of America 94.2 (2004).

Townend, J., et al.: Three-dimensional variations in presentday tectonic stress along the Australia-Pacific plate boundary in New Zealand. Earth and Planetary Science Letters 353 (2012).

Volti, T. and Crampin, S.: A four-year study of shear-wave splitting in Iceland: 1. Background and preliminary analysis. Geological Society, London, Special Publications 212.1 (2003).

Walsh, E., Arnold, R. and Savage, M.K.: Silver and Chan revisited. Journal of Geophysical Research. Solid Earth 118.10 (2013).

Wessel, A.: Shear Wave Splitting Measurements at Mt. Ruapehu Volcano, New Zealand. MSc thesis. Victoria University of Wellington, 2010.

Wessel, A., Savage, M.K. and Teanby, N.A.: Manual for the Multiple Filter Automatic Splitting Technique (MFAST) processing codes, Version 2.1. Victoria University of Wellington, 2016.

Wickham, H.: ggplot2: Elegant Graphics for Data Analysis. Springer-Verlag New York, 2009.

Zhang, Z. and Schwartz, S.Y.: Seismic anisotropy in the shallow crust of the Loma Prieta segment of the San Andreas fault system. Journal of Geophysical Research: Solid Earth 99.B5 (1994).

Zinke, J.C. and Zoback, M.D.: Structure-related and stressinduced shear-wave velocity anisotropy: observations from microearthquakes near the Calaveras Fault in Central California. Bulletin of the Seismological Society of America 90.5 (2000). 
